Fall 2016

\title{
A Measurement of the Parity-Violating Asymmetry in Aluminum and its Contribution to A Measurement of the Proton's Weak Charge
}

Joshua Allen Magee

College of William and Mary, jmagee@email.wm.edu

Follow this and additional works at: https://scholarworks.wm.edu/etd

Part of the Physics Commons

\section{Recommended Citation}

Magee, Joshua Allen, "A Measurement of the Parity-Violating Asymmetry in Aluminum and its Contribution to A Measurement of the Proton's Weak Charge" (2016). Dissertations, Theses, and Masters Projects. Paper 1477068118.

http://doi.org/10.21220/S22012

This Dissertation is brought to you for free and open access by the Theses, Dissertations, \& Master Projects at W\&M ScholarWorks. It has been accepted for inclusion in Dissertations, Theses, and Masters Projects by an authorized administrator of W\&M ScholarWorks. For more information, please contact scholarworks@wm.edu. 
A Measurement of the Parity-Violating Asymmetry in Aluminum and its Contribution to a Measurement of the Proton's Weak Charge

\author{
Joshua Allen Magee \\ Bridgewater, MA
}

MS Physics, College of William and Mary, 2010

BS Mechanical Engineering, University of Hartford, 2007

A Dissertation presented to the Graduate Faculty of the College of William and Mary in Candidacy for the Degree of Doctor of Philosophy

Department of Physics The College of William and Mary

May 2016 
(C) 2016

Joshua Allen Magee

All Rights Reserved 
APPROVAL PAGE

This dissertation is submitted in partial fulfillment of the requirements for the degree of

Doctor of Philosophy

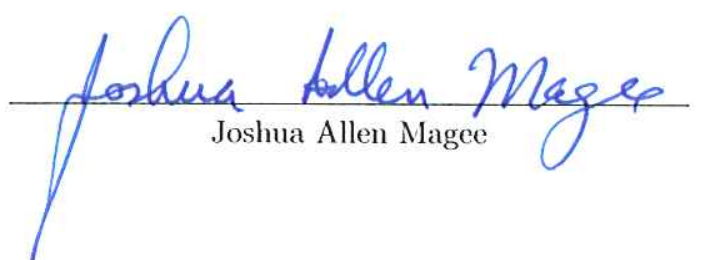

Approved by the Committee February 2016

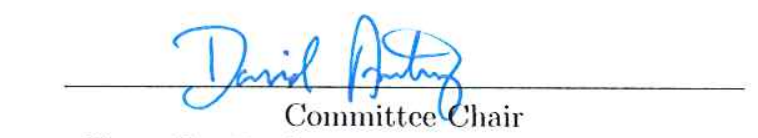

Chancellor Professor David Armstrong, Physics The College of William and May

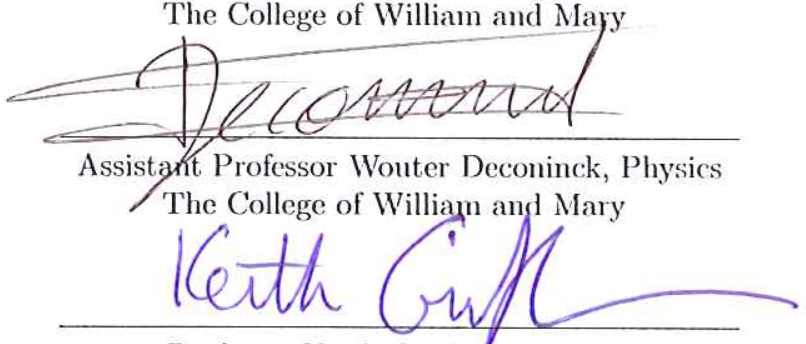

Professor Keith Griffioon, Physics The College of William and Mary

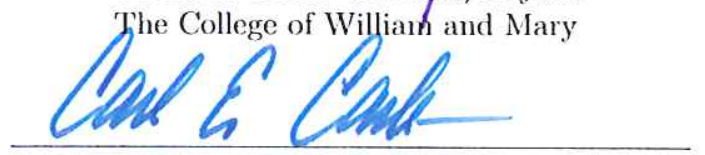

Professor Carl Carlson, Physics The College of William and Mary

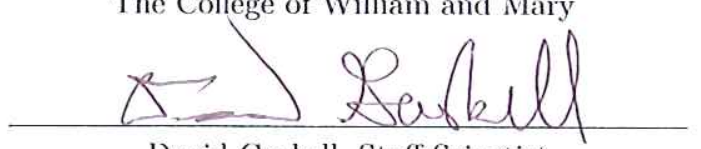

David Gaskell, Staff Scientist. Thomas Jefferson National Accelerator Facility 


\begin{abstract}
The $Q_{\text {weak }}$ experiment, which ran at the Thomas Jefferson National Accelerator Facility, made a precision measurement of the proton's weak charge, $\mathrm{Q}_{W}^{p}$. The weak charge is extracted via a measurement of the parity-violating asymmetry in elastic electron-proton scattering from hydrogen at low momentum transfer $\left(Q^{2}=0.025 \mathrm{GeV}^{2}\right)$. This result is directly related to the electroweak mixing angle, $\sin ^{2}\left(\theta_{W}\right)$, a fundamental parameter in the Standard Model of particle physics. This provides a precision test sensitive to new, as yet unknown, fundamental physics.

This dissertation focuses on two central corrections to the $Q_{\text {weak }}$ measurement: the target window contribution and sub-percent determination of the electron beam polarization. The aluminum target windows contribute approximately $30 \%$ of the measured asymmetry. Removal of this background requires precise measurements of both the elastic electron-aluminum scattering rate and its parity-violating asymmetry. The results reported here are the most precise measurement of the $Q_{\text {weak }}$ target dilution and asymmetry to date. The parity-violating asymmetry for the aluminum alloy was found to be $1.6174 \pm$ 0.0704 (stat.) \pm 0.0113 (sys.) parts-per-million. The first sub-percent precision polarization measurements made from the Hall C Møller polarimeter are also reported, with systematic uncertainties of $0.84 \%$.
\end{abstract}




\section{Table of Contents}

Table of Contents

Acknowledgements $\quad$ vi

Dedication $\quad$ viii

List of Tables $\quad$ ix

List of Figures $\quad$ xi

1 The Electroweak Standard Model 1

1.1 Introduction to the Standard Model . . . . . . . . . . . . . . . 2

1.2 Symmetries . . . . . . . . . . . . . . . . . 4

1.2.1 Discrete symmetries . . . . . . . . . . . . . . 5

1.3 Electroweak unification $\ldots \ldots \ldots \ldots \ldots \ldots$

1.3.1 Electroweak gauge structure . . . . . . . . . . . . . 7

1.3.2 Spontaneous symmetry breaking and the Higgs mechanism . . . 9

1.3.3 Neutral current interactions . . . . . . . . . . . . . . . . . 11

1.3.4 Nucleon weak charges . . . . . . . . . . . . . . . . . . 12

1.4 Electroweak radiative corrections . . . . . . . . . . . . . . . 13

2 Parity-Violating Electron Scattering and the $Q_{\text {weak }}$ Experiment 18

2.1 Electromagnetic form factors $\ldots \ldots \ldots \ldots \ldots$

2.1 .1 Neutral weak form factors . . . . . . . . . . . . . . 20

2.2 Cross sections for $e+p$ and $e+A l$ scattering $\ldots \ldots 22$

2.3 Parity-violating asymmetries and electron scattering . . . . . . . . . 23 


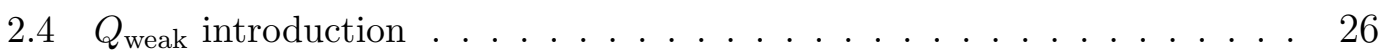

3 Experimental Setup 32

3.1 Experimental apparatus . . . . . . . . . . . . . . . 33

3.2 Beam delivery . . . . . . . . . . . . . . . . . . . . . 34

3.2 .1 Photocathode and beam generation $\ldots \ldots \ldots \ldots$

3.2 .2 Injector and slow helicity reversals $\ldots \ldots \ldots$

3.2 .3 Accelerator . . . . . . . . . . . . . . . . . 39

3.3 Beam diagnostics . . . . . . . . . . . . . . . . . . . . . . . 39

3.3 .1 Beam position monitors . . . . . . . . . . . . . . 40

3.3 .2 Beam current monitors . . . . . . . . . . . . . . . 41

3.3.3 Beam energy determination . . . . . . . . . . . . . . . 42

3.3 .4 Beam modulation . . . . . . . . . . . . . . . 43

3.4 Polarimetry . . . . . . . . . . . . . . . . . . 45

3.4 .1 Møller Polarimeter . . . . . . . . . . . . . . . . . . . . 45

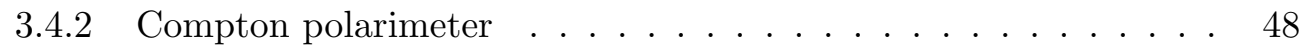

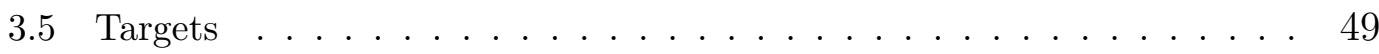

3.5.1 Primary LH2 target . . . . . . . . . . . . . . . . 49

3.5.2 Aluminum targets . . . . . . . . . . . . . . . . 52

3.6 Collimators . . . . . . . . . . . . . . . . . . 53

3.7 Spectrometer magnet . . . . . . . . . . . . . . . . 55

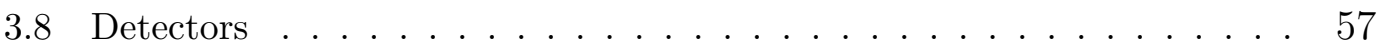

3.8 .1 Main detectors . . . . . . . . . . . . . . . 57

3.8.2 Focal plane scanner . . . . . . . . . . . . . . . . . . 59 
3.8 .3 Luminosity monitors . . . . . . . . . . . . . . . . . . . 60

3.9 Tracking system . . . . . . . . . . . . . . . . 61

3.9 .1 Horizontal drift chambers . . . . . . . . . . . . . . . 62

3.9.2 Vertical drift chambers . . . . . . . . . . . . . . . 62

3.9 .3 Trigger scintillators . . . . . . . . . . . . . . 63

3.10 Data acquisition . . . . . . . . . . . . . . 63

4 The Møller Polarimeter Analysis 65

4.1 Configuration during $Q_{\text {weak }} \ldots \ldots \ldots \ldots \ldots$. . . . . . . . . . . 65

4.2 Analyzing power determination and uncertainty . . . . . . . . . . . 69

4.2 .1 Nominal target polarization . . . . . . . . . . . . 70

4.2 .2 Target temperature . . . . . . . . . . . . 71

4.2 .3 Solenoid field uncertainty . . . . . . . . . . . . 72

4.2 .4 Quadrupole currents . . . . . . . . . . . . . . 73

4.2.5 Position and angle systematic uncertainty . . . . . . . . . 75

4.2 .6 Møller Run 1 optics effect . . . . . . . . . . . . . . . 78

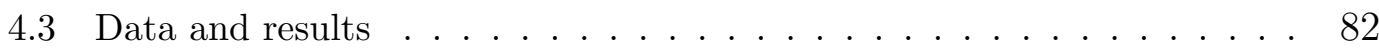

4.3 .1 Leakage corrections . . . . . . . . . . . . . . . . . 82

4.3 .2 Effect of the insertable half wave plate . . . . . . . . . 85

4.4 Møller summary . . . . . . . . . . . . . . . . . . . . . . 87

5 The Aluminum Window Contribution $\quad 89$

5.1 Aluminum overview . . . . . . . . . . . . . . . . . . 89

5.2 Dilution method . . . . . . . . . . . . . . . . . 92

5.2 .1 Measurement conditions . . . . . . . . . . . . . . 93 
5.2 .2 Reference target normalization . . . . . . . . . . . . . 103

5.3 Data and analysis........................... 105

5.4 Radiative corrections . . . . . . . . . . . . . . . 108

5.4.1 Simulation model uncertainties . . . . . . . . . . . . . . . . 110

5.5 Dilution final results . . . . . . . . . . . . . . . . . . . 112

6 The Parity-Violating Asymmetry of Aluminum 114

6.1 Aluminum asymmetry overview . . . . . . . . . . . . . . . . . 114

6.2 Data quality............................ 115

6.3 Asymmetry analysis . . . . . . . . . . . . . . . . . . 121

6.3.1 Raw asymmetry and background corrections . . . . . . . . . 123

6.3.2 Extracting the $Q_{\text {weak }}$ correction . . . . . . . . . . . 130

7 Concluding discussions $\quad 135$

7.1 Summary of findings . . . . . . . . . . . . . . 135

7.2 Current Run $2 Q_{\text {weak }}$ Results $\ldots \ldots \ldots \ldots$

7.3 Future work . . . . . . . . . . . . . . . . . . 141

$\begin{array}{ll}\text { Appendices } & 144\end{array}$

A Møller-Compton Cross Calibration 144

A.1 Systematics and method . . . . . . . . . . . . . . . . . . 144

A.2 Data, analysis, and results . . . . . . . . . . . 147

B Alternate Rate Determinations 151

B.1 Scaler based main detector deadtime determination . . . . . . . . . . . 151 
B.2 Event-mode technique . . . . . . . . . . . . . . . . 154

B.2.1 Rates in event-mode . . . . . . . . . . . . . . . . 155

B.2.2 Detector deadtimes . . . . . . . . . . . . . . . 157

B.2.3 Prescaling, DAQ deadtime, and time determination . . . . . 158

B.3 Data analysis . . . . . . . . . . . . . . . . . . . . 159

Bibliography 


\section{Acknowledgements}

This milestone was made possible in large-part due to the wonderful people surrounding me at William and Mary and Jefferson Lab. Without the generous gift of their time and help, this document would be, in some way, incomplete. More importantly, without them my life would be less colorful, rich, and fufilling. I will do my best to continue the grand tradition of naming everybody, but there is a near uncountable set, and I fear my attempts may be incomplete at points.

First I should thank everyone at William and Mary, in particular Marc Sher, who encouraged my application and acceptance into the program. As an engineer with little previous formal physics training, it was often overwhelming, but worth it. Everybody was so supportive, and I am grateful for the nuturing environment provided. Thank you to Paula, Carol, Elle, and Bonnie for putting up with my antics and crazy sense of humor, and for making my time there so much more enjoyable (and for always sharing the best sandwich in town).

I am indebted to my fellow $Q_{\text {weak }}$ graduate students. Many, many owl shifts (and late-night "shifts" at the bar) were spent with Manolis, Scott, Adesh, Wade, Anna, and Nur. Amrendra brought much needed joy and peace during many stressful times. The first $Q_{\text {weak }}$ graduate students to graduate, in particular John Leacock, Rakitha, and Buddhini, paved the way for the rest of us. Even though not a grad student, Jean-Francois deserves a shout-out for all of his cunning plans and willingness to share his wisdom. Everybody's hard work and clear descriptions made understanding this experiment fun and doable.

My fellow William and Mary students spent more time with me than anybody should have to. John Leckey taught me much about project management and that I had to concentrate on what's important in my analysis - not every technique needs refinement. Valerie was always willing to help in my analysis and with the farm. Jim Dowd always brought quick wit, lively debate, and thoughtful discussion. Rachel brought much joy to our meetings and became a dear friend. Josh Hoskins needs to be thanked for the constant trouble I caused him - he has an uncanny ability to put with with my bad jokes about being my namesake. Without his guidance and friendship at the beginning of my research, I never would have made it. Juan-Carlos was instrumental in developing my programming skills; a gift I appreciate daily. Thank you William and Mary, I am grateful for everything.

Team aluminum has my gratitude. Katherine taught me scientific honesty, and provided me the ultimate role model. Every day I sit down to work and hope my end result will make her happy; if it would I know it's solid and well thought out. I am so fortunate that Kurtis eventually joined our working group, as without his simulation expertise and drive, I would likely still be fighting with QwGEANT. I couldn't have made it without his abundant willingness to help. Rupesh provided many important behind the scenes discussions on the low-energy nuclear analysis of Chapter 6 , which has become increasingly important in my new position. 
I had many unofficial advisors from JLab, and I am grateful for the seemingly neverending patience they showed. All spent hundreds of hours going through the simplest and most mundane details to ensure that one day, I would finally figure it all out. Dave Mack, in particular, was a driving force early in the analysis, who taught me the importance of preparation and clear, cogent thought. Brad provided me all the coffee I stubbornly wouldn't drink, and was so fantastic at all things electronic. Likewise, Steve Wood provided an unbelievable amount of support, which made many difficult tasks doable. Roger Carlini provided a great sense of humor and also much wisdom to the experiment. Thank you also for your career guidance.

My William and Mary committee members provided numerous discussions throughout the years and much needed direction in this document's construction. Wouter was crucial throughout $Q_{\text {weak }}$ and provided many important clarifications. Keith and Carl did likewise. Additionally, they both provided much guidance and wisdom one fine evening in Pafos I'll never forget.

Special thanks to Victoria, for being my best Williamsburg friend and starting the only club I'd ever be a member of. Ben and Eric, two of my favorite people who constantly make physics fun and exciting, even during the days when things just aren't working. To say Greg Smith provided the best and most memorable discussions during the last five years, physics or otherwise, is an understatement. Thank you for all the boat rides! Much thanks to Rob Mahurin for cleverly providing the most important lessons I learned in graduate school, and providing additional wisdom every time we talk.

There is a final, very special thanks to Keyser Söze, to whom without his good graces I wouldn't be here, and the three Daves. Dave Hamlette, thank you for that day way back in May 2012 when you gave me an extra 30 minutes of beam time. Thanks to you, we were able to improve the agreement between aluminum data and simulation by a factor of two. Dave Gaskell and David Armstrong, you are my heroes. You both showed more grace and patience during some very trying times, especially as I was a fresh grad student, and I honestly hope someday to be half the scientists, educators, and gentlemen you are. Thank you for being such wonderful role models, and for believing in me fully, even when I couldn't believe in myself.

Lastly, I must thank my family. Thank you all for your patience and understanding; now that I'm finished we can all relax. And especially thank you for taking care of my two boys, Rosie and Tommy. I sure hope you two understand why I've disappeared, although I suspect you'll be happy to see me again, or maybe that will just be the catnip in my pocket... 
Dedicated to Rosencrantz and Tommy The two greatest boys a dad could hope for 


\section{List of Tables}

1.1 Neutral vector and axial-vector couplings in the Standard Model . . . . 12

1.2 Summary of electroweak radiative corrections $\ldots \ldots \ldots \ldots$

3.1 Typical operating parameters of $Q_{\text {weak }} \ldots \ldots \ldots \ldots$

3.2 Summary of regression schemes monitors . . . . . . . . . . . . . . . . 44

3.3 Aluminum dummy target thickness, areal density, measurement results . 54

4.1 Calculated temperature rise at Møller target center . . . . . . . . . . 72

4.2 Relative demagnetization of Møller target with temperature . . . . . . 72

4.3 Summary of Møller position/angle on target calculations . . . . . . . 78

4.4 Final Møller systematic uncertainty table for Run $2 \ldots$. . . . . . . 83

4.5 Final Møller systematic uncertainty table for Run 1 . . . . . . . . . . . 84

5.1 Double pulse resolutions for Run 1 and Run $2 \ldots \ldots$. . . . . . . 102

5.2 Reference target (Carbon) data by run . . . . . . . . . . . . . . . 104

5.3 Summary of average rates and correction sizes for the dilution . . . . . 106

5.4 Averaged raw dilution factors for Runs 1 and $2 \ldots \ldots$. . . . . . 108

5.5 Average dilution radiative correction factors . . . . . . . . . . . . . . 110

5.6 Final dilution values, including radiative corrections . . . . . . . . . 113

6.1 Raw aluminum asymmetry slug-averages and NULL. . . . . . . . . . . 124

6.2 Polarization values by slug . . . . . . . . . . . . . . . . 126

6.3 Corrected aluminum asymmetry slug-averages and NULL. . . . . . . . 127

6.4 Helicity correlated beam parameters during Run 2 aluminum running . 129

6.5 Average main detector sensitivity during Run2 aluminum running . . . 129

6.6 Regression effects on average aluminum assymetry $\ldots \ldots$. . . . . . 130 
6.7 Simulated upstream window physics process kinematics $\ldots \ldots$. . . . 133

6.8 Simulated downstream window physics process kinematics . . . . . . 133

6.9 Simulated solid aluminum target physics process kinematics . . . . . . . 134

7.1 Summary of final radiative corrections . . . . . . . . . . . . 137

7.2 Summary of final false asymmetries . . . . . . . . . . . . . . . 139

7.3 Summary of background asymmetries for $\mathrm{LH}_{2}$ running . . . . . . . . . 139

7.4 Summary of background dilutions for $\mathrm{LH}_{2}$ running . . . . . . . . . . 139

A.1 Møller uncertainties in the Møller-Compton cross-calibration . . . . . . 146

A.2 Compton uncertainty list f . . . . . . . . . . . . . 148

A.3 Møller-Compton cross calibration results . . . . . . . . . . . . . . 148

B.1 Scaler based deadtime constant $\tau$ s extracted . . . . . . . . . . . . 153 


\section{List of Figures}

1.1 The Standard Model Particle Content . . . . . . . . . . . . . . . . . . 3

1.2 Photon exchange Feynman diagram f . . . . . . . . . . . . . . 14

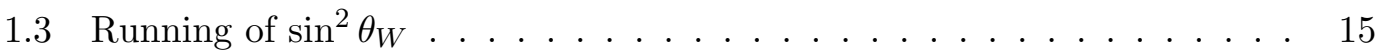

1.4 Relevent electroweak radiative corrections for $Q_{\text {weak }} \ldots \ldots \ldots$

$1.5 \square_{\gamma Z}$ energy dependence $\ldots \ldots \ldots \ldots \ldots \ldots \ldots$

2.1 Tree level $e p$ Feynman diagrams _ . . . . . . . . . . . . . . . . . . . 19

2.2 Net strangeness of the nucleon as a function of $Q^{2} \ldots \ldots 22$

2.3 Calculated $e+A l$ cross section compared to data . . . . . . . . . . 24

2.4 Global fit of parity-violating electron scattering data and extrapolation

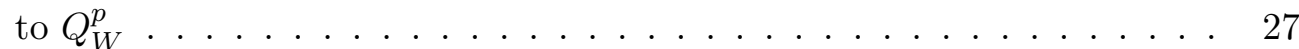

2.5 Constraints on the weak vector quark couplings $C_{1 u}$ and $C_{1 d} \ldots \ldots 28$

2.6 Experimental determination of $\sin ^{2} \theta_{W}$ evolution $\ldots \ldots . \ldots 29$

2.7 Comparison of sensitivity to new physics between $Q_{\text {weak }}$ and E158 . . 30

2.8 New physics mass reach probed by $Q_{\text {weak }} \ldots \ldots \ldots \ldots$

3.1 Kinematics of an elastic two-body process $\ldots \ldots \ldots 33$

3.2 The $Q_{\text {weak }}$ experimental apparatus . . . . . . . . . . . . . 34

3.3 Beam generation and delivery schematic $\ldots \ldots \ldots \ldots$

3.4 Laser spot on cathode and QE reduction . . . . . . . . . . . . . 36

3.5 Beam position monitor coordinate system . . . . . . . . . . . . . . 40

3.6 Møller analyzing power versus center-of-mass scattering angle . . . . . . 46

3.7 Schematic of the Hall C Møller polarimeter . . . . . . . . . . . . . . . 47

3.8 Schematic of the Hall C Compton polarimeter . . . . . . . . . . . . . . . 49

3.9 Primary $Q_{\text {weak }}$ target CFD design $\ldots \ldots \ldots \ldots$ 
3.10 Primary $Q_{\text {weak }}$ target schematic diagram . . . . . . . . . . . . 51

3.11 Effect of target boiling on the main detector asymmetry width $\ldots . .52$

3.12 Target ladder, solid target arrays $\ldots \ldots \ldots$. . . . . . . . . 53

3.13 Cross-section of experimental apparatus background sources . . . . . . 56

3.14 Main detector coordinate system . . . . . . . . . . . . . . 58

3.15 Photograph of main detector bars in-situ . . . . . . . . . . . . 59

3.16 Scattered electron profile at detector face . . . . . . . . . . 60

4.1 Schematic of the Hall C Møller polarimeter . . . . . . . . . . . . . . 66

4.2 Møller detector acceptance correlations ("tune plot") . . . . . . . . . 66

4.3 Photograph of large Møller quadrupole . . . . . . . . . . . . . . 67

4.4 Relative quadrupole field vs. time during Run 1 position scan . . . . . . 68

4.5 Møller analyzing power versus center-of-mass scattering angle with a broken optical transport system . . . . . . . . . . . . . 69

4.6 Magnetization dependence of pure iron for multiple foil tilt angles . . . 73

4.7 Relative rates as a function of Møller solenoid position . . . . . . . . . 74

4.8 Møller Q3 current systematic study . . . . . . . . . . . . . 75

4.9 Møller position sensitivity . . . . . . . . . . . . 76

4.10 Simulated polarization dependence on target $x$-position $\ldots \ldots 76$

4.11 Magneto-optical elements used to determine beam position on the Møller

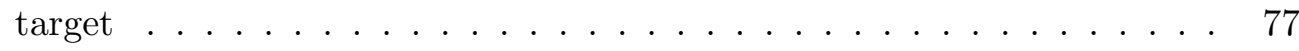

4.12 Procedure to calculate positions on the Møller target . . . . . . . . 78

4.13 POISSON Magnetic field maps for Møller quadrupole . . . . . . . . 79

4.14 Relative scattering rate in Møller during the position scan . . . . . . . 80

4.15 Plot of Møller polfac vs. day in Run $1 \ldots$. . . . . . . . . . . . 81

4.16 Effect of the inactive IHWP on polarization for Run $2 \ldots \ldots$. . . . 86

4.17 Effect of active IHWP on polarization for Run $2 \ldots \ldots$. . . . . 86

4.18 Polarization for each day by Wien flip vs. time . . . . . . . . . . 87 
4.19 Final $Q_{\text {weak }}$ Run 2 polarimetry results $\ldots \ldots \ldots 8$

4.20 Final $Q_{\text {weak }}$ Run 1 polarimetry results $\ldots \ldots \ldots$. . . . . . . 88

5.1 Elastic cross section and parity-violating asymmetry for $e \cdot p$ and $e \cdot A l$ scattering. . . . . . . . . . . . . . . . . . 91

5.2 Upstream target window scattering profile on main detector bar . . . . . 91

5.3 Tracking mode DAQ and trigger scintillator event-mode electronics schemat-

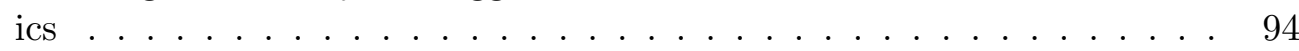

5.4 F1TDC trigger and hit selection diagram $\ldots \ldots \ldots . \ldots . \ldots 95$

5.5 Carbon reference target normalization with improper dead time correction 97

5.6 Trigger scintillator double pulse resolution . . . . . . . . . . . . . . . 98

5.7 Main detector double pulse resolution $\ldots \ldots \ldots$. . . . . . . . 98

5.8 Main detector pulse size versus second hit time . . . . . . . . . . . . 99

5.9 Main detector double pulse resolutions Run 1 . . . . . . . . . . . . 101

5.10 Main detector double pulse resolutions Run $2 \ldots \ldots 101$

5.11 Run 2 low-current beam current calibration . . . . . . . . . . . 103

5.12 Reference target normalization . . . . . . . . . . . . . 105

5.13 Run 1 empty and full hydrogen target rates . . . . . . . . . . . 105

5.14 Run 2 empty and full hydrogen target rates . . . . . . . . . . . . 106

5.15 Main detector rates normalized to MD1 . . . . . . . . . . 107

5.16 Raw aluminum dilution for Runs 1 and $2 \ldots \ldots$. . . . . . . 108

5.17 Rates from solid aluminum targets during magnetic field (QTOR) scans 111

5.18 Relative rates from solid aluminum targets during magnetic field (QTOR) scans . . . . . . . . . . . . . . . . . . . . 111

5.19 Relative rate difference between measured and simulated rates during a QTOR scan. . . . . . . . . . . . . . . . 112

5.20 Aluminum target window dilution with radiative corrections (Run 2) . . 113

6.1 Charge asymmetry minimization: acceptable transition run $\ldots \ldots 117$

6.2 Charge asymmetry minimization: unacceptable transition run . . . . . 117 
6.3 Charge asymmetry minimization: borderline transition run . . . . . 117

6.4 Aluminum charge asymmetry histogram . . . . . . . . . . . . . . 118

6.5 Wien 8 BCM78 double differences vs. runlet . . . . . . . . . . . . . . . 119

6.6 Wien 8 energy differences differences vs. runlet . . . . . . . . . . . 120

6.7 Main detector best fit line fit probabilities . . . . . . . . . . . . . . . 121

6.8 Raw aluminum asymmetry by slug . . . . . . . . . . . . . . . . . 123

6.9 Raw aluminum asymmetry by Wien . . . . . . . . . . . . . . . . 124

6.10 Regressed (set13) aluminum asymmetry by Wien . . . . . . . . . . 125

6.11 Beamline background correction sizes, by Wien . . . . . . . . . . 126

6.12 Corrected aluminum asymmetry by slug, (beamline background and po-

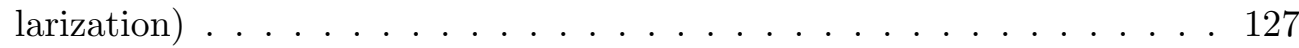

6.13 Corrected aluminum asymmetry by Wien (beamline background and po-

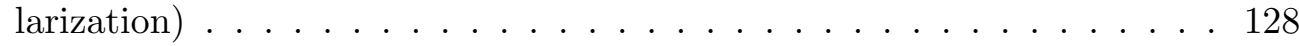

7.1 Asymmetry extrapolation to $Q_{W}^{p}$ (blinded result) . . . . . . . . . 140

7.2 Constraints on quark weak-vector couplings (blinded result) . . . . . . 141

7.3 Parity-violating asymmetry for elastic electron-aluminum scattering . 142

A.1 Møller current scan results . . . . . . . . . . . . . . . . . . . . . 145

A.2 Compton measurements at high/low currents . . . . . . . . . . 147

A.3 Møller-Compton cross-calibration results . . . . . . . . . . . . . . . . 149

A.4 Measured polarization versus current during Møller-Compton cross calibration . . . . . . . . . . . . . . . . 150

B.1 Evacuated target rate scan on an empty target . . . . . . . . . 153

B.2 Evacuated target rate scan $\tau$ extraction on an empty target . . . . . 153

B.3 Event-mode marriage simple example . . . . . . . . . . . . . 156

B.4 Software meantimes for trigger scintillators and main detectors . . . 156

B.5 Overlay of married and unmarried main detector spectra . . . . . . . . 157

B.6 Event-mode prescale study . . . . . . . . . . . . . . . . . . 159 
B.7 Reference target normalization with event-mode . . . . . . . . . 160 
Like all young men I set out to be a genius, but mercifully laughter intervened.

Clea Lawrence Durrell 


\section{Chapter 1}

\section{The Electroweak Standard Model}

ONE HUNDRED YEARS ago, Rutherford discovered that the atomic nucleus is a compact, positively-charged core $[1,2]$, thus heralding the modern era of nuclear and particle physics. Since then, a fundamental theory of interactions was developed, describing interactions in terms of three fundamental forces. This theory, called the Standard Model, has been enormously successful since its development in the 1960's and 1970's. Although much recent attention has focused on high-energy explorations at large laboratories, many modern scattering experiments are able to probe this fundamental structure and key predictions through low-energy, precision measurements of Standard Model quantities.

$Q_{\text {weak }}$ is one such experiment, focusing on the first experimental determination of the proton's weak charge, $Q_{W}^{p}$, through a measurement of the parity-violating asymmetry in elastic electron-proton scattering. This thesis discusses recent progress in the $Q_{\text {weak }}$ data analysis, specifically the analysis of the contribution from the aluminum target walls, which provides $30 \%$ of the measured signal. This thesis also focuses on the subpercent precision determination of the electron beam polarization, reporting the first sub-percent Møller polarimeter results from Jefferson Laboratory Hall C.

This chapter focuses on the theoretical background of the Standard Model, emphasizing the electroweak sector. Chapters 2 and 3 focus on the experimental technique of parity-violating electron scattering, and introduce the $Q_{\text {weak }}$ experiment. Chapter 4 reports on the first sub-percent precision results from the Hall C Møller polarimeter, while Chapters 5 and 6 detail the analysis of the background from the $Q_{\text {weak }}$ target's aluminum walls. 


\subsection{Introduction to the Standard Model}

The Standard Model of Particle Physics (SM) is a unique, self-consistent, re-normalizable, gauge theory. Of the four known fundamental forces (strong nuclear force, weak nuclear force, electromagnetism, and gravity), it describes three; only gravity is absent. Mathematically, it combines the strong gauge theory of color, $S U_{c}(3)$, with the minimal unified $S U_{L}(2) \times U_{Y}(1)$ gauge group proposed by Glashow [3], Weinberg [4], and Salam [5] in the 1960's.

The Standard Model has powerful predictive ability. Over the past several decades a vigorous experimental program has repeatedly upheld its theoretical foundations. Relatively recent findings, such as neutrino mass mixing, have required modifications to the originally proposed Standard Model, but the underlying fundamental structure remains on solid footing.

Despite these successes, the Standard Model is incomplete. Gravity is conspicuously absent, and no candidates exist to explain dark matter or dark energy. It does not contain nearly enough $C P$ violation to explain the stark contrast between the matter and anti-matter density in the universe, and it provides no insight to the large discrepancy between the interaction strengths of the various forces (known as the hierarchy problem).

In the Standard Model, fundamental particles are classified into two types: bosons (integer spin) obey Bose-Einstein statistics, and fermions (half-integer spin) obey FermiDirac statistics. Bosons are the force carriers, or interaction mediators, between particles, and come directly from the gauge group generators. Photons $(\gamma$ 's) are the mediators of the familiar electromagnetic force, while $W^{+}, W^{-}$, and $Z^{0}$ bosons mediate the charged and neutral weak interactions, respectively. Unlike the photon, the other three mediators $\left(W^{+/-}\right.$and $\left.Z^{0}\right)$ are all massive $(\sim 90 \mathrm{MeV})$. This results in the weak force having a limited range. Finally, one fundamental scalar particle exists (spin-0): the renowned, recently discovered Higgs boson [6]. The Higgs sector is responsible for the fermionic masses, and is the subject of Section 1.3.

Electrons and quarks are familiar fundamental fermions, while protons and neutrons are composite fermions. Fermion interactions are described by the transformation properties of the underlying gauge group. These transformation properties are relatively complicated, with three generations, or copies, of particles. Each generation couples identically to the spin-one mediators. The first generation is composed of up $(u)$ and down $(d)$ type quarks, the electron, and the electron-neutrino. 
Figure 1.1 summarizes the Standard Model particle content. Three generations of fermions are positioned at left, with the force mediators listed on the right. The top two fermion rows are quarks, while the bottom two are leptons. The fermions in each row all share fundamental quantum numbers (spin, charge, and baryon or lepton number), but differ in mass. Each lepton generation is composed of a particle pair: a charged lepton $(e, \mu$, and $\tau)$ and a partner neutrino.

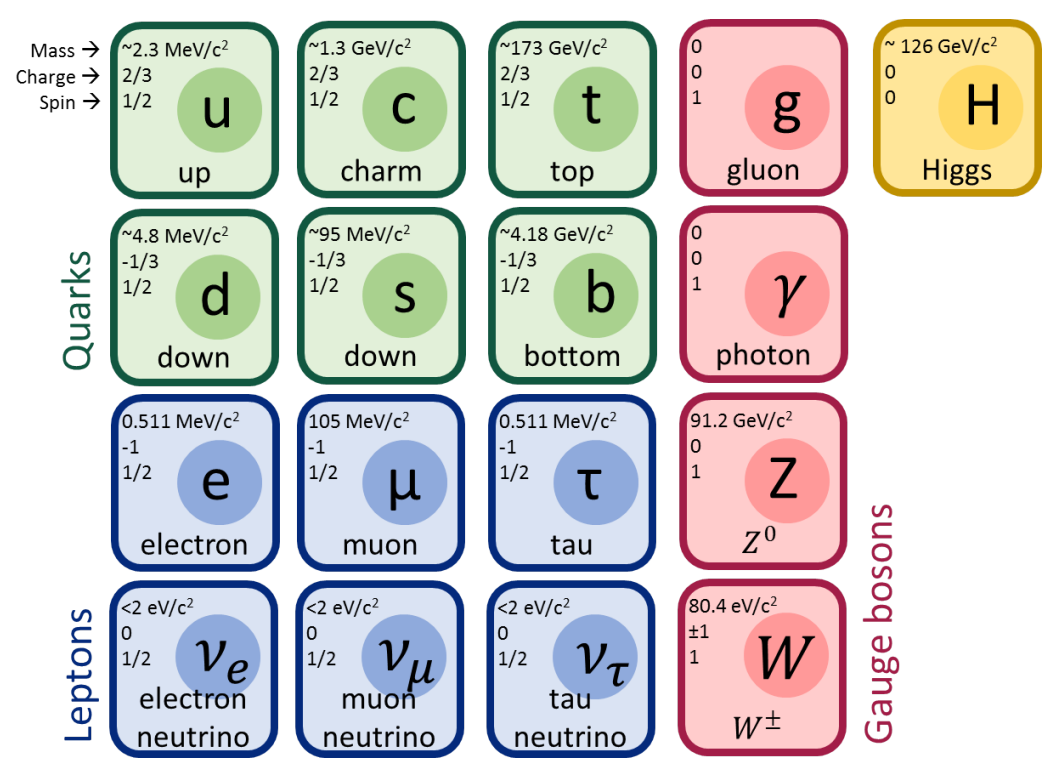

Figure 1.1: Particle content of the Standard Model, with each fundamental particle mass, charge, and spin. Quarks (green) and leptons (blue) are fermions, while the force mediators (red) and the scaler Higgs (yellow) are bosons.

Only quarks and gluons carry color charge and participate in strong interactions, such as binding quarks together. Strongly interacting phenomena display some interesting properties, notably confinement. Whereas all other known interactions decrease in strength with distance, the strong force actually increases. This leads to quarks being tightly bound in color-singlet states, and are therefore not directly observed isolated in nature. Quarks can bind into groups of two (mesons) or three (baryons). ${ }^{1}$ Protons and neutrons are both examples of baryons, with the proton composed of two up quarks and a down quark $(p=u u d)$, while the neutron consists of two down quarks and an up quark $(n=u d d)$. Quarks also participate in the electromagnetic and weak interactions.

Leptons only interact weakly and electromagnetically. One important feature of the Standard Model is the unification of electromagnetic and weak interactions. This is described in detail in Section 1.3, and is one of the most rigorously tested pieces

\footnotetext{
${ }^{1} \mathrm{~A}$ colorless five-quark combination (the pentaquark) is allowed in the Standard Model, and has a capricious history; several reported discoveries were later retracted. However, the Large Hadron Collider at CERN recently claimed discovery of two channels with a significance of over $9 \sigma[7]$.
} 
of the theory. It also provided a rich theoretical framework for a rigorous series of experimental tests throughout the last generation, including parity-violating electron scattering, which is a main focus of this thesis.

\section{$1.2 \quad$ Symmetries}

Symmetries are an important feature of the Standard Model, providing deep insight into fundamental structure. In discussing the Standard Model we are interested in dynamical symmetries, beyond the simple static symmetries often discussed (e.g., symmetries in equations of motion versus symmetries in crystal lattices). Noether's theorem shows a profound relationship between these symmetries and conservation laws; namely that for each global, dynamical symmetry there is an associated conserved quantity [8]. For example, systems which remain invariant after Poincaré transformations (linear and rotational transformations) conserve linear and angular momentum. Equations symmetric in time conserve energy.

Moreover, the symmetries in the Standard Model are internal symmetries, which leave the Lagrangian invariant. Consider the Lagrangian for a spin $1 / 2$ particle of mass $m$,

$$
\mathcal{L}=\bar{\psi}\left(i \gamma^{\mu} \partial_{\mu}-m\right) \psi
$$

where $\psi$ is the particle wave function, $\mu$ is a Lorentz index $\left(\mu=(t,-\bar{x})\right.$, and $\gamma^{\mu}$ are the Dirac gamma matrices. This Lagrangian is invariant under a global phase shift, $\alpha$, as:

$$
\psi \rightarrow e^{-i q \alpha} \psi
$$

That is, the equations of motion (the Dirac equation) for this particle remain unchanged. This phase invariance implies conservation of charge (in this case, $q$ ).

In general, symmetries that act on spacetime coordinates and fields cannot leave the Lagrangian invariant, as the Lagrangian is not constant in spacetime coordinates [9]. Therefore, imposing a local spacetime dependent gauge, such as

$$
\psi \rightarrow e^{-i q \alpha(x)} \psi,
$$

manifestly destroys the invariance because the gauge field derivatives do not vanish:

$$
\partial_{\mu} \psi=e^{-i q \alpha(x)}\left[\partial_{\mu}-i q\left(\partial_{\mu} \alpha(x)\right)\right] \psi
$$

It is possible to impose local gauge invariance by defining a gauge-covariant derivative, $\mathcal{D}_{\mu}$, in terms of an associated gauge field, $A_{\mu}$, as

$$
\partial_{\mu} \rightarrow \mathcal{D}_{\mu} \equiv \partial_{\mu}-i q A_{\mu}
$$


The gauge field, $A_{\mu}$, transforms under the same local spacetime-dependent gauge transformation, such that the unwanted term in equation (1.4) vanishes:

$$
A_{\mu} \rightarrow A_{\mu}+\frac{1}{q} \partial_{\mu} \alpha(x)
$$

Clearly, imposing a global invariance to hold locally is a strong condition. Physically, the introduction of gauge fields injects an additional particle. In this case, forcing a global phase invariance to hold locally reproduces electrodynamics with the (massless) photon as the gauge field.

This concept, forcing a global symmetry to hold locally, underpins a large part of the base Standard Model. However, it fails to explicitly produce the mass terms needed in a full, functional electroweak theory. These in turn, are introduced via the Higgs mechanism in Section 1.3.

\subsubsection{Discrete symmetries}

There are two main classes of symmetries, continuous and discrete. Parity is an example of a discrete symmetry, and is the inversion of all spatial coordinates (a mirror reflection). Given a vector state, $|V\rangle$,

$$
|V\rangle=\left(\begin{array}{l}
x \\
y \\
z
\end{array}\right)
$$

the parity operator inverts its components:

$$
\mathcal{P}|V\rangle=\left(\begin{array}{c}
-x \\
-y \\
-z
\end{array}\right)
$$

Vectors are good parity eigenstates with eigenvalue -1 .

When looking in a mirror, one's right and left hands are interchanged. This concept is extended to particles by defining them a particular handedness. For a massive particle in the relativistic limit, its helicity, $h$, is defined as the product of a particle's spin vector, $\mathbf{s}$, with momentum direction, $\hat{\mathbf{p}}$. If they are aligned the particle has positive helicity, while if anti-aligned it has negative helicity:

$$
h \equiv \mathbf{s} \cdot \hat{\mathbf{p}}= \begin{cases}>0 & \text { positive helicity } \\ <0 & \text { negative helicity }\end{cases}
$$

Helicity is a good quantum eigenstate, with eigenvalues of $\pm^{1} / 2$. However, helicity is not Lorentz invariant for massive particles, as boosting to different reference frames may switch the eigenvalue. The handedness of massless particles, called chirality, is a Lorentz 
invariant, and therefore an intrinsic property of the particle. For massive particles in the ultrarelativistic limit, helicity approaches chirality. This distinction becomes important in subsequent discussions, when parts of Standard Model only engage with left-handed states.

Until 1956, it was widely assumed that parity was a good symmetry and held in all interactions; that is, the mirror image of all physical processes were also physically possible. Vast evidence existed that both strong and electromagnetic interactions respected parity, but a review by T.S. Lee and C.N. Yang found no known direct experimental confirmation of parity conservation in weak decays [10]. They proposed a number of experimental tests, convincing their colleague C.S. Wu to perform her now famous experiment [11]. In the experiment, she observed the beta-decay of ${ }^{60} \mathrm{Co}$, in the reaction ${ }^{60} \mathrm{Co} \rightarrow{ }^{60} \mathrm{Ni}+e+\bar{\nu}_{e}$. In a technically challenging experiment, she aligned the nuclear spins, and measured the direction of the emitted electrons. The electron direction was predominately in the opposite direction of nuclear spin, a stark violation of parity because in the mirror world, the nuclear handedness flips, but the direction of the emitted electron does not.

Since this experiment, parity violation has essentially become the signature of the weak force. This occurs because the weak interactions mix parity eigenstates. Scaler, pseudoscalar, vector, and axial-vector interactions are all good eigenstates of parity separately, but not necessarily when they mix. For example, given good vector and axial-vector eigenstates, $|V\rangle$ and $|A V\rangle$, the parity-eigenvalue will be -1 and +1 , respectively:

$$
\begin{gathered}
\mathcal{P}|V\rangle=-|V\rangle \\
\mathcal{P}|A V\rangle=+|A V\rangle .
\end{gathered}
$$

Mixed states, such as $(|V\rangle+|A V\rangle)$, are not eigenstates of good parity,

$$
\begin{aligned}
\mathcal{P}(|V\rangle+|A V\rangle) & =-|V\rangle+|A V\rangle \\
& \neq \pm(|V\rangle+|A V\rangle) .
\end{aligned}
$$

As becomes clear in Section 1.3, the weak interaction vertex is governed by a $V-A$ structure that violates parity. In the underlying gauge theory, this occurs by separating left-handed fermions into a doublet and right-handed fermions into a singlet state, which are described in more detail in Section 1.3.1. This parity-violating vertex is the focus of the rich parity program at Jefferson Lab and, specifically of the $Q_{\text {weak }}$ experiment, which this thesis focuses on. 


\subsection{Electroweak unification}

The $S U_{L}(2) \times U_{Y}(1)$ structure of the electroweak Standard Model was developed in the 1960s and 1970s by Glashow, Weinberg, and Salam, and quickly put on firm experimental footing. It unified both the electromagnetic and weak interactions into one theoretical framework. Interactions come from rotations of the gauge group generators, while the Higgs mechanism provides a mass generating mechanism for the three massive gauge bosons $[12,13,14]$. It also allows for massive fermions if one assumes a simple Yukawa coupling, all while leaving the Standard Model renormalizable.

This section begins by introducing some characteristics of the $S U_{L}(2)$ and $U_{Y}(1)$ gauge fields, then continues with an overview of the Higgs mechanism (spontaneous symmetry breaking coupled with the enforcing of local gauge invariance) in Section 1.3.2. This entire section assumes some familiarity with field theory and basic group structure. I will primarily follow the development of $[9,15]$, however the interested reader is directed to the literature for further introduction $[16,17]$ or discussion $[18,19]$.

\subsubsection{Electroweak gauge structure}

Naively one might expect the electromagnetic and weak interactions to be completely separate, assuming $S U_{L}(2) \equiv S U_{W}(2)$ and $U_{Y}(1) \equiv U_{E M}(1)$. However, doing so would produce four massless gauge bosons, each related to the gauge group generators. Experiments long ago excluded such a possibility. One possible solution is introducing the gauge boson mass terms explicitly by hand. Although there is no a priori reason to exclude this option, it produces a non-renormalizable result, effectively destroying all predictive power [15]. Instead, it is possible to generate an alternate theory which mixes $S U_{L}(2) \times U_{Y}(1)$ and through spontaneous symmetry breaking results in the familiar $U_{E M}(1)$ symmetry.

Although the means for a specific proof have not been introduced, we can anticipate the form of the $S U_{L}(2)$ and $U_{Y}(1)$ currents from their gauge structure. Recall the electromagnetic current, $J_{\mu}^{e m}$, from QED. It is given in terms of its coupling, $e$, and contracts with its gauge field, $A_{\mu}$, and interacts with spin- $1 / 2$ particles, $\psi$, as

$$
J_{\mu}^{e m} A^{\mu}=\left(\bar{\psi} \gamma_{\mu} Q \psi\right) A^{\mu}
$$

The generator of the $U_{E M}(1)$ gauge group, $Q$, is the charge.

The $U_{Y}(1)$ gauge group is fundamentally similar to the electromagnetic result. It introduces a new current, $J_{\mu}^{Y}$, and gauge field, $B^{\mu}$. Its generator is hypercharge, $Y$, 
and coupling is $g_{1}$. These are related through

$$
-i \frac{g_{1}}{2} J_{\mu}^{Y} B^{\mu}=-i g_{1} \bar{\psi} \gamma_{\mu} \frac{Y}{2} \psi B^{\mu} .
$$

The factor of $1 / 2$ is conventional.

The expression is fundamentally similar for the larger $S U_{L}(2)$ gauge group. The $S U_{L}(2)$ group has an isotriplet of weak currents $J_{\mu}^{a}(a=1,2,3)$ coupled to three gauge fields $W_{\mu}^{a}$ through its gauge group generators $\tau^{a}$, which are the familiar Pauli matrices. Its coupling is given as $g_{2}$ :

$$
-i g_{2} J^{\mu, a} W_{\mu}^{a}=-i g_{2} \bar{\chi}_{L} \gamma_{\mu} \tau^{a} W^{\mu, a} \chi_{L}
$$

The $\chi_{L}$ are left-handed fermion weak isospin doublets mentioned at the end of Section 1.2.1. The subscript L reminds us that they only interact with left-handed particles. The right-handed fermions are weak isosinglets and are decoupled from this sector. For example, the electron and its associated neutrino combine into a left-handed isodoublet, but only the right-handed electron appears in the isosinglet:

$$
\chi_{L}=\left(\begin{array}{c}
\nu_{e} \\
e^{-}
\end{array}\right), \text {and } \psi_{R}=e_{R}^{-} .
$$

Since the right- and left-handed particles interact differently in the theory, it's often helpful to define the chiral projection operators, $P_{R}$ and $P_{L}$.

$$
P_{L}=\frac{1}{2}\left(1-\gamma_{5}\right), \quad \text { and } \quad P_{R}=\frac{1}{2}\left(1+\gamma_{5}\right) .
$$

These also select only the right- and left-hand components of a state, respectively. For example, a left-handed fermion is written

$$
\psi_{L} \equiv \frac{\left(1-\gamma^{5}\right)}{2} \psi
$$

Using these chiral projection operators, the vertex structure of weak interactions (vector minus axial-vector, or $V-A$ ) is manifest.

The gauge group generators, and thus the charges, are all linked by the Gell-MannNishijima relation:

$$
Q=T^{3}+\frac{Y}{2},
$$

where $T^{3}$ is the third-component of weak isospin. Likewise, the electroweak currents are related by

$$
J_{\mu}^{e m}=J_{\mu}^{3}+\frac{1}{2} J_{\mu}^{Y} .
$$

Through this relation it's clear that the electromagnetic sector is completely contained within the larger, mixed group structure. It contains both right- and left- handed 
components (remember, $J_{\mu}^{3}$ does not contain both). Thus the charged weak interactions appear to only interact with left-handed fermions, while the neutral interactions interact with both species.

\subsubsection{Spontaneous symmetry breaking and the Higgs mechanism}

As suggested in Section 1.2, enforcing a global symmetry to hold locally produces a massless gauge field. Although not explicitly shown here, this is a general result. Consequently, imposing local symmetries on the $S U_{L}(2) \times U_{Y}(1)$ group will produce four massless gauge fields (a $W_{\mu}^{a}$ triplet and a $B_{\mu}$ singlet), not the experimentally verified massive $W^{+}, W^{-}$, or $Z^{0}$. The Higgs mechanism combines imposing local gauge invariance with spontaneous breaking of the potential's symmetry; when these combine the resulting Lagrangian trades the massless field for a massive one.

The pertinent part of the electroweak Lagrangian is the kinetic term, $\mathcal{L}_{k i n}$, where the Higgs couples to the $S U_{L}(2)$ and $U_{Y}(1)$ fields. The scalar Higgs field, $\phi$, has the usual kinetic and potential terms:

$$
\mathcal{L}_{\text {kin }}=\left(\partial_{\mu} \phi\right)^{\dagger}\left(\partial^{\mu} \phi\right)-V\left(\phi^{\dagger} \phi\right)-\mathcal{L}_{h f}
$$

$\mathcal{L}_{h f}$ are the fermion-Higgs Yukawa type couplings. These Yukawa couplings are shown later to produce fermion masses.

First, we impose local gauge invariance by defining the gauge covariant derivative, $D_{\mu}$, as

$$
D_{\mu} \phi=\partial_{\mu} \phi-\frac{i g_{2}}{2} W_{\mu}^{a} \tau^{a} \phi-\frac{i g_{1}}{2} Y B_{\mu} \phi .
$$

Now we can show a spontaneous broken symmetry produces the requisite mass terms. We start by finding a global minimum and then breaking the symmetry. The Higgs potential is given in terms of two scalars, $\mu$ and $\lambda$,

$$
V\left(\phi^{\dagger} \phi\right)=\mu^{2} \phi^{\dagger} \phi+\lambda\left(\phi^{\dagger} \phi\right)^{2}
$$

This potential is sometimes called the Mexican hat potential. To keep the Lagrangian invariant, the Higgs fields must belong to $S U_{L}(2) \times U_{Y}(1)$ multiplets with weak hypercharge $Y=1$. A convenient choice is a weak isospin doublet made of complex scalar fields:

$$
\boldsymbol{\Phi}=\left(\begin{array}{c}
\phi^{+} \\
\phi^{0}
\end{array}\right) \text { with } \begin{aligned}
& \phi^{+}=\left(\phi_{1}+i \phi_{2}\right) / \sqrt{2} \\
& \phi^{0}=\left(\phi_{3}+i \phi_{4}\right) / \sqrt{2}
\end{aligned}
$$

This potential is minimized when $\mu^{2}<0$;

$$
\langle\boldsymbol{\Phi}\rangle^{2}=\frac{v}{\sqrt{2}} \equiv \sqrt{\frac{\mu^{2}}{2 \lambda}} .
$$


The vacuum expectation value, $v$ is fixed by the Fermi constant, and is $\sim 246 \mathrm{GeV}[20]$.

To break the symmetry, we must now choose an explicit gauge condition for the field $\boldsymbol{\Phi}$. Choosing the vacuum expectation value breaks the $S U_{L}(2) \times U_{Y}(1)$ symmetry, ultimately leaving the $U_{E M}(1)$ intact:

$$
\boldsymbol{\Phi}=\frac{1}{\sqrt{2}}\left(\begin{array}{l}
0 \\
v
\end{array}\right)
$$

Now we can re-write the Lagrangian in (1.18) using (1.23). The first term (originally $\left.\left(\partial_{\mu}\right)^{\dagger}(\partial)^{\mu}\right)$, now becomes

$$
\begin{aligned}
\left(D_{\mu} \phi\right)^{\dagger}\left(D^{\mu} \phi\right) & =-\frac{g_{2}^{2} v^{2}}{8}\left|W_{\mu}^{1}-i W_{\mu}^{2}\right|^{2}-\frac{v^{2}}{8}\left(-g_{2} W_{\mu}^{3}+g_{1} B_{\mu}\right) \\
& =-\frac{g_{2}^{2} v^{2}}{4} W_{\mu}^{+} W^{-\mu}-\frac{v^{2}}{8}\left(g_{1}^{2}+g_{2}^{2}\right) Z_{\mu} Z^{\mu}+0 A_{\mu} A^{\mu}
\end{aligned}
$$

where the following convenient definitions have been used

$$
W_{\mu}^{ \pm}=\frac{1}{\sqrt{2}}\left(W_{\mu}^{1} \mp i W_{\mu}^{2}\right), \quad Z_{\mu}=\frac{-g_{1} W_{\mu}^{3}+g_{2} B_{\mu}}{\sqrt{g_{1}^{2}+g_{2}^{2}}}, \quad A_{\mu}=\frac{g_{1} W_{\mu}^{3}+g_{2} B_{\mu}}{\sqrt{g_{1}^{2}+g_{2}^{2}}} .
$$

The $W_{\mu}^{ \pm}$correspond to the charged-current interactions, while $Z_{\mu}$ and $A_{\mu}$ correspond to the neutral weak and electromagnetic interactions, respectively. Masses of the physical gauge bosons are immediately accessible as the coefficients of terms quadratic in the fields:

$$
m_{W^{ \pm}}=m_{W^{1,2}}=\frac{g_{2} v}{2}, \quad m_{Z}=\sqrt{\frac{\left(g_{1}^{2}+g_{2}^{2}\right) v^{2}}{4}}, \quad m_{\gamma}=0 .
$$

The predicted masses of the charged and neutral-weak boson show fine agreement with experimentally measured values of $m_{W} \sim 80.4 \mathrm{GeV} / c^{2}$ and $m_{Z} \sim 91.2 \mathrm{GeV} / c^{2}[20]$.

In particular, note the photon and neutral-weak $Z^{0}$ are orthogonal mixtures of the $W^{3}$ and $B$ gauge fields. To simplify the notation and make the relationship manifest, we define the weak mixing angle

$$
\tan \left(\theta_{W}\right)=\frac{g_{2}}{g_{1}} \quad \text { or } \quad \sin ^{2}\left(\theta_{W}\right)=\frac{g_{2}^{2}}{g_{1}^{2}+g_{2}^{2}} .
$$

The weak mixing angle, or Weinberg angle, is an important part of the Standard Model, and characterizes the amount of electroweak mixing in the theory. Precise, low energy determinations of $\sin ^{2} \theta_{W}$ are a fundamental probe of the Standard Model, including $Q_{\text {weak }}$.

Ignoring couplings, the physical gauge boson states can be concisely related through a mixing matrix as

$$
\left(\begin{array}{c}
A_{\mu} \\
Z_{\mu}
\end{array}\right)=\left(\begin{array}{cc}
\sin \theta_{W} & \cos \theta_{W} \\
\cos \theta_{W} & -\sin \theta_{W}
\end{array}\right)\left(\begin{array}{c}
W_{\mu}^{3} \\
B_{\mu}
\end{array}\right) .
$$


Mass terms for the fermions also generate from the Higgs sector. If we add a perturbation around the vacuum expectation value,

$$
\boldsymbol{\Phi} \rightarrow\left(\begin{array}{c}
0 \\
v+H
\end{array}\right)
$$

the above formalism remains intact, but now one component of the fermion $S U_{L}(2)$ doublets has a Higgs coupling. Fermion mass terms are proportional to this coupling. The neutrinos, lacking a right handed singlet state, remain massless under this approach. The addition of neutrino masses to the Standard Model is beyond the scope of this document.

Although an ingenious technique, the Higgs sector actually parameterizes most of our ignorance of the electroweak Standard Model. The Standard Model has at minimum 19 free parameters; the Higgs sector introduces 15 of them (nine fermion masses, the Higgs mass, the Higgs self-coupling, and four angles parameterizing quark-flavor mixing) [9]. This is one reason the Higgs sector is an active sector for new physics searches.

\subsubsection{Neutral current interactions}

The simplest neutral-current interactions include exchange of either a massless photon, $\gamma$, or a massive $Z^{0}$ boson. The photon current was given in equation (1.10). The neutral- $Z^{0}$ exchange current can be essentially given in terms of the fundamental weak and hypercharge currents in proportion to the mixing from equation (1.28):

$$
J_{\mu}^{Z}=W_{\mu}^{3} \cos \theta_{W}-J_{\mu}^{Y} \sin \theta_{W}
$$

Solving equation (1.16) for the $Y$, and substituting equations (1.11), (1.12), and (1.14) into (1.30) yields

$$
J_{\mu}^{Z}=\frac{e}{2 \sin \theta_{W} \cos \theta_{W}}\left[\bar{\psi}_{L}\left(2 T^{3}-2 Q \sin ^{2} \theta_{W}\right) \gamma_{\mu} \psi_{L}+\left(-2 Q \sin ^{2} \theta_{W}\right) \bar{\psi}_{R} \gamma_{\mu} \psi_{R}\right] .
$$

The coefficient, $e / 2 \sin \theta_{W} \cos \theta_{W}$ is the intrinsic $Z^{0}$ coupling strength, $g_{Z}$. To simplify the notation, we define the weak axial and vector charges for a fermion, $f$, as

$$
\begin{aligned}
& g_{V}^{f}=2 T_{f}^{3}-4 Q_{f} \sin ^{2} \theta_{W} \\
& g_{A}^{f}=2 T_{f}^{3} .
\end{aligned}
$$

Now the $Z^{0}$ current is

$$
J_{\mu}^{Z}=g_{Z} \bar{\psi} \gamma_{\mu}\left(g_{V}^{f}-g_{A}^{f} \gamma^{5}\right) \psi
$$


Using this formalism the theory's $V-A$ (vector minus axial-vector) structure is manifest. They are written for a general fermion, $f$, of given charge $Q^{f}$ and weak isospin $J_{\mu}^{3}$. Table 1.1 summarizes these results for the various fundamental fermions. From these results, the vertex factor and propagator of the $Z^{0}$ exchange can also be written.

\begin{tabular}{|l|c|c|c|r|}
\hline Fermion type & $T^{3}$ & $\mathrm{Q}$ & $g_{V}$ & $g_{A}$ \\
\hline$\nu_{e}, \nu_{\mu}, \nu_{\tau}$ & $+\frac{1}{2}$ & 0 & 1 & 1 \\
\hline$e^{-}, \mu^{-}, \tau^{-}$ & $-\frac{1}{2}$ & -1 & $-1+4 \sin ^{2} \theta_{W}$ & -1 \\
\hline$u, c, t$ & $+\frac{1}{2}$ & $+\frac{2}{3}$ & $1-\frac{8}{3} \sin ^{2} \theta_{W}$ & 1 \\
\hline$d, s, b$ & $-\frac{1}{2}$ & $-\frac{1}{3}$ & $-1+\frac{4}{3} \sin ^{2} \theta_{W}$ & -1 \\
\hline
\end{tabular}

Table 1.1: Neutral vector and axial-vector couplings in the Standard Model.

\subsubsection{Nucleon weak charges}

With the formalism now developed, it's possible to derive simple theoretical expressions for the lowest-order nucleon charges. The full experimental description, which includes nucleon form factors, is delayed until Chapter 2.

The electromagnetic and neutral-weak charges of composite structures can be determined from equations (1.33) via the coherent sum of the vector currents listed in Table 1.1. For example, a proton has quark content: uud. The familiar proton's electromagnetic charge is given by

$$
\begin{aligned}
Q_{p}^{E M}=\sum Q_{i} & =Q_{u}+Q_{u}+Q_{d} \\
& =2 / 3+2 / 3-1 / 3 \\
& =1 .
\end{aligned}
$$

Likewise, the proton's neutral-weak charge (to first order) is

$$
\begin{aligned}
Q_{p}^{Z}=Q_{W}^{p} & =\sum Q_{i}^{Z}=Q_{u}^{Z}+Q_{u}^{Z}+Q_{d}^{Z} \\
& =\left(1-\frac{8}{3} \sin ^{2} \theta_{W}\right)+\left(1-\frac{8}{3} \sin ^{2} \theta_{W}\right)+\left(-1-\frac{4}{3} \sin ^{2} \theta_{W}\right) \\
& =1-4 \sin ^{2} \theta_{W} .
\end{aligned}
$$

The current experimentally determined value for $\sin ^{2} \theta_{W} \approx 0.23$ [20], suggesting the proton's weak charge is $\approx 0.07$. Curiously, the neutron's electroweak charges sharply contrast with the protons: the neutron electric charge is of course 0 , but its predicted weak charge is identically -1 . This has serious implications for electron scattering experiments with proton or helium targets, as neutral-weak exchange greatly favors neutrons. This is discussed more in Chapter 2, and the experimental challenges from this fact are the subjects of Chapters 5 and 6 . 
Likewise, the weak charges of larger nuclei can similarly be determined. For electron scattering experiments, the general neutral-current Lagrangian, $\mathcal{L}_{n c}$, can be written as

$$
\mathcal{L}_{n c}=\frac{G_{F}}{\sqrt{2}} \sum_{q}\left(C_{1 q} \bar{e} \gamma_{\mu} \gamma_{5} e \bar{q} \gamma^{\mu} q+C_{2 q} \bar{e} \gamma_{\mu} e \bar{q} \gamma^{\mu} \gamma_{5} q\right)
$$

Here the $C_{1 q}$ and $C_{2 q}$ the quark vector and axial-vector couplings for quark flavor $q$ (these differ from $g_{V}$ only by a normalization factor). Note that in the $C_{1}$ term the helicity dependence is in the electron vertex, and also that the $C_{2}$ 's are not conserved. The non-conservation of axial currents has implications when parameterizing form factors.

For a proton, the weak charge can also be written as a coherent sum of the vector quark couplings, $C_{1 u}$ and $C_{1 d}$ :

$$
Q_{W}^{p}=-2\left(2 C_{1 u}+C_{1 d}\right)
$$

where the initial factor of 2 is convention. A nucleus with $Z$ protons and $N$ neutrons would have a weak charge of

$$
Q_{W}=-2\left[C_{1 u}(2 Z+N)+C_{1 d}(Z+2 N)\right]
$$

\subsection{Electroweak radiative corrections}

The results derived thus far have only been for leading order processes (tree level); higher-order effects have been ignored. This section presents the basics of these higher order corrections, in particular the pieces relevant to the low-energy parity-violating program at JLab.

One important Standard Model prediction is that interaction couplings actually vary with the probe's momentum transfer, $Q^{2}$. This is a well-known phenomenon in QED, where higher order terms noticeably contribute to scattering processes. Perhaps the most striking example is the vacuum polarization contribution (Figure 1.2, right). The internal photon loop momentum is not constrained via conservation of energy or momentum, and thus an ultraviolent divergence occurs. To constrain the theory, a cutoff energy is specified, and the divergent piece is absorbed by the coupling constant. For electromagnetic interactions the coupling is the usual $\alpha \sim 1 / 137$. However, after accounting for this divergence, $\alpha$ becomes a function of $Q^{2}$ (that is, $\alpha$ is renormalized to $\alpha\left(Q^{2}\right)$, meaning the strength of charged interactions are now $Q^{2}$-dependent). During low-energy scatters the bare charge in the Lagrangian is screened by this effect. We say the coupling runs with $Q^{2}$. This is experimentally seen in the hyperfine structure of the hydrogen spectrum (the Lamb shift). 


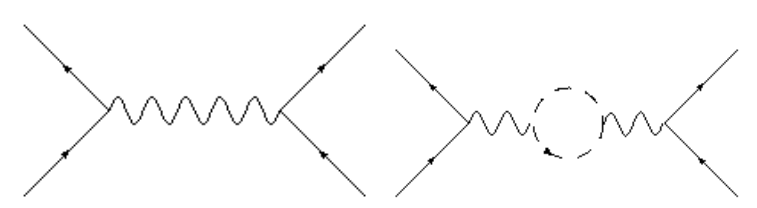

Figure 1.2: Two particles interact through exchange of a photon. (Left) Tree level process. (Right) Higher order effect with a fermion loop in the photon propagator.

Two categories of electroweak radiative corrections exist. They may be either pure QED or electroweak. QED corrections include corrections to the incoming and outgoing electron vertices, including bremsstrahlung. These shift the effective interaction $Q^{2}$, and therefore the scattering process kinematics. QED radiative effects are well understood (e.g., see [21]), and are outside the scope of this document.

Similar to the vacuum polarization in QED, electroweak radiative corrections cause an evolution of $\sin ^{2} \theta_{W}$ with $Q^{2}$ [22]. Figure 1.3 shows this running in the $\overline{\mathrm{MS}}$ renormalization scheme [22]. These radiative corrections are well understood; the theoretical uncertainty is the line thickness. The electroweak vacuum polarizations include internal loops from the individual fermions. As the mass threshold to produce them is crossed, the line kinks ${ }^{2}$. This explains the flat behavior at low- $Q$, which progressively bends with the addition of each quark-type, until the final spike at the $W$-pole. ${ }^{3}$ The resultant shift in $\sin ^{2} \theta_{W}$ from the $Z^{0}$-pole to low- $Q$ is approximately $\sim 0.007$. The complete calculation details were recently made available in [24].

The weak charge of the proton, $Q_{W}^{p}$, is directly related to the weak mixing angle at tree level, as seen in equation (1.36). Including radiative corrections, the proton's weak charge is related to the weak mixing angle by [25]

$$
Q_{W}^{p}=\left[\rho_{N C}+\Delta_{e}\right]\left[1-4 \sin ^{2} \hat{\theta}_{W}(0)+\Delta_{e}^{\prime}\right]+\square_{W W}+\square_{Z Z}+\square_{\gamma Z}
$$

The radiative corrections can be separated into three main categories, shown in Figure 1.4. The first correction, $\rho_{N C}$ is a correction to the ratio of the neutral-tocharged current interaction strength,

$$
\rho_{N C} \equiv \frac{g_{W}^{2}}{g_{Z}^{2}}
$$

In the literature, $\rho_{N C}$ is often written in terms of the Fermi coupling constant, or the ratio of $W$ to $Z^{0}$ mass. Regardless, to first order $\rho_{N C} \sim 1.00$.

\footnotetext{
${ }^{2}$ This characteristic feature is an artifact from using hard mass cutoffs in the calculation. Other alternate calculations avoid this approximation, leading to a visually modified, smooth curve. See $[23,24]$, for example.

${ }^{3}$ The final spike occurs at the $W$-pole from an interaction where $Z^{0}$ goes to a $W W$ loop, which annihilate to $\gamma$. The pure $Z^{0}$ equivalent, $Z^{0}$ goes to $Z^{0} Z^{0}$ requires a triple $Z^{0}$ vertex which is forbidden.
} 


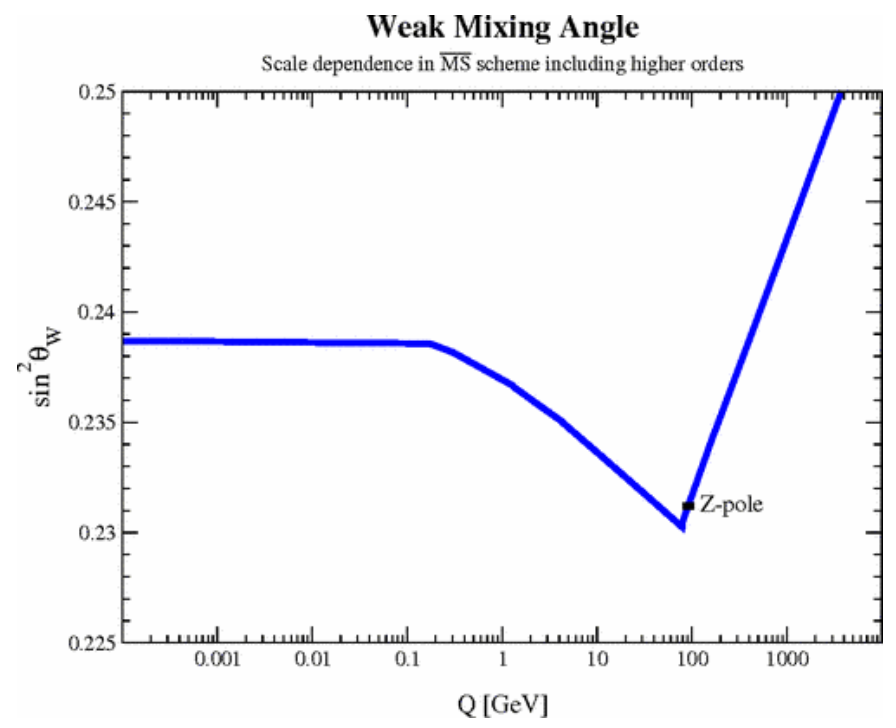

Figure 1.3: Theoretical evolution of the weak mixing angle vs. $Q\left(\sqrt{Q^{2}}\right)$ in the $\overline{\mathrm{MS}}$ renormalization scheme [22].

The second group are parity-violating modifications to the external electron legs, as seen in Figure 1.4 center. $\Delta_{e}$ is a photon loop correction to the external legs of $Z^{0}$ exchange, while $\Delta_{e}^{\prime}$ is the reverse: a $Z^{0}$-boson loop correction to the external legs of photon exchange. Both of these corrections to $\sin ^{2} \theta_{W}$ at $Q^{2}=0$ are rather small, $<1 \%$.
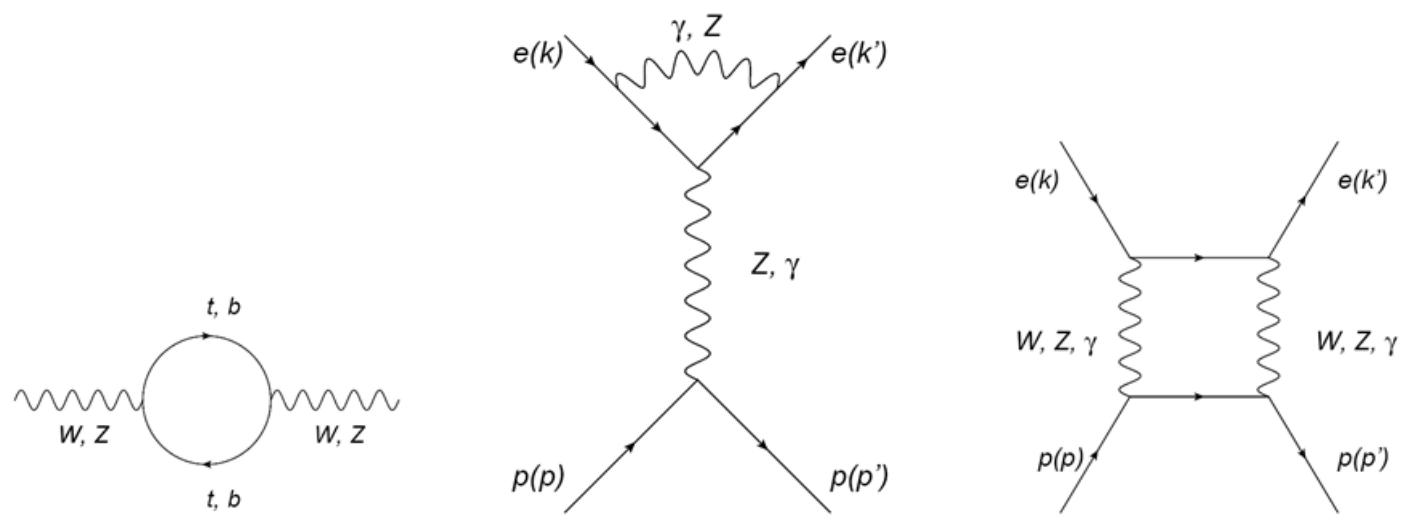

Figure 1.4: Three primary categories of electroweak radiation corrections for the proton's weak charge. (Left) Ratio of neutral/charged weak exchange, $\rho_{N C}$. (Center) External leg corrections. (Right) Box diagrams.

The final set of corrections are the box diagrams, of which there are three (Figure 1.4, right). Although large, the $\square_{W W}$ and $\square_{Z Z}$ box are both directly calculable to high precision through perturbation theory due to the large mass of the $W^{ \pm}$and $Z^{0}$ bosons. The $\square_{W W}$ correction is approximately $12 \%$. However, perturbation theory fails for the $\gamma-Z^{0}$ interference box, due to the massless photon leg. 
Similar to the neutral current Lagrangian, the $\square_{\gamma Z}$ correction can be split into two components depending on the couplings: vector-electron $\times$ axial-proton, or axialelectron $\times$ vector-proton. Recent calculations of the vector-electron contribution show a slight energy dependence, shown in Figure 1.5 (red curve).

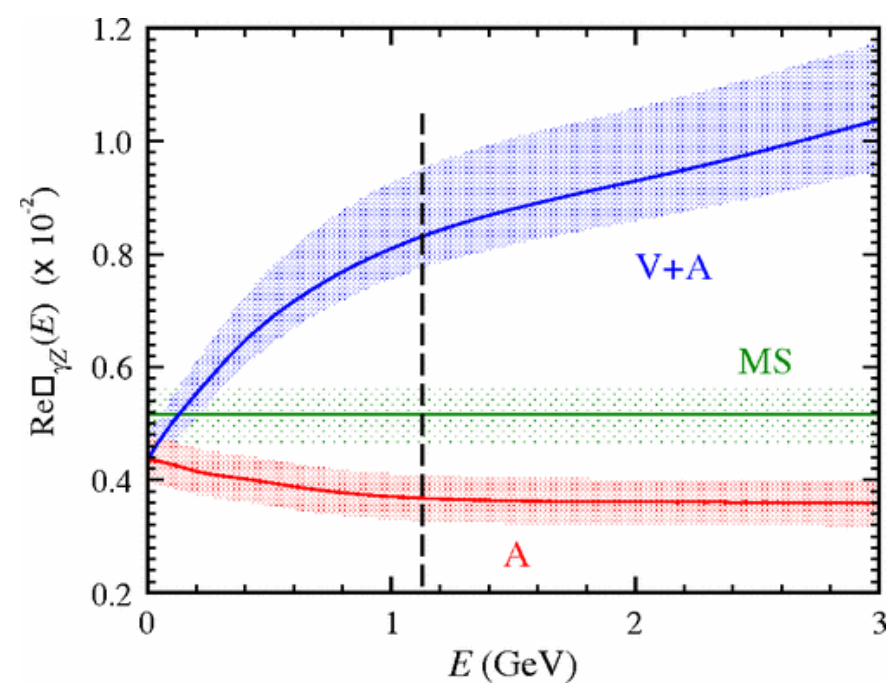

Figure 1.5: The energy dependence of the $\square_{\gamma Z}$. The total axial-hadron correction is shown in red. The blue band, labelled $V+A$, is the sum of both axial- and vector-hadron contributions. The dashed line gives the energy of the $Q_{\text {weak }}$ experiment [26].

The axial-proton piece is highly energy dependent, and approximately $8 \%$ at $Q_{\text {weak }}$ 's energy. Over the past several years, a large theoretical effort has focused on this particular radiative contribution. Calculations seem to agree on the central value, but its uncertainty is disputed. For the analysis discussed in Chapter 7, the latest results are used for the vector contribution [27],

$$
\mathfrak{R e} \square{ }_{\gamma Z}^{V}=(5.4 \pm 0.4) \times 10^{-3} .
$$

Table 1.2 summarizes the sizes of the relevant electroweak radiative corrections for extracting $\sin ^{2} \theta_{W}$ from a measurement of the proton's weak charge. 


\begin{tabular}{|l|c|c|}
\hline Correction & Value & Reference \\
\hline $\sin ^{2} \hat{\theta}_{W}\left(M_{Z}\right)$ & 0.23126 & {$[20]$} \\
\hline$\rho_{N C}$ & 1.00833 & {$[28]$} \\
\hline$\Delta_{e}$ & -0.00116 & {$[25]$} \\
\hline$\Delta_{e}^{\prime}$ & -0.00142 & {$[25]$} \\
\hline$\square_{W W}$ & 0.01832 & {$[25]$} \\
\hline$\square_{Z Z}$ & 0.00193 & {$[25]$} \\
\hline$\square_{\gamma Z}^{A}$ & 0.0037 & {$[26]$} \\
\hline$\square_{\gamma Z}^{V}$ & 0.0054 & {$[27]$} \\
\hline
\end{tabular}

Table 1.2: Summary of electroweak radiative corrections for $Q_{\text {weak }}$. 


\section{Chapter 2}

\section{Parity-Violating Electron Scattering and the $Q_{\text {weak }}$ Experiment}

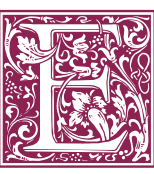

LECTRON SCATTERING HAS a rich, successful experimental history in describing nuclear structure. Experiments over the last 50 years have specialized in

mapping out the distribution of electric charge and magnetization of nuclei; improvements in experimental techniques and technologies now allow for electron scattering experiments to probe even higher order processes. In particular, the technique of using parity-violating electron scattering has allowed exploration of strangeness contributions in the nucleon, as well as critical tests of the Standard Model.

This chapter focuses on electron scattering as a probe of electroweak interactions, and in particular parity-violating electron scattering. A brief description of electroweak form factors will be presented. Since this thesis focuses on the contribution of the $Q_{\text {weak }}$ target's thin aluminum walls, electroweak form factors of neutron rich nuclei will also be discussed.

\subsection{Electromagnetic form factors}

It's well known from field theory that in every electroweak interaction, all possible processes contribute to the scattering amplitude. Therefore, in each electromagnetic interaction, at tree level, there is a small contribution from $Z^{0}$ exchange. This process is heavily suppressed due to the large $Z^{0}$ mass, but it is possible to access this, either through measuring atomic energy level transitions or parity-violating electron scattering.

To fully understand electron-nucleon scattering, both the electron and nucleon exchange vertices must be known. At tree level, electroweak interactions involve exchange 
of either a photon or $Z^{0}$ boson (Figure 2.1). The electron vertices are related to the electroweak currents determined in equations (1.10) and (1.34):

$$
\begin{aligned}
& J_{e, \gamma}^{\mu}=-e \bar{u}_{e} \gamma^{\mu} u_{e} \\
& J_{Z, \gamma}^{\mu}=-e \bar{u}_{e} \gamma^{\mu}\left(g_{V}-g_{A} \gamma_{5}\right) u_{e}
\end{aligned}
$$

where $u_{e}$ and $\bar{u}_{e}$ are the incoming and outgoing electron spinors, respectively.

Nucleon currents are more complicated because they are composite particles, with complex internal structure. To account for this, we can write the nucleon vertex in terms of two vector form factors, ${ }^{1} F_{1}\left(Q^{2}\right)$ and $F_{2}\left(Q^{2}\right)$, respectively, as

$$
\begin{aligned}
& J_{\gamma}^{\mu}=\bar{\psi}\left[F_{1}^{\gamma}\left(Q^{2}\right) \gamma^{\mu}+F_{2}^{\gamma}\left(Q^{2}\right) \frac{i \sigma^{\mu \nu} q_{\nu}}{2 M}\right] \psi \\
& J_{Z}^{\mu}=\bar{\psi}\left[F_{1}^{Z}\left(Q^{2}\right) \gamma^{\mu}+F_{2}^{Z}\left(Q^{2}\right) \frac{i \sigma^{\mu \nu} q_{\nu}}{2 M}+G_{A}^{Z}\left(Q^{2}\right) \gamma^{\mu} \gamma_{5}\right] \psi,
\end{aligned}
$$

where $M$ is the nucleon's mass and $G_{A}^{Z}$ is the axial current. ${ }^{2}$ This is the most general, Lorentz invariant expression for a nucleon vertex. Generally it's conceptually more profitable to rephrase equations (2.3) in terms of linear combinations of $F_{1}$ and $F_{2}$, as

$$
\begin{aligned}
& G_{E}^{\gamma, Z}=F_{1}^{\gamma, Z}-\tau F_{2}^{\gamma, Z} \\
& G_{M}^{\gamma, Z}=F_{1}^{\gamma, Z}+F_{2}^{\gamma, Z}
\end{aligned}
$$

where $\tau$ is a kinematic factor known as the reduced proton mass: $\tau=Q^{2} / 4 M^{2} . G_{E}$ and $G_{M}$ are known as the Sachs form factors. The Fourier transform of $G_{E}$ and $G_{M}$ give the spatial electric charge and magnetization distributions of the nucleon in the Breit frame (zero energy transfer). In the static limit $\left(Q^{2} \rightarrow 0\right)$ these approach the electric charge and magnetic moment of the proton $\left(G_{E}^{p}(0)=1\right.$ and $\left.G_{M}^{p}(0)=\mu_{p}=2.79\right)$.

\footnotetext{
${ }^{1}$ Also known as the Pauli and Dirac form factors.

${ }^{2}$ To simplify notation going forward, the explicit $Q^{2}$ dependence of the form factors is assumed.
}
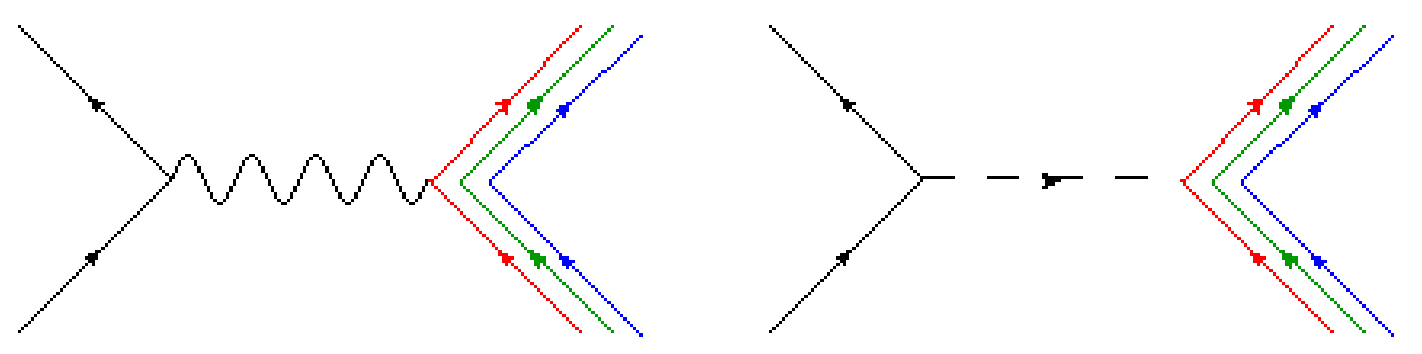

Figure 2.1: (Left) Photon exchange between $e$ and $p$. (Right) The competing process, $Z^{0}$ exchange between $e$ and $p$. 
Furthermore, these are also related to the charge radii:

$$
\begin{aligned}
& <r_{E}^{2}>=-\left.6 \frac{d G_{E}^{p, \gamma}\left(Q^{2}\right)}{d Q^{2}}\right|_{Q^{2} \rightarrow 0} \\
& <r_{M}^{2}>=-\left.6 \frac{d G_{M}^{p, \gamma}\left(Q^{2}\right)}{d Q^{2}}\right|_{Q^{2} \rightarrow 0} .
\end{aligned}
$$

The electromagnetic form factors have been studied over a wide range of kinematic regimes. At low- $Q^{2}$, the proton and neutron electric and magnetic form factors are found to follow a dipole form,

$$
G_{D}=\left(1+\frac{Q^{2}}{0.71 \mathrm{GeV}^{2}}\right)^{-2}
$$

The neutron form factors are particularly challenging to measure from lack of a pure neutron target. However, they can be determined via electron-deuteron scattering by subtracting the proton contribution. These form factors are well parameterized by the Galster fit as [29]

$$
\begin{aligned}
G_{E}^{p, \gamma} & =G_{D}, \\
G_{M}^{p, \gamma} & =\mu_{p} G_{D}, \\
G_{E}^{n, \gamma} & =G_{D}, \\
G_{M}^{n, \gamma} & =\frac{-\mu_{n} \tau G_{D}}{1+5.6 \tau},
\end{aligned}
$$

where $\mu_{n}$ is the neutron's magnetic moment. A summary of nucleon electromagnetic form factors over a wide kinematic regime is found in [30]. Low- $Q^{2}$ results can be found in the literature, $[31,32,33]$, although there are some disagreements in the implementation of Coulomb corrections to [33] (e.g., see [34]).

\subsubsection{Neutral weak form factors}

Electron-nucleon neutral weak interactions (exchange of a $Z^{0}$ ) are parameterized similarly to electromagnetic interactions, namely through vector and axial form factors, but are less well known. In general, the nucleon form factors can be written as a sum of the individual quark contributions weighted by their respective electric or weak charges. For the proton, the electromagnetic form factors can be written as

$$
\begin{aligned}
& F_{1}^{\gamma}=\sum_{f} Q^{f} F_{1}^{f}=\frac{2}{3} F_{1}^{u}-\frac{1}{3} F_{1}^{d}-\frac{1}{3} F_{1}^{s} \\
& F_{2}^{\gamma}=\sum_{f} Q^{f} F_{2}^{f}=\frac{2}{3} F_{2}^{u}-\frac{1}{3} F_{2}^{d}-\frac{1}{3} F_{2}^{s},
\end{aligned}
$$

where $u, d$, and $s$ refer to the up, down, and strange quarks, respectively. Heavier quark flavors are safely neglected in this approximation from theory [35]. The corresponding 
neutral weak form factors for the proton are defined similarly,

$$
\begin{aligned}
F_{1,2}^{Z} & =\left(1-\frac{8}{3} \sin ^{2} \theta_{W}\right) F_{1,2}^{u}+\left(-1+\frac{4}{3} \sin ^{2} \theta_{W}\right)\left(F_{1,2}^{d}+F_{1,2}^{s}\right), \\
G_{A}^{Z} & =-G_{A}^{u}+G_{A}^{d}+G_{A}^{s},
\end{aligned}
$$

where $G_{A}^{Z}$ is the neutral weak axial form factor. From these, we can now define the neutral-weak Sachs form factors, as in equation (2.4). Assuming isospin symmetry and noting that transforming protons to neutrons $(p \rightarrow n)$ is equivalent to interchanging up and down quarks $(\mathrm{u} \leftrightarrow \mathrm{d})$ allows additional relations. Taking linear combinations of equations (2.8), and combining them with the corresponding neutron versions allow the proton's neutral-weak form factors to be expressed in terms of the electromagnetic form factors as

$$
\begin{aligned}
G_{E, M}^{p, Z} & =\left(1-4 \sin ^{2} \theta_{W}\right) G_{E, M}^{p, \gamma}+(-1) G_{E, M}^{p, \gamma}+(-1) G_{E, M}^{s} \\
& =Q_{W}^{p} G_{E, M}^{p, \gamma}+Q_{W}^{n} G_{E, M}^{p, \gamma}+Q_{W}^{s} G_{E, M}^{s},
\end{aligned}
$$

where $Q_{W}^{p}$ and $Q_{W}^{n}$ are the weak charges of the proton and neutron, respectively. $G_{E, M}^{s}$ is the nucleon strange form factor with weak charge $Q_{W}^{s}$ (the strange contribution is identical for protons and neutrons). The numerical values of the weak charges (e.g., $\left.Q_{W}^{p}=1-4 \sin ^{2} \theta_{W}\right)$ can be determined from substituting the appropriate values from Table 1.1 into equation (1.36).

Considerable effort over the past two decades has focused on probing strange quark contributions in the nucleon. In the static limit, the net strangeness in the nucleon is of course zero $\left(G_{E}^{s}(0)=0\right)$ because no valence strange quarks are present. The strange magnetic moment may be non-zero $\left(\mu_{s} \equiv G_{M}^{s}(0)\right)$, however. The mean squared strange radius is generally non-zero as well due to quantum fluctuations $\left\langle r_{s}^{2}\right\rangle \equiv-\left.6\left(d G_{e} / d Q^{2}\right)\right|_{Q^{2}=0}$. The most recent results, from the HAPPEX-III collaboration at Jefferson Laboratory, suggest zero net strangeness contribution at low- $Q^{2}$ [36]. The total net strangeness is shown in Figure 2.2, and given as $G_{E}^{s}+\eta G_{M}^{s}$, where $\eta=\tau G_{M}^{p} /\left(\epsilon G_{E}^{p}\right) . \tau$ is the reduced mass previously defined, and $\epsilon=\left[1+2(1+\tau) \tan ^{2} \theta / 2\right]^{-1}$.

Neglecting strange quark effects and radiative corrections, the neutral-weak axial form factor can be expressed using a dipole form,

$$
G_{A}\left(Q^{2}\right)=G_{A}(0)\left(\frac{1}{1+Q^{2} / M_{A}^{2}}\right),
$$

where $G_{A}\left(Q^{2}=0\right)=-1.2701 \pm 0.0025$ and $M_{A}^{2}=1.00 \pm 0.04(\mathrm{GeV} / \mathrm{c})^{2}[37,38]$. Ignoring $S U(3)$ singlet components, the axial term can be split into isovector and isoscalar components. The isoscalar term is well constrained, but the isovector term involves 


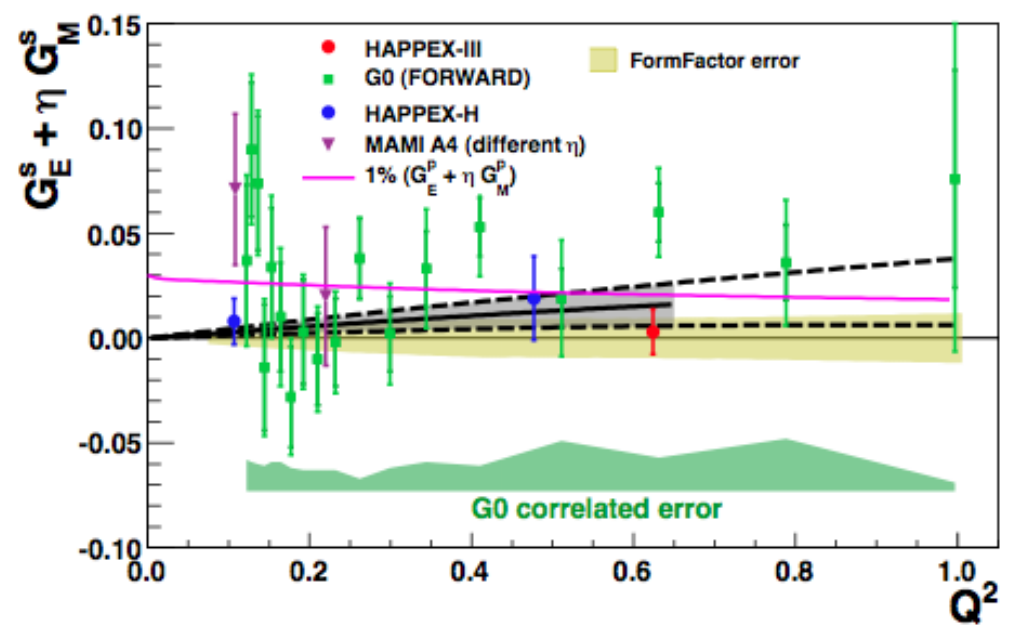

Figure 2.2: Global fit of strange form factor data at low- $Q^{2}[36]$.

large radiative corrections from many-quark effects at the proton vertex [39]. Fortunately, this contribution is generally suppressed at $Q_{\text {weak }}$ kinematics (i.e., at forward angles). The nucleon strange form factors and isovector axial form factor generally can be constrained by fitting to the world's parity-violating data set [40].

Radiative corrections to the weak nuclear form factors enter as additive factors to equations (2.12),

$$
\begin{aligned}
& G_{E, M}^{p, Z}=\left(1-4 \sin ^{2} \theta_{W}\right)\left(1+R_{V}^{p}\right) G_{E, M}^{p, \gamma}-\left(1+R_{V}^{n}\right) G_{E, M}^{p, \gamma}-G_{E, M}^{s} \\
& G_{E, M}^{n, Z}=\left(1-4 \sin ^{2} \theta_{W}\right)\left(1+R_{V}^{n}\right) G_{E, M}^{p, \gamma}-\left(1+R_{V}^{p}\right) G_{E, M}^{p, \gamma}-G_{E, M}^{s}
\end{aligned}
$$

where $R_{V}^{p}=-0.0520$ and $R_{V}^{n}=-0.0123$. The theoretical uncertainties of these corrections are less than one percent [37].

\subsection{Cross sections for $e+p$ and $e+A l$ scattering}

The $Q_{\text {weak }}$ experiment uses elastic electric-proton scattering to determine the proton's weak charge. The cross section for unpolarized elastic ep scattering in the laboratory frame can be written as a modification of the scattering from a point particle (Mott scattering), as

$$
\frac{d \sigma}{d \Omega}=\left(\frac{d \sigma}{d \Omega}\right)_{M o t t}\left|F\left(Q^{2}\right)\right|^{2}
$$

where $F\left(Q^{2}\right)$ is a general form factor describing the protons internal structure. The Mott cross section, ignoring recoil, is

$$
\left(\frac{d \sigma}{d \Omega}\right)_{M o t t}=\frac{\alpha^{2} \cos ^{2} \theta / 2}{4 E^{2} \sin ^{2} \theta / 2}
$$


$E$ is the incident beam energy, $\theta$ the scattering angle, and $\alpha$ is the fine structure constant. Expressing the scattering cross section in terms of the Sachs electromagnetic form factors (equations (2.4)) results in the Rosenbluth formula,

$$
\left(\frac{d \sigma}{d \Omega}\right)_{l a b}=\left(\frac{d \sigma}{d \Omega}\right)_{M o t t} \frac{E^{\prime}}{E}\left(\frac{\left(G_{E}^{p}\right)^{2}+\tau\left(G_{M}^{p}\right)^{2}}{1+\tau}+2 \tau\left(G_{M}^{p}\right)^{2} \tan ^{2} \frac{\theta}{2}\right)
$$

where $\theta$ is the lab scattering angle, $\tau$ is the reduced proton mass, and $\left(E^{\prime} / E\right)$ is the ratio of outgoing to incoming electron energy, which accounts for target recoil.

Of principal interest to this thesis is the contribution from the $Q_{\text {weak }}$ target's aluminum walls. This contribution is the primary subject of Chapters 5 and 6 . Therefore, the cross section for elastic electron-aluminum scattering is of particular interest. In the Born approximation, the cross section for elastic electron-aluminum scattering can be expressed by (2.16), with a simple form factor expansion [41]

$$
\left|F\left(q^{2}\right)\right|^{2}=Z^{2}\left|F_{0}\left(q^{2}\right)\right|^{2}+\frac{q^{4}}{180} \frac{(J+1)(2 J+3)}{J(2 J-1)} \mathcal{Q}^{2}\left|F_{2}\left(q^{2}\right)\right|^{2},
$$

where $q^{2}$ is the momentum transfer ${ }^{3}, \mathcal{Q}$ is the nuclear electric quadrupole moment, $J$ is the nuclear spin $\left(J=5 / 2\right.$ for $\left.{ }^{27} \mathrm{Al}\right)$, and $Z$ the atomic number. Equation (2.19) ignores the electron mass and nuclear recoil. It also neglects the magnetic terms, which are suppressed at $Q_{\text {weak }}$ 's forward angles.

A full derivation of the cross section is presented in [42], which accounts for finite proton size and additional correction factors. The largest correction, responsible for a large energy dependence in the form factor, is given by Coulomb corrections. Figure 2.3 shows the comparison of this calculation to measured data.

\subsection{Parity-violating asymmetries and electron scattering}

Although the dominant exchange in elastic electron scattering is purely electromagnetic (photon exchange), it is possible to directly access the neutral-weak interaction via parity violation. Recall from Section 1.2.1 that a parity transformation is an inversion of spatial coordinates, and that a massive particle's handedness is related to its helicity, the alignment of a particle's spin vector with its direction-of-momentum $(h \equiv \mathbf{s} \cdot \hat{\mathbf{p}})$.

Helicity is a pseudo-scalar, and is an eigenstate of parity with eigenvalue -1 . Therefore given a longitudinally polarized electron beam scattering off an unpolarized target, switching the longitudinal beam polarization (from aligned with its momentum to antialigned, or vice-versa) is equivalent to a parity transformation.

\footnotetext{
${ }^{3}$ It is written this way to remind the reader that recoil is ignored.
} 


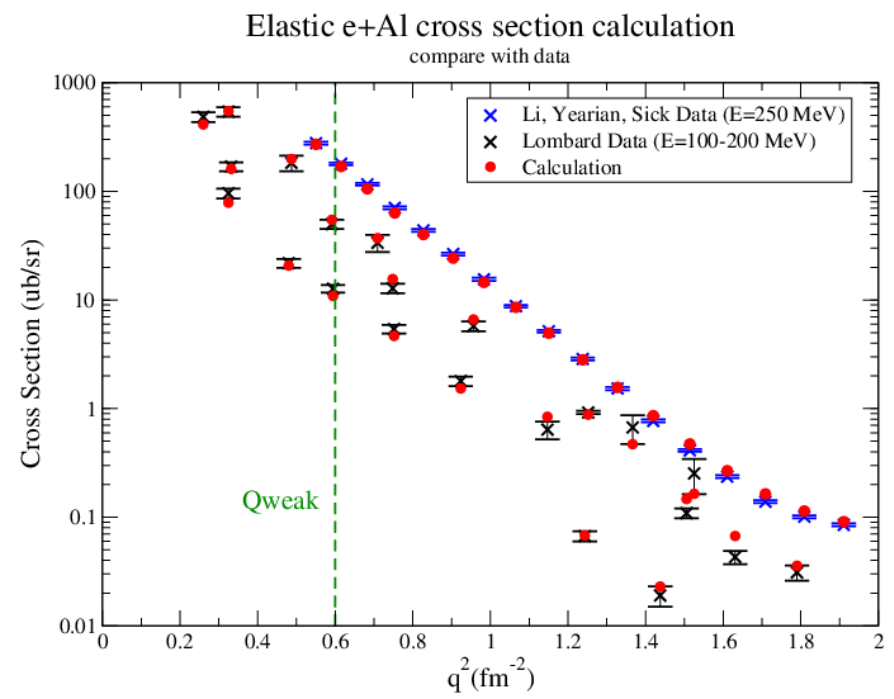

Figure 2.3: Calculated $e+A l$ cross section versus momentum-transfer (ignoring nuclear recoil). The calculation is from [41], and compared to data from [43, 44]. Reproduced from [42].

Recall that a scattering cross section is proportional to the square of the total scattering amplitude, $\mathcal{M}_{\text {tot }}$,

$$
\sigma \propto\left|\mathcal{M}_{\text {tot }}\right|^{2},
$$

where the $\mathcal{M}_{\text {tot }}=\mathcal{M}_{\gamma}+\mathcal{M}_{Z}$. We can define the parity-violating asymmetry as the quotient of the average difference and average sum between the cross section for right (left) handed electrons, $\sigma_{R}\left(\sigma_{L}\right)$,

$$
A_{P V} \equiv \frac{\sigma_{R}-\sigma_{L}}{\sigma_{R}+\sigma_{L}} .
$$

Recall that the $Z^{0}$ vertex has a parity-violating piece (equation (1.34)) while the $\gamma$ vertex is purely vectorial (equation (1.10)). Therefore the total scattering amplitudes for right and left handed particles differ by the sign of the $Z^{0}$ terms:

$$
\begin{aligned}
& \mathcal{M}_{R}=\mathcal{M}_{\gamma}+\mathcal{M}_{Z} \\
& \mathcal{M}_{L}=\mathcal{M}_{\gamma}-\mathcal{M}_{Z} .
\end{aligned}
$$

Substituting (2.22) into (2.21), one finds complete cancellation except for the $\gamma-Z$ interference cross-term,

$$
A_{P V} \propto \frac{4 \mathcal{M}_{\gamma} \mathcal{M}_{Z}}{2\left(\mathcal{M}_{\gamma}+\mathcal{M}_{Z}\right)} \approx \frac{2 \mathcal{M}_{Z}}{M_{\gamma}} \approx 10^{-7},
$$

where we have used the fact the $\left|\mathcal{M}_{Z}\right| \ll\left|\mathcal{M}_{\gamma}\right|$.

For elastic electron-proton scattering, we can also write $A_{P V}$ in terms of the electroweak and axial form factors as [35, 37]

$$
A_{P V}^{e p}=\left[\frac{G_{F} Q^{2}}{4 \pi \alpha \sqrt{2}}\right] \cdot\left[\frac{\epsilon G_{E}^{p, \gamma} G_{E}^{p, Z}+\tau G_{M}^{p, \gamma} G_{M}^{p, Z}-\left(1-4 \sin ^{2} \theta_{W}\right) \epsilon^{\prime} G_{M}^{p, \gamma} G_{A}^{Z}}{\epsilon\left(G_{E}^{p, \gamma}\right)^{2}+\tau\left(G_{M}^{p, \gamma}\right)^{2}}\right],
$$


where $G_{F}$ is the Fermi constant, and $\tau, \epsilon$, and $\epsilon^{\prime}$ are the previously defined kinematical factors,

$$
\tau=\frac{Q^{2}}{4 M_{p}^{2}}, \quad \epsilon=\left[1+2(1+\tau) \tan ^{2} \frac{\theta}{2}\right], \quad \epsilon^{\prime}=\sqrt{\tau(1+\tau)\left(1-\epsilon^{2}\right)} .
$$

Note that the axial (third) term in equation (2.24) arises from the electron current; $-\left(1-4 \sin ^{2} \theta_{W}\right)$ is actually the weak coupling of the electron here.

By substituting (2.12) into (2.24) and grouping proton, neutron, strange, and axial terms, we can then write $(2.24)$ as a sum of three contributions,

$$
A_{P V}^{e p}=\left[\frac{G_{F} Q^{2}}{4 \pi \alpha \sqrt{2}}\right] \cdot\left[Q_{W}^{p}+A_{\text {hadronic }}+A_{\text {axial }}\right]
$$

where

$$
\begin{aligned}
Q_{W}^{p} & =1-4 \sin ^{2} \theta_{W}, \\
A_{\text {hadronic }} & =\frac{\left(Q_{W}^{n}+Q_{W}^{s}\right)\left(\epsilon G_{E}^{p, \gamma} G_{E}^{n, \gamma}+\tau G_{M}^{p, \gamma} G_{M}^{n, \gamma}\right)}{\epsilon\left(G_{E}^{p, \gamma}\right)^{2}+\tau\left(G_{M}^{p, \gamma}\right)^{2}}, \text { and } \\
A_{\text {axial }} & =\frac{-\left(1-4 \sin ^{2} \theta_{W}\right) \epsilon^{\prime} G_{M}^{p, \gamma} G_{A}^{Z}}{\epsilon\left(G_{E}^{p, \gamma}\right)^{2}+\tau\left(G_{M}^{p, \gamma}\right)^{2}} .
\end{aligned}
$$

In the limit of forward angles $(\theta \rightarrow 0)$ and zero-momentum transfer $\left(Q^{2} \rightarrow 0\right)$, the strange contribution vanishes and the kinematic variables $\epsilon$ and $\epsilon^{\prime}$ approach 1 and 0 , respectively $\left(\epsilon \rightarrow 1, \epsilon^{\prime} \rightarrow 0\right)$. Under these conditions, the hadronic and axial components are heavily suppressed. The tree-level asymmetry can now be re-written in terms of a general hadronic form factor, $B\left(Q^{2}, \theta\right)$,

$$
A_{P V}^{e p}=\frac{G_{F} Q^{2}}{4 \pi \alpha \sqrt{2}}\left[Q_{W}^{p}+Q^{2} B\left(Q^{2}, \theta\right)\right] .
$$

The leading order term is the proton's weak charge, while the next-to-leading term is the first place hadronic structure enters. At $Q_{\text {weak }}$ kinematics, this term contributes approximately $\sim 30 \%$ to the measured asymmetry, and its uncertainty is dominated by contributions from the mean-square strange radius and magnetic moment.

Often it is more convenient to divide out the leading $Q^{2}$ dependence of (2.30). This is called the reduced asymmetry, and is advantageous because when written this way $Q_{W}^{p}$ is the intercept, and $B$ is a slope-like term,

$$
\frac{A_{P V}}{A_{0}}=Q_{W}^{p}+Q^{2} B\left(Q^{2}, \theta\right)
$$

where $A_{0}=-G_{F} Q^{2} / 4 \pi \alpha \sqrt{2}$, and higher order terms in the expansion are neglected. Written in this form, the world's parity-violating data set can be fit and extrapolated to $Q^{2}=0$, leading to a determination of the proton's weak charge. Before 2012, 
parity-violating electron scattering data at low- $Q^{2}$ was lacking, leading to substantial uncertainty in such an extrapolation [45]. The subject of this thesis, and in particular Section 2.4, is $Q_{\text {weak }}$, a low- $Q^{2}$ determination taking place at Jefferson Lab, which will reduce the uncertainty in such an extrapolation.

In addition to the parity-violating asymmetry in hydrogen, $Q_{\text {weak }}$ also spent substantial time measuring the parity-violating asymmetry of aluminum to constrain the background of the target's aluminum walls. An estimate of this asymmetry, good to about $10 \%$, is given by [46],

$$
A_{P V}\left({ }^{27} A l\right)=\frac{-G_{F} Q^{2}}{4 \pi \alpha \sqrt{2}}\left[Q_{W}^{p}+Q_{W}^{n}\left(\frac{N}{Z}\right)\right]
$$

where $Z$ and $N$ are the number of protons and neutrons in the aluminum nucleus. This calculation assumes dominance of the charge monopole, which is reasonable at $Q_{\text {weak }}$ kinematics, as higher-order effects only contribute approximately $30 \%$ [42]. In addition, proton and neutron distributions, $\rho_{p}$ and $\rho_{n}$ respectively, are assumed to differ only by a normalization,

$$
\rho_{n}(x) / N=\rho_{p}(x) / Z .
$$

This assumption is reasonable for $Q_{\text {weak }}$ as the average $Q^{2}$ is well below the cross section diffraction minimum, where isospin symmetry violating effects may be large. In general, isospin symmetry violation is approximately $1 \%$ at $Q_{\text {weak }}$ kinematics. More modern models have recently been published [47], which will be discussed in Chapter 7.3 .

\section{$2.4 Q_{\text {weak }}$ introduction}

The $Q_{\text {weak }}$ experiment made a precision measurement of the parity-violating asymmetry in elastic $\vec{e} p$ scattering at low- $Q^{2}\left(Q^{2}=0.025 \mathrm{GeV}^{2}\right)$ [48]. As seen in Section 2.3, if hadronic structure effects are well-constrained, a measurement of $A_{P V}^{e p}$ allows extraction of the proton's weak charge, $Q_{W}^{p}$. Furthermore, this allows direct access to a precise determination of $\sin ^{2} \theta_{W}$ at low- $Q^{2}$.

These recently published results are from an initial commissioning run (Run 0), which contains only $4 \%$ of the total data set. This thesis focuses on two critical improvements over those initial results: the high-precision determination of the electron beam polarization and removal of background contributions originating from scattering off of the target's aluminum windows. These improvements allow a more robust extraction, and directly enable tighter constraints on possible Standard Model extensions, as discussed at the end of this section. 
Figure 2.4 shows a global fit of the reduced asymmetries (equation (2.31)) of the world's data at moderate to low- $\mathrm{Q}^{2}$. Previous experiments were designed to probe the hadronic, and in particular strange, components of the nucleons (HAPPEX [49, 36], SAMPLE [50], PVA4 [51], G0 forward [52], G0 backward [53]). In order to plot the two-dimensional global fit $\left(Q^{2}, \theta\right)$ in a single dimension, the angular dependence of the strange and axial form-factors was removed from the measured asymmetries. The points were all individually corrected for the $\square_{\gamma Z}$ energy dependence [54] and for the $Q^{2}$ dependence [55], where appropriate. The red point is the current, $Q_{\text {weak }} 4 \%$ result, while the black arrow is the Standard Model prediction at $Q^{2}=0$.

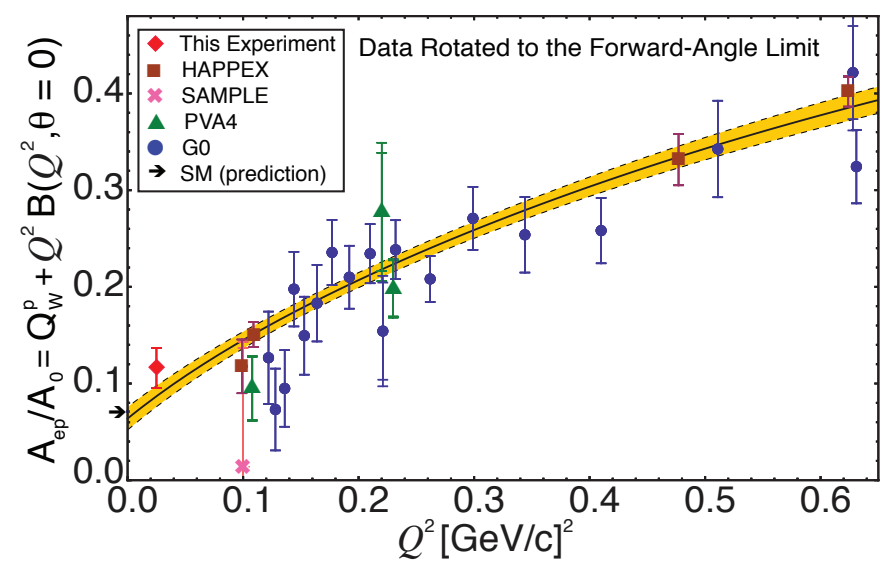

Figure 2.4: Global fit of world's parity-violating electron scattering data. The red diamond at low- $Q^{2}$ is the initial $Q_{\text {weak }}$ point, comprising only $4 \%$ of the total dataset. From [48].

Note that $Q_{\text {weak }}$ ran at substantially lower $Q^{2}$ than the other experiments. This has several important ramifications for the extrapolation to $Q^{2}=0$. First, $Q_{\text {weak }}$ is relatively insensitive to any proton internal structure compared to previous experiments. Furthermore, it is unlikely that additional nuclear structure effects exist using even longer-range probes, and the relatively short extrapolation distance also increases confidence in the extrapolation procedure. Additionally, even with its modest significance, the current result already provides noticeable pull, and shifts the fit result approximately $1 \sigma$. For these reasons, the $Q_{\text {weak }}$ experiment claims the first direct determination of the proton's weak charge.

As discussed in Section 1.3.4, the weak charge of the proton is the coherent sum of the individual quark weak vector couplings, $C_{1 u}$ (up quark) and $C_{1 d}$ (down quark). Measurements of parity-violation in atomic transitions provide complementary measurements proportional to a different combination of quark couplings $\left(Q_{W}\left({ }^{133} \mathrm{Cs}\right)=\right.$ $C_{1 u}+1.12 C_{1 d}$ from equation (1.39)). Combining these results allows extraction of 
the individual quark-vector couplings. The neutron's weak charge, $Q_{W}^{n}$, may then be determined.

Figure 2.5 shows the current constraints on the isoscalar $\left(C_{1 u}+C_{1 d}\right)$ and isovector $\left(C_{1 u}-C_{1 d}\right)$ combinations of the quark vector couplings [48]. The green band comes from a atomic parity-violating measurement of ${ }^{133} \mathrm{Cs}$ [56]. The blue ellipse is the world's current parity-violating data set with $Q^{2}<0.63$, including $Q_{\text {weak }}$. The (smaller) red ellipse is the combined fit, and black dot the Standard Model prediction. Inner (outer) ellipses are $68 \%(95 \%)$ confidence level. The Standard Model prediction in the $\overline{M S}$ scheme is shown as a projection on the weak mixing angle axis (black dot). The current findings are in excellent agreement with the Standard Model predictions.

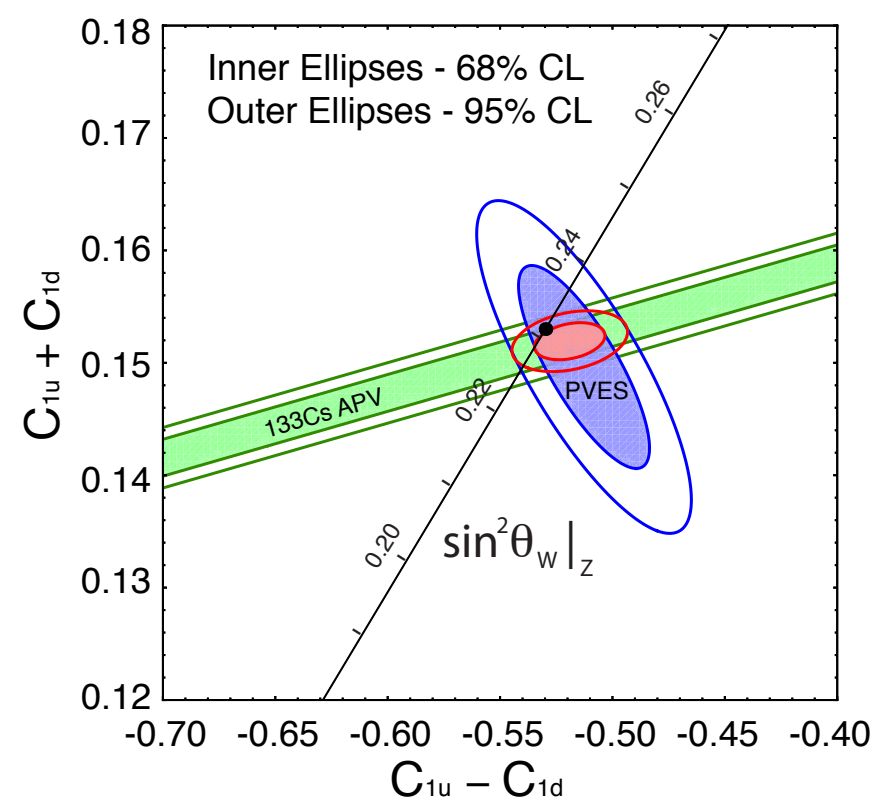

Figure 2.5: Constraints on the weak vector quark couplings $C_{1 u}$ and $C_{1 d}$. From [48].

Determination of $Q_{\text {weak }}$ allows for an extraction of $\sin ^{2} \theta_{W}$, once radiative effects are accounted for (see Section 1.4). Figure 2.6 shows the current experimental limits on the evolution of $\sin ^{2} \theta_{W}$ with $Q^{2}$, and the preliminary $Q_{\text {weak }}$ datum (labeled $Q_{W}(p)$ [48]). The Standard Model prediction is firm; the calculation's uncertainty is given by the thickness of the blue line. Measurements at the $Z^{0}$ pole fix the overall scale of $\sin ^{2} \theta_{W}$, but low- $Q^{2}$ measurements are required to describe its evolution, a key prediction of the Standard Model. Multiple, independent measurements at low- $Q$ experimentally confirm the weak mixing angle's evolution.

Several low-energy measurements are featured in Figure 2.6. The atomic parityviolating Cesium experiment measured the electric dipole transition between $6 \mathrm{~S}$ and $7 \mathrm{~S}$ states, which is forbidden by parity-selection rules [56]. A small neutral-current 


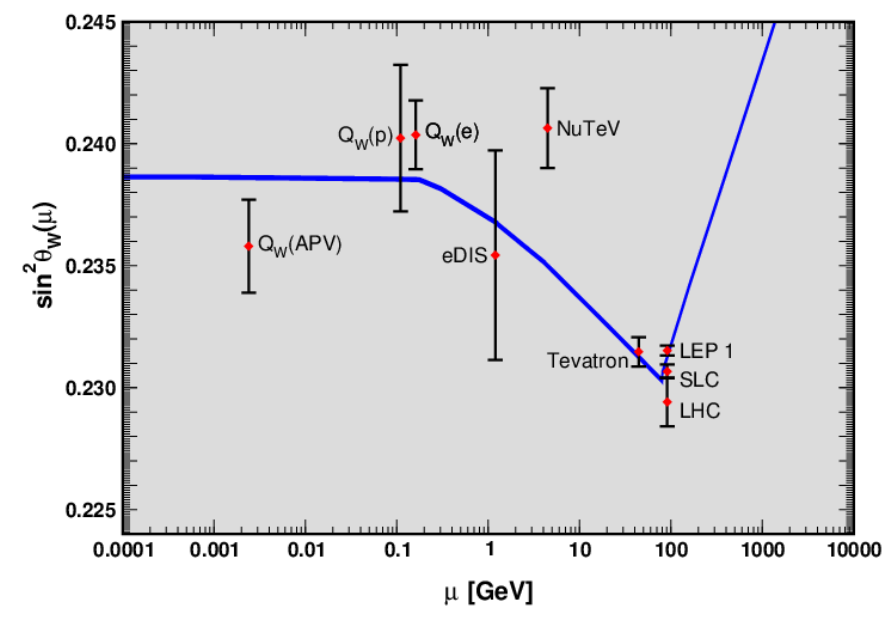

Figure 2.6: Experimental determination of the weak mixing angle vs. $Q\left(\sqrt{Q^{2}}\right)$ in the $\overline{\mathrm{MS}}$ renormalization scheme [20].

contamination $\left(Z^{0}\right.$ exchange) produces a small amount of mixing $\left(\sim 10^{-11}\right)$ between the $P$ state and $6 \mathrm{~S}$ and $7 \mathrm{~S}$ states, resulting in a parity-violating transition amplitude. This result originally reported a determination of $\sin ^{2} \theta_{W}$ at low- $Q^{2}$ to $\sim 2 \%$. Two years later, improved measurements of required quantities (e.g., the Stark vector transition polarizability) reduced the theoretical uncertainty considerably, leading to an apparent 2.5 $\sigma$ deviation from the Standard Model [57]. Considerable theoretical work has resulted in reducing this discrepancy [58], but a small deviation from the prediction remains.

The $Q_{W}(e)$ point comes from the E158 experiment performed at the Stanford Linear Accelerator, which measured the parity-violating asymmetry of electron-electron (Møller) scattering at $Q^{2}=0.026 \mathrm{GeV}^{2}$ [59]. Their results unambiguously confirmed the $\sin ^{2} \theta_{W}$ running with a $6 \sigma$ significance from the $Z^{0}$-pole, and are in moderate agreement with the Standard Model prediction. The current $Q_{\text {weak }}$ results are in agreement with both E158 and the Standard Model, but have considerable uncertainty. The final $Q_{\text {weak }}$ result will be the most precise measurement of $\sin ^{2} \theta_{W}$ at low- $Q^{2}$ to date.

Perhaps the most striking experimental result is the point from the NuTeV collaboration. This experiment, performed at FermiLab, measured deep inelastic neutrinonucleus scattering using a lead target, inferring information about the nucleons from known nuclear effects [60]. Their initial results are $3 \sigma$ away from the Standard Model, however subsequent theoretical development has shown several possible mechanisms to explain this disagreement, such as an asymmetric strange quark sea or parton-level charge symmetry violations [61]. In particular, [62] suggests that the neutron excess in the lead nucleus imposes a shift in the quark distributions (an isovector EMC effect), 
which may account for as much as two-thirds of this discrepancy. None of these corrections have been implemented, because of large theoretical uncertainties, and sadly, in the case of the isovector EMC effect, it has never been measured. Interpretation of this result is therefore difficult.

Precise measurements of $\sin ^{2} \theta_{W}$, deeply probe the fundamental structure of the Standard Mode. Therefore, they are sensitive to certain classes of potential new physics scenarios beyond the Standard Model. The significance of these low-energy experiments becomes even more evident when comparing related experiments with differing dependencies on particular models. For instance, purely leptonic interactions, such as measured in E158, are insensitive to models coupling directly to quarks; instead they would act as controls to semi-leptonic probes, such as $Q_{\text {weak }}$. Figure 2.7 compares the sensitivity between $Q_{\text {weak }}$ and E158 as an example.

Discussion of the various possible new physics models is outside the scope of this thesis, but popular ones include scaler leptoquarks (particles sharing both lepton and quark quantum numbers) [63], various $Z^{\prime}$ models from extra $U(1)$ gauge couplings $[64,65]$, and certain subclasses of supersymmetry (R-parity violating supersymmetry ${ }^{4}$ ) [66]. Concise discussions of the various models are given in [25, 61]. Figure 2.7 compares the sensitivity of E158 and $Q_{\text {weak }}$ to various models.
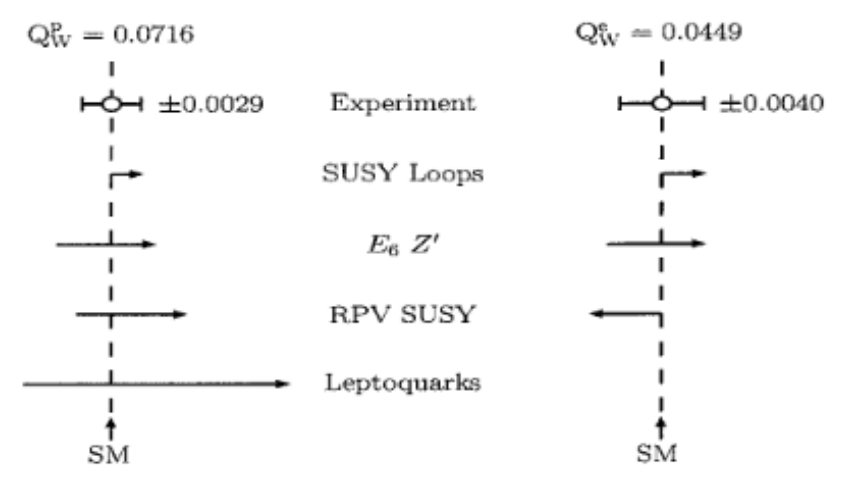

Figure 2.7: Comparison of sensitivities to various beyond the Standard Model scenarios between $Q_{\text {weak }}$ and E158 [25].

New physics may enter the Standard Model Lagrangian through the addition of a four-point contact interaction term [45],

$$
\mathcal{L}_{N P}=\frac{g^{2}}{4 \Lambda^{2}} \bar{e} \gamma_{\mu} \gamma_{5} e \sum_{q} h_{V}^{q} \bar{q} \gamma^{\mu} q,
$$

where $q$ is quark flavor, $g$ is the interaction coupling strength, $\Lambda$ the particle mass, and

\footnotetext{
${ }^{4} \mathrm{R}$-parity violation is one subset of supersymmetry, where the difference between baryon and lepton numbers is not conserved.
} 
$h_{V}^{q}$ parameterizes the interaction's isospin dependence: $h_{V}^{u}=\cos \theta_{h}$ and $h_{V}^{d}=\sin \theta_{h}$.

Addition of such contact interactions into the Lagrangian is generally model independent. To determine sensitivity, one must then choose the model parameters, $g$ and $\Lambda$. The model independent mass limit for $Q_{\text {weak }}$ is [25]

$$
\frac{\Lambda}{g} \sim \frac{1}{\sqrt{\sqrt{2} G_{F}\left|\Delta Q_{W}^{p}\right|}},
$$

where $\left|\Delta Q_{W}^{p}\right|$ is the final uncertainty on $Q_{\text {weak }}$ 's determination of $Q_{W}^{p}$. Figure 2.8 shows the mass reach in $\mathrm{TeV}$ as a function of coupling strengths for different levels of final significance in the determination of $Q_{W}^{p}$. The mass reach easily reaches into the $\mathrm{TeV}$ range for reasonable values of the coupling.

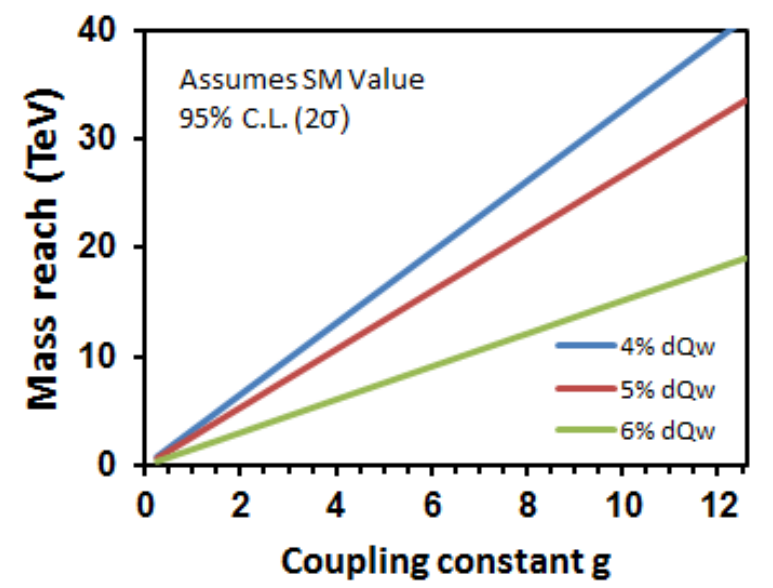

Figure 2.8: Model-independent mass reach of possible new physics versus interaction coupling strength for various $Q_{W}^{p}$ significance. Despite being a low-energy measurement, the mass reach probed is in the $\mathrm{TeV}$ range. This predicted reach is based on reference $[25]$. 


\section{Chapter 3}

\section{Experimental Setup}

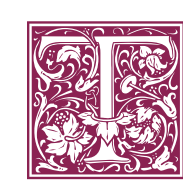

HE $Q_{\text {weak }}$ EXPERIMENT (E08-016) was performed at the Thomas Jefferson National Accelerator Facility's (JLab) Hall C with a custom built apparatus. Commissioning began in July 2010, with production running periodically over the next two years, divided into four distinct sections:

- Commissioning: Mid 2010

- Run 0: January 2011

- Run 1: February - May 2011

- Run 2: November 2011 - May 2012

Each run period has its own unique blinding factor, that offsets the measured asymmetries by up to \pm 60 parts-per-billion. This masks the true asymmetry value until the analysis is complete. Therefore, each run period is unblinded and released only when the analysis is appropriately mature for that individual period. For example, the Run 0 results only comprised $4 \%$ of the $Q_{\text {weak }}$ data set, and the results have already been published [48], while the other two run periods remain in the analysis phase.

Being a precision experiment, $Q_{\text {weak }}$ required a high-current of highly polarized electrons. It also required rapid reversal, or flip, of the beam helicity at a rate of $\approx 1$ $\mathrm{kHz}$. These requirements, among others, made Jefferson Laboratory an ideal facility for this experiment.

This chapter provides a detailed description of the experimental apparatus, beginning with the creation and transport of the "high/parity quality" electron beam, and continuing through the target apparatus, the detector arrays, background shielding, and data acquisition. Although a thorough treatment will be provided, a complete treatment is beyond the scope of this document, and can be found in the literature [67]. 


\subsection{Experimental apparatus}

$Q_{\text {weak }}$ made a precise measurement of elastic electron-proton scattering $(\vec{e} p)$. The kinematics of such a process are well understood (Figure 3.1). An electron with energy $E$, momentum $p$, and negligible mass elastically scatters off a proton of mass $M$ at rest. The final energy and momentum of the electron are given by $E^{\prime}$ and $p^{\prime}$, respectively. The energy transfer is given by $\nu=E-E^{\prime}$ and 3-momentum transfer by $\mathbf{q}=\mathbf{p}-\mathbf{p}^{\prime}$. In general, the total 4-momentum transfer, $Q^{2}$ is defined as

$$
Q^{2}=-q^{2}=-\left(\nu^{2}-\mathbf{q}^{2}\right)
$$

The momentum transfer can be empirically determined from knowledge of three quantities: the incident beam energy $(E)$, scattering angle $(\theta)$, and final electron scattering energy $\left(E^{\prime}\right)$. For elastic processes, any pair of these observables will suffice. Since $Q_{\text {weak }}$ can more precisely determine $\theta$ than $E^{\prime}$, it is advantageous to use the relation for elastic scattering in terms of $\theta$ and $E$ :

$$
Q^{2}=2 E \frac{1-\cos \theta}{1+\frac{E}{M}(1-\cos \theta)} .
$$

To suppress hadronic structure effects and inelastic processes, $Q_{\text {weak }}$ measured at low $Q^{2}$ and forward angles $\left(5.8^{\circ}<\theta<11.6^{\circ}\right)$. A list of the relevant kinematic quantities for $Q_{\text {weak }}$ is given in Table 3.1.

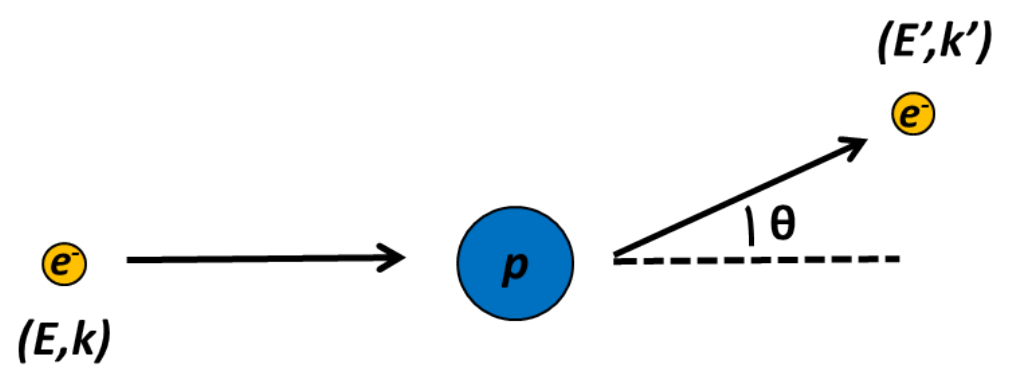

Figure 3.1: The relevant kinematics for an elastic two-body scattering process. An electron is incident with 4 -momentum $(E, k)$ and scatters off a proton at rest. The electron scatters with resultant 4 -momentum $\left(E^{\prime}, k^{\prime}\right)$.

Figure 3.2 gives an overview of the custom $Q_{\text {weak }}$ apparatus. The electron beam is $\geq 85 \%$ longitudinally polarized. Elastically scattered electrons exit the target, and a series of lead collimators selects only those events in the angular range of interest. A large toroidal magnet acts as a spectrometer, focusing them onto eight azimuthally symmetric quartz Cherenkov main detectors. Several planned periods of low-current running were used for systematic investigations. During these tracking-mode periods, 


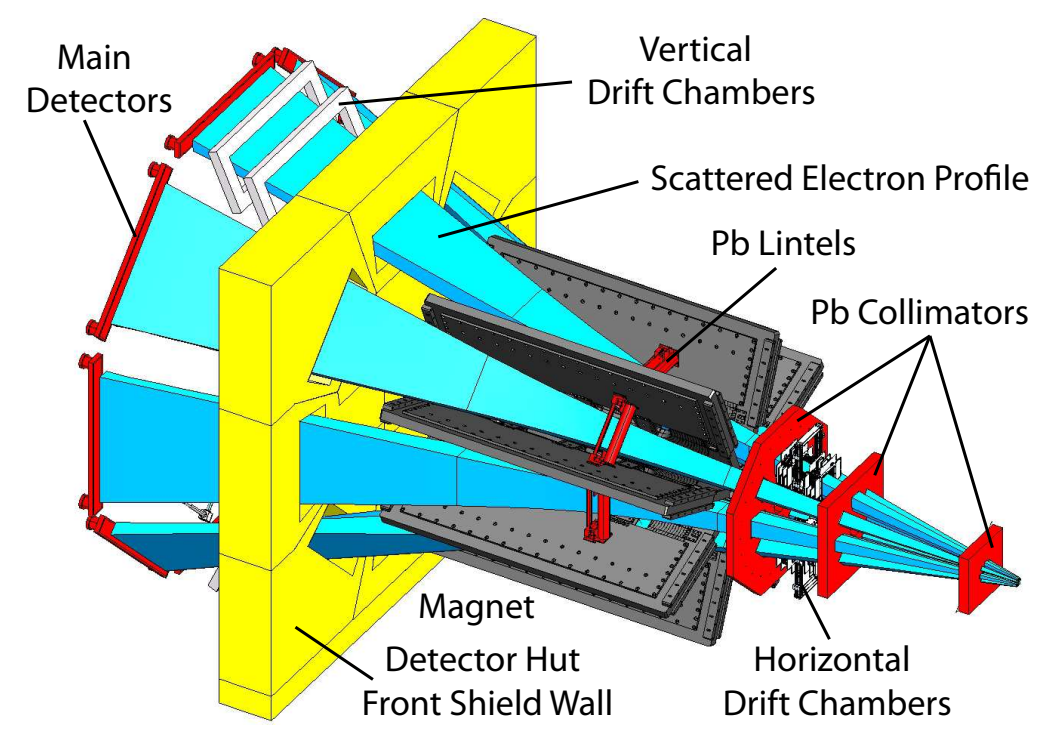

Figure 3.2: The $Q_{\text {weak }}$ experimental apparatus. The electron beam enters from the right. After exiting the target (not shown), scattered electrons progress through a series of lead collimators and a large toroidal magnet spectrometer. This spectrometer focuses the elastically scattered events onto the quartz Cherenkov main detectors. A series of plastic trigger scintillators and horizontal and vertical drift chambers are inserted periodically in low-current tracking mode, where individual scattering events are reconstructed. The blue bands show the eight scattered electron profiles defined by the collimator triplet. Figure courtesy of Valerie Gray.

a series of horizontal and vertical drift chambers and plastic trigger scintillators were inserted.

\begin{tabular}{lc}
\hline \hline Parameter & $\underline{\text { Value }}$ \\
Incident beam energy & $1.160 \mathrm{GeV}$ \\
Beam polarization & $85-90 \%$ \\
Beam current & $180 \mu A$ \\
Mean scattering angle & $7.9^{\circ}$ \\
$\theta$-acceptance & $5.8^{\circ}-11.6^{\circ}$ \\
$\phi$-acceptance & $49 \%$ of $2 \pi$ \\
Solid angle & $\Delta \Omega=43 \mathrm{msr}$ \\
Acceptance-averaged $Q^{2}$ & $\left\langle Q^{2}\right\rangle=0.025(\mathrm{GeV} / \mathrm{c})^{2}$ \\
\hline \hline
\end{tabular}

Table 3.1: Typical kinematics and operating parameters of the $Q_{\text {weak }}$ experiment.

\subsection{Beam delivery}

Parity-violation experiments place higher demands on the electron source and accelerator than typical experiments performed at JLab, in terms of the stringent control of beam parameters and high current required. In this regard, $Q_{\text {weak }}$ is considered the most demanding parity-violating experiment performed at JLab to date [68]. In partic- 
ular, $Q_{\text {weak }}$ 's requirements include precise control of beam properties for both helicity states; i.e., any helicity-dependent difference in the electron beam must be minimized. For these reasons the electron source is considered an integral part of the $Q_{\text {weak }}$ experiment.

Conceptually, generation of parity-quality beams is simple [69]: a high-powered laser producing circularly polarized light is incident on a strained gallium-arsenide (GaAs) photocathode, producing electrons via the photoelectric effect, which are then accelerated in an electrostatic field. The helicity of the electrons is transferred by the helicity of the laser light via conservation of angular momentum. The resulting electrons are then accelerated via superconducting radiofrequency (SRF) cavities. Figure 3.3 shows a diagram of the beam generation through delivery.

This section introduces the electron source, beam generation, the injector, the techniques of fast and slow helicity reversals, and the superconducting radio frequency (SRF) resonant cavities used to accelerate the electrons. Detailed information on all these systems can be found in references [67, 69, 70].

\subsubsection{Photocathode and beam generation}

Jefferson Laboratory was able to consistently deliver 80-90\% longitudinally polarized beam at high currents $(180 \mu A)$ throughout $Q_{\text {weak }}$. As an electron source, Jefferson Lab utilizes a strained gallium-arsenide (GaAs) photocathode. The band structure of GaAs

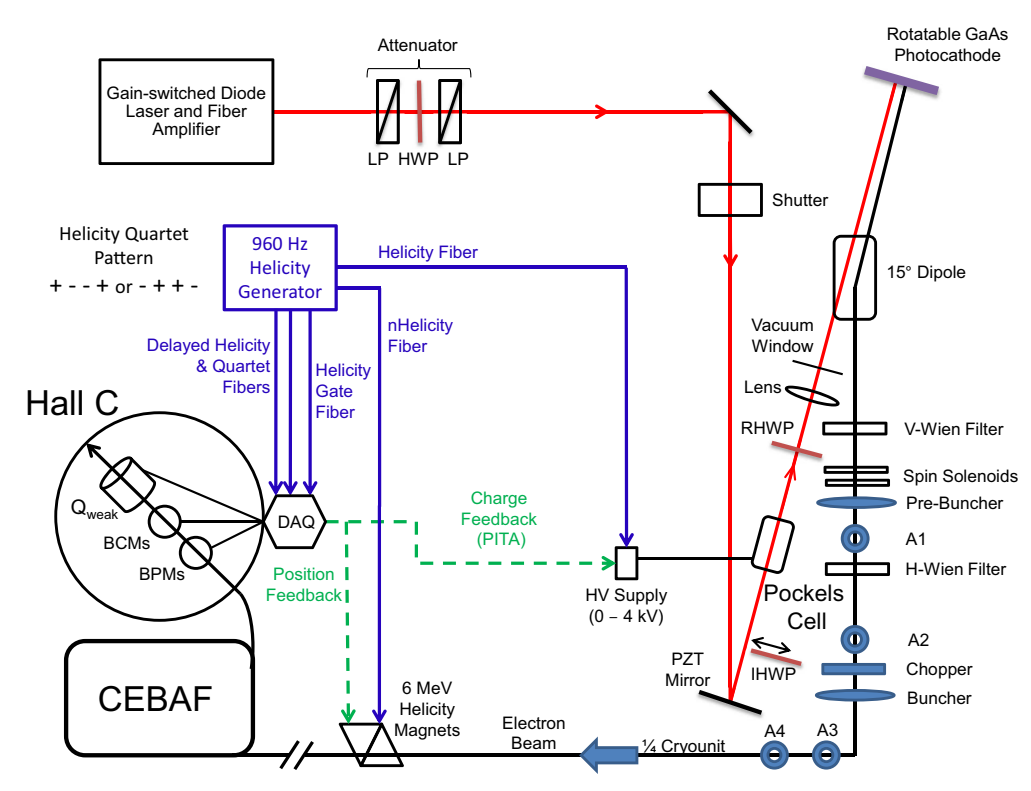

Figure 3.3: Schematic of the beam generation and delivery into the experimental halls. Note the placement of the insertable half-wave plate (IHWP), Pockels Cell, and Vertical and Horizontal Wien filters. Reproduced from [67]. 
is the primary factor enabling its use as an electron source: incident polarized (laser) light excites the electrons from the valence band to the conduction band (exciting the $p_{3 / 2}$ state to the $s_{1 / 2}$ state). Since the bands have definite angular momentum states, the excited electron will exist preferentially in a specific spin state. Standard bulk GaAs produces a maximum of $50 \%$ polarization because the $p_{3 / 2}$ states have a 4 -fold degeneracy. Inducing a strain along a certain axis of the crystal structure breaks this 4 -fold degeneracy, theoretically enabling polarizations of $100 \%$. In practice, polarizations of $75 \%$ were demonstrated in previous experiments [71]. Recently, a superlattice structure was developed by alternating layers of bulk strained GaAs. This both increased the probability of electron emission per photon, known as the quantum efficiency (QE) and also increased the extracted longitudinal polarization to approximately $88 \%$.

The QE is an important diagnostic and is measured daily. Several experimental properties are empirically related to QE. For example, due to the Gaussian intensity profile of the laser spot, over time a QE hole can form, where the likelihood of an emitted electron is higher along the spot sides. This results in changes in the electron bunch spatial distribution, possibly in a helicity dependent way. Therefore, when the QE drops below some nominal value $(\sim 0.3 \%)$ the beam spot on the cathode is moved. The development of a QE hole is illustrated in Figure 3.4.

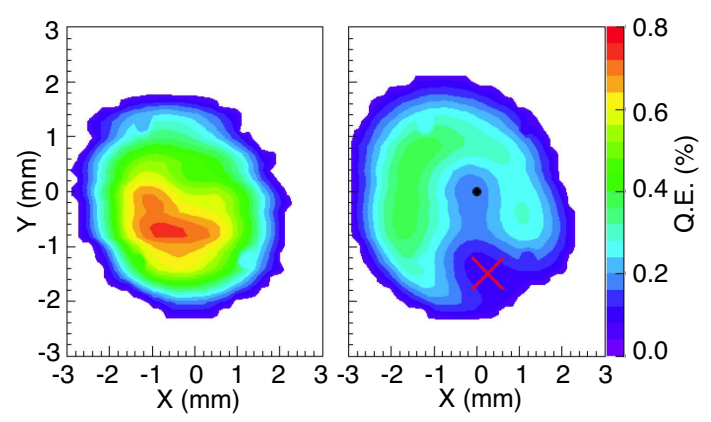

Figure 3.4: Quantum efficiency scans before (left) and (right) after a month of highcurrent running. The dot in the right-hand figure is the electro-static center, while the $\mathrm{X}$ shows the beam spot center. The cathode active area was $\sim 5 \mathrm{~mm}$ in diameter while the laser spot size was $1 \mathrm{~mm}$ in diameter. Reproduced from [67].

The dominant mechanism for QE degradation is backwards ion bombardment [69]. To the reduce this in Run 2, the laser spot size was increased from $0.5 \mathrm{~mm}$ to $1.0 \mathrm{~mm}$. During Run 1, the smaller spot size provided modest charge-lifetimes of $\sim 50 \mathrm{C}$ per laser spot, while cathode lifetimes of almost $200 \mathrm{C}$ were achieved in Run 2. Before $Q_{\text {weak }}$ Run 2, low-QE was empirically linked to beam polarization changes. Some evidence of this is seen in Run 1, but not in Run 2. This may be related to the increased spot size, but this has not been proven. 
Each hall has its own pulsed gain-switched diode laser operating at $1560 \mathrm{~nm}$, which gets frequency doubled to $780 \mathrm{~nm}$ in a nonlinear crystal. Each laser operates at 499 $\mathrm{MHz}$, and is sequentially pulsed $120^{\circ}$ out-of-phase from the others, so that each experimental hall receives beam simultaneously. The beams were combined using a polarizing cube, such that one of the high-current halls has opposite polarization to the others. In this case, Hall C had opposite polarization to Halls A and B.

The linearly polarized laser light is converted to circularly polarized light via a Pockels Cell (PC), which is a birefringent crystal where the degree of birefringence is proportional to an applied voltage. The $\mathrm{PC}$ was aligned with its birefringent axis $\sim 45^{\circ}$ to the incident light. In this configuration, at $\sim 2.5 \mathrm{kV}$, the $\mathrm{PC}$ acts as quarter-wave plate, converting the linearly polarized light into the circularly polarized light needed to produce longitudinally polarized electrons. Reversing the applied voltage reverses the birefringence, and therefore reverses the electron's polarization vector.

Despite care in setting up the PC alignment and voltage settings, residual linear polarization did leak into the signal. This caused unwanted helicity-correlated transport asymmetries, such as charge asymmetry extracted between the two helicity states. To minimize these effects, an automated charge feedback system was used to make small adjustments to the $\mathrm{PC}$ voltage.

The PC, the primary method of helicity reversal in $Q_{\text {weak }}$, was flipped at $960 \mathrm{~Hz}$. Groups of four helicity patterns, called quartets, were produced, where the first and last helicity states were identical and the middle two states were opposite to the first (that is, patterns of +--+ or -++-$)$. The experimental asymmetries were formed from these quartets, with the initial helicity state being pseudo-randomly chosen. This quartet structure was chosen to suppress linear drifts in the electronics chain.

When the high-voltage to the PC is switched, the crystal itself rings before settling, causing changes in both the polarization and the charge asymmetry; this takes approximately $60 \mu \mathrm{s}$. Therefore, after each helicity flip, the data acquisition is gated off for $70 \mu \mathrm{s}$ to let the crystal settle, creating a $6.7 \%$ dead time. The quartet patterns are reported to the data acquisition, but are temporally delayed by two quartets to isolate the detector signals from the injector, because even weak couplings can result in significant false asymmetries.

As seen in Figure 3.3, an insertable half-wave plate (IHWP) is placed immediately before the PC. This element was inserted every 4 or 8 hours to switch the sign of the experimentally produced electrons for a given PC voltage. ${ }^{1}$ Each 4 - or 8 -hour segment

\footnotetext{
${ }^{1}$ The addition of the half-wave plate essentially de-couples the applied PC voltage from the produced
} 
of data was called a slug. Assuming an equal amount of data was taken in both halfwave plate states, many helicity-correlated properties would naturally cancel when pairs of slugs are combined. This is one type of slow helicity reversal used in $Q_{\text {weak }}$. The others include solenoid flippers (Section 3.2.2) and electron $(g-2)$ procession from multi-pass running (Section 3.2.3).

\subsubsection{Injector and slow helicity reversals}

Once the electrons are produced, an electro-static field brings them into the injector. The purpose of the injector is to accelerate the produced electron bunches and condition them before entering the main accelerator. For high-current beam, such as required in $Q_{\text {weak }}$, controlling Coulomb-induced space charge effects can be difficult, and longitudinal bunching is required. While in the injector, the electrons are accelerated to 62 $\mathrm{MeV}$ using the superconducting cavities discussed in Section 3.2.3.

To limit the emittance, both transverse and temporal (longitudinal) dimensions were trimmed using a series of apertures and a pair of $499 \mathrm{MHz} \mathrm{RF}$ deflecting cavities known as a chopper. The chopper was phased to sweep the beam in a circle with revolution frequency of $499 \mathrm{MHz}$. Throughout $Q_{\text {weak }}$ the chopper aperture was kept fully open $\left(\approx 20^{\circ}\right)$, since losses could be significant due to space charge effects.

The injector included a $4 \pi$ spin manipulator consisting of two Wien filters separated by two solenoid magnets. The first (vertical) Wien filter is oriented to rotate the spin polarization vertically (out of the accelerator plane). The two inner solenoids are set to rotate the spin vector either left or right, while maintaining constant beam focusing and envelope. This then rotates the spin vector into the horizontal plane, with the electron's spin vector orthogonal to its direction of motion. Finally the second (horizontal) Wien filter is oriented to rotate the spin vector $\pi / 2$ in the horizontal plane, producing longitudinally polarized electrons. By switching the sign of both solenoid magnets, the electron helicity can be flipped [72]. This two Wien filter system also enables canceling of spin precession induced by the other transport magnets.

Although the solenoid fields are rotated, this procedure is colloquially known as a Wien flip, and is the second example of a slow helicity reversal (first discussed in Section 3.2.1). Wien flips de-correlate the laser helicity from that of the experimentally produced electrons, canceling another class of potential helicity-correlated effects. The most well known is a beam breathing mode where the electron beam expands or contracts in a systematic, helicity correlated manner.

electron's helicity. 


\subsubsection{Accelerator}

After the injector, the polarized electrons enter the main accelerator facility. This consists of 2 re-circulating linacs and 2 bend arcs. The electron beam can be recirculated a total of 5 times, or passes, for a total energy of approximately $6 \mathrm{GeV}$. The main component of beam acceleration in the linacs is the SRF cavity. Each cavity is $0.1 \mathrm{~m}$ long, consists of $5 \mathrm{SRF}$ cells, and has an average accelerating gradient of $7.5 \mathrm{MV} / \mathrm{m}$. The cavities are manufactured in-house from pure niobium sheet which is drawn into cups, chemically cleaned, and welded together [70]. Groups of 8 cavities form a cryomodule. Each linac employs 20 cryomodules and produces an acceleration of $548 \mathrm{MeV}^{2}$. This doesn't include the $62 \mathrm{MeV}$ provided by the injector.

After leaving the injector, the electrons travel near the speed of light $(\beta \sim 0.99997)$. Since at such relativistic speeds their velocity is nearly independent of energy, they can stay in phase with higher-energy electrons having previously undergone multiple machine passes. At the end of both linacs, the beam enters chromatic dipole spreader magnet which physically separates the beam into its different energy components (e.g., 1 -pass beam is separated from higher-pass beams). The beam is bent $180^{\circ}$ and then re-combined before entering the other linac. In the South Linac, RF separators are used to deflect the beam into the appropriate halls as needed. Downstream of the beam switch yard another array of magnets is used to steer the beam and correct for any aberrations. This setup allows all 3 experimental halls to receive 5-pass beam concurrently; alternatively, different halls can receive longitudinally polarized beam of different energies depending on certain kinematic restrictions [73].

The $Q_{\text {weak }}$ experiment ran almost exclusively with single pass beam of $1.16 \mathrm{GeV}$. Short periods of 3-pass beam at $3.3 \mathrm{GeV}$ and 1-pass beam at $880 \mathrm{MeV}$ were used for systematic studies, and a short period of 2-pass beam at $1.16 \mathrm{GeV}$ provided another useful check of independent helicity reversal from $(g-2)$ procession relative to 1-pass operation [67].

\subsection{Beam diagnostics}

During transit through the accelerator and into the experimental halls, a diverse array of tools are implemented to monitor important beam properties. For JLab's parity program these tools take on additional significance, as small changes in beam properties

\footnotetext{
${ }^{2}$ NB: Since the completion of $Q_{\text {weak }}$, Jefferson Lab has upgraded its energy capability from a maximum of $6 \mathrm{GeV}$ electrons to $12 \mathrm{GeV}$. Therefore, many current publications quote greater cavity properties.
} 


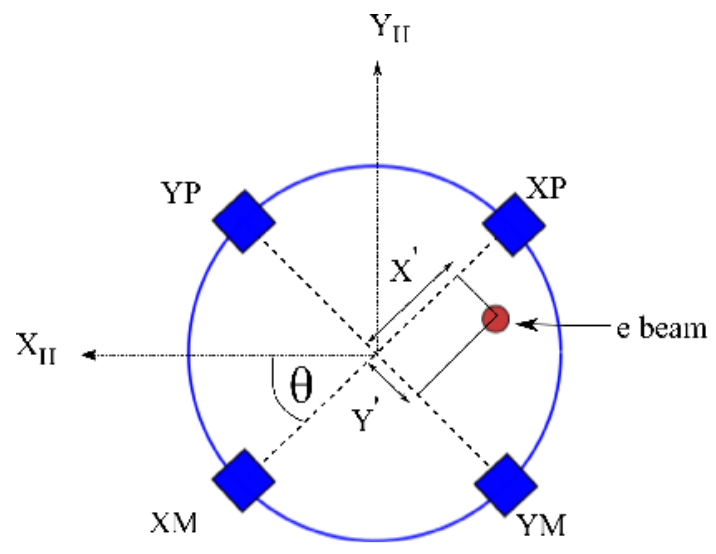

Figure 3.5: Beam position monitor (BPM) coordinate system (dotted lines). The BPM coordinate system is rotated $45^{\circ}$ with respect to the nominal Hall coordinate system. This was a design choice to help minimize the possible effects of synchrotron radiation in the arc. Reproduced from [75].

can have profound effects on the experimentally measured asymmetry.

The most important diagnostics monitor the beam's position, current and charge asymmetry, energy, polarization, and any helicity-correlated changes in these properties. In this section we describe the improvements to the Hall $\mathrm{C}$ beam position monitors (BPMs), various beam current monitors (BCMs), and the ways of monitoring beam energy. A section on correcting helicity-correlated beam properties with linear regression and a beam modulation system is presented. Monitoring of beam polarization is covered in Section 3.4.

\subsubsection{Beam position monitors}

The beam position monitors (BPMs) consist of two sets of opposing quarter-wave pickup antennas (one set each for $X$ and $Y$ directions). The antennas are transverse to the beam direction and centered on the beam axis; they are rotated at $45^{\circ}$ to reduce possible synchrotron radiation effects in the arc [74]. Figure 3.5 shows a schematic of the typical Jefferson Lab BPMs and their coordinate system. The antennae pick up either the 499 $\mathrm{MHz}$ or $1497 \mathrm{MHz}$ of the bunch frequency depending on location [75].

The general operating principle is that the antennae voltage signal is proportional to distance from the beam. For example, as the beam drifts closer to $X^{+}$its voltage output is increased while the signal from $X^{-}$decreases. Assuming perfectly matched gain parameters between the $X$ and $Y$ wires, the central position of the beam is given by:

$$
X^{\prime}=\kappa \frac{X^{+}-X^{-}}{X^{+}+X^{-}}
$$

where $\kappa$ is the sensitivity of the BPM at the bunch frequency [74]. $\kappa$ also contains a 
conversion factor to convert the signal to $\mathrm{mm}$ [75].

The beam position and angle at the $Q_{\text {weak }}$ target are determined using a series of beam position monitors in the drift region immediately preceding the target. BPM $3 \mathrm{c} 12$ is located in the area of the largest dispersion in the Hall $\mathrm{C}$ arc, where it can conveniently double as a relative beam energy monitor.

Periodically, low-current runs were taken using the superharp, which is another monitor of absolute beam position. The superharp is a series of three wires which move transversely across the beam, enabling precision measurements of the beam's profile $(\Delta x \sim 10 \mu \mathrm{m})[76]$. Due to the invasive nature of this process, Superharp scans are limited to low-current systematic studies, or as a diagnostic tool to determine if the beam has non-Gaussian tails.

\subsubsection{Beam current monitors}

Experimental Hall C employs two types of beam current monitors: pillbox RF cavities used to continuously monitor relative changes in beam current, and a parametric current transformer (the Unser) monitor for absolute beam current determination. Seven of the pillbox cavity type current monitors were installed for $Q_{\text {weak }}$ [77]. Five were used in Run 1. BCM1 and 2 used analog receivers and were the main charge monitors for Run 1 , and also served as the primary monitor during low-current event-mode studies during both Run periods. BCM17 was main beam current monitor during the Møller beam polarization measurements. In Run 2, an additional two BCMs were installed which used digital receivers. In Run 2, BCM8 was the primary monitor used to normalize the main detector signal with respect to charge [78].

The relative current monitors were stainless steel cylindrical cavities resonant to the $\mathrm{TM}_{010}$ mode at $1497 \mathrm{MHz}$, identical to the electron beam delivery frequency. The BCMs were designed to operate at the third harmonic of the bunch frequency in the Hall, as this is relatively insensitive to beam position [79]. Therefore, as the electron beam passed through, the cavity resonates. Since the resonant frequency is highly sensitive to the cavity's physical dimensions, they were thermally stabilized to $43^{\circ} \mathrm{C}$ to reduce temperature dependence.

The cavity power is extracted using a wire loop antenna, where the power output is proportional to the beam current squared (i.e., $P \propto I^{2}$ ). This high frequency signal was ultimately down-converted to $100 \mathrm{kHz}$, and then fed into an ADC module. The signal is also sent to a $1 \mathrm{MHz}$ voltage-to-frequency converter and a set of scalers for low-current tracking measurements [67, 42]. 
The resonant cavity model provides stable, low noise, continuous relative measurements of beam currents (relative because the signal is proportional to beam intensity). These were calibrated against a parametric current transformer [80], which was located between BCMs 1 and 2. Essentially, the Unser monitor is a toroidal donut that sits around the beam pipe. The electron beam induces a net magnetic field in the toroid, which is measured by a transformer. The total flux is then driven to zero using a second transformer; the current required by this transformer is proportional to the beam current. The gain of the Unser monitor is known to be linear and stable, but the offset (pedestal) has large drifts. Therefore, calibration must be carefully performed using a series of beam-off periods to determine the offset.

The BCM resolution is much smaller than the intrinsic beam jitter, so a better gauge of BCM performance uses multiple BCMs. The preferred metric, called the double difference, eliminates common-mode noise between the two monitors, and removes fluctuations in beam current, resulting in a metric sensitive only to the resolution of the BCM pair. For example, the double difference $(D D)$ between BCM1 and 2 is given by

$$
D D_{12}=\frac{Q_{1}^{+}-Q_{1}^{-}}{Q_{1}^{+}+Q_{1}^{-}}-\frac{Q_{2}^{+}-Q_{2}^{-}}{Q_{2}^{+}+Q_{2}^{-}}
$$

where $Q_{I}^{j}$ denotes the charge measured by $\mathrm{BCM} i$ for beam helicity state $j$. If the two monitor resolutions are approximately equal, the resolution for one monitor is $\sigma \approx \sigma_{D D} / \sqrt{2}$

\subsubsection{Beam energy determination}

To accurately determine the beam energy, the Hall $\mathrm{C}$ beam line is used as a spectrometer. The Hall $\mathrm{C}$ beam line, immediately after the beam switch yard, is a full 41.6 meters long and contains a series of magneto-optical elements: dipoles, quadrupoles, sextupoles, and beam correctors. The beam momentum $p$ is determined by [76]

$$
p=\frac{e}{\Delta \theta} \int B d l
$$

where $\theta$ is the bending angle through the arc and $\int B d l$ is the calibrated dipole field integral. The total deflection from the dipoles is $34.3^{\circ}$. Since the dispersion is well known in this portion, a precise measurement of beam position before and after the arc enables a precision measurement of the beam energy [81].

Beam energy determination begins with an absolute energy measurement, where all the magnets and correctors are turned off, except for the dipoles. Under these conditions the dispersion is well known to be $12 \mathrm{~cm} / \%$. The position and angle of 
the incoming beam is precisely measured using the high resolution harp wire scanners (described in Section 3.3.1). The beam direction and angle of the outgoing beam are also measured, enabling determination of the absolute beam energy to a precision of $10^{-3}[81]$.

After determining the absolute energy scale with the dipoles, the other magnetooptical elements are energized to provide the required beam to the hall. With the other magnets on, the system forms the equivalent of a second-order achromatic lens. Variations in horizontal beam position at the arc mid-point (BPM 3c12) can be used to determine relative changes in energy. The dispersion at BPM 3c12X is well known to be $4 \mathrm{~cm} / \%$. Relative energy determination on the order of $10^{-4}$ is feasible.

\subsubsection{Beam modulation}

Parity-violating experiments are particularly sensitive to subtle shifts in beam parameters, especially if these shifts correlate with the fast helicity reversal. Any small correlated shift would provide false asymmetries, which may then be enhanced by various broken symmetries or nonlinearities in the experimental apparatus. The total false asymmetry, $A_{\text {false }}$, is given by

$$
A_{\text {false }}=\sum_{i}^{N} \frac{\partial A}{\partial P_{i}} \Delta P_{i}
$$

where $\left(\partial A / \partial P_{i}\right)$ is the system sensitivity to a given beam parameter $P_{i}$. The sum runs over all the relevant beam parameters: transverse position $(X, Y)$, angle $\left(X^{\prime}, Y^{\prime}\right)$, energy $(E)$, and charge asymmetry $\left(A_{Q}\right)$. The charge sensitivity is non-zero due to percent-level nonlinearities in the detector chain; therefore the charge feedback system described in Section 3.2.1 is of considerable importance.

Correction of such helicity-correlated effects occurs from two methods. The first method uses the natural jitter and beam motion and their correlations with the measured asymmetry and beam parameters. This method uses standard multivariate linear regression techniques to determine detector sensitivities and correct all correlations away. In principle, this guarantees absence of residual correlations; however, it is unsatisfying as it provides no physical explanation or insight into the correlations. It is simply a mathematical prescription.

Although 13 regressions schemes were developed, only 9 were ultimately used. Each uses a different set of monitors or detectors to measure the beam sensitivities. Ideally, if each set spans a complete basis, differences in the corrected asymmetries would be mostly due to monitor resolution and noise. One major difference between some 


\begin{tabular}{|l|c|c|c|c|c|c|}
\hline Scheme name & $\mathrm{X}$ & $\mathrm{Y}$ & Xslope & Yslope & Energy & Charge \\
\hline std & targetX & targetY & targetXSlope & targetYSlope & energy & no \\
\hline std_5+1 & targetX & targetY & targetXSlope & targetYSlope & bpm3c12x & asym_charge \\
\hline set3 & targetX & targetY & targetXSlope & targetYSlope & energy & bpm3c12X \\
\hline set4 & targetX & targetY & targetXSlope & targetYSlope & energy & bcm5 \\
\hline set7 & bpm_9_p_4X & bpm_9_p_4Y & bpm_9_m_4X & bpm_9_m_4Y & bpm3c12X & no \\
\hline set8 & bpm_9_p_4X & bpm_9_p_4Y & bpm_9_m_4X & bpm_9_m_4Y & bpm3c12X & asym_charge \\
\hline set10 & targetX & targetY & targetXSlope & targetYSlope & energy & bcm6 \\
\hline set11 & targetX & targetY & targetXSlope & targetYSlope & bpm3c12X & no \\
\hline set13 & targetX & targetY & targetXSlope & targetYSlope & bpm3c11X & no \\
\hline
\end{tabular}

Table 3.2: Summary of regression scheme monitors. std stands for "standard."

schemes is including charge asymmetry as a regression variable. Table 3.2 summarizes the different regression schemes and what monitors they use to extract beam parameter sensitives.

The notation used in Table 3.2 was shorthand for a combination of weighted monitors developed during analysis. The position monitors denoted by "target" are a weighted combination of five BPMs just upstream of the target. The notation in sets 7 and 8 are

$$
\begin{aligned}
\text { bpm_9_p_4Xp } \equiv \text { bpm3H09bX }+ \text { bpm3h04X } & \text { (position }- \text { like }) \\
\text { bpm_9_p_4Xm } \equiv \text { bpm3H09bX - bpm3h04X } & \text { (slope }- \text { like }),
\end{aligned}
$$

with similar definitions for the Y monitors. Energy is typically monitored by BPM3c12X, which is located in the arc where the dispersion is largest. However, during a short period of running on an aluminum target the electronics read-out for this BPM failed. Therefore, regression scheme set13 was developed using BPM3c11X, which is also located in the arc, but with approximately half the sensitivity to beam energy.

The second method of correcting helicity-correlated beam changes is to actively modulate, or kick, the beam in a controlled way using an external driving signal and determine the detector sensitivities from their response. In effect, by driving each parameter separately, modulating the beam should in theory provide cleaner extraction of the detector sensitivities.

The beam modulation system used during $Q_{\text {weak }}$ includes two pairs of magnetic coils placed in the Hall $\mathrm{C}$ beamline upstream of the arc. These are used for beam position and angle modulation. Beam energy modulation is accomplished by driving a small voltage signal to an SRF cavity; by changing the electric field the beam energy also changes. First, each beam parameter is independently modulated for 4 seconds with a frequency of $125 \mathrm{~Hz}$. Between each modulation micro cycle, there is a period of 
approximately 60 seconds reconfiguration time during which production data is taken. The total time to measure one complete set of all five beam parameters $\left(X, Y, X^{\prime}, Y^{\prime}\right.$, and $E$ ) is 320 seconds. There is no a priori reason to discard the modulated data after extracting the sensitivities; in principle they should provide equally fine asymmetry measurements as regular production running.

Two recent $Q_{\text {weak }}$ theses focused on the beam modulation and linear regression subsystems. The interested reader is directed to references $[82,83]$ for further details.

\subsection{Polarimetry}

Precise determination of electron beam polarization is important for today's experiments. This is particularly true for Jefferson Laboratory's parity program, including $Q_{\text {weak }}$, where electron beam polarizations near $100 \%$ are desirable. $Q_{\text {weak }}$ required knowledge of the beam polarization to $1 \%$ relative precision. To meet this goal, $Q_{\text {weak }}$ used two complementary devices: a Møller polarimeter and a Compton polarimeter.

An in-depth treatment of the Møller polarimeter analysis is the focus of Chapter 4. A description of both Run 1 and Run 2 analysis methods will be found there, as well as a full treatment of the systematic uncertainties. Both polarimeters are described below.

\subsubsection{Møller Polarimeter}

Electron-electron, or Møller, scattering is an ideal probe to determine the absolute electron beam polarization as it is a pure quantum electrodynamic process (QED). The cross section is large, well-known, and calculable to high precision [84, 85]. For scattering of longitudinally polarized electrons, the spin-dependent Møller cross section in the center of mass frame is given by:

$$
\frac{d \sigma}{d \Omega}=\frac{d \sigma_{0}}{d \Omega}\left[1+P_{Z}^{T} P_{Z}^{B} A_{Z Z}(\theta)\right]
$$

where $\frac{d \sigma_{0}}{d \Omega}$ is the unpolarized cross section, $A_{Z Z}(\theta)$ is the analyzing power, and $P_{Z}^{T}$ and $P_{Z}^{B}$ are the longitudinal polarizations of the target and beam, respectively. At highenergy, the unpolarized cross-section in terms of the center-of-mass scattering angle, $\theta$, is [84]:

$$
\frac{d \sigma_{0}}{d \Omega}=\left(\frac{\alpha\left(4-\sin ^{2} \theta\right)}{2 m_{e} \gamma \sin ^{2} \theta}\right)^{2}
$$


where $m_{e}$ is the electron rest-mass, $\alpha$ is the fine-structure constant, and $\gamma$ is the relativistic Lorentz factor. The analyzing power is

$$
A_{Z Z}(\theta)=-\sin ^{2} \theta \frac{\left(8-\sin ^{2} \theta\right)}{\left(4-\sin ^{2} \theta\right)^{2}} .
$$

Figure 3.6 shows the analyzing power magnitude as a function of center-of-mass scattering angle. The analyzing power is maximal at $90^{\circ}$ center-of-mass; the slow variation makes it an ideal region to measure in. The blue dotted lines give the approximate \pm $3^{\circ}$ acceptance in the Hall C Møller.

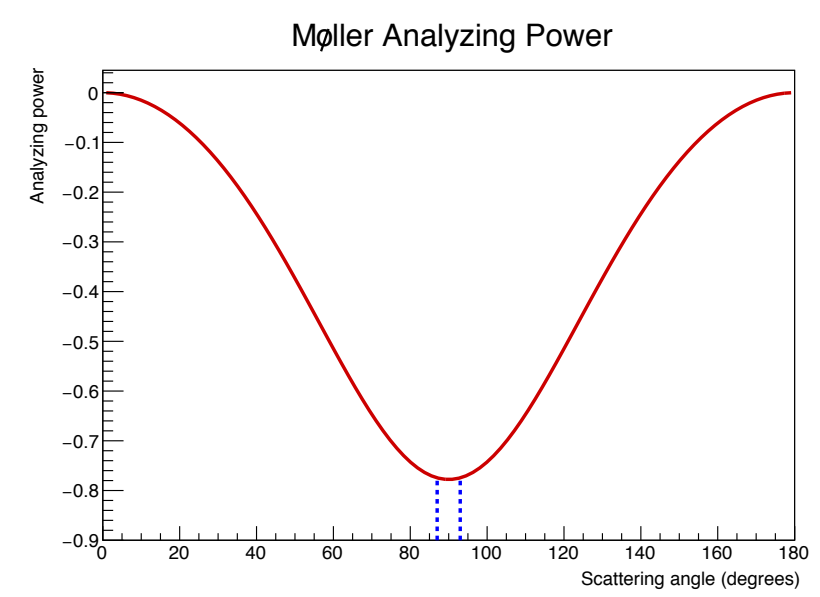

Figure 3.6: Møller analyzing power versus center-of-mass scattering angle.

To determine the polarization, one directly exploits the helicity dependence in equations (3.7) by forming an experimental asymmetry. This selects only the helicity dependent piece:

$$
A_{\text {exp }}=\frac{\left(\frac{d \sigma}{d \Omega}\right)^{\uparrow \uparrow}-\left(\frac{d \sigma}{d \Omega}\right)^{\uparrow \downarrow}}{\left(\frac{d \sigma}{d \Omega}\right)^{\uparrow \uparrow}+\left(\frac{d \sigma}{d \Omega}\right)^{\uparrow \downarrow}}=\left|P_{Z}^{T}\right|\left|P_{Z}^{B}\right| A_{Z Z}(\theta) .
$$

One can easily measure $A_{\text {exp }}$ by determining the cross-section asymmetry for beam and target spins aligned parallel $(\uparrow \uparrow)$ and anti-parallel $(\uparrow \downarrow)$. At $90^{\circ}$ center-of-mass both the analyzing power and cross section are large $\left(A_{Z Z}(90)=-\frac{7}{9}\right.$ and $\left.\frac{d \sigma_{0}}{d \Omega}=17.9 \mathrm{fm}^{2} \mathrm{sr}^{-1}\right)$, so the beam polarization can easily be determined if the target polarization is well known. Therefore, the key is selecting an appropriate polarized electron target.

Unlike previous Møller polarimeter designs, the Hall C Møller uses a pure iron foil target, which is forced into magnetic saturation out-of-plane using a high-field superconducting solenoid. The main components of the polarimeter are:

- A thin, pure iron-foil target.

- A superconducting solenoid magnet, used to magnetically saturate the target 
foil out-of-plane. For $Q_{\text {weak }}$, the solenoid ran at 3.5 Tesla, well above the $2.2 \mathrm{~T}$ magnetic saturation point.

- Two quadrupole magnets acting as an optical lens. These focus the Møller electrons at $90^{\circ}$ center-of-mass, where the asymmetry is largest, onto the detector array.

- Two lead-glass calorimeters detect both the scattered and recoil electrons in coincidence. A narrow timing gate of 5 ns reduces backgrounds from single arm Mott scattering. The acceptance is defined by collimators positioned immediately before the calorimeters.

- A set of scintillator hodoscopes immediately in-front of the acceptance defining collimators. These are used to determine the position of the electrons entering the detectors, and also to verify the polarimeter quadrupole settings by looking at the position correlation between both detectors.

- A set of movable collimators between the two quadrupoles. These are used to further reduce backgrounds and do not affect the acceptance.

Because of possible heating and depolarization of the target foil, Møller measurements are invasive and limited to low-currents below $\sim 2 \mu \mathrm{A}$.

Figure 3.7 shows the original Run 1 layout. Before $Q_{\text {weak }}$, an additional quadrupole (Q2) was added to the beamline in preparation for the upcoming Jefferson Lab $12 \mathrm{GeV}$ upgrade. It was un-powered during Run 0 and Run 1.

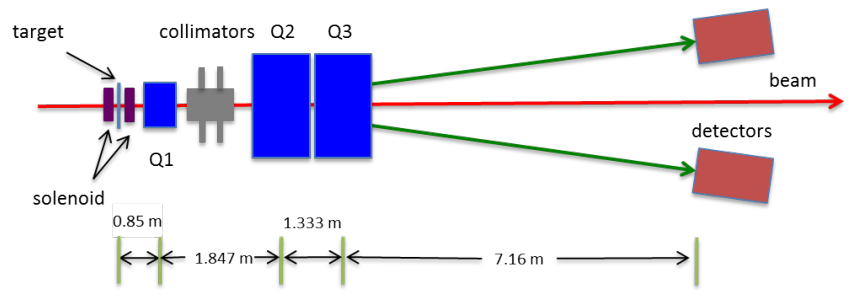

Figure 3.7: Layout of the Møller polarimeter for Run 1. The second quadrupole, Q2 was installed for the $12 \mathrm{GeV}$ upgrade and was not used during $Q_{\text {weak }}$. Reproduced from $[86]$.

During Run 1 it became clear that our optics system was not functioning correctly. ${ }^{3}$ Near the end of Run 1, Hall probes were placed inside the original Q3 to determine how the field varied. Great effort was undertaken to understand the effect of this on

\footnotetext{
${ }^{3}$ It is interesting to note the first concrete evidence of this came from the accelerator operators. They noticed that over time the beam downstream of the Møller polarimeter was moving on its own, requiring periodic beam steering.
} 
the Run 1 analyzing power, and is the subject of Section 4.2.6. Measurements between Run 1 and 2 verified the problem with the coils in Q3, and also determined that Q2 was functional. Q3 was removed for Run 2, and Q2 was shifted downstream to replace it. Q2 was then renamed Q3 to avoid confusion.

\subsubsection{Compton polarimeter}

To supplement the Møller polarimeter, the $Q_{\text {weak }}$ collaboration installed a Compton polarimeter. Like Møller scattering, Compton $(e \gamma)$ scattering is pure QED, and thus precisely calculable. Unlike the Møller polarimeter, however, the Compton polarimeter is neither invasive nor current-limited, and thus offers a precise, continuous monitor of beam polarization at the high currents required by $Q_{\text {weak }}$. The Hall C polarimeter includes both photon and electron detectors, enabling two quasi-independent determinations of beam polarization. The statistical precision of the Compton electron detector was $\leq 1 \%$ /hour with beam currents of $180 \mu \mathrm{A}$.

Similar to Møller polarimetry, the beam polarization is determined by measuring the asymmetry between the helicity-dependent cross-sections

$$
A_{m s r}=P_{e} P_{\gamma} A_{Z Z}
$$

where $A_{m s r}$ is the measured asymmetry, $P_{e}$ is the beam polarization, $P_{\gamma}$ is the photon polarization produced by a circularly polarized laser, and $A_{Z Z}$ is the system's analyzing power. Therefore if $P_{\gamma}$ is well determined and $A_{Z Z}$ calculable, $P_{e}$ is directly calculable.

Figure 3.8 is a schematic of the Hall $\mathrm{C}$ Compton polarimeter. The polarimeter consists of 4 dipole magnets wired in series, a laser system, a photon detector, and an electron detector. The first dipole pair steers the beam downward by $\sim 57 \mathrm{~cm}$ and parallel to the original beam line. Here the beam encounters the laser system: a 10 Watt, $532 \mathrm{~nm}$ (green) continuous-wave laser coupled to a low-gain Fabry-Perot cavity yielding greater than $1 \mathrm{~kW}$ of stored power in the interaction region. The light is circularly polarized, but a half-wave plate and quarter wave plate were used to flip the polarization direction for systematic checks; this is similar to how the main experiment implements slow helicity reversals (see Sections 3.2.2 and 3.2.3). The full laser system is described in [83]. After passing through the interaction region, the second set of dipoles bends the electron beam upward, in line with the original beam line. The use of dipole bending magnets facilitates back-scattered photon detection, as well as providing a convenient way of accessing the Compton-scattered electron, which due to the momentum exchange has a different bend radius in the third dipole. 


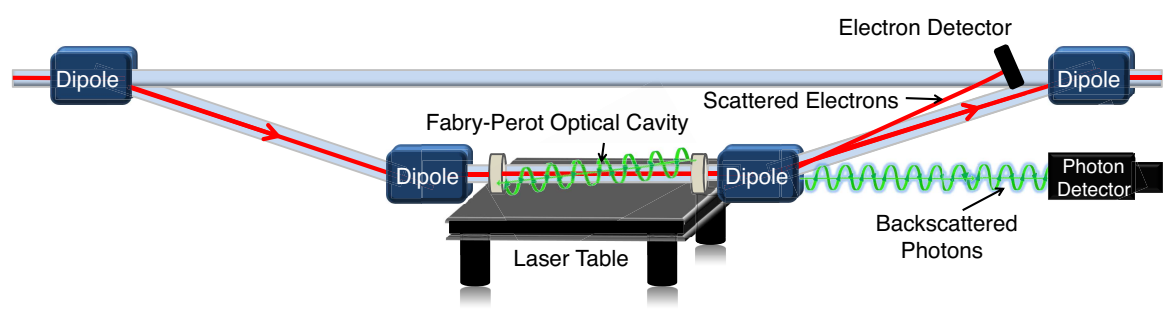

Figure 3.8: Layout of the Hall C Compton polarimeter [83].

After exiting the third magnet, the scattered electron enters the detector, consisting of a series of 4 diamond micro-strip detectors. Each detector plane has 96 strips, each $200 \mu \mathrm{m}$ wide. The electron detector became operational late in Run 1 (April 2011), and increasingly became a key instrument during Run 2. The Møller was still used during Run 2, making periodic polarization measurements. The Compton electron detector is described in detail in [87].

The photon detector was initially designed for use with a CsI crystal, which would detect backscattered photons with energies ranging from 10-50 MeV. Sadly, the original crystal suffered from large phosphorescence (after glow), leading to significant effects on the measured asymmetry, even with beam off. The crystal was ultimately replaced with lead-tungstate $\left(\mathrm{PbWO}_{4}\right)$, which was cooled at $14^{\circ} \mathrm{C}$ to increase the signal-to-noise ratio. The photon detector analysis is discussed in full in [88].

\subsection{Targets}

The $Q_{\text {weak }}$ experiment's primary target was liquid hydrogen $\left(\mathrm{LH}_{2}\right)$, however an array of other targets were used for various systematic studies. In particular, several solid targets (such as aluminum) were used to determine the effect of the primary target cell in the main asymmetry measurement. These solid targets were part of a target matrix mounted directly below the main target cell. The full details and calculations of the target wall contribution is the subject of Chapters 5 and 6 .

\subsubsection{Primary LH2 target}

The primary $\mathrm{LH}_{2}$ target consisted of a hydrogen re-circulating pump, a heat exchanger responsible for liquefying the hydrogen and removing any energy deposition from the electron beam, and a heater to regulate the $\mathrm{LH}_{2}$ temperature and replace the beam power when the beam was off. The target cell also contained thin windows, which were inside the experimental acceptance and contributed to the overall signal. The target cell length was $34.4 \mathrm{~cm}$ and held approximately 55 liters of $\mathrm{LH}_{2}$. The target operated 


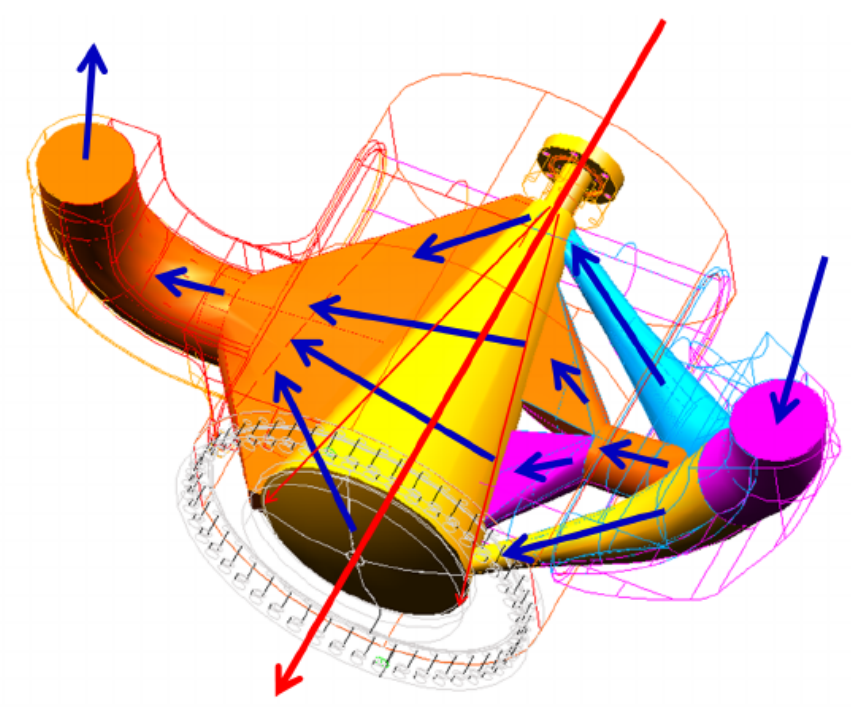

Figure 3.9: The primary $Q_{\text {weak }}$ target Computational Fluid Dynamics (CFD) design diagram. The red arrow is the electron beam while the blue arrows are the flow of $\mathrm{LH}_{2}$. Reproduced from [67].

at $20 \mathrm{~K}$ and 35 psia ( 2.4 atmospheres), and the hydrogen itself was $99.999 \%$ pure [78].

The target cell entrance and exit windows were constructed of the high-strength aluminum alloy $\mathrm{Al}$ 7075-T6. This alloy is predominately pure aluminum (87-91\%), but also contains zinc (5.1-6.1\%), magnesium (2.1-2.9\%), copper (1.2-2.0\%), and trace amounts of other contaminants $(\leq 0.5 \%)$ [89]. As these windows were an undesirable source of background, they were designed to be as thin as possible while still maintaining safe operation. The entrance window was $96.5 \mu \mathrm{m}$ thick, while the downstream window was $127 \mu \mathrm{m}$. The total amount of aluminum, $223.5 \mu \mathrm{m}$, corresponds to $0.25 \%$ of a radiation length. Target cell design drawings are shown in Figures 3.9 and 3.10.

The $1.16 \mathrm{GeV}$ electron beam deposited $2.1 \mathrm{~kW}$ of power along the target length due to ionization energy loss. Additional losses from viscous heating, pump heat, conductive and radiative heat load, and some reserve power increased the total heat supplied to approximately $2.5 \mathrm{~kW}$ of power. This culminated in the $Q_{\text {weak }}$ target being the highest power cryotarget ever built [90].

With nearly $200 \mu \mathrm{A}$ of beam incident on the target supplying over $2 \mathrm{~kW}$ of power, target density fluctuations (also known as boiling) could cause large fluctuations in the main detector asymmetry width. Monitoring this width ultimately provided the primary metric for the target's overall performance. Although multiple techniques were developed to characterize this metric, the most robust and clean technique was by varying the target pump speed and observing the change in main detector asymmetry 


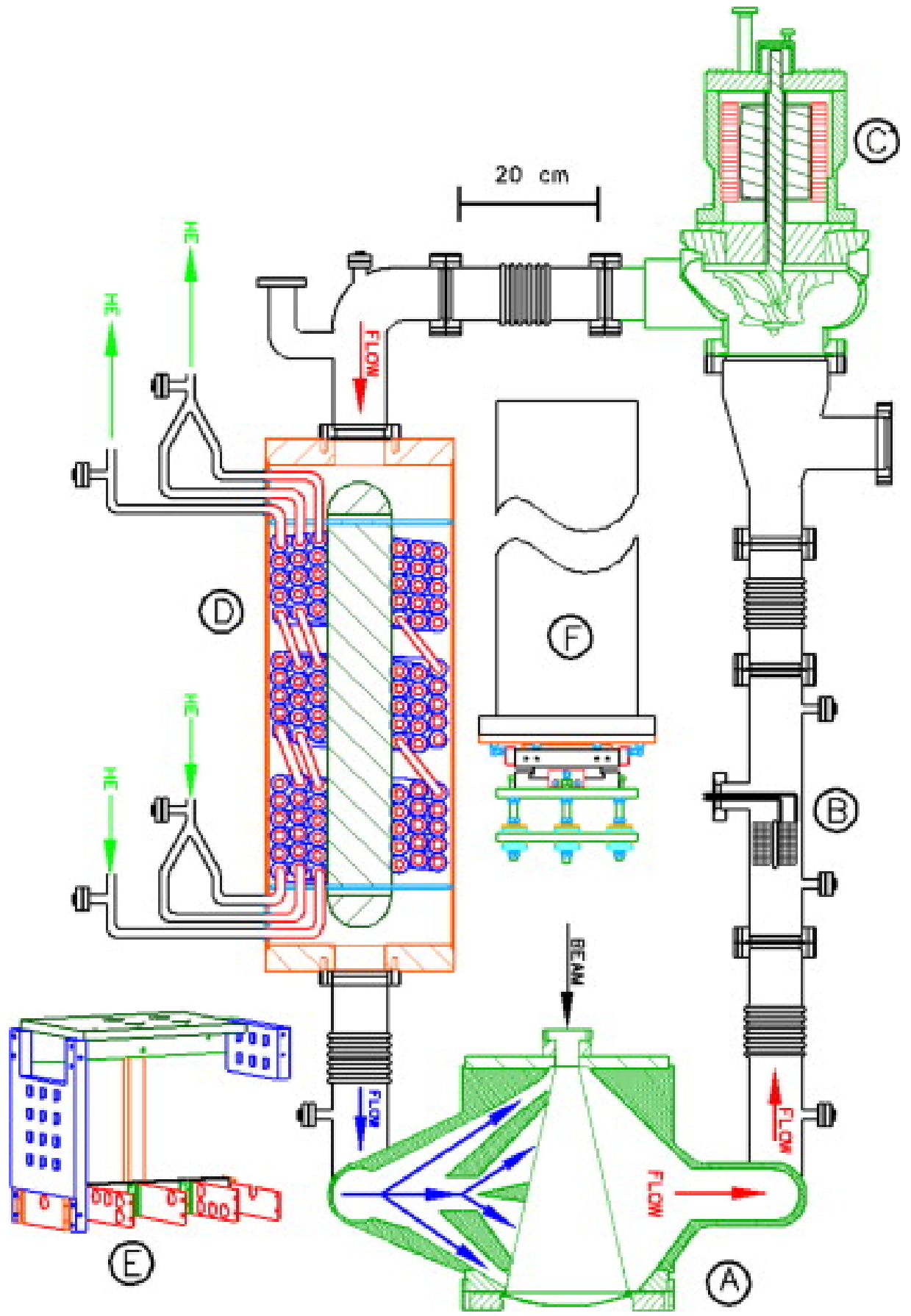

Figure 3.10: Design diagram of the full primary $Q_{\text {weak }}$ target apparatus: (A) the beam interaction region; (B) the heater; (C) the centrifugal re-circulation pump; (D) the heat exchanger; (E) the solid target ladder was mounted directly below the cell; (F) mechanical support pipe. Reproduced from [67]. 


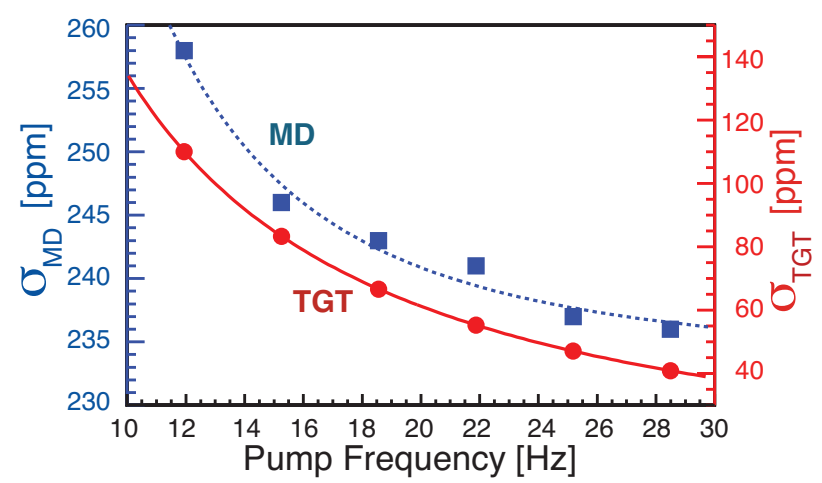

Figure 3.11: The main detector asymmetry width measured at varying rotational speeds of the $\mathrm{LH}_{2}$ re-circulation pump (blue square points, dotted line, left axis). The empirically determined fit is $\sigma_{M D}^{2}=\sigma_{A}^{2}=233^{2}+1889^{2} / f^{2.288}$ where $f$ is the pump speed. Operating conditions here were $169 \mu A$ with a $4 \times 4$ raster. The statistical contribution to the main detector width from the target is shown in red (solid circles, solid line, right axis). Reproduced from [67].

width (Figure 3.11). At the nominal conditions of the experiment, the target noise contribution was limited to $53 \mathrm{ppm}$; this is negligible when added in quadrature to the raw statistical width of 230 ppm, per quartet.

\subsubsection{Aluminum targets}

Aside from the primary $\mathrm{LH}_{2}$ target, $Q_{\text {weak }}$ used myriad solid targets, including thick "dummy" targets, optics targets, and centering targets. These were used for systematic studies throughout experimental running. Photographs of the ancillary target ladder are shown in Figure 3.12. The target ladder has both an upstream and downstream frame, with each frame having three target columns. The upstream face of the upstream target ladder aligns the targets with the upstream target window, while the upstream face of the downstream target window aligns them with the target exit window. Vertical and horizontal motion mechanisms enable access to each target.

Of particular importance were a series of thick aluminum dummy targets, which were used to measure the parity-violating asymmetry of electron scattering from aluminum. These results were used both to characterize the contribution of the aluminum target windows and, ultimately, extract the parity-violating asymmetry of aluminum. This section will focus on properties of these aluminum targets, while their measured asymmetry is the focus of Chapter 6.

The aluminum dummy targets were machined from the same high-strength aluminum alloy as the target windows. Three thicknesses of aluminum targets were in- 

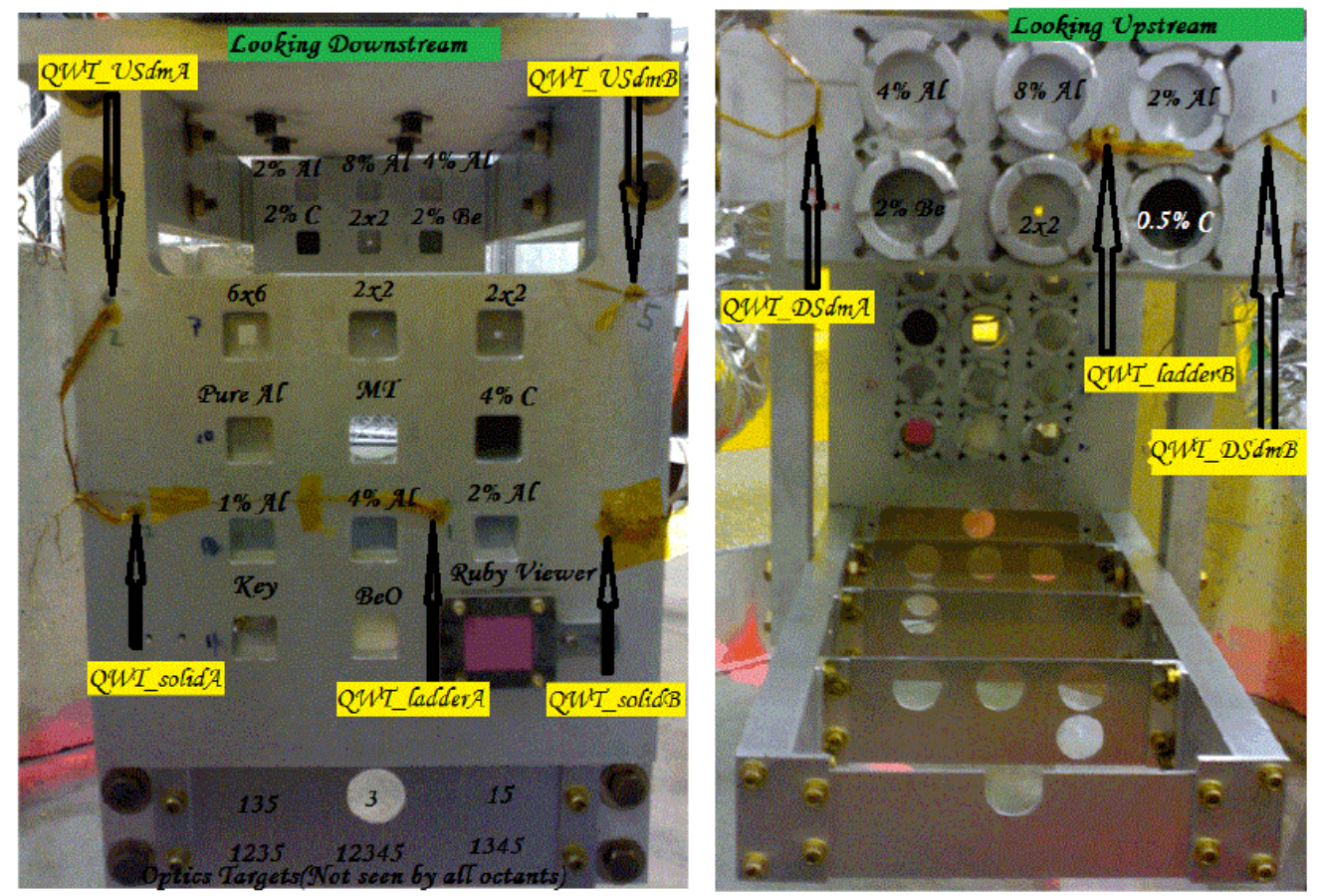

Figure 3.12: Photograph of the $Q_{\text {weak }}$ target ladder. (left) The upstream target frame. (right) The downstream target frame (looking upstream).

stalled in both the upstream and downstream ladder frames. Measurements on a variety of thicknesses enabled precise measurements of both radiative and acceptance effects. In the upstream frame there are $1 \%, 2 \%$, and $4 \%$ nominal radiation thick targets as well as a pure aluminum target. Although little data was taken on the pure aluminum target, it was added to study the contribution of the other high- $Z$ components in the aluminum alloy. The downstream frame holds $2 \%, 4 \%$, and $8 \%$ nominal radiation length targets.

Measurements of the targets were made by the $Q_{\text {weak }}$ target group to provide thickness, area, mass, and areal density of all targets. The results of these target measurements are shown in Table 3.3. For reference, the radiation length of pure aluminum is $X_{0}=24.028 \mathrm{~g} / \mathrm{cm}^{2}$.

\subsection{Collimators}

The experiment was carefully designed to handle the extraordinarily high radiation levels developing from high currents on a long, dense target. Aside from choosing radiation hard materials for the detectors and target windows, a series of collimators and shielding was employed around the target and beamline components to reduce background radiation from secondary scattering. 


\begin{tabular}{|c|c|c|c|c|c|c|}
\hline Run & Location & Target & $\begin{array}{l}\text { Thickness } \\
\text { (mm) }\end{array}$ & $\begin{array}{l}\text { Mass } \\
(\mathrm{g})\end{array}$ & $\begin{array}{l}\text { Area } \\
\text { in }^{2}\end{array}$ & $\begin{array}{l}X_{0} \\
(\%)\end{array}$ \\
\hline 1 & DS & Al $2 \%$ & $1.7725 \pm 0.00435$ & $3.0957 \pm 0.00013$ & $0.9824 \pm 0.00012$ & $2.033 \%$ \\
\hline 1 & DS & $\mathrm{Al} \mathrm{8 \%}$ & $7.1188 \pm 0.00169$ & $12.4173 \pm 0.00010$ & $0.9569 \pm 0.00006$ & $8.371 \%$ \\
\hline 1 & DS & $\mathrm{Al} \mathrm{4 \%}$ & $3.5878 \pm 0.00109$ & $6.2518 \pm 0.00007$ & $0.9586 \pm 0.00005$ & $4.208 \%$ \\
\hline 1 & US & C $1.5 \%$ & $3.1716 \pm 0.00104$ & $4.5234 \pm 0.00015$ & $0.9990 \pm 0.00016$ & $1.645 \%$ \\
\hline 2 & DS & $\mathrm{Al} 2 \%$ & $1.8637 \pm 0.00082$ & $3.2494 \pm 0.00010$ & $0.9646 \pm 0.00008$ & $2.173 \%$ \\
\hline 2 & $\mathrm{DS}$ & Al $8 \%$ & $7.1980 \pm 0.00143$ & $12.5669 \pm 0.00009$ & $0.9642 \pm 0.00007$ & $8.408 \%$ \\
\hline 2 & DS & $\mathrm{Al} 4 \%$ & $3.6828 \pm 0.00083$ & $6.4214 \pm 0.00010$ & $0.9642 \pm 0.00008$ & $4.296 \%$ \\
\hline 2 & DS & C $1.5 \%$ & $3.1876 \pm 0.00182$ & $4.5312 \pm 0.00012$ & $0.9991 \pm 0.00010$ & $1.648 \%$ \\
\hline 2 & DS & $\mathrm{Al} \mathrm{4 \%}$ & $3.6678 \pm 0.00568$ & $6.4232 \pm 0.00021$ & $0.9648 \pm 0.00012$ & $4.295 \%$ \\
\hline 2 & US & C $0.5 \%$ & $0.9973 \pm 0.00227$ & $1.0606 \pm 0.00012$ & $0.9729 \pm 0.00007$ & $0.397 \%$ \\
\hline $1-2$ & US & Pure Al & $1.0149 \pm 0.00045$ & $1.7318 \pm 0.00013$ & $0.9524 \pm 0.00007$ & $0.0117 \%$ \\
\hline $1-2$ & US & $\mathrm{Al} \mathrm{1 \%}$ & $0.8812 \pm 0.00055$ & $1.5243 \pm 0.00009$ & $0.9627 \pm 0.00005$ & $1.021 \%$ \\
\hline $1-2$ & US & $\mathrm{Al} \mathrm{4 \%}$ & $3.6030 \pm 0.00102$ & $6.2940 \pm 0.00010$ & $0.9599 \pm 0.00005$ & $4.229 \%$ \\
\hline $1-2$ & US & $\mathrm{Al} 2 \%$ & $1.7987 \pm 0.00070$ & $3.1359 \pm 0.00009$ & $0.9664 \pm 0.00005$ & $2.093 \%$ \\
\hline
\end{tabular}

Table 3.3: Measurements of thickness, area, and density of dummy targets most relevant to the aluminum target analysis [91].

A trio of lead antimony collimators was placed immediately after the target (lead antimony contains $95.5 \% \mathrm{~Pb}$ and $4.5 \% \mathrm{Sb}$ [67]). The first and third of these were cleanup collimators, while the second was the acceptance defining collimator. The first collimator was situated $74 \mathrm{~cm}$ downstream of the target; its apertures were 14 sided, forming an approximately 9x9 $\mathrm{mm}^{2}$ beam envelope allowing any electrons scattered along the target length to pass. A pair of retractable $5 \mathrm{~cm}$ thick tungsten shutters could be attached to the two horizontal octants for systematic background studies after Run 1.

The second downstream collimator defined the experimental acceptance, and was positioned 2.72 meters downstream of the target center. It was $15.0 \mathrm{~cm}$ thick, and apertures were 6 sided, defining the acceptance to about $400 \mathrm{~cm}^{2}$ in area per octant. This corresponded to an angular acceptance from the upstream end of the target of $\theta=5.8-10.2^{\circ}$, and $\theta=6.6-11.6^{\circ}$ from the downstream target face.

The third collimator was $11.2 \mathrm{~cm}$ thick and positioned 3.82 meters downstream of target center. It was positioned between a pair of aluminum plates for support, and provided several centimeters of clearance for the elastically scattered electrons in the desired kinematic range. After exiting this collimator, the scattered electrons enter the large toroidal magnet spectrometer, discussed in Section 3.7.

Lead lintels were installed between the magnet coils to shield the detectors from secondary scattered neutral backgrounds generated at the inner apertures of the defining collimator. Simulations showed that electrons from elastic and Møller scattering, when 
interacting with the defining collimator, generated photons having direct line-of-sight to the main detectors. Simulations also showed these lintels would reduce this neutral background by a factor of $90 \%$ [42]. Additionally, the lintels also provided shielding from an intense fountain of low-energy Møller scatters from the target [67].

The detectors were enclosed in a separate $122 \mathrm{~cm}$ thick concrete shielding hut. The upstream wall was $80 \mathrm{~cm}$ thick which effectively functioned as a fourth collimator. It was formed from 10 tightly fitting interlocking sections which were formed on the ground, and reassembled upright. The concrete was high density $\left(2700 \mathrm{~kg} / \mathrm{m}^{3}\right)$ barite-loaded concrete $\left(\mathrm{Ba}_{2} \mathrm{SO}_{4}\right)$ which was reinforced with stainless steel rebar.

The experiment was designed to minimize line-of-sight between the target and aluminum beampipe to reduce potential sources of background. Simulations showed this could be almost completely achieved with a water-cooled tungsten-copper plug beam collimator. This sat around the beam pipe like a $21 \mathrm{~cm}$ long donut, $7.9 \mathrm{~cm}$ in diameter, in the central aperture of the first collimator, $47 \mathrm{~cm}$ downstream of the target. The beam deposited approximately $1.6 \mathrm{~kW}$ of power in the plug, as determined from measured water flow and the temperature difference across it. The maximum angle passed by the beam collimator $\left(\theta_{\max }=0.88^{\circ}\right)$ corresponds to scattered events occurring at the downstream face of the target.

Figure 3.13 shows the various potential background sources and location of shielding with the main detector.

\subsection{Spectrometer magnet}

After leaving the third clean-up collimator the scattered electrons progress through the large $Q_{\text {weak }}$ toroidal magnet spectrometer (QTOR). It's design produces a large acceptance for elastic ep scatters and a high-degree of azimuthal symmetry. At the mean scattered electron angle of $7.9^{\circ}$, the $\int B \mathrm{~d} l$ is $0.9 \mathrm{~T}-\mathrm{m}$, which bends the negatively charged electrons an additional $14^{\circ}$, focuses them in $\theta$, and slightly de-focuses them in $\phi$. Low-momentum inelastic scatters are swept outside the detector acceptance while even lower-energy Møller scattering events are swept away at the upstream end of the magnet. Due to the $\phi$-dependence of the magnetic field profile, curvature of the elastic event envelope results in a mustache-shaped image on the focal plane [67].

The spectrometer consisted of eight identical resistive coils, each composed of a double pancake of 13 turns of copper conductor. Due to the iron-free nature of the magnet, hysteresis effects were not problematic and the field was highly reproducible. 


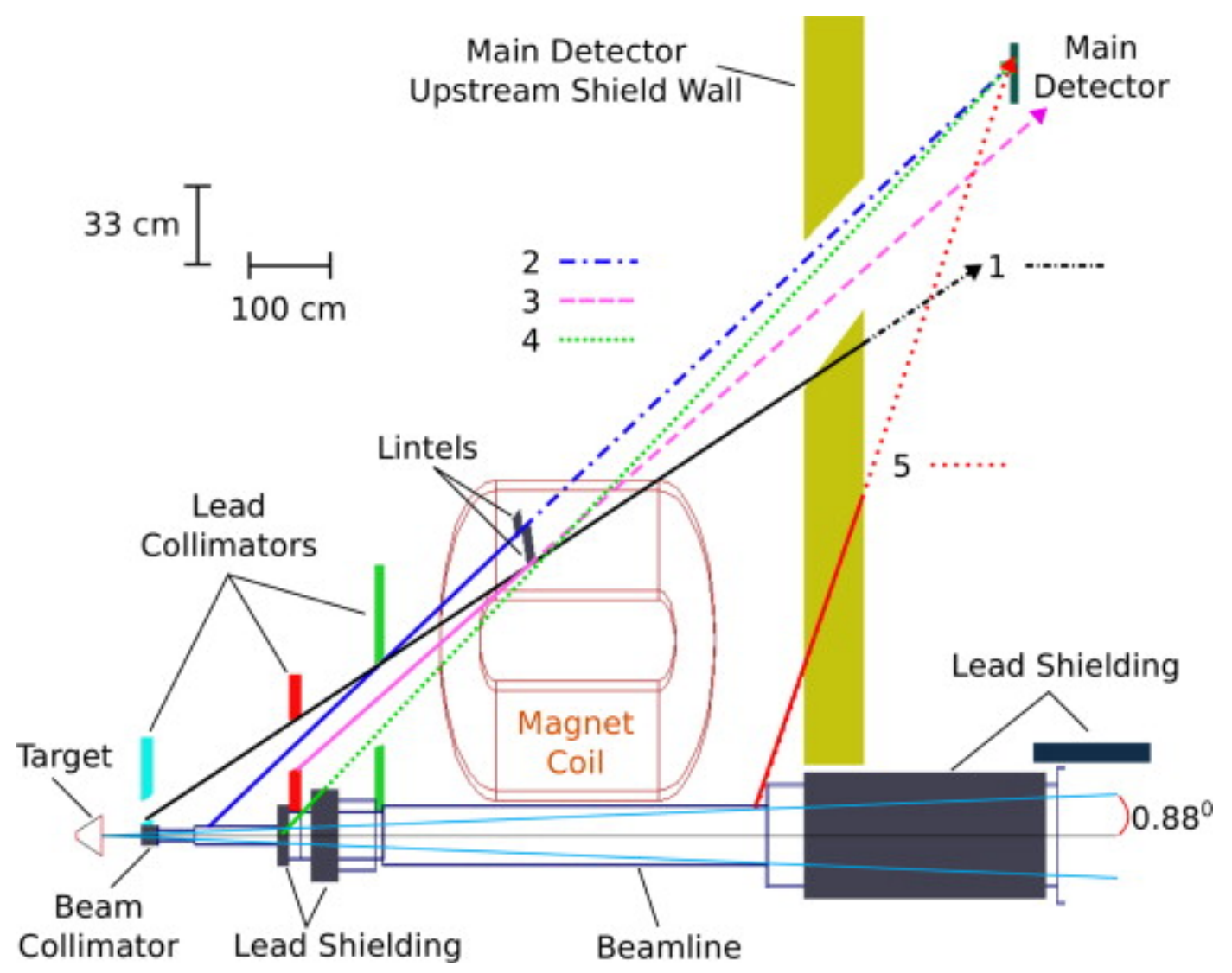

Figure 3.13: Cross-section of the experimental apparatus, showing the various sources of neutral background and corresponding main detector shielding. The vertical scale is amplified by a factor of three for clarity. Background type 1 (black line) shows that neutrals generated within the target are eliminated by the large detector shielding hut. Background types 2 and 3, represented by a dotted blue line or dashed pink line respectively, arise from scattering in the second collimator. Type 4, represented by a green dotted line, was discovered during experimental commissioning using dosimetry and trial shielding. Lead shielding was added to the beam pipe to reduce this. Any neutrals originating at the exit of the region just after the spectrometer magnet (type $5)$ were removed by the detector hut shielding. Reproduced from [67]. 
The current in the coils was monitored via a DC current transformer (DCCT) with a \pm 10 ppm current-regulating power supply [92]. A Hall probe was installed as an independent check on the power supply stability; it ultimately helped identify intermittent periods when radiation damage affected the DCCT stability.

By varying the QTOR current, and therefore the overall field and amount of magnetic deflection, various pieces of the scattering phase space could be mapped out. These QTOR current scans were an important diagnostic, as the amount of inelastic or Møller scattering could be directly measured. For instance, the ratio of inelastic scattering events from the $N \rightarrow \Delta$ transition is largest at the QTOR current setting of $6700 \mathrm{~A}$. Measuring rates at this kinematic setting is one method of accessing these backgrounds directly. Smaller scans around the elastic peak also proved crucial in understanding our acceptance and benchmarking our simulation.

\subsection{Detectors}

$Q_{\text {weak }}$ took data in two separate configurations: integrating or production mode, where beam currents were approximately $200 \mu \mathrm{A}$, and low-current or tracking mode. In production mode, the total measured scattering rate was over $6 \mathrm{GHz}$. As the scattering rate was too large to count individual particles, the signal itself was integrated. In tracking mode, beam currents were in the $50 \mathrm{pA} \mathrm{-} 1 \mu \mathrm{A}$ range, where individual scattering events could be reconstructed and scattering rates precisely measured.

Three primary detector arrays were used in production mode: eight Cherenkov main detectors measured the parity-violating asymmetry, two sets of luminosity monitors (upstream and downstream of the main detector array) were used to measure beam properties and backgrounds, and a focal plane scanner was used in one octant to compare the beam profile in both high- and low-current applications.

Figure 3.14 shows the global detector coordinate system, as seen from the target. Beam left is positive X. The coils numbered 2-9 are the QTOR coils. Each main detector also has a local coordinate system, which is useful during analysis, defined with the negative end as left and positive end as right (see Figure 3.14).

\subsubsection{Main detectors}

$Q_{\text {weak }}$ utilized an array of eight azimuthally symmetric main detectors. Each detector module was constructed of two $100 \mathrm{~cm} \times 18 \mathrm{~cm} \times 1.25 \mathrm{~cm}$ radiator bars, joined lengthwise for a total length of 2 meters. The bars were joined using a UV-transparent glue (SES-406). Each bar was made of Spectrosil 2000, an artificially fused silica material. 


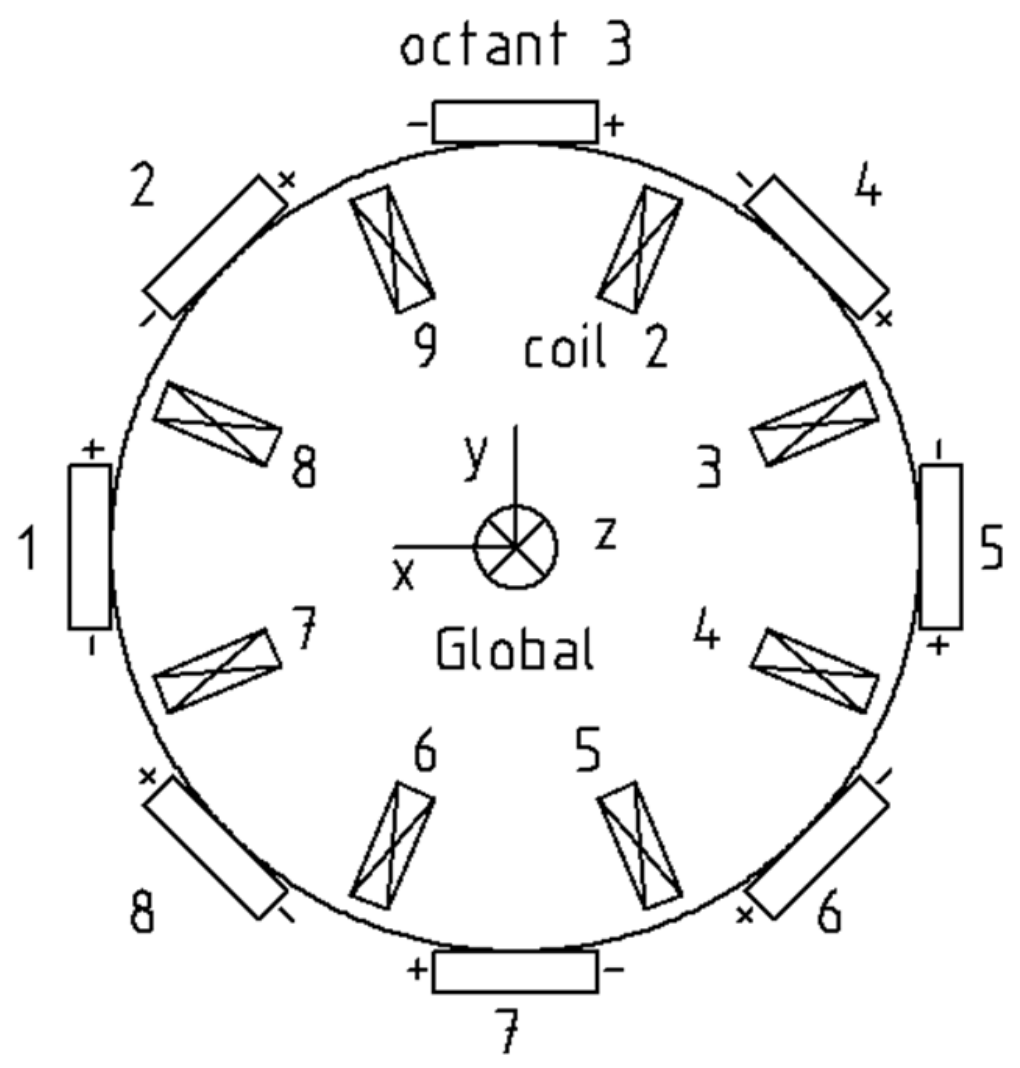

Figure 3.14: The global main detector coordinate system.

This was chosen for its particularly desirable qualities of radiation-hardness and low luminsesence, making them relatively insensitive to neutral backgrounds. Each surface was polished to $25 \AA$, with systematic point-to-point variations of $\pm 250 \mu \mathrm{m}$. Two photomultiplier tubes (PMTs) were also glued onto the end of each detector on the downstream facing side. The index of refraction of the quartz and glue was $n=1.482$ at a wavelength of $280 \mathrm{~nm}$. This corresponds to a Cherenkov cone angle of approximately $47.6^{\circ}$.

The eight detectors were arranged symmetrically about the beam axis at a radial distance of approximately $335 \mathrm{~cm}$. They were enclosed in light-tight box and supported on an aluminum structure known as the Ferris Wheel. Fine radial adjustments occurred after initial surveys to ensure a high-degree of azimuthal symmetry which is important to suppress parity-conserving processes. To suppress soft-neutral backgrounds and enhance our signal, $2 \mathrm{~cm}$ thick lead pre-radiators were installed on the front face of each bar. This increased the light yield by a factor of 7 and improved the signalto-background ratio by 20. However, shower fluctuations in the pre-radiator also introduced additional excess noise to the total asymmetry width $(\mathcal{O} 10 \%)$. 
Cherenkov light generated by scattered electrons traveled along the detector bar via total internal reflection and was collected at each end by the PMTs. Each event generated an average of 98 photoelectrons. The signals from each PMT were read out with two different types of custom bases: low-current, event-mode running used high-gain bases $\left(2 \times 10^{6}\right)$, whereas in production mode low-gain $(\sim 440)$ bases were used.

Figure 3.15 shows the lower-left quadrant of the Ferris Wheel and the focal plane scanner, discussed below in Section 3.8.2, in-situ. A full description of the main detector bars is outside the scope of this document; the interested reader is directed towards [93].

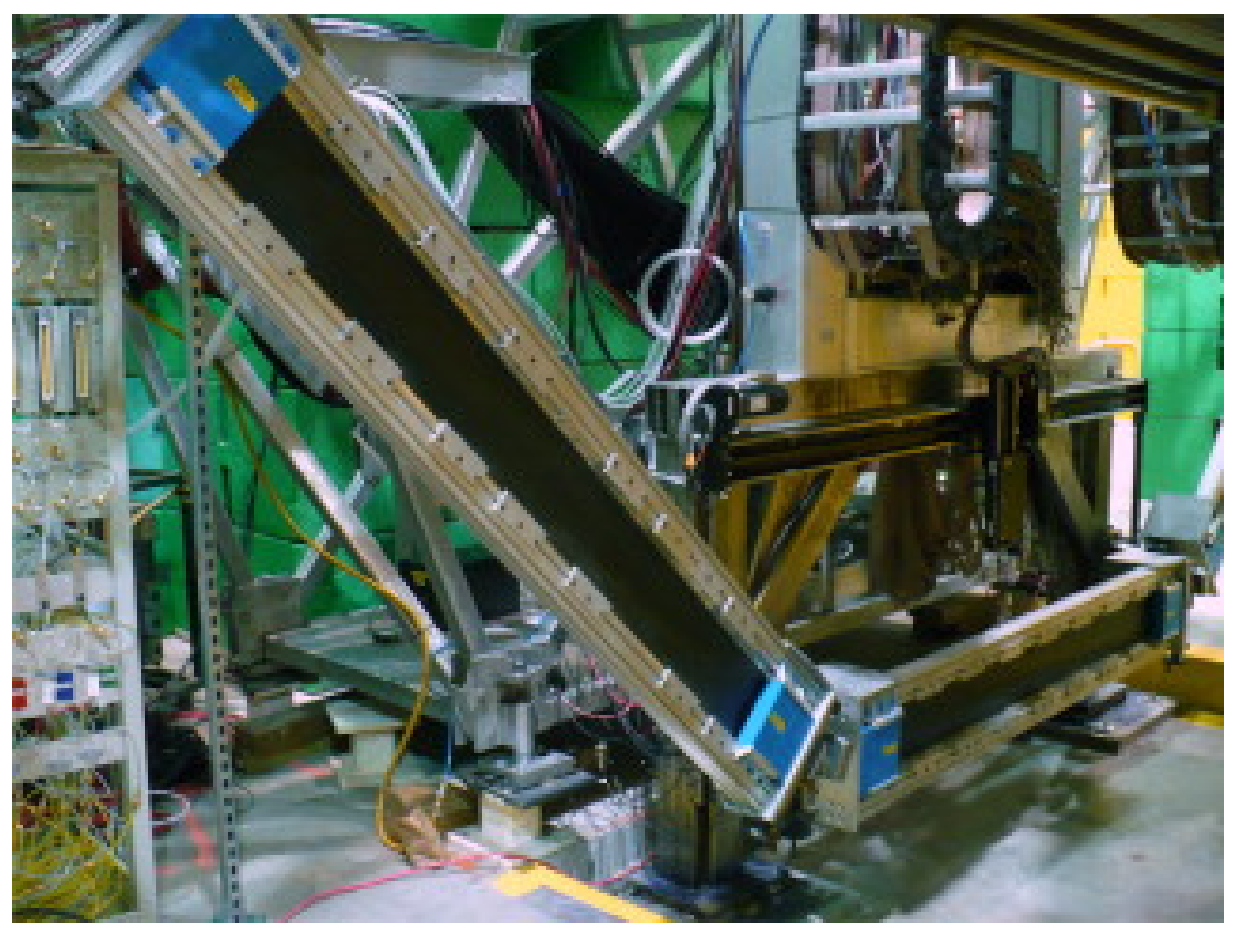

Figure 3.15: Photograph of main detector bars 7 and 8 in-situ (the lower-left quadrant of the Ferris Wheel). The pre-radiators are held in place by the aluminum frame, and the focal plane scanner is visible above the bottom-most detector (octant 7).

\subsubsection{Focal plane scanner}

The focal plane scanner was designed to measure the scattering profile at thg main detector bar over the full current range of the $Q_{\text {weak }}$ experiment (all 6 orders of magnitude between counting and production modes). The scanner consisted of two overlapping $1 \mathrm{~cm}^{3}$ cubes of synthetic quartz, which was the same material of the main detectors (Spectrosil 2000). The geometrical overlap of the two elements formed a small $1 \mathrm{~cm}^{2}$ area, with a coincidence condition between the two elements signaling a true event. A 
$2 \mathrm{D}$ motion system was implemented to move the scanner across the detector acceptance to map out the rate profile. This detector enabled experimental confirmation that the scattering profile on the main detector face matched that predicted by simulation, and was also was identical between low- to high-current running. As an example of this importance: if large target density fluctuations existed at high current this could have altered the scattering profile.

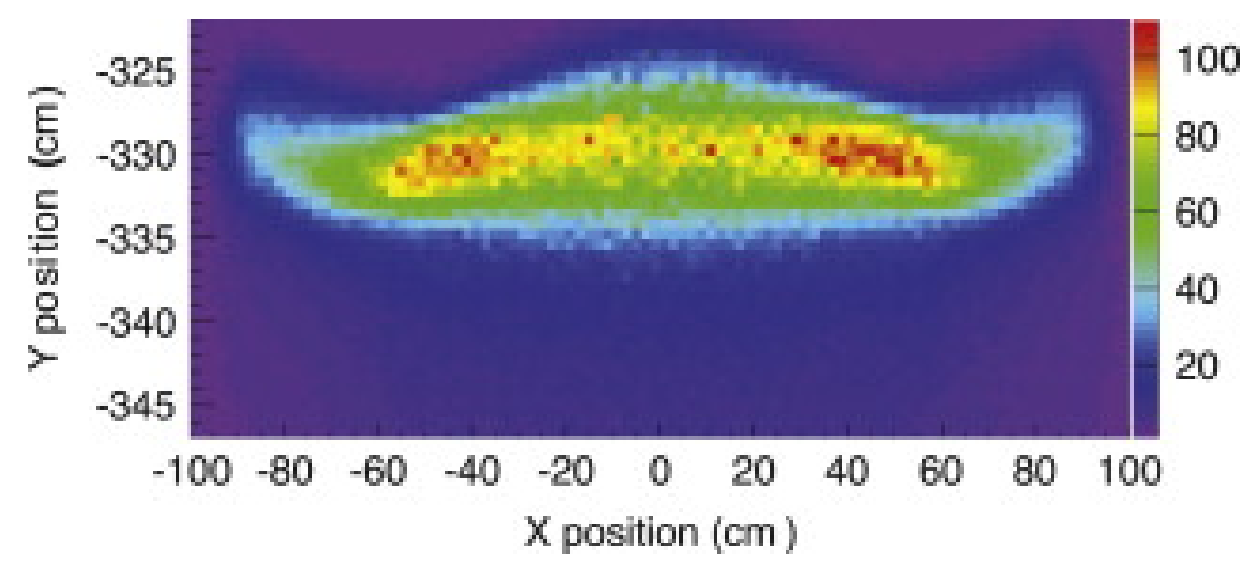

Figure 3.16: Scattered electron flux distribution in the bottom octant obtained by the focal plane scanner. The profile is a complex moustache shape, due to a combination of acceptance effects from the collimator system and fringe-field effects in QTOR. Reproduced from [67].

\subsubsection{Luminosity monitors}

Two sets of luminosity monitors were used as auxiliary detectors. Originally designed to monitor target density fluctuations, they were primarily used to study beamline background contributions. ${ }^{4}$ Four upstream luminosity monitors were placed on the upstream face of the primary collimator, where the approximate scattering angle was $5^{\circ}$. The high rates $(115 \mathrm{GHz})$ primarily came from low-energy Møller scattered electrons.

Eight downstream luminosity monitors were placed symmetrically around the beampipe, and were situated $17 \mathrm{~m}$ downstream of the target at an angle of $0.5^{\circ}$. Here, they were sensitive to similar rates of both Møller and Mott scattering events in the target. Although anticipated to see a smaller asymmetry than the main detectors due to the small $\theta$ and kinematics, the downstream luminosity monitors asymmetry widths were actually similar in size in practice.

Like the other detectors, both sets of luminosity monitors were constructed of radiation-hard Spectrosil 2000 quartz bars. They used a light-guide flushed with $\mathrm{N}_{2}$ gas to minimize corrosion. The upstream monitors had dimensions of $7 \mathrm{~cm} \mathrm{x} 27 \mathrm{~cm} \mathrm{x} 2$

\footnotetext{
${ }^{4}$ As discussed in Section 3.5.1, target boiling was minimal and well constrained.
} 
$\mathrm{cm}$, while the downstream monitors measured $4 \mathrm{~cm} \mathrm{x} 3 \mathrm{~cm} \times 1.3 \mathrm{~cm}$ with a $45^{\circ}$ taper at one edge. The downstream monitors had an additional $2 \mathrm{~cm}$ thick pre-radiator in-front to suppress low-energy backgrounds.

\subsection{Tracking system}

During $Q_{\text {weak }}$, dedicated periods of low-current running were done for systematic studies. These periods, known as tracking mode, were crucial to determining subtle-effects in $Q^{2}$ determination, analog response of the main Cherenkov detectors, and comparison with detailed simulations using both GEANT3 and GEANT4. Although the $Q^{2}$ acceptance is defined by the primary collimator, the light production in the main detectors are non-uniform, depending heavily on the event's location (kinematics) and that bar's individual response. This shifts the acceptance-weighted $Q^{2}$ by a few percent. Measurements produce an effective average- $Q^{2}$. Once simulations are properly benchmarked, they are used to determine the actual $Q^{2}$ at the interaction vertex. ${ }^{5}$

During these periods, which occurred every 1-2 months, individual scattering events were re-constructed via software and their $Q^{2}$ determined. The experimental tracking system consisted of 2 regions $^{6}$ : Region II consisted of two pairs of horizontal drift chambers (HDCs) upstream of the QTOR magnet, while Region III consisted of two pairs of vertical drift chambers (VDCs) and a pair of trigger scintillators after QTOR and immediately before the main detector array. Groups of these detectors are called a package (in Region III, a package consists of two VDC's and one trigger scintillator). HDC and VDC designs are distinguished by the dominant electron drift direction relative to the wire planes. In HDCs, the ionized electrons drift parallel to the wire planes, while in a VDC they drift perpendicular to them. The drift chambers are attached to arms which cover two octants $180^{\circ}$ apart. Being on separate arms, the Region II and III packages are free to rotate around the beam axis to cover each of the four octant pairs. One octant pair in each Region could be covered with either of the two sets of chambers for redundancy. When not in event-mode, the arms retract, pulling the packages out of the scattered electron envelope.

The HDCs established the scattered electron trajectory before QTOR, enabling one to track back to the target and establish the interaction vertex and scattering angle, $\theta$.

\footnotetext{
${ }^{5}$ An important related effect that the tracking system is involved in is determining small, subtle effects that occur when moving from the measured asymmetry at an average $Q^{2}$ to the average-asymmetry of a given $Q^{2}$ (that is, moving from $A\left(\left\langle Q^{2}\right\rangle\right)$ to $\left.\left\langle A\left(Q^{2}\right)\right\rangle\right)$.

${ }^{6}$ Region I originally consisted of gas electron multipliers (GEMs). However, due to problems in construction they were ultimately not installed.
} 
The VDCs, located downstream of QTOR, enabled tracking to the main detector. With knowledge of QTOR's magnetic field reconstruction of individual scattering events and their momenta is possible. By requiring various combinations of detector coincidences, detector efficiencies can also be studied.

\subsubsection{Horizontal drift chambers}

Five HDCs were constructed with the fifth one serving as a spare. One pair of HDCs constituted a Region II package, and the two packages were mounted on two arms, fixed $180^{\circ}$ apart. They were positioned between the $2^{\text {nd }}$ and $3^{\text {rd }}$ collimators upstream of the QTOR magnet. The arms were mounted on a central hub which rotated allowing coverage of all eight octants. The arms were positioned and retracted manually.

The drift chambers consist of two aluminum mylar cathode planes with an array of parallel sense and field wires, and uses a gas mixture of $65 \%$ Argon-35\% Ethane. When an electron passes through the chamber, the gas ionizes and the ionization electrons are pulled towards the grounded sense wires. Here the signal is amplified via an avalanche, where the analog signal is converted to a logic signal by an ECL amplifier discriminator card. The output signals are ultimately sent to time-to-digital converters (TDCs).

Each HDC consisted of six wire planes $\left(x, x^{\prime}, u, u^{\prime}, v, v^{\prime}\right)$, each with 32 sense wires and 33 field wires per plane. The sense wires were $20 \mu \mathrm{m}$ diameter gold-plated tungsten wires strung at a nominal tension of $20 \mathrm{~g}$, while the field wires were thicker $75 \mu \mathrm{m}$ goldplated beryllium-copper wires strung at a nominal tension of $30 \mathrm{~g}$. The wire pitch was $5.84 \mathrm{~mm}$, with spacing between planes at $19.0 \mathrm{~mm}$. The $u, v$ wires were at angles of $\pm 53.1^{\circ}$ relative to the $x$ wires to help offset left-right ambiguities of hits. The field planes were held at a potential of $-2150 \mathrm{~V}$; the sense wires were held at ground. The single wire position resolution was $\mathcal{O}(200 \mu \mathrm{m})$ and scattering angle resolution $\theta \sim 0.6$ mrad.

A full description of the HDCs included in [94].

\subsubsection{Vertical drift chambers}

Five VDCs were built with the fifth serving as a spare. Each chamber consisted of two anode wire planes held at ground, with each plane including 279 sense wires. These sense wires were $25 \mu \mathrm{m}$ gold-plated tungsten strung at a nominal tension of $60 \mathrm{~g}$. The wire pitch was $4.97 \mathrm{~mm}$, with planes oriented at angles of $\pm 26.56^{\circ}$ from the long axis of the chambers.

High voltage cathode planes were situated $12.7 \mathrm{~mm}$ above and below each wire 
plane. These operated at $\sim 3800 \mathrm{~V}$. The outer cathode planes were $12.7 \mu \mathrm{m}$ thick Mylar foils, aluminized on one side. The middle cathode was the same material but aluminized on both sides.

Due to the high number of wires in the VDCs $(279$ wires/plane $\times 8$ planes $=2,232$ wires), it became expensive to read out individual wire signals. Instead, a novel digital delay multiplexing system was developed. Every $8^{t h}$ plane-wired was ganged together, forming groups of 141 wires total. The wires of each gang were split into two components (left and right signals), with a $1.3 \mathrm{~ns}$ digital delay in-between wires. The outputs of each gang were input into a time-to-digital converter. Time differences between the left and right outputs would produce distinct peaks for each wire, enabling precise timing determination with a factor of 9 reduction in necessary read-out channels.

A full description of the VDCs are the subject of [95], while a detailed description of the multiplexing is the focus of [96].

\subsubsection{Trigger scintillators}

Plastic scintllators were placed immediately behind (downstream) of the VDCs to provide fast timing triggers during event-mode read-out. They were also used to study neutral backgrounds in the detectors.

The trigger scintillators were approximately $218 \mathrm{~cm} \times 30 \mathrm{~cm} \times 1 \mathrm{~cm}$ and made of Bicron BC-408 plastic. UV-transparent lucite light guides were used to connect to photomultiplier tubes. The signals were discriminated with a CAEN N842 8-channel constant fraction discriminator in conjunction with a CAEN V706 16-channel hardware meantime module. The resolution of the trigger scintillator setup, including the meantime unit was $\sim 460$ ps. During $Q_{\text {weak }}$ the discriminator was configured with a large $(150 \mathrm{~ns})$ output width, which defined the minimum double pulse resolution for this detector.

For a complete description of the design of the trigger scintillator electronics, see [42].

\subsection{Data acquisition}

The data acquisition system (DAQ) was separate for high-current production and lowcurrent tracking modes. During production running, the signal from each detector's photomultiplier tube is integrated, digitized, and read out using specialized low-noise analog-to-digital converters [97]. The data trigger is the MPS signal, generated by the helicity control board. In tracking mode the trigger is configurable; it can be set to be 
any individual detector or group of detectors. For example, during event-mode studies (the topic of Chapter 5 and Appendix B) the trigger originates from either of the trigger scintillators. In other studies, main detectors, pairs of main detectors, or even clocks (to read scalers) may act as triggering sources.

The raw data collection is processed by the CEBAF Online Data Acquisition (CODA). CODA communicates with various readout controllers (ROCs), where the electronics signals are processed. During production running, the ROCs were read out during the $70 \mu$ s hold-off period at the beginning of the new helicity state. In event-mode running a prescale factor was used to limit the trigger rates to $1-2 \mathrm{kHZ}$ to minimize computer deadtime. Raw data was stored on the Jefferson Lab computer cluster (the ifarm), and each 6-minute data segment, called a runlet, was about 2 GB of data. Real time analysis was conducted using a combination of a $Q_{\text {weak }}$ specific analyzer and ROOT. After analysis, information such as raw detector yields, computer asymmetries, and regression results were stored in a MySQL database. 


\section{Chapter 4}

\section{The Møller Polarimeter Analysis}

WEAK REQUIRED DETERMINATION of the electron beam polarization to $1 \%$ precision. Quoting percent-level knowledge is a substantial claim, and requires substantial supporting arguments. In this chapter Møller polarimeter results are reported where sub-percent polarization was consistently achieved during $Q_{\text {weak }}$ 's Run 2. During Run 1 the ultimate systematic uncertainty achieved was slightly above $1 \%$, due to problems with one of the polarimeter's quadrupole magnets.

Since the Møller polarimeter was briefly introduced in Section 3.4.1, this chapter will provide a comprehensive treatment of its systematic uncertainties, including analysis of the insertable half-wave plate. One key finding during $Q_{\text {weak }}$ was that the newly installed Hall C Compton polarimeter provided excellent agreement with the Møller polarimeter; this analysis is found in Appendix A.

\subsection{Configuration during $Q_{\text {weak }}$}

The physical configuration of the polarimeter was changed prior to $Q_{\text {weak }}$ with installation of a third quadrupole magnet (labeled Q2 in Figure 4.1). This quadrupole magnet was unnecessary for $Q_{\text {weak }}$, and was installed to provide stronger focusing power in the $12 \mathrm{GeV}$ era. It was installed during $Q_{\text {weak }}$ commissioning to take advantage of the extensive beam line work to install the Compton polarimeter, and was left unpowered.

During Run 1 the Møller optics began exhibiting time-dependent behavior, and the beam operators noticed the beam downstream of the Møller target would drift substantially, requiring re-steering. Figure 4.2 shows the correlation of left and right main detector acceptance, as determined by the hodoscopes placed immediately before the detectors. Color shows the relative intensity. The combination of problems with beam steering and these tune plots suggested a problem in the Møller optics. A turnto-turn short in the large quadrupole coil packs was identified as the culprit. 


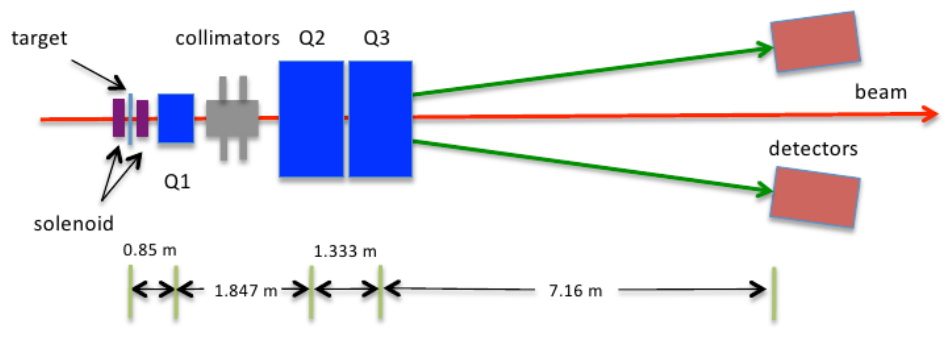

Figure 4.1: Schematic of the Hall C Møller polarimeter. The second quadrupole (Q2) was installed for the $12-\mathrm{GeV}$ upgrade and remained off during $Q_{\text {weak }}$. During $Q_{\text {weak }}$ Run 2, the Q3 was removed and Q2 was shifted downstream to take its place.
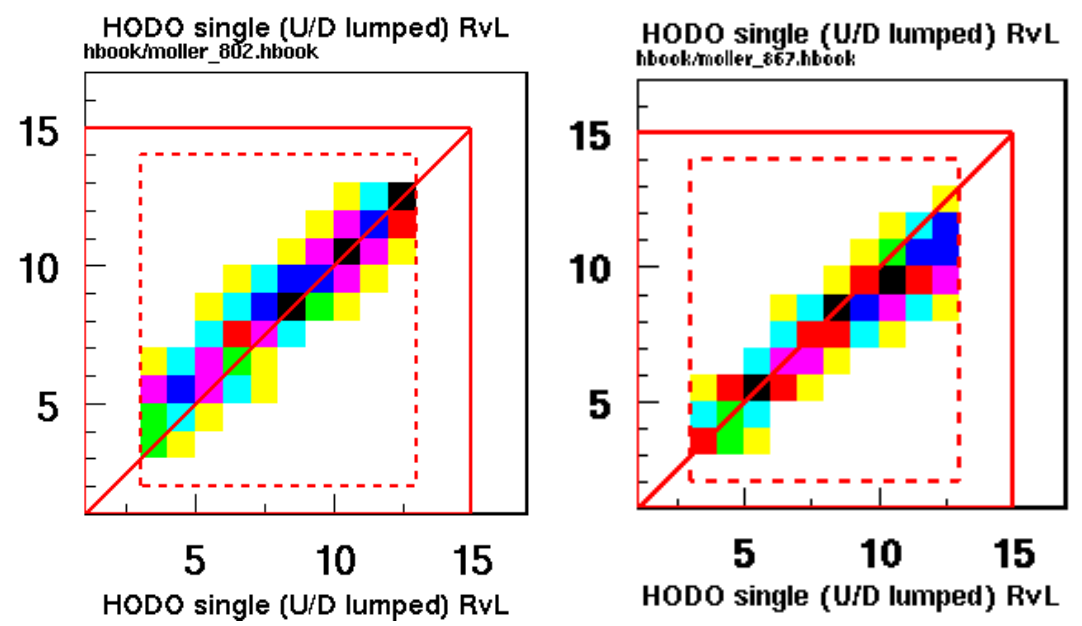

Figure 4.2: Correlations of right versus left Møller detector acceptance. (Left) A properly functioning optical system produces a tight correlation. (Right) Problems in the large quadrupole resulted in an asymmetric detector acceptance.

Near the end of Run 1, two Hall probes were placed inside Q3 along the beam line: one in both the upper beam-left and beam-right quadrants. Figure 4.4 shows the measured field decay of the beam right probe during a 6 hour period, after the quadrupole had been cycled and set to its nominal current (occurring at time $=0$ ). It clearly identifies Q3 as problematic. The quadrupole consists of 4 coil packs. Each coil assembly consists of 3 pancake windings, creating a magnetic field proportional to the current. Between each pancake is a small gap of insulating material. This appears to have failed during $Q_{\text {weak }}$ Run 1, creating a short in one coil between two pancakes. This quadrupole short can be viewed as either a reduction in the number of coils or the effective current in each pancake. Figure 4.3 shows a photograph of the quadrupole disassembled and beam line removed, looking upstream. The arrow indicates one quadrant's coil packs, and the "R" and "L" specify the approximate location of the beam-right and beam-left hall probes, respectively. 


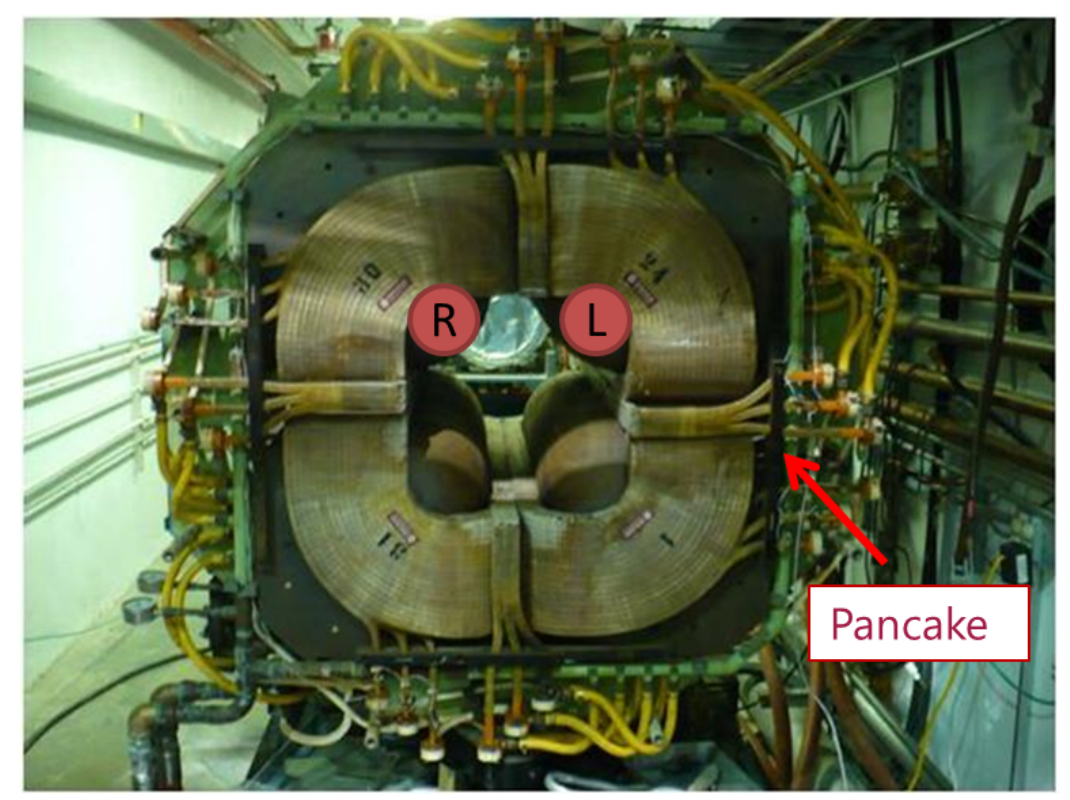

Figure 4.3: Photograph of large Møller quadrupole in-situ. The quadrupole has been disassembled and the beam line was removed. The photograph is looking upstream.

During the planned 6-month down between Run 1 and Run 2, Q3 was removed. Q2 was tested, and found functional enough for the remainder of the $Q_{\text {weak }}$ experiment. Q2 was then shifted downstream and relabeled Q3 to avoid confusion.

The Q3 coil short resulted in systematic shifts in analyzing power, as shown in Figure 4.5. For Run 1, these shifts were assessed on a measurement-by-measurement basis. For Run 2, the device's analyzing power was stable, but slightly shifted from the Run 1 value due to slight changes in location of the detectors and a properly functioning magnet. The effect of the coil short was studied through careful modeling with magneto-static generators. These modeled the magnetic field starting with the currents flowing through four coils, and with one coil's current being reduced relative to the others. These were then fed into the Møller Monte Carlo. The specific results are discussed later in Section 4.2.6.

The Møller Monte Carlo is a simple aperture checking tool, not a sophisticated GEANT-like comprehensive simulation. It determines the path of both the scattered and the recoil electron after scattering, determining the likelihood of a detected coincidence. It includes all the optical (magnetic) elements, an enclosure of movable collimators to reduce Mott scattering, and two lead immovable collimators positioned immediately in front of the detector array. It also includes multiple scattering, radiative effects, and corrections for the atomic fermi motion of the target electrons (the Levchuk effect). The Monte Carlo is benchmarked against one of the most sensitive experimental 
Møller Relative Quad Field (May 6, 2011)

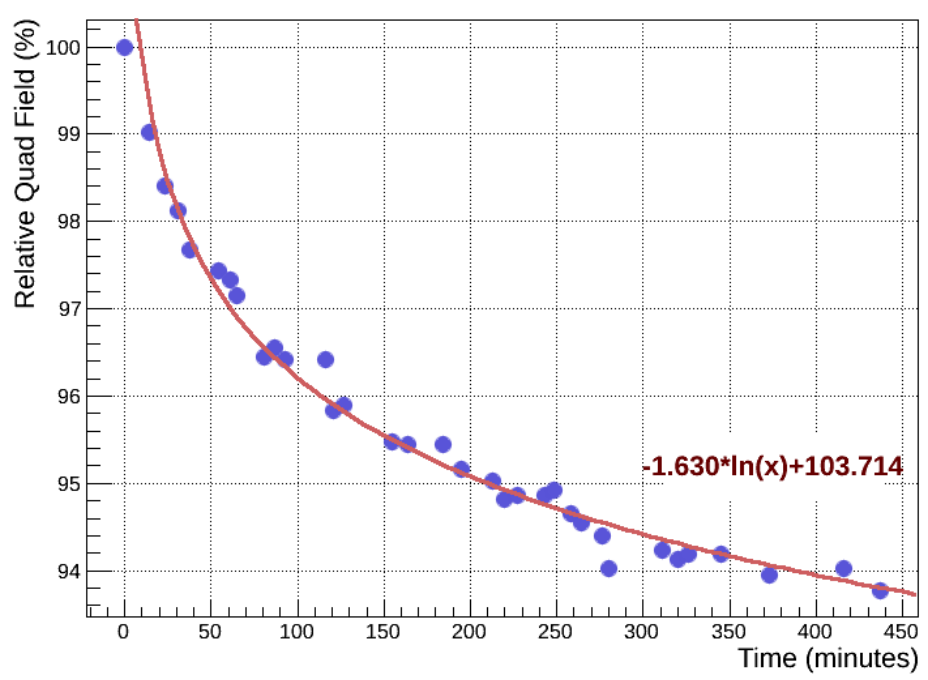

Figure 4.4: Relative quadrupole field versus time as measured with a Hall probe during the $Q_{\text {weak }}$ Run 1 beam position scan. Most production measurements took an average of 1 hour of data, where the field decays fastest. The logarithmic fit helps guide the eye.

systematics: the variation of the polarimeter performance with beam position on target. In an effort to improve agreement with this empirical study, the optical transport algorithms were modified. These improvements included:

- Augmenting the solenoid transport algorithm to incorporate only the solenoid exiting field for the scattered particles. The initial routine implemented the full solenoid transport, even though the exiting electrons only experienced the second half of the field. This had a significant impact on the overall detector acceptance and analyzing power.

- Implementing $2^{\text {nd }}$ order quadrupole TRANSPORT matrices [98, 99]. The previous routine assumed a thin-lens approximation. This improvement did not have a large impact on the acceptance or analyzing power.

- For Run 1, implementing a magnetostatic field map generated by POISSON [100]. This enabled modeling of the impaired quadrupole.

- Rotating the detectors through a small angle to be perpendicular to the electron paths. This was incorrectly implemented in the original Monte Carlo.

These improvements, as well as several others in the analysis implemented by the author, are outside the scope of this document. Interested readers are referred elsewhere for a more detailed presentation $[101,86]$. 


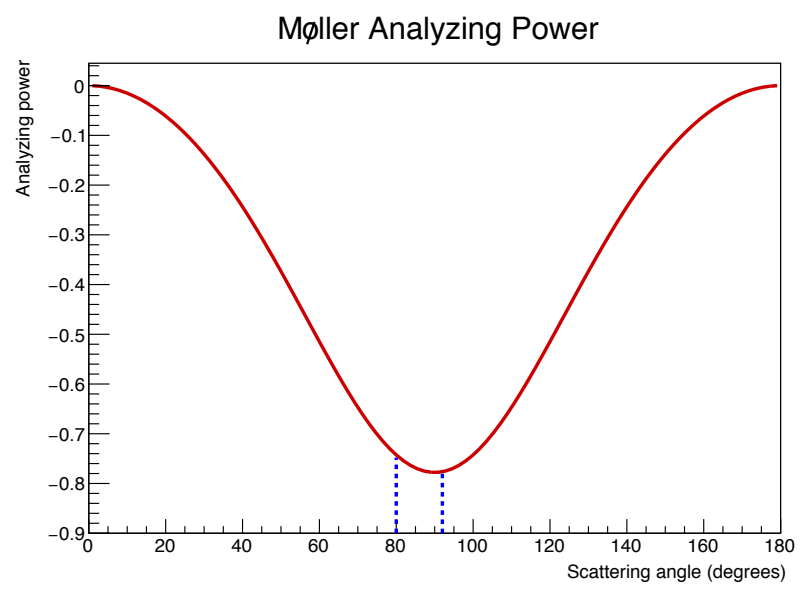

Figure 4.5: Møller analyzing power versus center-of-mass scattering angle for a system with broken optical transport. Compared to the ideal case (Figure 3.6), the blue lines show an asymmetric acceptance for a broken quadrupole. The effect is greatly exaggerated here as an illustration.

\subsection{Analyzing power determination and uncertainty}

Møller polarimetry is conceptually and experimentally relatively simple and fast due to the fortuitous combination of large cross section and analyzing power. From equation (3.7), reproduced here, Møller polarimeters exploit the cross section's helicity dependence in doubly polarized electron-electron scattering:

$$
A_{\text {exp }}=\frac{N^{+}-N^{-}}{N^{+}+N^{-}}=\left|P_{Z}^{T}\right|\left|P_{Z}^{B}\right| A_{Z Z}(\theta)
$$

where $A_{\exp }$ is the parity-conserving asymmetry, $N^{+}\left(N^{-}\right)$is the number of scattering events from a beam of positive (negative) helicity, $P_{Z}^{T}$ is the target polarization, $P_{Z}^{B}$ is the longitudinal polarization of the electron beam, and $A_{Z Z}(\theta)$ is the analyzing power. Therefore, to accurately determine $P_{Z}^{B}$, one must confidently determine the target polarization and analyzing power, and precisely measure $A_{\text {exp }}$.

The target polarization is determined from the well-known magnetic properties of pure iron. Some assumptions must be made to equate the bulk magnetic properties with electron polarization, but these are believed to be under sufficient theoretical control [85]. Additional uncertainties in target polarization come from impurities, any slight misalignments of the foil relative to the magnetic field, and beam-induced target heating. Discussions of the target polarization are found in Section 4.2.1, below, while calculations of depolarization due to target heating are described in Section 4.2.2.

The calculated analyzing power incorporates magneto-optical tuning and the device acceptance. It also accounts for other effects, such as multiple scattering, external bremsstrahlung and atomic Fermi motion (the Levchuk effect [102]). It is benchmarked 
against several systematic studies, in particular those which studied the rate variation with beam position on target compared to the measured variation.

In this section, the foil magnetization and system analyzing power are presented. A treatment of the relevant systematic uncertainties is also provided. The systematic uncertainty is split into two components: point-to-point uncertainties, and those of scale or normalization. Point-to-point uncertainties are those that are particular for that day's measurement, such as the measurement's statistical contribution or beam position on target. The normalization uncertainties are correlated between all the points, and can be represented as an uncertainty band, which shifts the overall normalized mean value.

\subsubsection{Nominal target polarization}

The nominal target (electron) polarization is known from the spin properties of magnetically saturated iron. For a general atom, the polarization and magnetization is given by the relation

$$
P_{t}=\frac{M_{s}}{\mu N_{e}},
$$

where $M_{s}$ is the contribution from the electron spin in the iron atom, $\mu$ is the electron's magnetic moment, and $N_{e}$ is the number of electrons in the atom. For iron, $N_{e}=26$. To determine $M_{s}$ one must determine the total magnetic properties of the system and then remove the orbital contribution. With an externally applied 4T magnetic field and the target foil oriented at $90^{\circ}$ relative to the field, the nominal target polarization is $(8.043 \pm 0.015) \%$ at room temperature $(T=294 \mathrm{~K})$ [103].

Two problems with this nominal calculation were discovered recently. First, the calculation of the orbital contribution [104] used the classical value for the electron's anomalous magnetic moment, $g=2$, instead of the correct value of $g=2.0023$. Second, the same incorrect value was used again in the calculation of target polarization, leading to an overall decrease in nominal target polarization by $0.24 \%$ to $(8.024 \pm 0.015) \%$.

Several other corrections to $M_{s}$ are required. During $Q_{\text {weak }}$, the Møller solenoid was run at $3.5 \mathrm{~T}$ rather than $4 \mathrm{~T}$, reducing the relative target polarization by $0.05 \%$ [105]. In addition, the foil was $99.85 \% \mathrm{Fe}$, with the largest contaminants coming from Co, $\mathrm{Cu}$, and $\mathrm{Ni}$ (400 ppm each). While all three contaminants provide polarized electrons to some degree, we conservatively assume these provide a pure dilution, and assign a $100 \%$ uncertainty. The final target polarization is $(8.008 \pm 0.019) \%[106]^{1}$.

\footnotetext{
${ }^{1}$ The saturation magnetization of iron at $0 \mathrm{~K}$ is $2.2160 \mu_{B}$. In the literature this fact is used to claim that only two of the four valence electrons are polarized. This statement is misleading, as once an
} 


\subsubsection{Target temperature}

As the target foil heats up due to the beam, the Møller iron target de-magnetizes slightly. Typically this effect is small at the 1-1.5 $\mu \mathrm{A}$ currents used for Møller measurements, but can be several percent at even the modest current of $5 \mu \mathrm{A}$. Fortunately, the temperature dependence of bulk iron's magnetic properties is well known $[105,107,108,109]$ and can be corrected for. Determining the target temperature rise, and therefore the de-polarization, consists of a two-step process. First, the temperature rise of the target for different beam currents was calculated numerically. Second, the actual demagnetization was determined using a fit to published $M$ vs. $T$ data. To be conservative, the uncertainty of this calculation is taken as $100 \%$ of the size of the effect.

To calculate the temperature of the target, I followed the general prescription of [110], which starts with solving the one-dimensional differential heat equation

$$
\frac{1}{r} \frac{d}{d r}\left(r \lambda \frac{d T}{d r}\right)=-Q I_{e} \phi(r)
$$

where $Q$ is the energy deposition for an iron target $(1500 \mathrm{~W} / \mu \mathrm{Am})$ assuming $d E / d x=$ $2 \frac{\mathrm{MeV}}{\mathrm{gcm}^{2}}, I_{e}$ is the electron beam current in $\mu A$, and $\lambda$ is the thermal conductivity $(\mathrm{W} / \mathrm{cm}$. $k$ ) of iron. $\phi(r)$ denotes the (normalized) radial profile of the electron beam. In [110] $\lambda$ is assumed constant. However, in reality $\lambda$ is a function of temperature $(\lambda \equiv \lambda(T))$, and the temperature of the foil is location dependent $(T \equiv T(r))$. Ultimately the thermal conductivity can be written as a function of radius $(\lambda \equiv \lambda(T(r)))$. The full solution is

$$
\Delta T=-\frac{Q I_{e}}{2 \pi} \int_{R}^{0} \frac{1-\mathrm{e}^{-\frac{1}{2}\left(\frac{r^{\prime}}{\sigma}\right)^{2}}}{\lambda\left(r^{\prime}\right) r^{\prime}} d r^{\prime} .
$$

Here, we assume a Gaussian shaped beam of width $\sigma$ and radius $R$. Several harp scans indicated the beam size to be at most $\sigma=100 \mu \mathrm{m}$ [106]. The thermal conductivity is approximately linear with $T$ over the range of interest [111], allowing one to numerically integrate over $R$ to determine the foil's total change in temperature, $\Delta T$ (the target edge is assumed to be at room temperature $(291 K))$. Table 4.1 gives the total calculated temperature rise at the target center for multiple beam spot sizes, assuming a beam current of $2 \mu \mathrm{A}$.

After computing the temperature, the magnetization is directly calculable [103, 105] using

$$
M_{s}(T)=M_{0}(T=0 K)\left(1-a_{3 / 2} T^{3 / 2}\right),
$$

atom is part of a solid lattice, the original atomic orbitals are significantly changed and naive counting of electron pairing is no longer appropriate. The fact that the magnetization is near an integer value is pure coincidence. 


\begin{tabular}{cc} 
Beam spot width $(\mu m)$ & $\Delta T\left({ }^{\circ} C\right)$ \\
\hline 30 & 17.15 \\
60 & 15.06 \\
70 & 14.64 \\
80 & 14.24 \\
90 & 13.89 \\
100 & 13.57
\end{tabular}

Table 4.1: Calculated temperature rise at Møller target center for various beam spot sizes at a beam current of $2 \mu \mathrm{A}$.

\begin{tabular}{cc}
$T(T)$ & $M_{s}(T) / M_{s}(291 K)$ \\
\hline 291 & 1.0000 \\
294 & 1.0000 \\
306 & 0.9986 \\
321 & 0.9971
\end{tabular}

Table 4.2: Relative demagnetization between the Møller target at temperature $T$ and $T=291 K$ (room temperature).

where $a_{3 / 2}$ is a constant $\left.\left(a_{3 / 2}=(3.61 \pm 0.07) \times 10^{-6} \text { (degrees }\right)^{-3 / 2}\right)$ and $M_{0}(T=0 K)=$ $2.216 \mu_{B}$. Table 4.2 gives the relative magnetization (and therefore depolarization) between a heated target and a target at room temperature. For $Q_{\text {weak }}$, the final correction value is taken as $(0.14 \pm 0.14) \%$.

\subsubsection{Solenoid field uncertainty}

One important systematic check is to study the measured polarization's dependence on the solenoid field. Magnetic saturation in iron occurs at $2.2 \mathrm{~T}$; as the solenoid field increases the measured polarization increases until $2.2 \mathrm{~T}$, whereafter the measured polarization is then constant. Measurements are usually run at $3.5 \mathrm{~T}$, ensuring almost total magnetic saturation. However, any foil tilt or warping will cause small, subpercent changes to the magnetization and analyzing power, as the electrons will have an in-plane magnetization component [103]. In total, the solenoid contributes to the overall uncertainty in four distinct ways:

- Target foil tilt relative to $\mathbf{B}$-field direction

- B-field strength

- Solenoid position

- Solenoid focusing

Figure 4.6 shows the calculated magnetization dependence for various foil angle tilts (i.e. B-field direction). Note that for slightly misaligned foils the measured value 

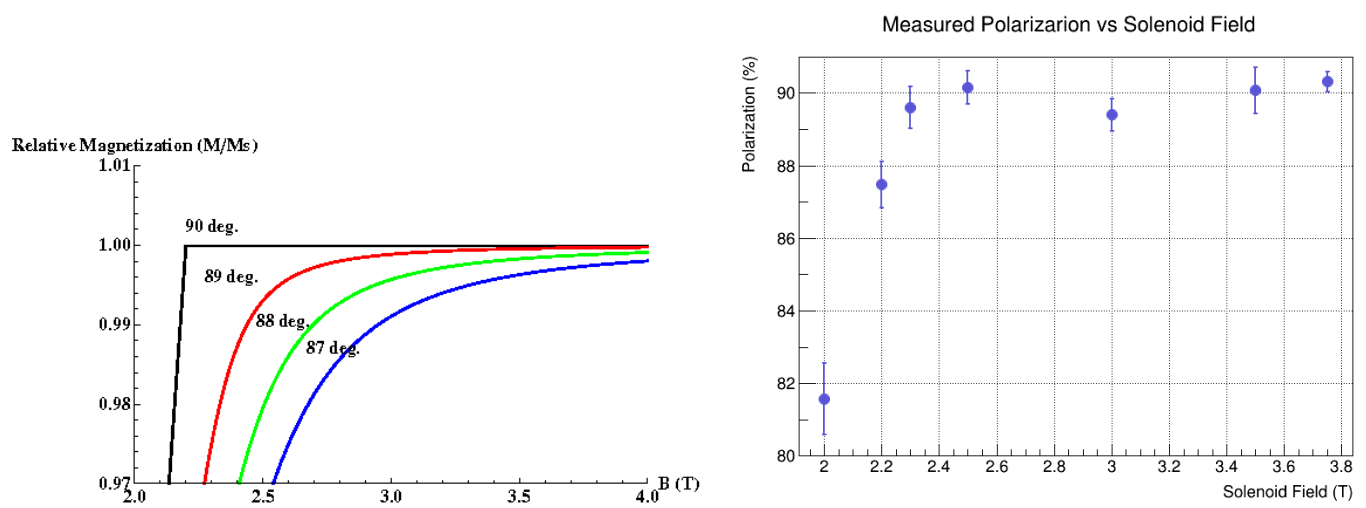

Figure 4.6: Left: Calculated magnetization dependence on field for pure iron, shown for multiple foil tilts (in degrees). Note that for a perfectly perpendicular foil, the magnetization rises sharply at exactly $2.2 \mathrm{~T}$. Taken from [86]. Right: The measured results from $Q_{\text {weak }}$ Run 1. Taken from [112].

plateaus less quickly, even to a lower value. Our measured polarization dependence is also shown and appears consistent with less than a $2^{\circ}$ misalignment of the foil relative to the solenoid field. No correction is made accounting for this slight offset; instead the uncertainty is conservatively taken as the upper limit for a $2^{\circ}$ tilt.

The transverse position of the solenoid itself was studied, leading to a modest increase in analyzing power uncertainty. To become superconducting, the solenoid magnet is cooled with cryogenic Helium and operates at $\sim 4 K$. As the magnet cools from room temperature, the solenoid coils contract slightly and shift. This movement is reproducible at the $0.5 \mathrm{~mm}$ level, causing small changes in the axial field center, affecting the scattered electron path slightly. A simulation-based study was done to determine the dependence on this degree of freedom. Figure 4.7 shows that shifting the simulated solenoid position results in slight position-dependent rate adjustments. The uncertainty contribution from solenoid position is $0.23 \%$, and is assumed evenly split between point-to-point and correlated normalization types.

Both the solenoid $\mathbf{B}$-field strength and focusing uncertainties were determined by simulation. The B-field, or solenoid strength, was varied by $5 \%$ to determine a $0.03 \%$ effect on the calculated analyzing power. Likewise, the overall effect of the solenoid (i.e. its focusing) was determined via simulation by turning off the solenoid field completely. The overall effect was $0.21 \%$ on the analyzing power.

\subsubsection{Quadrupole currents}

Previous Hall C experiments assumed the individual quadrupole fields (currents) were completely uncorrelated. This is overly conservative, as they are strongly correlated. 


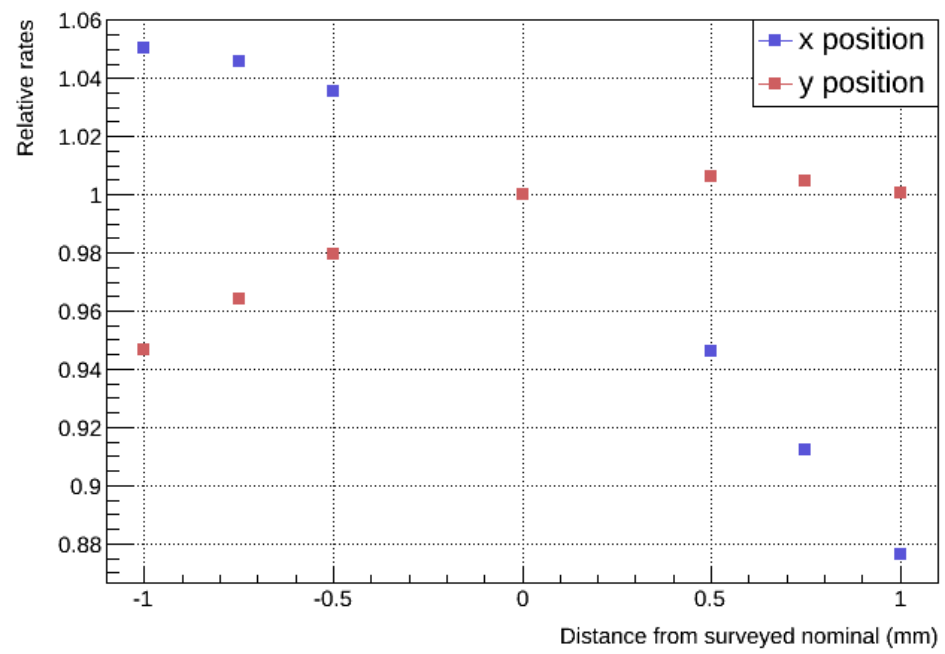

Figure 4.7: Relative rates from simulation as a function of absolute solenoid position. A shift in the solenoid $x$ direction of $0.5 \mathrm{~mm}$ increases the rates at target center by about $\approx 4 \%$. Although the solenoid placement was accurately measured by survey, a slight offset of $-0.5 \mathrm{~mm}$ was introduced in the simulation to improve agreement with the position scan.

Whereas one quadrupole focuses in one direction and de-focuses in the transverse direction, a system of two quadrupoles in series act as an optical lens, enabling precise focusing power in both directions. A change in field strength in one quadrupole would shift the focus, but a corresponding shift in the other magnet can re-align it. This relationship was modeled during design of the polarimeter [103], but has never been experimentally measured. During $Q_{\text {weak }}$, this was explicitly demonstrated to understand its effect on analyzing power and reduce the overall uncertainty contribution.

The quadrupole strength systematic study was performed in December 2010. The nominal quadrupole settings were typically 93.7 A (Q1) and 129 A (Q3). It was assumed we knew the quadrupole currents to within $2 \%$ of themselves. For the study, each quadrupole current was individually shifted up or down by its respective uncertainty. Then, after observing a shift in the optical tune plot, the other quadrupole was changed to re-align to a nominal optical tune. Figure 4.8 provides examples of the tune plots in this study.

The study was also simulated and showed qualitative agreement with the measured data. These simulations showed a $1 \%$ offset in the Q1 operating current could be compensated with by changing the Q3 current, and doing so would shift the inverse analyzing power $0.075 \%$, from 15.615 (nominal) to 15.627. A similar effect could be seen for a $3 \%$ offset in Q3, although the effect was smaller (0.05\%) In all, accounting for the quadrupole correlation drops the contributed systematic uncertainty (dA/A) from 

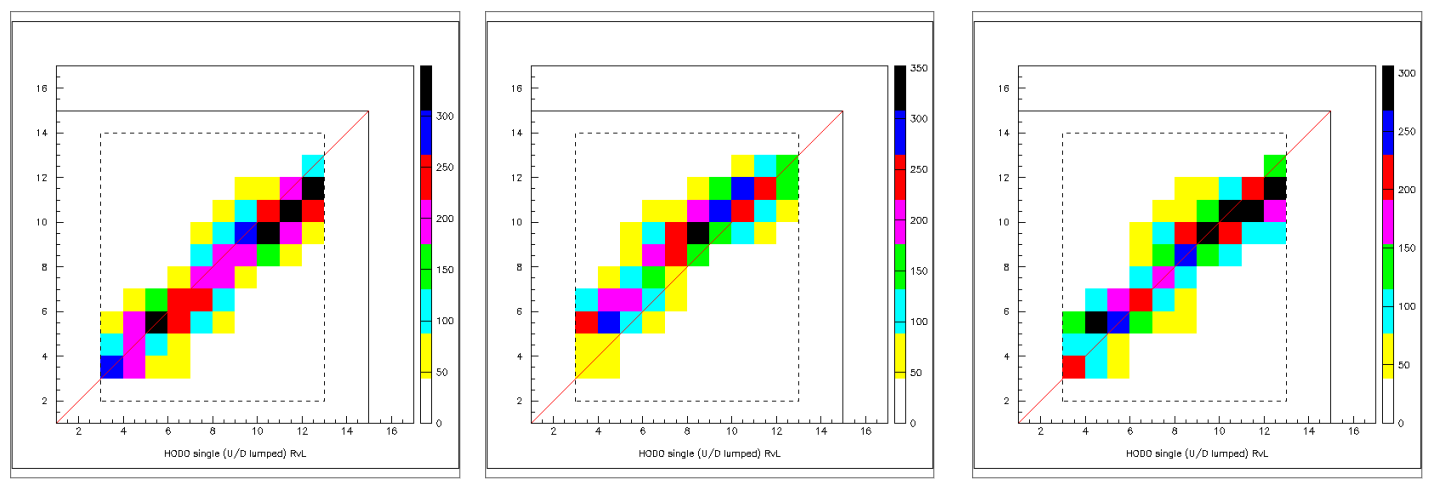

Figure 4.8: Histograms of right versus left hodoscope arrays; otherwise known as tune plots. (Left) A nominal tune. The red line gives the ideal detector correlation. (Center) A shift in the Q3 3\% field shifts the tune unless compensated by Q1 (Right).

\section{$0.45 \%$ to $0.085 \%$.}

One extra effect has recently been discovered. The small quadrupole (Q1) magnetic field was measured and found to be $8 \%$ smaller than originally thought. Preliminary studies show this effect does not change the analyzing power in a fully functioning twoquadrupole system, such as in Run 2, because of the quadruple correlations discussed. However, this does have a large potential effect for the Run 1 results when Q3 exhibited the coil short discussed in Section 4.1. Investigations of this potential problem in Run 1 is on-going.

\subsubsection{Position and angle systematic uncertainty}

Position and angle sensitivity of the electron beam on target are one of the Møller device's largest systematic uncertainty contributions ${ }^{2}$. During measurements, care was taken to minimize position and angle drifts, and measurements with drifts or target positions larger than $0.5 \mathrm{~mm}$ away from the target center were discarded. The accepted measurements were corrected for the actual beam position and angle measured.

As we correct for the position dependence, the position uncertainty is based on how well we know that correction. To make this correction, we need to understand three basic things:

- The dependence of the measured polarization on beam position $(\partial P / \partial x)$

- The uncertainty of projecting from the BPMs to the target $\left(\Delta P_{\text {calc }}\right)$

- The instrumental uncertainty in absolute BPM position $\left(\Delta P_{\text {instr }}\right)$

\footnotetext{
${ }^{2}$ Normally this isn't the case, but $Q_{\text {weak }}$ 's low beam energy results in sampling a larger phase space where the analyzing power changes more rapidly.
} 

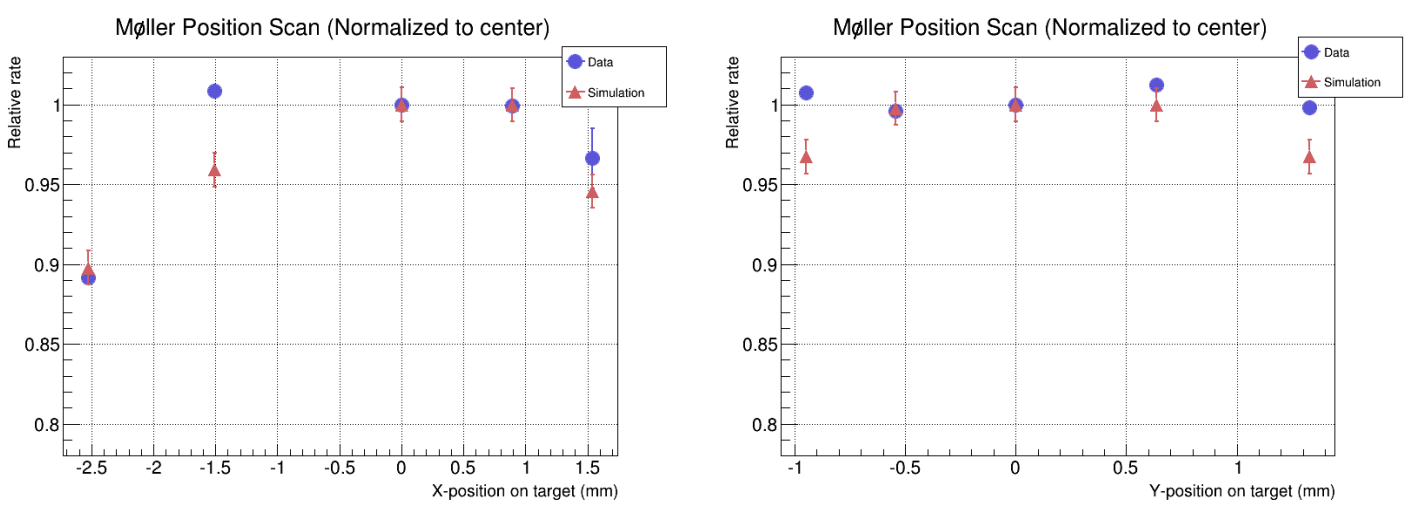

Figure 4.9: Position sensitivity results for Run 2. Scans were done in both $x$ and $y$ directions (left and right, respectively). The decent agreement between simulation and measured rates increases confidence in the simulation.
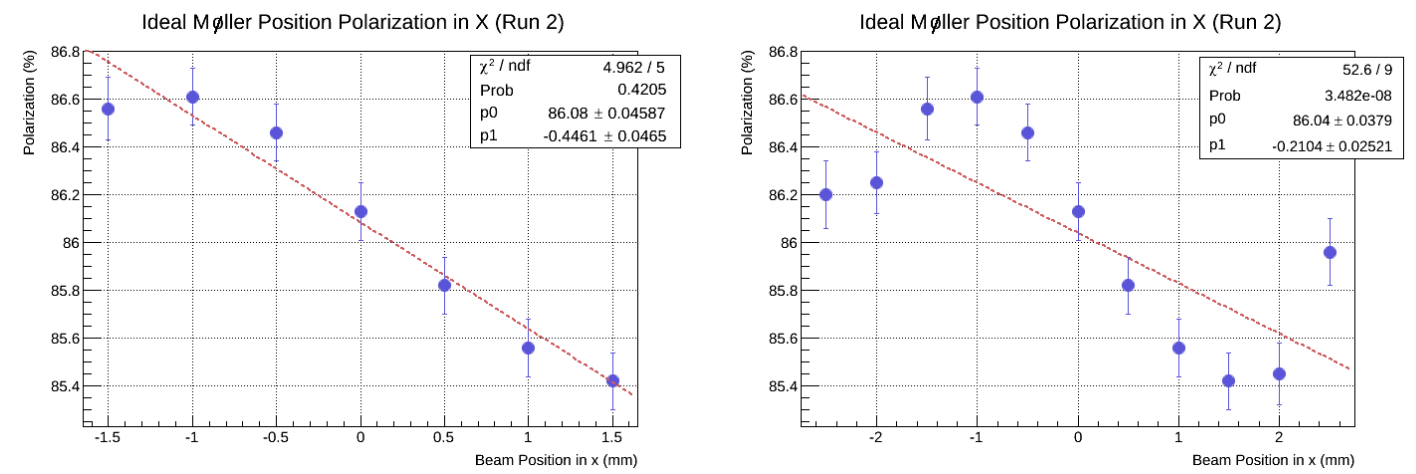

Figure 4.10: Simulated polarization dependence on target $x$-position for two different ranges in $x$-position. (Left) The fit using only data of $|x|<=1.5 \mathrm{~mm}$. (Right) Fits using data out to $|x|<=2.5 \mathrm{~mm}$ are poor.

where $x=x, y, x^{\prime}, y^{\prime}$. The dependence of measured polarization on position, $\partial P / \partial x$, is determined by simulation. The simulation is benchmarked by looking at the relative and absolute rates of an actual position scan. When the simulation agrees with the actual study, we have improved confidence that our simulation is correct and can accurately determine the analyzing power. Figure 4.9 shows the relative rates from simulated position scans for the $x$ and $y$ directions, while Figure 4.10 shows the simulated polarization sensitivity from position. These scans were performed out to \pm 2.5 $\mathrm{mm}$ in both directions.

Figure 4.10 shows interesting non-linear behavior in polarization during the $x$ position scan. Similar behavior was also seen for the $y$ sensitivity. Since data was only taken within $\pm 0.5 \mathrm{~mm}$ from target center, making this non-lienar behavior irrelevant. It was determined that the optimal $\partial P / \partial x$ value is obtained from including only points for $\pm 1.5 \mathrm{~mm}$. 


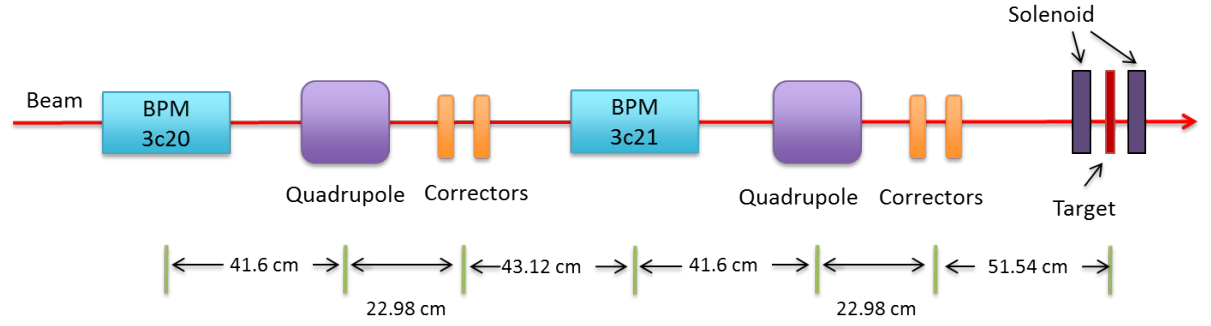

Figure 4.11: Diagram of optical elements immediately upstream of the Møller target.

The projection from the BPMs to the Møller target is a non-trivial calculation, as is the uncertainty of this projection, $\Delta x_{\text {calc }}$. Two BPMs lay immediately upstream of the Møller target. Information on beam position from these are used to project the actual beam position on target. Figure 4.11 shows the optical elements in this region consist of two quadruopoles and several beam corrector magnets. In particular, the target is placed inside of the superconducting solenoid; the coils are split, on either side of the iron foil.

A system of transport matrices $[98,99]$ is used to project the beam to the target. The simplest projection would be ignoring all optical elements, including the field as the beam enters the solenoid. The best projection would include the effects of these optical elements, and in particular the solenoid entrance field. ${ }^{3}$ Figure 4.12 shows the best multi-step transport algorithm.

The uncertainty in projecting from these BPMs to the target was calculated by seeing how different the beam position on target was between the simple method ( $\left.x_{\text {simple }}\right)$ and the best method $\left(x_{\text {best }}\right)$ :

$$
\Delta x=x_{\text {best }}-x_{\text {simple }} .
$$

The overall uncertainty contribution in projecting from the BPMs to the Møller target projection is given by

$$
\Delta P_{x-c a l c}=(\Delta x)(\partial P / \partial x) .
$$

Lastly, the uncertainty in the physical BPM position must be determined. The absolute BPM position accuracy was estimated to be good to $0.1 \mathrm{~mm}$. To be conservative, this estimate was doubled to $0.2 \mathrm{~mm}$, and we varied the position of the two BPMs individually to see the position difference at the target. The uncertainty of BPM position is then

$$
\Delta P_{\text {instr }}=\left(\Delta x_{\text {absolute }}\right)(\partial P / \partial x) .
$$

\footnotetext{
${ }^{3}$ Introducing this half-solenoid formalism provided substantial improvements to the position scan.
} 


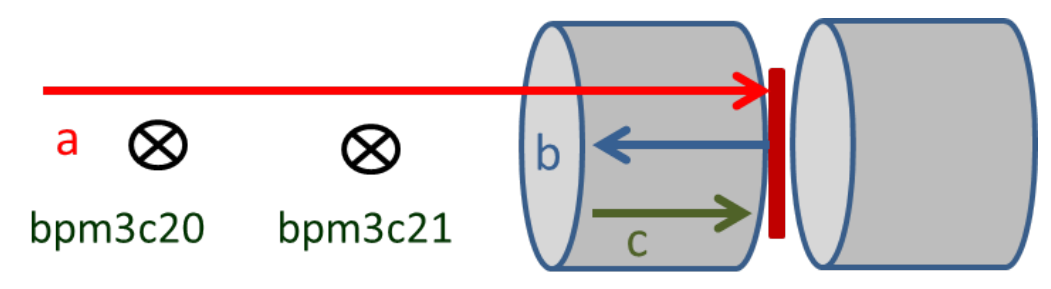

Figure 4.12: The procedure to calculate positions on the Møller target. (a) The BPM positions at $3 \mathrm{c} 20$ and $3 \mathrm{c} 21$ are transported to the target position using a transport formalism accounting for magneto-optical elements in the beam line but without using any solenoid magnetic field. (b) The beam is then drifted, with no magnetic field, back upstream to the solenoid entrance. (c) Finally, the beam is transported to the target using a transport formalism that only accounts for the entrance field (a half solenoid transport).

\begin{tabular}{l|lllllc} 
& $\partial P / \partial x$ & $\Delta x_{\text {best }- \text { simple }}$ & $\Delta P_{\text {calc. }}(\%)$ & $\Delta x_{\text {instr. }}$ & $\Delta P_{\text {inst. }}(\%)$ & $\sqrt{\Delta P_{\text {calc }}^{2}+\Delta P_{\text {instr }}^{2}}(\%$ \\
\hline$x$ position & 0.4461 & $0.280 \mathrm{~mm}$ & 0.14 & $0.08 \mathrm{~mm}$ & 0.036 & 0.14 \\
$y$ position & 0.5007 & $0.566 \mathrm{~mm}$ & 0.283 & $0.07 \mathrm{~mm}$ & 0.035 & 0.28 \\
$x$ angle & 0.3191 & $0.02 \mathrm{mrad}$ & 0.0064 & $0.1924 \mathrm{mrad}$ & 0.061 & 0.06 \\
$y$ angle & 0.1135 & $0.31 \mathrm{mrad}$ & 0.035 & $0.1924 \mathrm{mrad}$ & 0.022 & 0.04
\end{tabular}

Table 4.3: Summary of systematic uncertainty calculations from beam position/angle on target. The final column gives the combined systematic uncertainty to $A_{Z Z}$ in percent.

To determine the overall uncertainty of target position and angle, the quadrature sum was taken of $\Delta P_{\text {calc }}$ and $\Delta P_{\text {instrumental }}$. Table 4.3 summarizes the individual calculations for $x, y$ target position and angle.

\subsubsection{Møller Run 1 optics effect}

During Run 1 the large quadrupole (Q3) experienced a time-dependent turn-to-turn short, resulting in a varying analyzing power. This effect varied day-to-day and even during measurements; that is, some days the field appeared stable while other days it decayed rapidly during that day's measurement. It appears that the quadrupole was impaired throughout all of Run 1 and never performed optimally. Therefore the task was to determine each day's analyzing power and how stable it was.

Prior to the Run 1 position scan in May 2011, a pair of magnetic Hall probes were installed on the beam line inside of the large quadrupole, one on beam-left side and one on the beam-right side. The probe outputs were connected to a closed-circuit video system and read remotely in the counting house. The probe outputs were recorded every few minutes and at the start of every run. A plot of the beam right decay was seen in Figure 4.4. Whereas the position scan was a lengthy systematic procedure, taking over 6 hours to complete, most production data were taken over the span of 1-2 

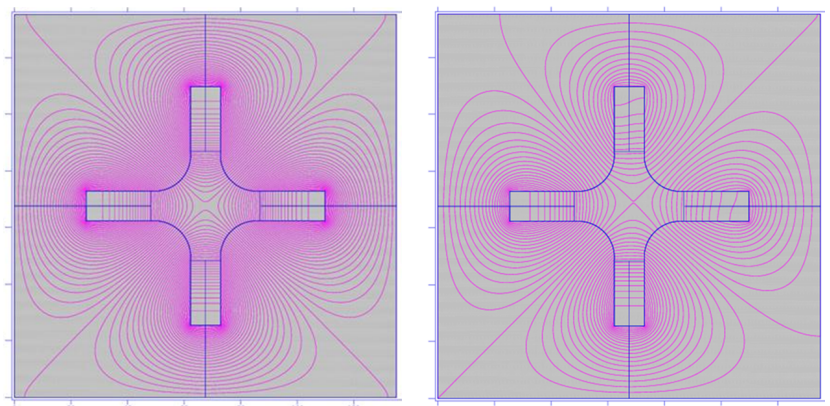

Figure 4.13: Magnetic field maps generated by POISSON for a fully functional quadrupole magnet (left) and a quadrupole magnet with a weak quadrant (right).

hours, where the decay is potentially largest.

A two-dimensional magnetostatic model of the quadrupole field was developed with POISSON [100] (Figure 4.13). Various models were developed, with each having a different field strength in the one bad quadrant. A quadrant's field strength is a function of either the number of coil turns, or the effective current running through the coil. In POISSON, the effective current was reduced in each model, producing the overall effect.

These models were then implemented into the Møller Monte Carlo, using a routine where the scattered electrons were iteratively swum through the magnetic field. This method was benchmarked in the Run 2 simulation with a fully functional quadrupole model. This routine produced identical results to the nominal transport algorithm.

The position scan study was crucial in benchmarking the simulation. Aside from providing a critical test of position dependence, it was over 6 hours long. During this period multiple tune plots were made, so the decay in field could be matched with a decay in tune. Two data points were taken at target center $(x=0, y=0)$, separated in time by six hours. The changes in rates were also monitored as an important field diagnostic. Originally the relative rate decay from the initial point was used, but yielded inconsistent results with simulations. It became apparent that matching the derivative was a stronger condition, yielding consistent simulation results. Figure 4.14 shows the relative rates as a function of quadrupole field from the Hall probe (measured about halfway between the beam right coils). A detailed calculation showed the relative rate decay derivative is best matched at $92 \%$ measured field on this particular day. This is equivalent to the quadrupole having an effective current $84 \%$ of nominal.

Once the simulation was benchmarked using the field and rate decay metrics, each Møller measurement in Run 1 was individually simulated. Conditions, such as measured beam positions on target, were used as simulation inputs. This is in contrast to Run 2 , 


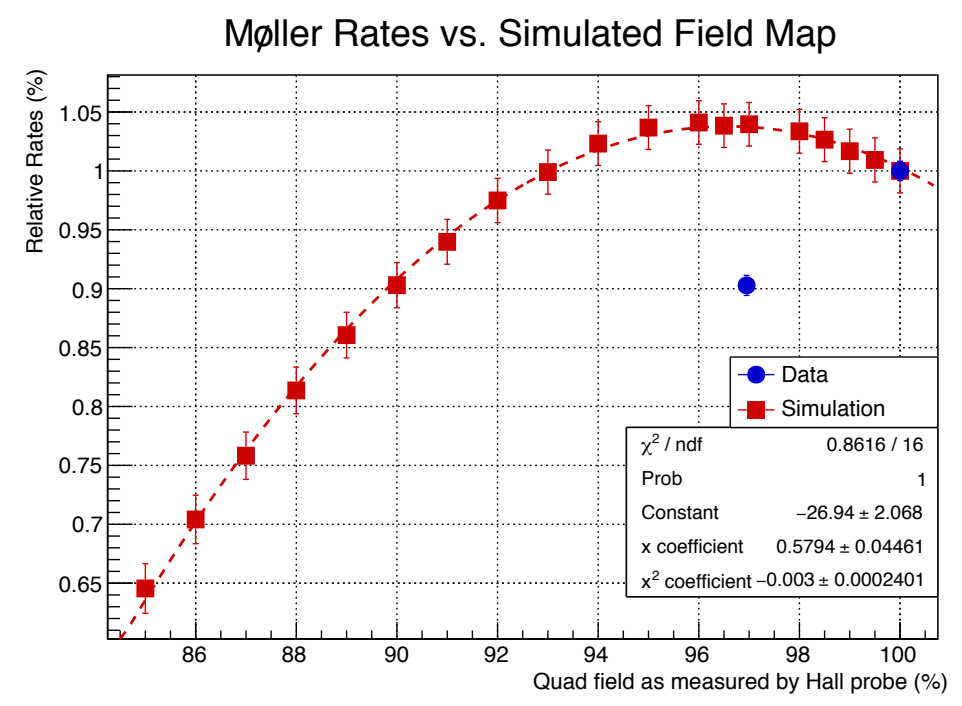

Figure 4.14: Relative scattering rate in the Møller during the position scan. Data are blue circles, taken with beam directed at the target center separated by six hours. Simulation is red squares. The fit was used to determine the scattering rate derivative, and was compared to the change seen in data.

where the measured position-dependence was applied as a correction (it was not explicitly simulated). Each day's measurement was simulated using 20 different POISSON field maps, from nominal coil current to an effective coil current $60 \%$ of nominal, in $2 \%$ effective coil current increments. A matrix of field map correlation plots was generated for each day, and was used to determine that day's starting field. Since the rate decay is tightly correlated with field decay, the relative rate drop over the span of the day's measurement was used to determine how quickly the field was actually decaying, and the field at the conclusion of that day's measurement. The average analyzing power of this range was used and the standard deviation of all the possible analyzing powers taken as the uncertainty for that day.

Simulations suggested that the system analyzing power could change $1.3 \%$ from nominal quadrupole coil current to $70 \%$ of nominal. Half of this value $(0.65 \%)$ was used as a systematic scale or normalization uncertainty for potential mis-calibration of the simulation model. This value of $70 \%$ was chosen because only two measurements exhibited problems this large, effectively bounding the effect.

The systematic uncertainty for Run 1 is $1.10 \%$ overall. Although not meeting the original $Q_{\text {weak }}$ goal of $1 \%$, this is remarkable considering the potential impact of the quadrupole short. 


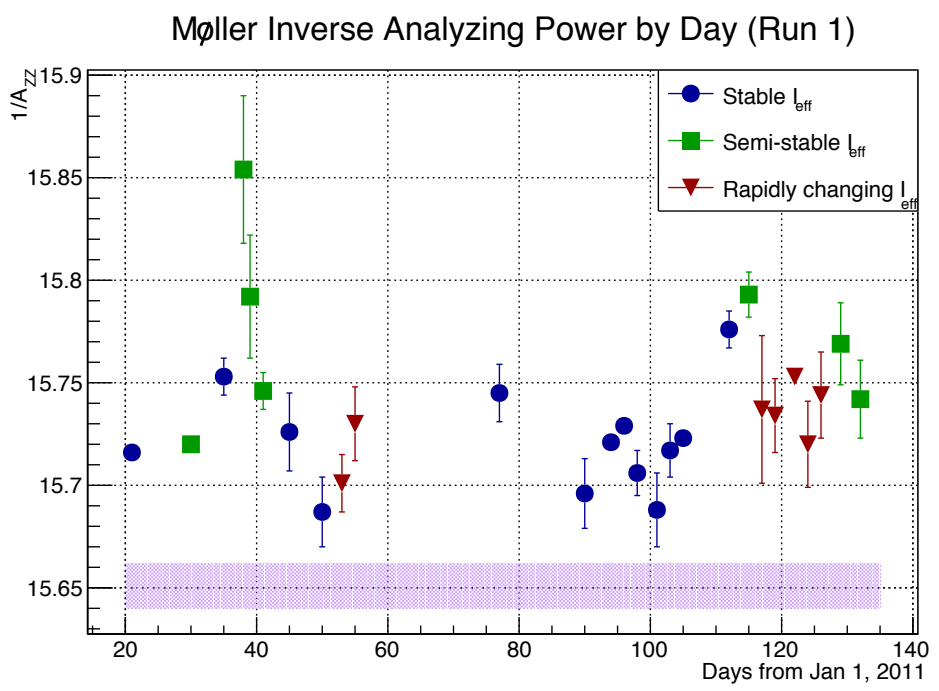

Figure 4.15: Plot of the simulated inverse analyzing power versus day during Run 1. Blue circles are measurements when the large quadrupole appeared stable, green squares are when the field appears to change several percent, and red inverted triangles are when the field was rapidly changing. These designations are determined by the observed rate decay over the span of that day's measurement and the correlation-tune plots. The purple band is the 1- $\sigma$ common systematic uncertainty from Monte Carlo statistics.

\section{Final table of uncertainties}

The final base table of Møller systematic uncertainties is given in Table 4.4. Extra uncertainties are assigned to Run 1, which are addressed in Table 4.5.

A single 5 minute long Møller measurement typically provided $0.5 \%$ statistical precision. With 3 such runs per measurement, the full statistical contribution for each measurement was about $0.3 \%$, often less. Averaged over longer run periods the statistical uncertainty becomes negligible.

Systematic uncertainties can be split into two kinds: those that are point-to-point, and those of scale (or normalization). Point-to-point uncertainties vary in each measurement. For example, beam position on target changes for each measurement. Normalization uncertainties affect all measurements equally; their true value may shift up or down within their uncertainty band. Some measurements have components that exhibit both types of behavior, notably the solenoid position and high-current extrapolation uncertainties.

The solenoid position uncertainty is divided evenly between point-to-point and normalization types. When the solenoid is cooled, the coils' transverse position shifts, as discussed in Section 4.2.3. We allow for some lack of reproducibility in the cooldown motion by dividing the overall uncertainty evenly between scale and point-to-point 
types. In this way, it exhibits both time-dependent and time independent behavior.

The high-current extrapolation uncertainty is also divided between the two systematic uncertainty sub-types. Some concern exists that the polarization is measured at $2 \mu A$ with the Møller polarimeter, while $Q_{\text {weak }}$ data taking is typically done at 180 $\mu A$. The concern is that there might be some current-dependence in the produced electron's polarization. There is currently no widely accepted mechanism for such a dependence, although a correlation between beam polarization with quantum efficiency has been observed. Quantum efficiency is a function of polarized source laser power, so it is possible that the higher laser powers during production running could result in a different effective quantum efficiency than that during Møller measurements. However, quantum efficiency was monitored daily during $Q_{\text {weak }}$, and the correlation with quantum efficiency decreased with the laser spot size increase in Run 2 (discussed in Section 3.2.1). Moreover, no direct observational evidence of a current-dependence up to $60 \mu \mathrm{A}$ has been observed in previous studies using a Møller raster, magnetic kicker system [110, 113], and a special beat frequency system [114].

With the installation of the Compton polarimeter, a cross-calibration shows no current dependence at the $0.70 \%$ level. This is discussed in Appendix A. A $0.50 \%$ uncertainty is included for this effect to be conservative. This is split between pointto-point and normalization types as this effect, if it exists, may vary day-to-day.

\subsection{Data and results}

\subsubsection{Leakage corrections}

After most Møller measurements, a few minutes were dedicated to measuring the electron beam leakage from the other halls. Typically this leakage was small (on the order of 10 's of $\mathrm{Hz}$ ), however the overall contribution could be on the order of $0.1 \%$, sometimes larger. This is because the produced electron beams in Halls A and B are of opposite polarity due to the use of the polarizing cube in the injector (see Section 3.2.1). The measured polarization and the true polarization, $P_{\text {meas }}$ and $P_{\text {true }}$, are related by

$$
P_{\text {meas }}=P_{\text {true }} f_{C}-P_{\text {true }} f_{A+B}
$$

where $f_{i}$ is the rate fraction from Hall $i$. The rate fractions sum to unity:

$$
f_{A+B}+f_{C}=1 .
$$

Therefore the measured polarization is given by

$$
P_{\text {meas }}=P_{\text {true }}\left(1-2 f_{A+B}\right) \text {, }
$$




\begin{tabular}{|l|c|c|c|c|}
\hline Source & Uncertainty & $\mathrm{dA} / \mathrm{A}(\%)$ & Scale & Point-to-point \\
\hline Beam position X & $0.2 \mathrm{~mm}$ & 0.14 & - & 0.14 \\
\hline Beam position Y & $0.2 \mathrm{~mm}$ & 0.28 & - & 0.28 \\
\hline Beam angle X & $0.5 \mathrm{mrad}$ & 0.10 & - & 0.10 \\
\hline Beam angle Y & $0.5 \mathrm{mrad}$ & 0.10 & - & 0.10 \\
\hline Q1 current & $2 \%$ & 0.07 & - & 0.07 \\
\hline Q3 current & $2 \%$ & 0.05 & - & 0.05 \\
\hline Q3 position & $1 \mathrm{~mm}$ & 0.10 & 0.01 & - \\
\hline Multiple scattering & $10 \%$ & 0.01 & 0.01 & - \\
\hline Levchuk effect & $10 \%$ & 0.33 & 0.33 & - \\
\hline Fixed collimator positions & $0.5 \mathrm{~mm}$ & 0.03 & 0.03 & - \\
\hline Target temperature & $100 \%$ & 0.14 & 0.14 & - \\
\hline B-field direction & $2 \mathrm{degrees}$ & 0.14 & 0.14 & - \\
\hline B-field strength & $5 \%$ & 0.03 & 0.03 & - \\
\hline Spin polarization in Fe & - & 0.25 & 0.25 & - \\
\hline Electronic D.T. & $100 \%$ & 0.045 & 0.045 & - \\
\hline Solenoid position (x,y) & $0.5 \mathrm{~mm}$ & 0.23 & $0.23 / \sqrt{2}$ & $0.23 / \sqrt{2}$ \\
\hline Solenoid focusing & $100 \%$ & 0.21 & 0.21 & - \\
\hline High-current extrapolation & - & 0.50 & $0.50 / \sqrt{2}$ & $0.50 / \sqrt{2}$ \\
\hline Monte Carlo statistics & - & 0.14 & - & - \\
\hline Total & & $\mathbf{0 . 8 4}$ & $\mathbf{0 . 6 4}$ & $\mathbf{0 . 5 3}$ \\
\hline
\end{tabular}

Table 4.4: Final Møller systematic uncertainty list for the $Q_{\text {weak }}$ Run 2 period. Note the conservative inclusion of high-current extrapolation uncertainty applied to the $Q_{\text {weak }}$ results. The last two columns separate the systematic uncertainty in two parts: scale denotes systematics common to all data, while point-to-point are time-dependent contributions which vary between measurements. 


\begin{tabular}{|l|c|c|c|c|}
\hline Source & Uncertainty & $\mathrm{dA} / \mathrm{A}(\%)$ & Scale & Point-to-point \\
\hline Beam position X & $0.2 \mathrm{~mm}$ & 0.17 & - & 0.17 \\
\hline Beam position Y & $0.2 \mathrm{~mm}$ & 0.28 & - & 0.28 \\
\hline Beam angle X & $0.5 \mathrm{mrad}$ & 0.10 & - & 0.10 \\
\hline Beam angle Y & $0.5 \mathrm{mrad}$ & 0.10 & - & 0.10 \\
\hline Q1 current & $2 \%$ & 0.07 & - & 0.07 \\
\hline Q3 current & $2 \%$ & 0.05 & - & 0.05 \\
\hline Q3 position & $1 \mathrm{~mm}$ & 0.10 & 0.01 & - \\
\hline Multiple scattering & $10 \%$ & 0.01 & 0.01 & - \\
\hline Levchuk effect & $10 \%$ & 0.33 & 0.33 & - \\
\hline Fixed collimator positions & $0.5 \mathrm{~mm}$ & 0.03 & 0.03 & - \\
\hline Target temperature & $100 \%$ & 0.14 & 0.14 & - \\
\hline B-field direction & 2 degrees & 0.14 & 0.14 & - \\
\hline B-field strength & $5 \%$ & 0.03 & 0.03 & - \\
\hline Spin polarization in Fe & - & 0.25 & 0.25 & - \\
\hline Electronic D.T. & $100 \%$ & 0.045 & 0.045 & - \\
\hline Solenoid position $(x, y)$ & $0.5 \mathrm{~mm}$ & 0.23 & $0.23 / \sqrt{2}$ & $0.23 / \sqrt{2}$ \\
\hline Solenoid focusing & $100 \%$ & 0.21 & 0.21 & - \\
\hline High-current extrapolation & - & 0.50 & $0.50 / \sqrt{2}$ & $0.50 / \sqrt{2}$ \\
\hline Analyzing power nominal value & $50 \%$ of change & 0.65 & 0.65 & - \\
\hline Analyzing power variation & $100 \%$ of deviation & $0.019-0.230$ & - & $0.019-0.230$ \\
\hline Monte Carlo statistics & $0.024 /$ polfac & 0.15 & - & 0.15 \\
\hline Total & & $\mathbf{1 . 0 7 - 1 . 0 9}$ & $\mathbf{0 . 9 2}$ & $\mathbf{0 . 5 5 - 0 . 5 9}$ \\
\hline
\end{tabular}

Table 4.5: Final Møller systematic uncertainty list for the $Q_{\text {weak }}$ Run 1 period. These optics uncertainties come in two parts: an overall scale/normalization uncertainty, and an additional uncertainty due to that day's rate of field decay. 
where the leakage rate fraction, $f_{A+B}$, was typically measured to be of the order of $0.1 \%$.

Leakage measurements were taken with the Hall $\mathrm{C}$ laser off, the slit in its nominal position (wide open), and the Møller detector coincidence rates were observed. In this configuration, the produced electrons seen were produced by either the Hall A or B lasers.

For Run 2, the leakage correction was small (on the order of $0.1 \%$ ), as both Halls A and B were running at low-current (in the nA range). However, during Run 1 the leakage contribution was occasionally quite large. This is due to lower Møller coincidence rates from the quadrupole problem coupled with Hall A running at high current $(\sim 120 \mu A)$. The combination of these effects meant the leakage contribution were up to $1.5 \%$ during a short period.

\subsubsection{Effect of the insertable half wave plate}

It was suggested that the primary slow helicity reversal method (inserting a half-wave plate before the Pockels Cell) might introduce subtle effects into the beam, and even alter the polarization. During Run 2, a second inactive half-wave plate was introduced ${ }^{4}$ to study this possibility. This half-wave plate had the fast axis along the polarization direction, leaving the incoming laser polarization unaltered ${ }^{5}$. In this short section, the effect of both half-wave plates and slow Wien reversal on polarization are also shown to be negligible.

To study the inactive half-wave plate, each day's polarization values were averaged for IHWP \#2 IN and OUT. Only measurements taken without IHWP \#1 were used at this point, otherwise its potential effect would be included as well. The ratio of IHWP \#2 IN/OUT should be unity if the half-wave plate is truly inactive. Figure 4.16 shows this the case. Once it was shown that the inactive half-wave plate had no effect on the polarization, a similar study was undertaken on the active half-wave plate (IHWP \#1). For this study the inactive half-wave plate was averaged over. Figure 4.17 shows agreement at the 1-sigma level, or better (for Wien flip left). If one includes the point-to-point systematic uncertainty, both Wien states agree within their respective uncertainties.

Lastly, the final polarization values for each Wien flip are given by day in Figure

\footnotetext{
${ }^{4}$ This insertable half-wave plate was colloquially known as the Carlini plate, in honor of $Q_{\text {weak }}$ spokesman Roger Carlini.

${ }^{5}$ Through studies with the inactive half-wave plate it was shown that inserting these optical elements shifts the beam bunches slightly off crest of the accelerator timing, but ultimately was demonstrated to have negligible effect on the elastic $\vec{e} p$ asymmetry.
} 


\section{Effect of IHWP 2 on Measured Polarization (Run 2)}

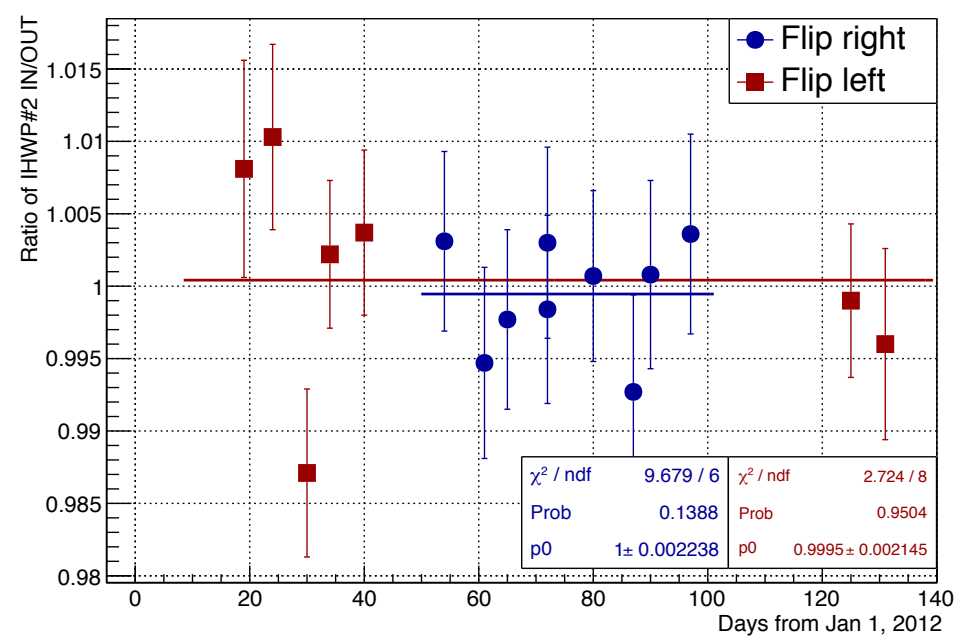

Figure 4.16: The ratio of the measured polarizations with the inactive half-wave plate inserted to those without versus time for each Wien flip. Only statistical uncertainties are shown. Note the excellent agreement between Wien flip right and flip left states from the $\chi_{d o f}^{2}$, indicating no IHWP \#2 effect on polarization, regardless of the Wien flip state. IHWP \#1 was out for all runs shown. Taken from [115].

Effect of IHWP 1 on Measured Polarization (Run 2)

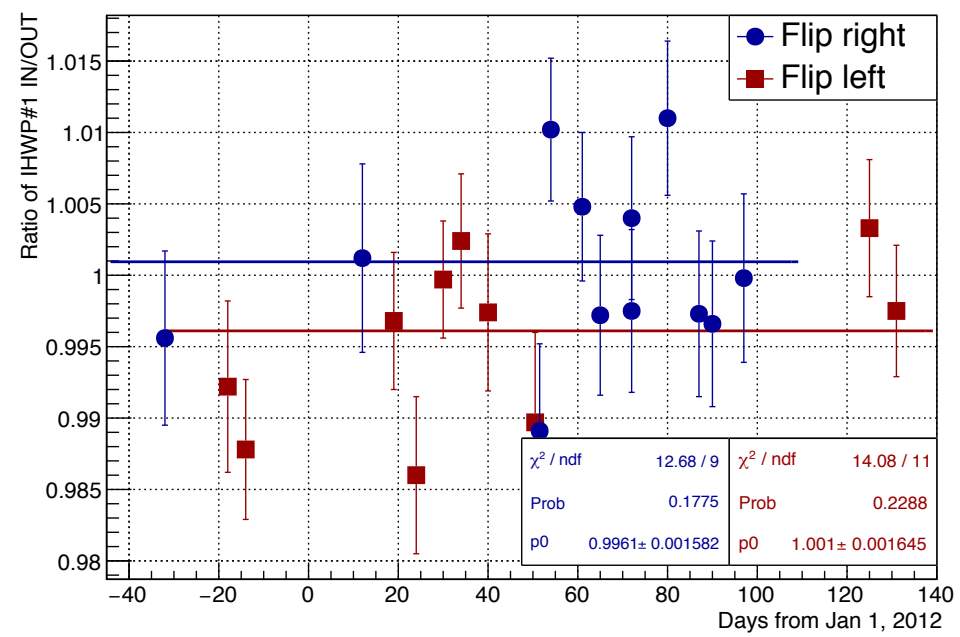

Figure 4.17: The ratio of the measured polarizations with the active half-wave plate inserted to those without IHWP \#1 In/Out versus time. Only statistical uncertainties are shown. Note the agreement between both Wien flips and the reasonable $\chi_{d o f}^{2}$. Note also there is a 2.5-sigma discrepancy for Wien flip right. Including the point-to-point systematic uncertainty eliminates this discrepancy (see Figure 4.18 for an explanation of this point-to-point uncertainty value). 


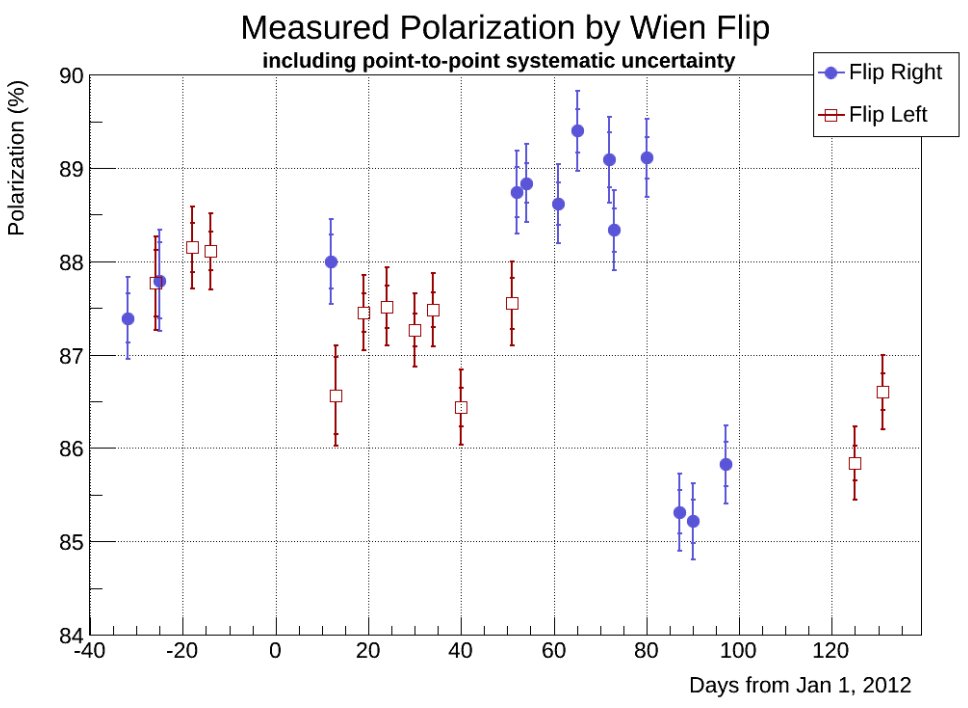

Figure 4.18: Polarization for each day, sorted by Wien flip in Run 2. No fits were included as there is a clear time dependence on polarization. Note a possible systematic difference between Wien states may exist. The outer uncertainty bars includes a $0.40 \%$ point-to-point systematic uncertainty. From Table 4.4, the typical measurement pointto-point uncertainty is $0.54 \%$, however since all Møller measurements were taken at low-current, the high-current extrapolation uncertainty is removed here.

4.18. Note an additional $0.40 \%$ point-to-point uncertainty was added. This uncertainty is due to point-to-point systematic uncertainties, with the high-current extrapolation uncertainty removed. Note there is clear time dependence of the polarization, which is not unexpected.

\subsection{Møller summary}

The Møller polarimeter consistently made sub-percent determinations of electron beam polarization throughout Run 2. The overall systematic uncertainty of the device in Run 2 was $0.85 \%$ and showed consistency with the Compton polarimeter as seen in Figure 4.19. The average difference between the two polarimeters was $\sim 0.7 \%$. A Møller and Compton cross calibration occured during Run 2, and is the subject of Appendix A. For Run 1, the Møller consistently made percent-level determinations of the electron beam polarization, with a final overall systematic uncertainty of $1.10 \%$ (see Figure 4.20 ). This was heavily dependent on modeling of the large quadrupole, which experienced an intermittent electrical short in one coil. This electrical short resulted in a time dependent magnetic field in one quadrant. 


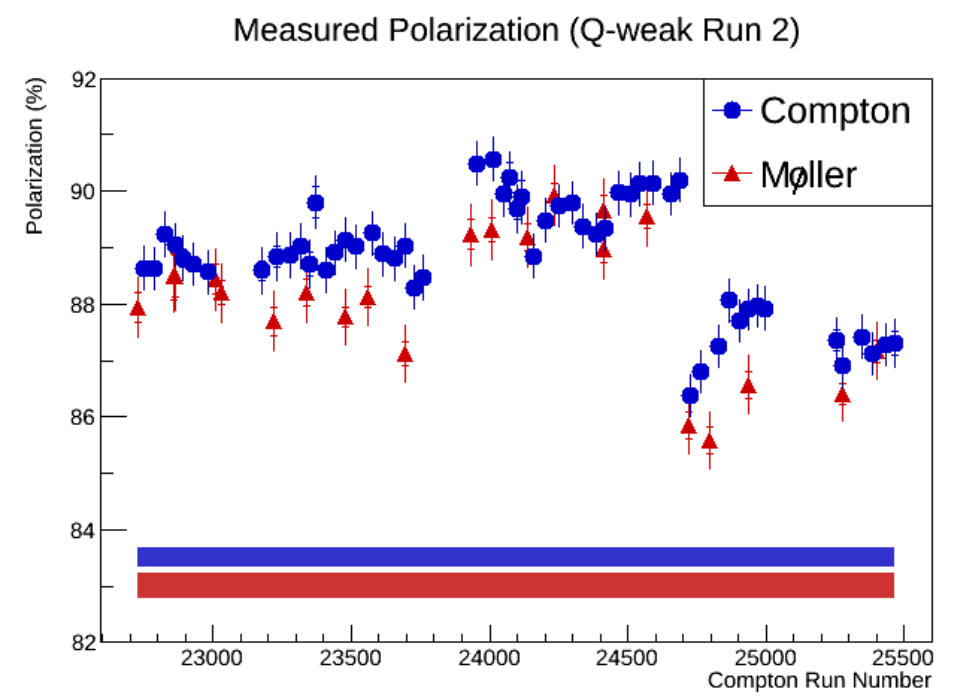

Figure 4.19: Final $Q_{\text {weak }}$ Run 2 polarimetry results for both Møller (red triangles) and Compton (blue circles). Note the good consistency between the two independent subpercent devices. The results here are plotted versus Compton run number; this spans over 6 months of data taking from November 2011 through May 2012 and encompasses thousands of hours of data. Each Compton point is an average of 30 hours of data.

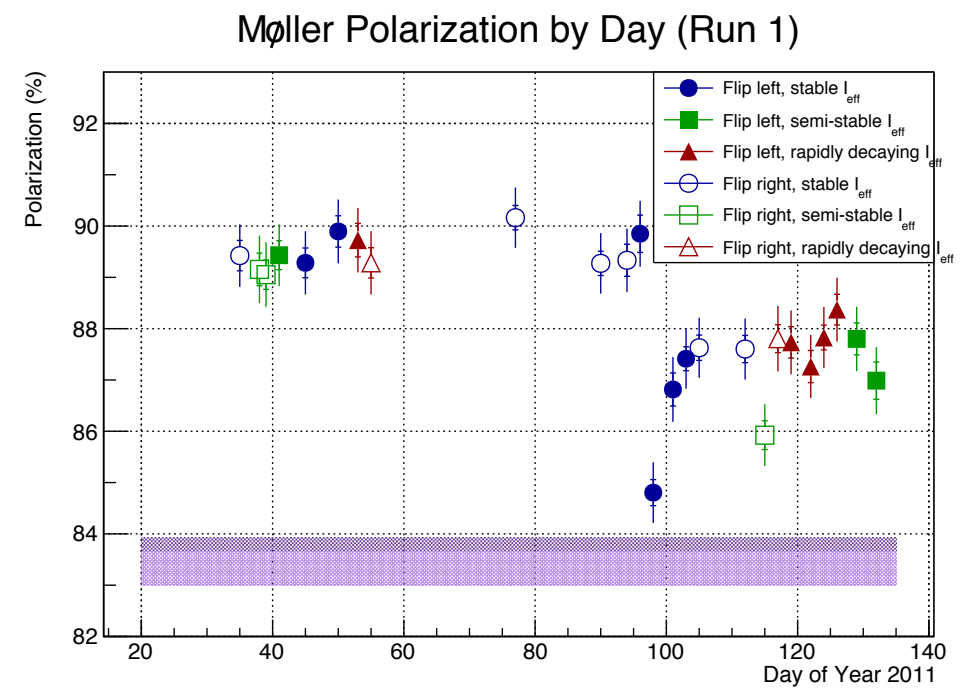

Figure 4.20: Final $Q_{\text {weak }}$ Run 1 polarization results. The Compton electron detector began taking data around Day 90, but is neglected here. Data points are separated into Wien flip left (closed markers) and flip right (open marker) states. Each day's quad stability was determined by analyzing the change in scattering rate to be stable (blue circles), semi-stable (green squares), or rapidly changing (red triangles). The lightpurple band gives the common 1- $\sigma$ systematic uncertainty, while combined with the darker purple band is the total systematic uncertainty, including a $0.65 \%$ uncertainty for the quadrupole model. 


\section{Chapter 5}

\section{The Aluminum Window Contribution}

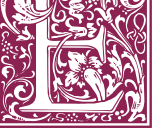

XPERIMENTS WHICH INTEGRATE their signals cannot reject background events through pre-event selection since all backgrounds are intrinsically part of the signal. Instead, backgrounds must be anticipated and suppressed through design, and precisely measured during experimental running and subtracted during analysis. The largest experimental correction in $Q_{\text {weak }}$ comes from the target's aluminum windows, which generates approximately $30 \%$ of measured physics asymmetry. Previously published results from Run 0 determined this correction to only $15 \%$ relative precision [48], and are inadequate for the final $Q_{\text {weak }}$ results, which require knowledge of this correction to a precision of only a few percent.

Backgrounds typically have two components: the rate fraction, or dilution, and the signal size (its asymmetry). This chapter presents an in-depth analysis of the target window dilution, while the Chapter 6 discusses the extracted parity-violating asymmetry of the aluminum alloy. Although critical for $Q_{\text {weak }}$, these particular results will ultimately provide the first measurement of the parity-violating asymmetry of aluminum, which has important ramifications in its own right.

\subsection{Aluminum overview}

Each background contribution has two pieces: the rate-fraction that background process contributes to the overall yield (the dilution, $f$ ), and the overall asymmetry size it contributes $(A)$. For $Q_{\text {weak }}$, a measurement of the parity-violating asymmetry in hydrogen, the measured asymmetry, $A_{\text {meas }}$, is related to the true physics asymmetry, $A_{\text {phys }}$, by

$$
A_{\text {meas }}=P\left[\left(1-\sum_{i} f_{b k g d}^{i}\right) A_{\text {phys }}+\sum_{i} f_{b k g d}^{i} A_{b k g d}^{i}\right],
$$


where $i$ runs over the possible background sources (aluminum target walls, backgrounds from beamline scattering, neutral particle backgrounds, inelastic contributions), $P$ is the electron beam polarization, and radiative effects have been neglected. This chapter focuses on the aluminum target wall dilution, while Chapter 6 focuses on determination of the parity-violating asymmetry of the target's aluminum alloy. $Q_{\text {weak }}$ 's treatment of the target asymmetry is unique; while previous parity-violating experiment's simulated the asymmetry of their target walls, $Q_{\text {weak }}$ directly measured the parity-violating asymmetry of elastic electron-aluminum scattering.

Figure 5.1 shows the predicted elastic cross-section and asymmetry for $e+p$ and $e+A l$ scattering. Note that the elastic cross-section drops over an order-of-magnitude over the $Q_{\text {weak }}$ acceptance, suggesting a large contribution in rate from the upstream window (smaller angle). In addition, the parity-violating asymmetry for aluminum, $A_{P V}(A l)$, is over an order-of-magnitude larger than that for elastic $e+p$ scattering; although the neutral-weak coupling of the proton is suppressed, it is unity for the neutron, and aluminum is neutron rich. Here, the parity-violating asymmetry for elastic electron-aluminum scattering is given by [46]:

$$
\begin{aligned}
A_{P V}\left({ }^{27} A l\right) & =-\frac{G_{F} Q^{2}}{4 \pi \alpha \sqrt{2}}\left[Q_{W}^{p}+\left(\frac{N}{Z}\right) Q_{W}^{n}\right] \\
& \approx 2200 \mathrm{ppb} \text { at } Q^{2}=0.025 \mathrm{GeV}^{2} .
\end{aligned}
$$

In all, if we take the Standard Model value for $A_{P V}(H) \approx 200 \mathrm{ppb}$, this suggests the total contribution from the target's aluminum windows is $\approx 30 \%$ :

$$
\begin{aligned}
f \cdot A_{P V} & \approx 3 \% \cdot 2200 \mathrm{ppb} \\
& =66 \mathrm{ppb} .
\end{aligned}
$$

To reduce sensitivity to the upstream window, $Q_{\text {weak }}$ designed the upstream window profile to be partially off the main detector. When the target is filled with $\mathrm{LH}_{2}$, the additional radiative losses then pull the scattering profile onto the bar, as seen in Figure 5.2. This is in contrast with the downstream window profile which sits completely on the detector; any radiative losses due to the addition of hydrogen cause small shifts in $Q^{2}$ and therefore the asymmetry size, but the acceptance remains virtually unchanged. These higher-order corrections are crucial in calculating the window dilution, and are the subject of Section 5.4.

Currently, these higher-order corrections are the dominant contribution in the Run 0 dilution (7.5\%). The effect of the dilution's uncertainty on the uncertainty of $A_{e p}$ can 

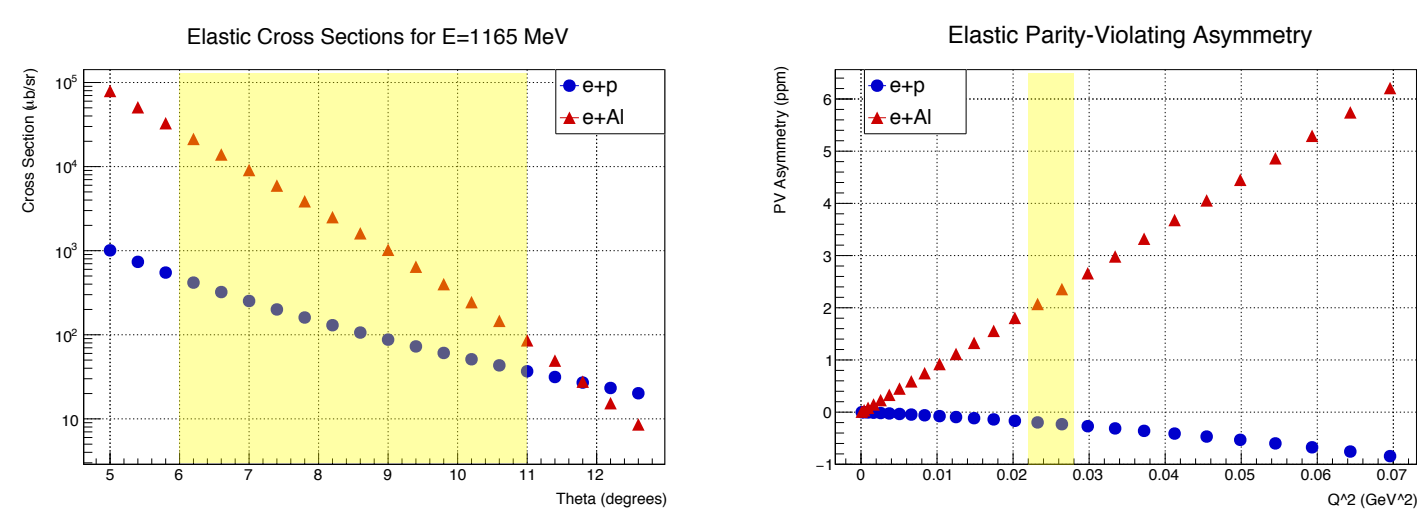

Figure 5.1: (Left) Elastic cross-section and (Right) parity-violating asymmetry for $e+p$ (blue circles) and $e+A l$ scattering (red triangles). The yellow-box shows the $Q_{\text {weak }}$ experimental acceptance. Note the sharp decline in the aluminum cross-section, suggesting increased sensitivity to the upstream window.

US Aluminum Target Profile

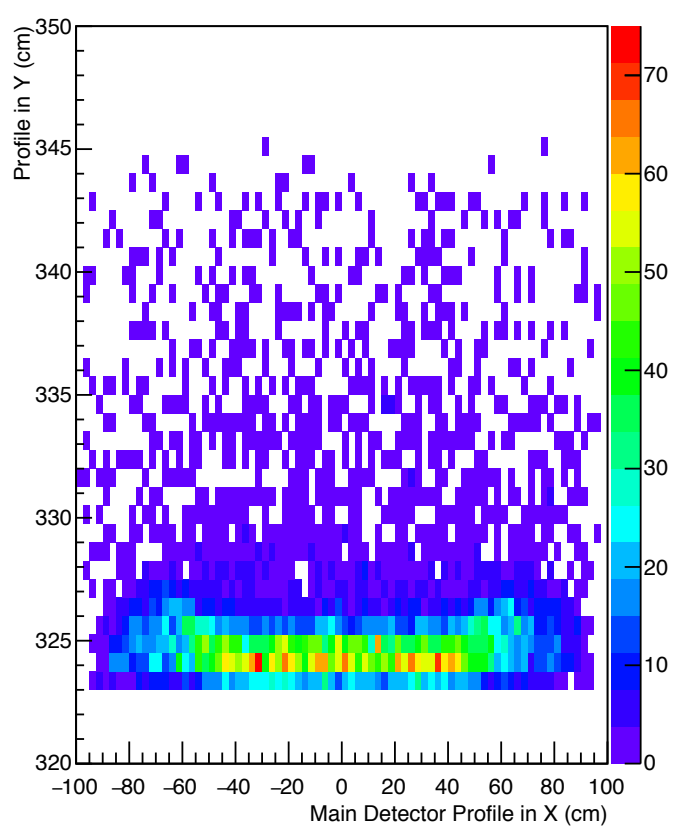

US Aluminum Target Profile (including hydrogen)

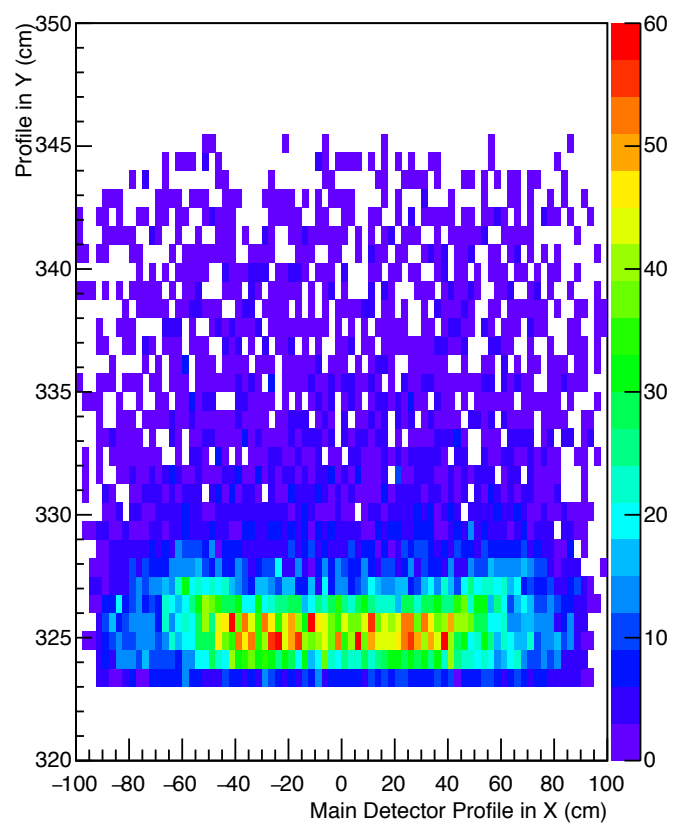

Figure 5.2: Simulated scattering profile from the target upstream aluminum target window in (left) an empty target and (right) a target full of LH2. Note how the addition of hydrogen shifts the profile upwards onto the detector through radiative effects and energy losses. 
be found by taking derivatives of (5.1):

$$
\left(\frac{d A_{e p}}{A_{e p}}\right) \cong d f \frac{A_{A l}}{A_{e p}}=\left(\frac{d f}{f}\right) \cdot\left(\frac{f A_{A l}}{A_{e p}}\right) .
$$

This implies that a $2 \%$ relative determination of the dilution $(f)$ would give us a $0.66 \%$ contribution to the desired $Q_{\text {weak }}$ physics asymmetry $A_{e p}$, which would be adequate.

\subsection{Dilution method}

The dilution is simply the rate fraction of events scattered from the target cell walls compared to the total scattering rate from a full target, and is determined to first-order by taking the ratio of two experimentally measured quantities:

$$
f_{\text {raw }}=\frac{Y_{\text {empty }}}{Y_{\text {full }}},
$$

where $Y_{\text {empty }}\left(Y_{\text {full }}\right)$ is the current-normalized rate, or yield, of the empty (full) target.

There are two ways to determine the empty cell rate: one can directly measure the empty cell, or one can fill the target with cold gaseous hydrogen at various pressures and extrapolate to zero-gas pressure. Measuring the empty cell is the simplest approach and the most precise, while the $H_{2}$ gas running provides thermal protection for the windows but includes larger systematic uncertainties from the extrapolation. ${ }^{1}$

Measurements with gaseous hydrogen were done in both Run 1 and Run 2, but problems with maintaining constant gas pressure were present in Run 2, rendering that particular study fruitless. The results of the Run 1 gaseous measurements and their interpretation were discussed in [42], and are not covered here.

Ideally, both empty and full target measurements would be taken at identical currents to minimize any beam current monitor (BCM) uncertainties. In practice, the full rate measurements were taken at about $100 \mathrm{nA}$ while the empty target runs were taken at $1 \mu \mathrm{A}$. When full of $\mathrm{LH}_{2}$, the scattering rate was large, and limited data-taking to low-currents. It was hoped that at higher currents the empty target rates would more closely match those during full target running, enabling similar accidental and dead time correction sizes. ${ }^{2}$

\footnotetext{
${ }^{1}$ The target walls are thin, and the liquid hydrogen provides thermal cooling which prevents damage. One could argue for using extremely low gaseous $H_{2}$ pressures to minimize extrapolation uncertainties, but then the dissipative benefits of using gas likewise vanish.

${ }^{2}$ It is clear that placing high $\sim \mu A$ currents on an empty target is distressing due to the aforementioned possibility of thermal damage, but the arguments against taking long runs at low-current on an empty target are less clear. Since $Q_{\text {weak }}$ had no back-up hydrogen target, any target damage would be catastrophic. It was believed to be safer to run at a moderately high current for a short time than at low-current for an hour, as this minimized potential operator and beam delivery errors.
} 
Taking measurements at multiple beam currents increases sensitivities to BCM calibration and any associated non-linearity. To account for these, a solid reference "dummy" target was planned as an alternate normalization method. Since yields are independent of current, the ratio of reference target yields at both high- and low- currents should be unity, and any systematic BCM problems will divide out to leading order. This technique was crucial, making several rate-dependent problems immediately evident by the non-unity ratio. ${ }^{3}$ The reference target analysis is covered in Section 5.2 .2 .

Surprisingly, the most crucial and difficult decision was deciding exactly how to calculate the rate. Three different ways of calculating the raw rates were developed, and although sharing similarities, are distinct. The three techniques are

- Scaler-based analysis

- Event-mode analysis

- Hybrid analysis.

The hybrid analysis uses pieces of both the scaler and event-mode analysis to assemble expedient and unambiguous results. As this author was heavily involved in developing these analyses, a full description of the scaler and event-mode techniques are found in Appendix B, including the final results and explanation of why they were ultimately not used. ${ }^{4}$

\subsubsection{Measurement conditions}

All aluminum dilution data were acquired under specific conditions. They were part of the low-current tracking periods, and used currents between $100 \mathrm{nA}-1 \mu \mathrm{A}$. Data were triggered using a combination of the trigger scintillators and the $10 \mathrm{~Hz}$ clock, the latter being known as the fake MPS. The trigger scintillators were typically positioned in octants 1 and 5 for each measurement, although a data subset was taken with them rotated into the other octants. The clock was used as the read-out for the scaler banks, and these were only read out when neither the DAQ nor trigger scintillator trigger was busy. The number of missed scaler read-outs were recorded to provide a deadtime correction. Diagrams of the DAQ and relevant trigger scintillator detector electronics can be seen in Figures 5.3.

\footnotetext{
${ }^{3}$ The reference target also reduces other time-dependent systematic effects such as gain drifts. However, these were found to be negligible in practice.

${ }^{4}$ The basic problem becomes apparent in Section 5.2.1: the main detector has a long analog pulse
} 


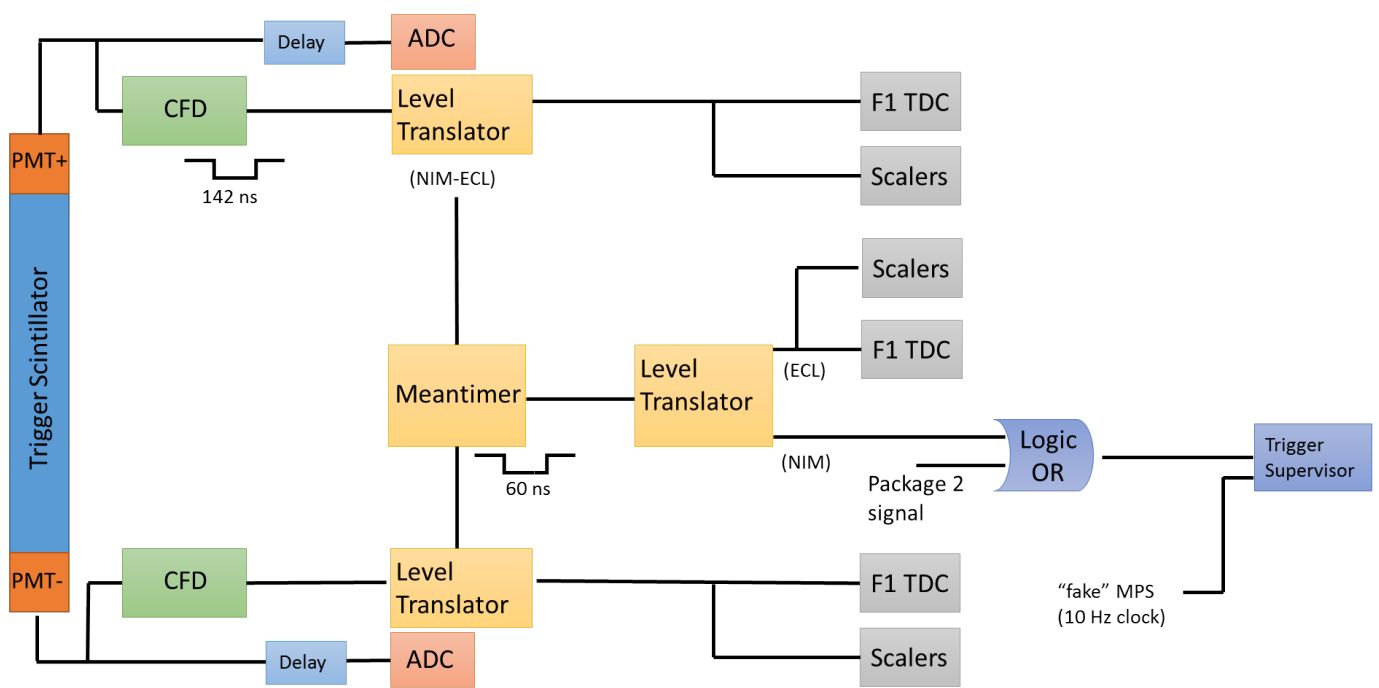

Figure 5.3: Electronics diagram for the low-current tracking mode. The trigger scintillators and a $10 \mathrm{~Hz}$ clock were used as triggers.

One key feature of the dilution analysis was the use of F1 time-to-digital converters (F1TDCs). These were implemented primarily for track reconstruction with the HDCs and VDCs, but were also implemented in the trigger scintillator and main detector front-end electronics. The F1TDCs are high resolution (10 ps) multi-hit pipeline TDCs $[116,117,118]$. Each time window opens when a trigger scintillator trigger occurs (called an event), and is open for $2 \mu \mathrm{s}$. Within this window a maximum of 7 hits are recorded in each TDC. The ability to distinguish multi-hit structures was a crucial part of the analysis, allowing investigations into our detector double pulse resolutions. Figure 5.4 gives an example hit selection process for a given trigger window. Appendix $\mathrm{B}$ discusses the implementation of F1TDCs and combining the results across multiple detectors to eliminate accidental backgrounds.

The calculated yields $(\mathrm{kHz} / \mathrm{nA}), Y$, can be determined with knowledge of only the four primary quantities

$$
Y=\frac{R-R_{a c c}}{\left(L T_{\text {electronic }}\right) \cdot\langle I\rangle},
$$

where $Y$ is the calculated yield $(\mathrm{MHz} / \mu A), R$ is the raw measured scattering rate, $R_{a c c}$ is the accidentals rate, $L T_{\text {electronic }}$ is the main detector electronic livetime, and $\langle I\rangle$ is the average beam current during the period.

shape. But a more subtle problem exists with the trigger, which apparently misses events at very high rates. This is explained in detail in Appendix B. 


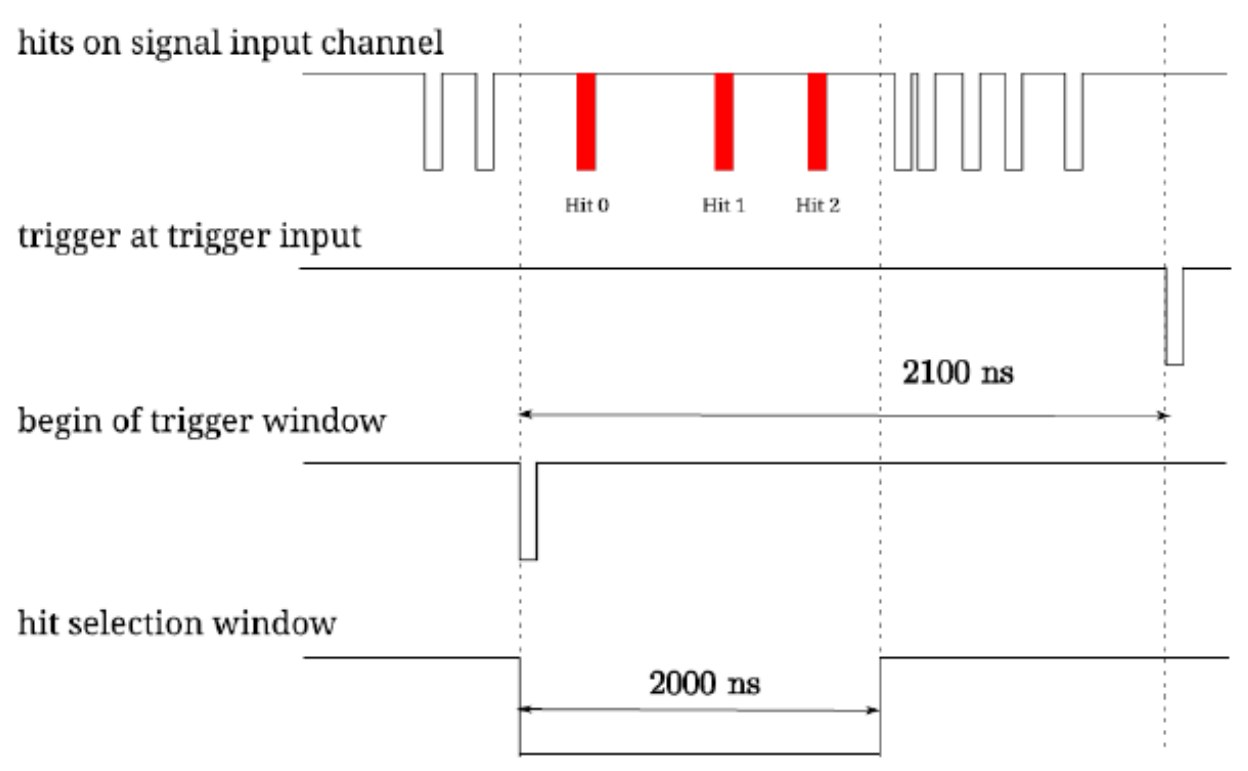

Figure 5.4: Each trigger scintillator event opened up the F1TDCs window, collecting up to 7 hits. Hits 0,1 , and 2 (red) have their times digitized. This key feature enabled investigation of detector electronic deadtime. Courtesy of [118].

\section{Rate determination and accidental correction}

The raw measured rates come from the main detector scaler banks, which are read out with the $10 \mathrm{~Hz}$ clock trigger. The number of counts for each 0.1 second window are averaged over the whole run. The average rates are determined for each main detector bar (both photomultiplier tubes, or PMTs). The number of accidentals subtracted is related to the rate of uncorrelated pulses from the individual tubes. The number of uncorrelated pulses, $R_{\text {uncorrelated }}$ for $\operatorname{PMT} j$ is given by

$$
R_{\text {uncorrelated }}^{j}=R_{\text {total }}^{j}-R_{\text {bar }},
$$

where $R_{\text {total }}^{j}$ is the total number of hits in $\operatorname{PMT} j, R_{b a r}$ is the average coincidence rate determined for that main detector, and $j$ is one of the main detector PMTs (positive or negative). The main detector discriminator output pulse width is $40 \mathrm{~ns}$, so the maximum possible coincidence width between the PMTs is $\Delta T=79 \mathrm{~ns}$. Then a firstorder estimation for the accidental contribution is

$$
R_{\text {acc }}=\left(R_{\text {uncorrelated }}^{\text {pos }}\right) \cdot\left(R_{\text {uncorrelated }}^{\text {neg }}\right) \cdot \Delta T
$$

where pos and neg denote positive and negative PMTs respectively. Other methods were also used to determine the main detector rate and accidentals. These other methods, pioneered for the event-mode analysis, provided a more robust determination of 
the accidentals contribution, which had excellent agreement with the calculation here (see Appendix B for more details). Typically, the accidentals corrections were $\mathcal{O}(2 \%)$.

\section{Electronic live time correction}

Since the dilution analysis acquires its information from the scaler banks and not the event-mode DAQ, there are no DAQ dead time or pre-scaling issues. Instead, the primary contributor of dead time comes directly from the main detector response. The main detector exhibited a pulse structure typically lasting several hundred ns. As such long pulses were unanticipated, the main detector discriminator width was set at $40 \mathrm{~ns}$. These features created long dead times that made detailed modeling a challenge.

Ideally, the detector signal is short compared to the logic signal (discriminator) output width, and the detector dead time contribution depends on the rate of the overall main detector phototubes, including both the correlated and uncorrelated pulses. Live time corrections are exponential in nature [119], so a first-order approximation which avoids double counting is:

$$
L T_{\text {elec }}=e^{R \tau} \approx 1-R_{\text {tot }} \cdot \Delta T
$$

where the dead time constant is $\tau$, the main detector's $40 \mathrm{~ns}$ discriminator width is $\Delta T$ and $R_{t o t}$ is the sum of the correlated and uncorrelated tube rates,

$$
R_{\text {tot }} \equiv R_{\text {correlated }}+R_{\text {uncorrelated }}^{\text {pos }}+R_{\text {uncorrelated }}^{\text {neg }}
$$

Here pos and neg denote positive and negative PMTs, respectively. Using equation (5.6), the naive live time correction simplifies to

$$
L T=1-\Delta T\left(R_{\text {pos }}^{\text {total }}+R_{\text {neg }}^{\text {total }}-R^{\text {correlated }}\right) .
$$

In practice, this produced incorrect results, as seen in beam current scans (see Appendix B, Figure B.1 for an example). Indeed, Figure 5.5 shows that the calculated rate at high currents on a solid target are approximately 10-15\% less than at low currents. This is well outside of any BCM uncertainty, which is $\mathcal{O}(1.5 \%)$. As will soon become apparent, the problem is the $40 \mathrm{~ns}$ discriminator width $(\Delta T)$, and the assumption the main detector pulse is short compared to the discriminator width.

The multi-hit capabilities of the F1TDCs, discussed in Section 5.2.1, enabled indepth study of detector pulse structures, such as the double pulse resolutions seen in Figures 5.6 (trigger scintillator) and 5.7 (main detectors, Run 1 and Run 2). The double pulse resolution is the minimum time between successive pulses that the DAQ can resolve. 


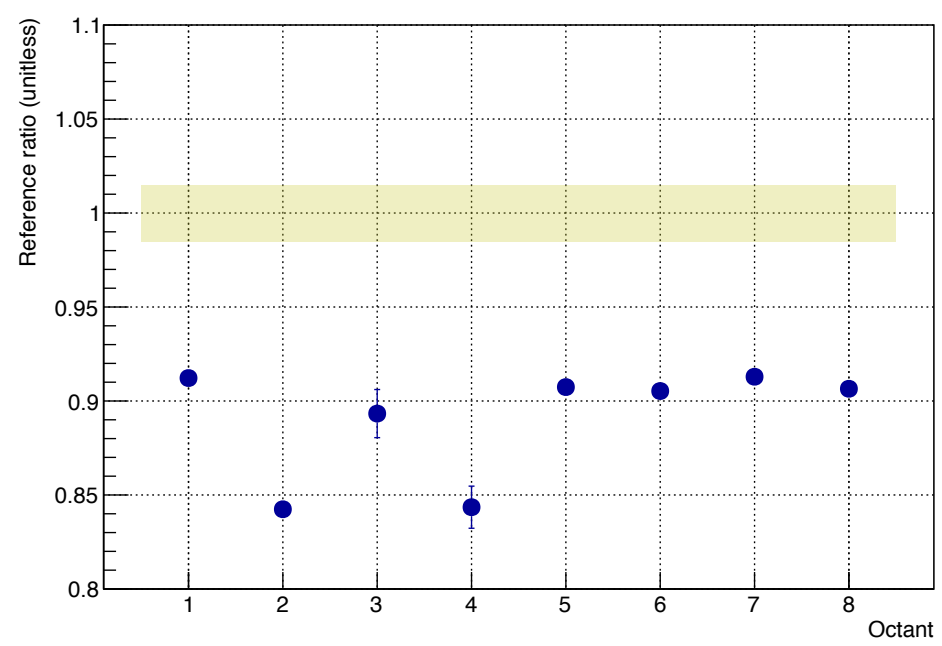

Figure 5.5: Ratio of extracted rates on a solid carbon target at $1 \mu A$ and $100 \mathrm{nA}$. The ratio $Y_{1 \mu \mathrm{A}} / Y_{100 \mathrm{nA}}$ is expected to be $1.00 \pm 0.15$, due to expected BCM uncertainties (shaded yellow box). The significant disagreement with expected values is due to using an inappropriate characterization of the main detector's electronic dead time. The uncertainties, including statistics, and corrections for non-current systematics are within the plotting symbols, except for octants 3 and 4 which experienced excessively noisy tubes.

Recall the trigger scintillator has short output signals of a few ns [42], but its discriminator has an output width of $142 \mathrm{~ns}$, producing the deterministic double pulse separation seen in Figure 5.6. ${ }^{5}$ However, the main detectors had long pulses and a short discriminator width. From Figure 5.6, note the sharp rise around 140 ns, corresponding to the logic unit (discriminator) width. The probability of successive hits is constant (from Poisson statistics); however, with each new hit the size of the remaining event window falls, and the probability of a subsequent hit being detected within that time also decreases, hence the fall-off for $\Delta T>200 \mathrm{~ns}$.

Figure 5.7 shows the main detector double pulse resolution, which displays many interesting structures. A hard-cutoff at $40 \mathrm{~ns}$ corresponds to the physical minimum pulse separation allowed by the discriminator. In Run 1, there are few events with time separations less than $200 \mathrm{~ns}$, and a sharp step-function-like increase around 300 ns, corresponding to the typical double pulse resolution. This sharp rise in Run 1 generally made determining the double pulse resolution straight-forward. Run 2 is less clear, exhibiting a steady rise time. This is suggestive that the double pulse resolution depends on pulse-height, with smaller pulses allowing earlier subsequent hits after larger

\footnotetext{
${ }^{5}$ The excessively long discriminator dead time was a historical bug that turned into a feature: it was hoped to provide the limiting dead time component, making calculations and corrections easy and conclusive in the event-mode analysis.
} 
Time Between Successive TS Hits (Run 2)

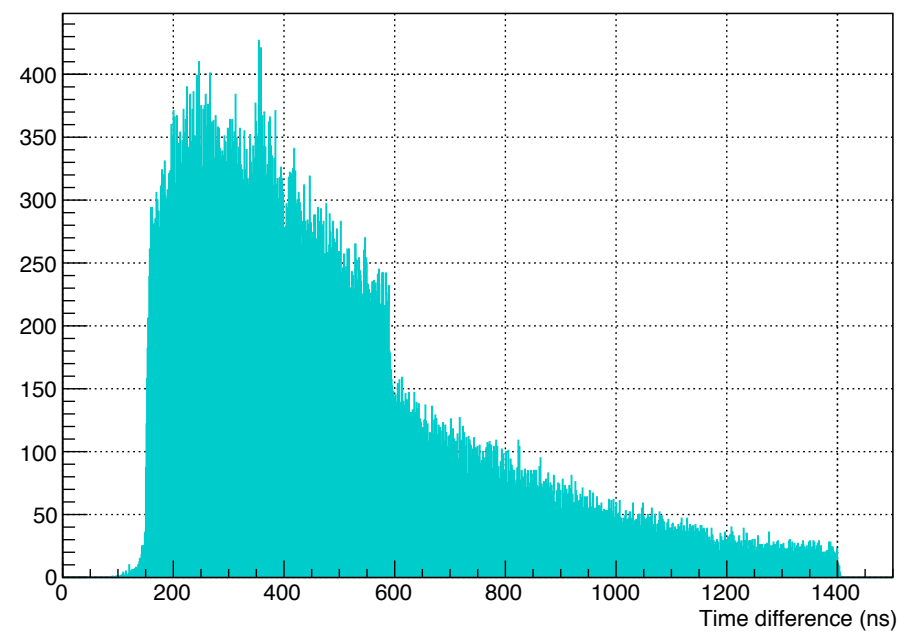

Figure 5.6: Time difference between successive pulses in the trigger scintillator.

Time Between Successive MD Hits (Run 1)

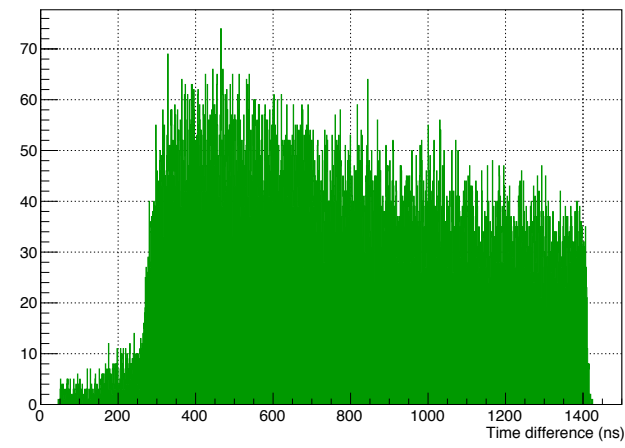

Time Between Successive MD Hits (Run 2)

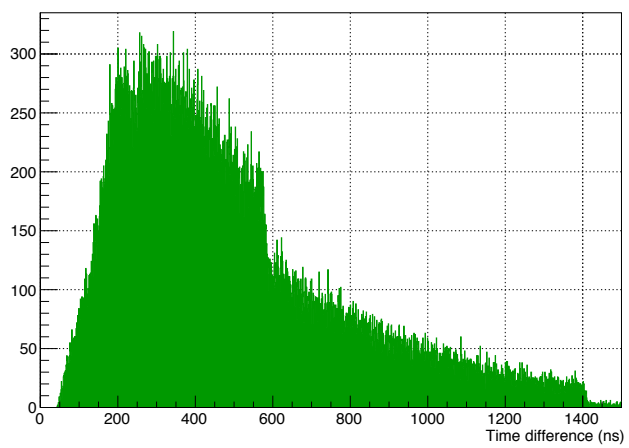

Figure 5.7: Typical main detector double pulse resolutions for Run 1 (left) and Run 2 (right). Both show octant 1, with $1 \mu A$ of beam on a solid Carbon target. Run 1 used a $0.5 \%$ radiation length target and had $\sim 300 \mathrm{kHz}$ coincidence rate. Run 2 used a $1.6 \%$ radiation length target, and had rates of $\sim 1250 \mathrm{kHz}$. 

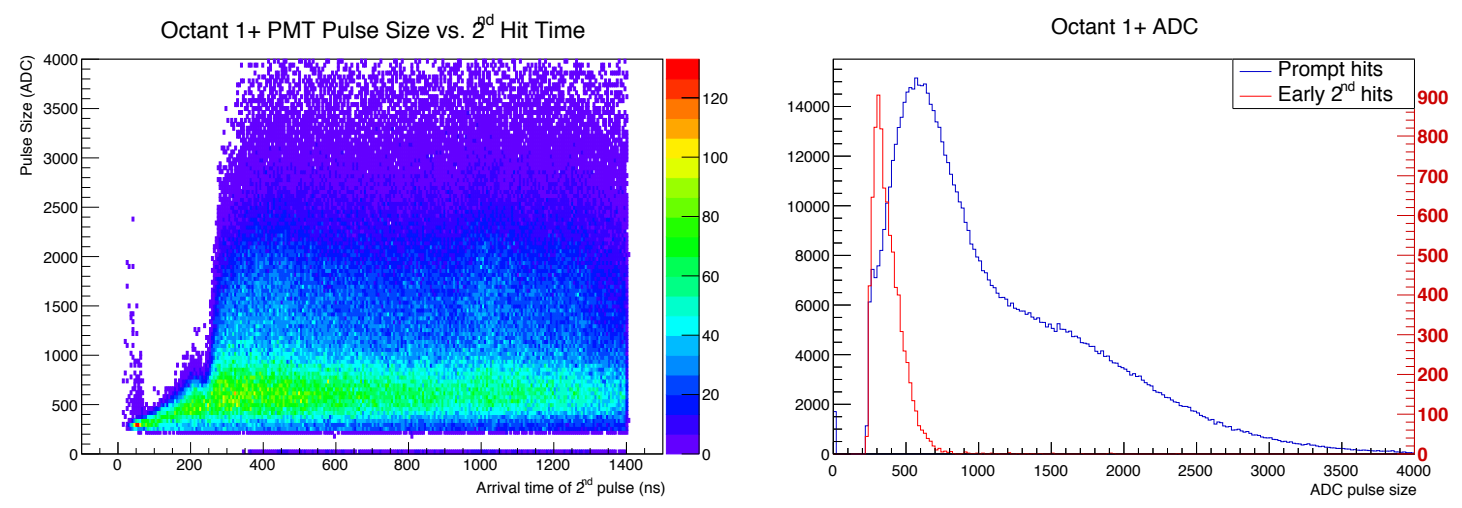

Figure 5.8: (Left) Main detector pulse size (ADC) versus second hit arrival time. (Right) The pulse size (ADC) spectra for prompt hints (blue, left axis) and prompt hits with early second hints (red, right axis). Early second hits are those detected within $200 \mathrm{~ns}$ of the initial trigger.

pulses. The grass seen early in Run 1 is presumably from such small pulses.

Figure 5.8 (left) shows evidence of a pulse-height dependent dead time. A clear correlation exists between the initial pulse size and recorded arrival time of subsequent hits. Only the smallest initial pulses allow second hit times between 40-200 ns later, while the largest initial pulses don't allow second pulses for almost 300 ns. Figure 5.8 (right) shows the pulse size for all prompt pulses in blue; those events with early second hit times are shown in red. Clearly only the smallest pulses allow re-triggering within a few hundred ns after the initial pulse.

From Figures 5.7 and 5.8, an informative picture emerges of the problem. Our main detectors are long, thin Cherenkov detectors; although direct, line-of-sight photons from a track at one end might only take $\sim 10$ ns to reach the opposite PMT, many photons will ultimately take much longer. The tracking mode DAQ was designed to see every real event, so the discriminator thresholds were set low ( $<1$ photoelectron). With each elastic electron generating approximately 50-100 photoelectrons [93], the end result is a long pulse structure and pulse-height dependent electronic dead time. That is, larger pulses take longer to fall below the discriminator threshold. Thus, the discriminator is no longer the defining component of dead time; it only defines the minimum deadtime of $40 \mathrm{~ns}$.

Since each detector's threshold may vary from the others this correction is also octant and detector dependent. Incidentally, this also presumably explains why the double pulse resolution looks so different between Runs 1 and 2: the main detector thresholds were set lower in Run 1, resulting in longer dead times.

Using this interpretation, determining the appropriate double pulse resolution was 
fairly straightforward ${ }^{6}$ Runs where the main detector acted as the trigger were used, as they would generate sufficient statistics quickly. ${ }^{7}$ Figures 5.9 and 5.10 are examples for Runs 1 and 2, respectively, for all eight main detectors. The double pulse resolution was defined to be the first time where the value was half of the maximum value. This value was initially chosen as a rough approximation for the average double pulse resolution.

The histogram bin size was varied to see the effect of the calculated double pulse resolution, using this first bin of half maximum model. These binning effects were found to be fairly small $(\mathcal{O}(8 \mathrm{~ns}))$, so a conservative $10 \mathrm{~ns}$ uncertainty was assigned for this. Next, during Run 2 multiple runs under identical conditions were performed during several tracking periods, and the results were reproducible to within $10 \mathrm{~ns}$ (Table 5.1). The data were taken once at the beginning of Run 2 (November 2011, Run 13714), twice in the middle of Run 2 (January 2012, Runs 15034 and 15123), and once at the end of Run 2 (May 2012, Run 18580). The May data exhibited radically different behavior and some signs of gain/threshold variation. Since all of the relevant Run 2 dilution measurements were taken during the same January 2012 period, the average of only these results were used, with a conservative 10 ns uncertainty applied.

The largest uncertainty comes from the choice of model, that is the choice to define the double pulse resolution value at half of the peak. Admittedly, this choice is somewhat arbitrary, although its use removes the current dependence seen in Figure 5.5 reasonably well. It seems unlikely that the extracted double pulse resolutions could be off by more than $30 \mathrm{~ns}$ ( $\sim 20 \%$ of the Run 2 value), so this is taken as an additional model uncertainty. The total uncertainty for the electronic live time would be \pm 33 ns (the quadrature sum of binning, averaging, and model uncertainties). Assuming a nominal 150 ns double pulse resolution in Run 2, this is a $\sim 20 \%$ relative uncertainty. For Run 1, a 30 ns model uncertainty was also used, which is a $10 \%$ relative uncertainty, assuming the average double pulse resolution was $\sim 300 \mathrm{~ns}$. This seems appropriate, given that the Run 1 double pulse resolution was much better defined in Run 1 . The uncertainty of this model dependence on the calculated dilution was empirically determined by observing how the calculated dilution changed with a $\pm 30 \mathrm{~ns}$ shift in the double pulse resolution.

\footnotetext{
${ }^{6}$ If the deadtime were strictly due to electronics, a simple Monte Carlo model would suffice; but building a realistic Monte Carlo incorporating varying pulse sizes would require lots of input information. It is much easier to simply measure it.

${ }^{7}$ Compared to the usual trigger scintillator runs.
} 

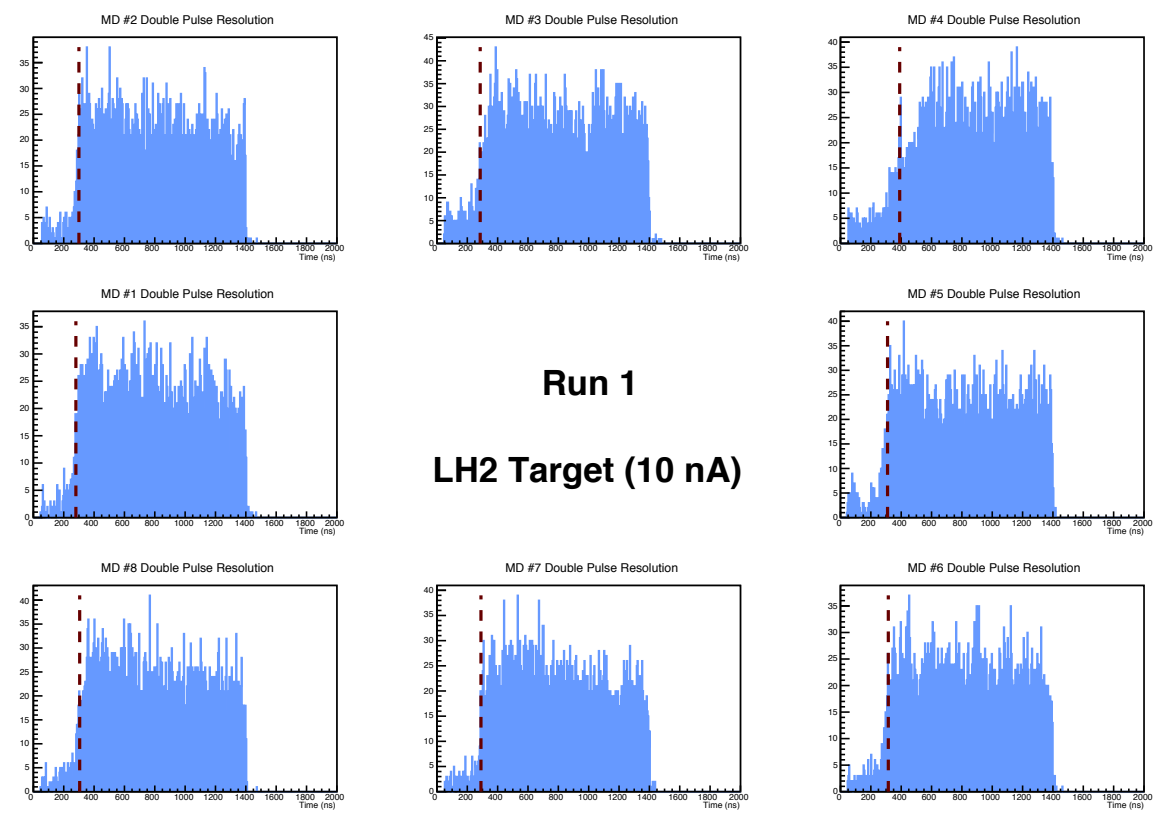

Figure 5.9: Double pulse resolutions, by octant, for $Q_{\text {weak }}$ Run 1. The red line is the determined double pulse resolution for that detector.
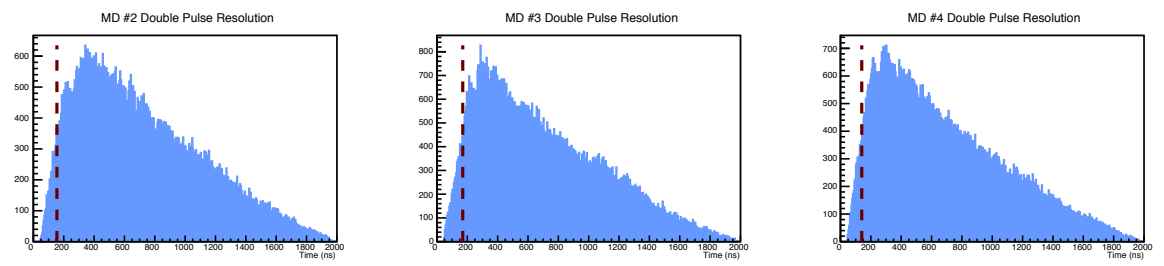

MD \#1 Double Pulse Resolution
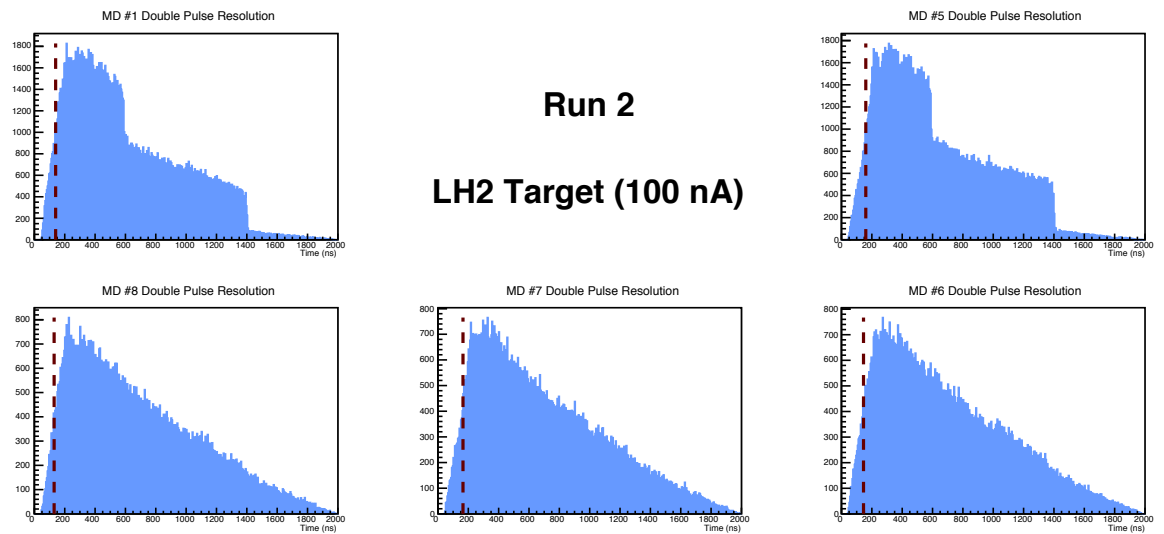

Figure 5.10: Double pulse resolutions, by octant, for $Q_{\text {weak }}$ Run 2. The red line is the determined double pulse resolution for that detector. Note that there is a different structure in Run 2 octants 1 and 5 than seen in Run 1 (Figure 5.9). This is an ancillary effect due to the main detector 1 and 5 trigger, and is not well understood. 


\begin{tabular}{|c|c|c|c|c|c|}
\hline \multirow{2}{*}{ Octant } & Run 1 & \multicolumn{5}{|c|}{ Run2 } \\
\cline { 2 - 6 } & 10584 & 13714 & 15034 & 15123 & 18580 \\
\hline 1 & 280 & 135 & 140 & 140 & 115 \\
\hline 2 & 300 & 145 & 145 & 155 & 125 \\
\hline 3 & 285 & 150 & 160 & 170 & 155 \\
\hline 4 & 390 & 140 & 130 & 140 & 100 \\
\hline 5 & 310 & 165 & 155 & 160 & 140 \\
\hline 6 & 315 & 145 & 140 & 145 & 105 \\
\hline 7 & 290 & 155 & 155 & 165 & 140 \\
\hline 8 & 305 & 140 & 140 & 130 & 115 \\
\hline
\end{tabular}

Table 5.1: Double pulse resolutions determined for both Runs 1 and 2, in ns. Since the dilution data was taken in January 2012, the average of the January numbers were used (Runs 15034 and 15123).

\section{Beam current determination}

To compare with simulation, absolute determination of the beam current is needed. The Run 1 final BCM calibration analysis was discussed in [42]; in Run 2, a beam current monitor BCM calibration was done in early February 2012 at low-current for this purpose. The calibration covered current ranges from approximately $50 \mathrm{nA}$ to $5 \mu \mathrm{A}$, although the BCMs demonstrated non-linear behavior at the edges of this range. Instead, the results used here were based on data between $70 \mathrm{nA}$ and $2 \mu \mathrm{A}$.

A Faraday Cup (FC) located in the $5 \mathrm{MeV}$ region of the injector was used to measure the beam current, while the BCM response was recorded from their scalers and were calibrated against the FC afterwards. The statistical uncertainty of the BCM was taken as the distribution width instead of the error on the mean $(\mathrm{RMS} / \sqrt{N})$, to be conservative, and was sub-percent. The statistical uncertainty in the FC measurements was likewise small, typically sub-percent.

The important systematic uncertainties for the Faraday cup are position sensitivity, secondary electron emission, backscattering, absolute ammeter calibration, and current loss during transport. The beam position sensitivity was measured in Run 1, and found to be a $\sim 1 \%$ effect. During Run 2, similar results were obtained. The contributions from secondary emission, backscattering, and transport loss were bounded in Run 1 by a high-current calibration of the FC to the Hall C Unser monitor [42]. The difference (FC - Unser) at $150 \mu \mathrm{A}$ was found to be $(-1.4 \pm 0.3) \%$. For this analysis, we take the total uncertainty as $1.4 \%$. This estimate is likely overly conservative, as current loss during transport due to space charge effects is likely the dominant contributor at $150 \mu A$. Such effects are current dependent, and should be much smaller between 0.1 and $1 \mu A$, where this calibration was performed. The other uncertainty contributions, although current 

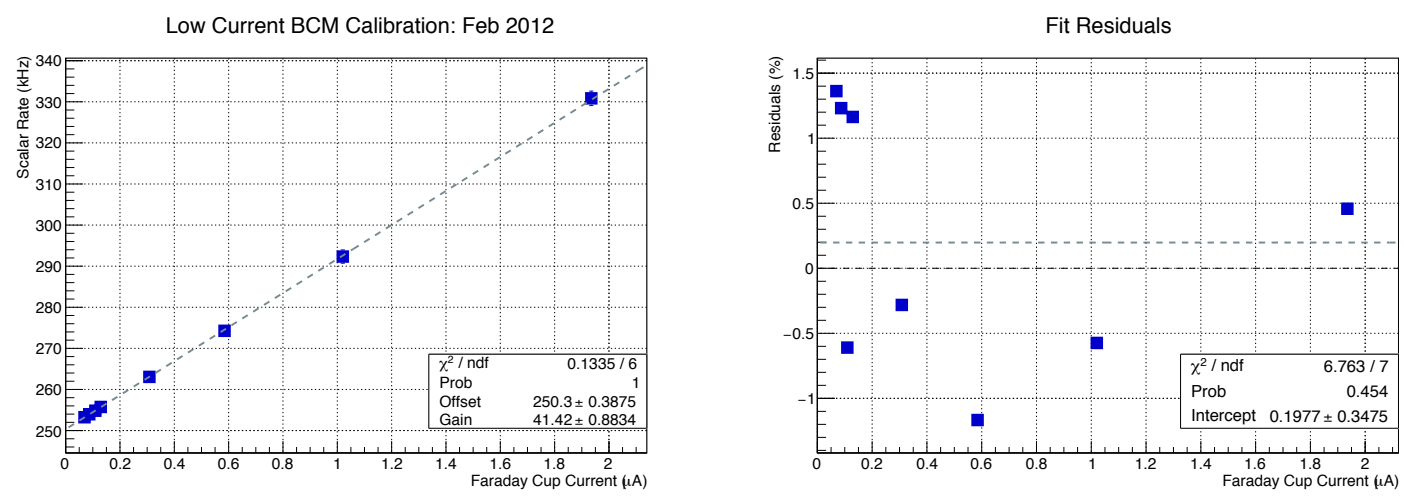

Figure 5.11: Run 2 low-current BCM calibration. (Left) BCM scaler fit to data. (Right) Fit residuals in percent $\left(\left(I_{\text {true }}-I_{\text {fit }}\right) / I_{\text {true }}\right)$.

independent, are likely small in comparison.

The BCM scaler rates were extracted in $\mathrm{kHz}$ and fit to a first-order polynomial. Figure 5.11 shows the final fit between $70 \mathrm{nA}$ and $2 \mu \mathrm{A}$. The current, $I$, can be extracted from the $\mathrm{BCM}$ rate, $R_{B C M}$ from

$$
I=\left(R_{B C M}-b\right) / g
$$

where $b$ is the fit offset in $\mathrm{kHz}$ and $g$ is the gain (fit slope) in units of $\mathrm{kHz} / \mu A$. The dilution data were all taken at $\sim 100 \mathrm{nA}$ or $1 \mu \mathrm{A}$, where the residuals are about $0.5 \%$, well within the FC position sensitivity uncertainty. With such fit residuals and small position drifts, the final BCM uncertainty is given as 1.5\%.

Beyond the calibration, all dilution data underwent strict quality control. All beam trips were excluded, and only portions of runs with stable beam current of $\pm 1 \%$ were kept. Runs, or run segments, not within these bounds were discarded.

\subsubsection{Reference target normalization}

Data taken on solid dummy targets at several currents provided an excellent way to check the efficacy of our corrections, in particular the dead time. A more effective approach would be a proper current scan, where one measures the scattering rate of a dummy target at several different currents. During $Q_{\text {weak }}$, running on solid carbon targets only happened at $\sim 80 \mathrm{nA}$ and $1 \mu \mathrm{A}$, as these were the currents at which most dilution measurements were performed.

Originally the purpose of these solid target measurements was to reduce the dependence on the BCMs, which are potentially non-linear over the desired current range. An improved determination of the dilution could account for such effects by forming a 
super-ratio

$$
f_{\text {ref }}=\frac{\left(Y_{\text {empty }} / Y^{r e f}\right)_{1 \mu A}}{\left(Y_{\text {full }} / Y^{r e f}\right)_{0.1 \mu A}}=\frac{Y_{\text {empty }}}{Y_{\text {full }}} \cdot\left(\frac{Y_{0.1 \mu A}^{r e f}}{Y_{1 \mu A}^{r e f}}\right),
$$

where $Y_{\text {empty }}\left(Y_{\text {full }}\right)$ are the current-normalized rates on an empty (full) hydrogen target, $Y_{\text {ref }}$ are the current-normalized rates on a solid Carbon dummy target, and the second term in parenthesis is a correction factor for any rate-dependence in the reference normalization and is ideally $\approx 1$. Once again, this could be from any ratedependent effect, but was originally conceived to mitigate potential non-linearities in the BCM electronics chain. To first order all the BCM systematics would drop out, and their relative contribution to the dilution would be small.

Two reference target runs were used in the both Run 1 and Run 2 analysis, one each at high and low current. Table 5.2 shows the average rates and corrections sizes, in percent, for both of these runs. Although the high-current runs were taken at similar beam currents, the measured Run 2 rates were over 4 times larger than measured during Run 1 . This is because the Run 1 target was a $0.5 \%$ radiation length thick target, while in Run 2 it was a $1.6 \%$ radiation length target.

\begin{tabular}{|l|c|c|c|c|}
\hline \multirow{2}{*}{ Run } & \multicolumn{2}{|c|}{ Run 1 } & \multicolumn{2}{c|}{ Run 2 } \\
\cline { 2 - 5 } & 10709 & 10791 & 14938 & 15130 \\
\hline Current $(\mu A)$ & 0.9967 & 0.1195 & 1.1570 & 0.0705 \\
\hline Rate $(\mathrm{kHz})$ & 317 & 39.6 & 1408 & 89.2 \\
\hline Accidentals $(\%)$ & -0.62 & -1.07 & -2.5 & -0.3 \\
\hline Deadtime (\%) & 4.51 & 0.65 & 17.6 & 1.3 \\
\hline
\end{tabular}

Table 5.2: Reference target (Carbon) data by run.

Sadly, as the rates at high-current were excessively large, the model-dependent dead time uncertainties were overwhelming, leading to the super ratio solid target normalization approach being abandoned. However it still has merit as it can show the overall effectiveness of the rate dependent corrections. Figure 5.12 shows the ratio of $\left(Y_{0.1 \mu A}^{r e f} / Y_{1 \mu A}^{r e f}\right)$ versus main detector octant. In Run 2, the electronic deadtime model uncertainty is prodigious; it's approximately $4.5 \%$, which is too large for the $Q_{\text {weak }}$ result. Therefore the reference target normalization method was dropped from the final $Q_{\text {weak }}$ analysis. Although an acceptable method in Run 1, it was dropped for both runs for simplicity and consistency in the analysis.

Although unable to constrain BCM uncertainties, this analysis does suggest our corrections are appropriately sized. Even with the large uncertainty, the mean values of the corrected rates are within a few percent of unity. If, for example, our accidental corrections or deadtime model were significantly incorrect, the mean values would be 

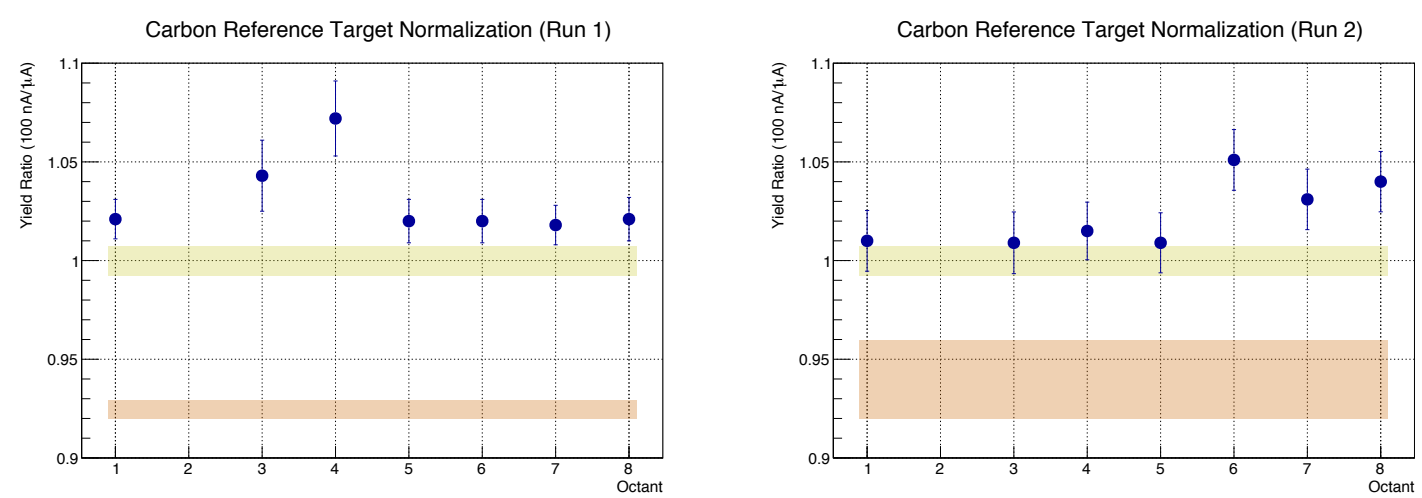

Figure 5.12: Ratio of current-normalized rates on solid carbon target. The ratio is expected to be identically 1.00. The yellow band is a 1.5\% BCM uncertainty, while the orange band is the effect of the dead time model uncertainty. This model uncertainty is found by shifting the double pulse resolution by its uncertainty. Octant two is disregarded in both cases due to known hardware issues discussed in Section 5.3.

farther from unity.

\subsection{Data and analysis}

Running on an evacuated target occurred only once during each $Q_{\text {weak }}$ run period; additional measurements were forgone to minimize risk of target damage. In Run 1, there were two measurements on an evacuated target (Runs 10717 and 10719) and one measurement taken on a target full of $\mathrm{LH}_{2}$ (Run 10798). Figure 5.13 gives the current-normalized rates measured by octant for each run.
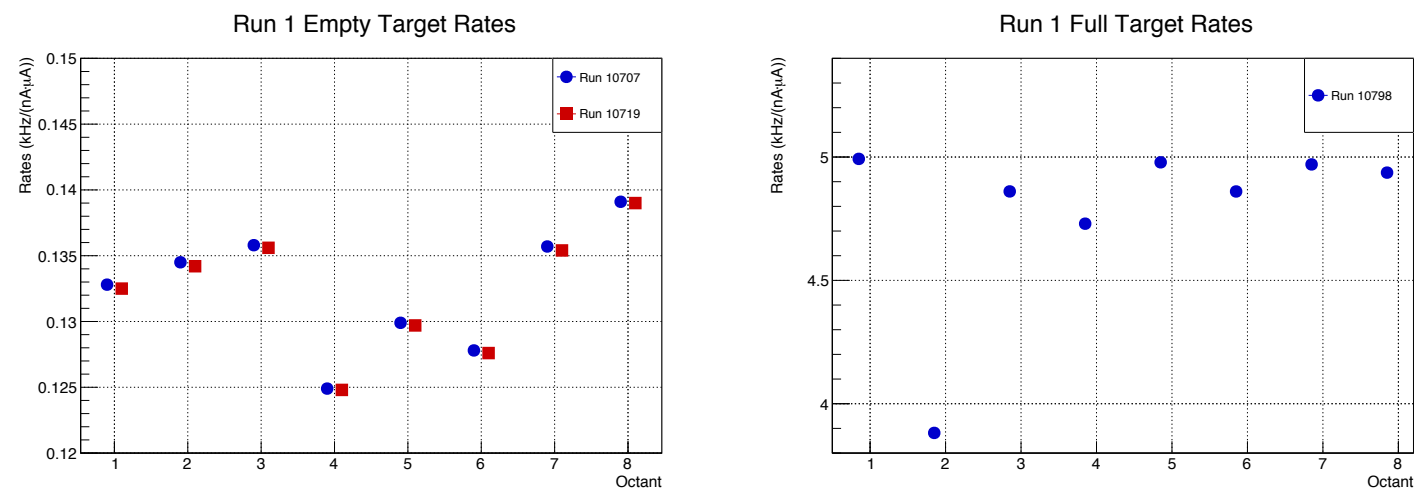

Figure 5.13: Measured rates on both the evacuated and full target in Run 1. The points include statistical uncertainty bars but are smaller than the plotting symbols. Note the octant 2 discrepancy at high rate (full target). This is presumably due to gain sagging.

During Run 2 the full set of dilution measurements were also taken once. There were four empty target runs $(14909,14915,14916,14919)$, each with the tracking detectors (HDCs, VDCs, and trigger scintillators) in the four different positions. This enabled 

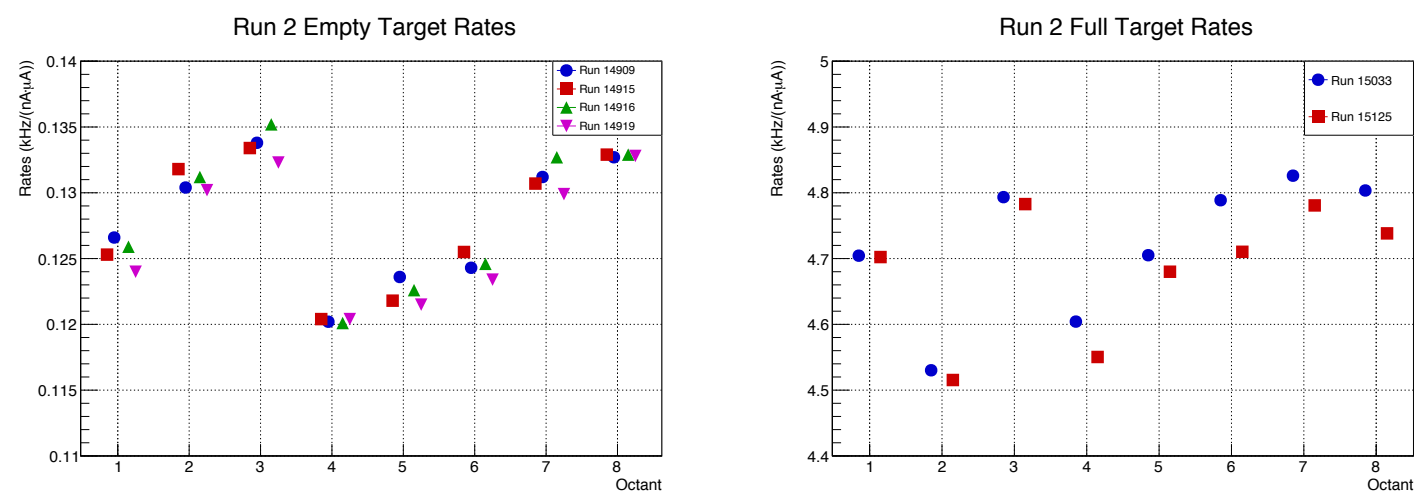

Figure 5.14: Measured rates for the evacuated and full targets for Run 2, by octant. The points include statistical error bars added in quadrature with the accidental correction uncertainty. Like in Run 1 , octant 2 appears to have additional rate dependent problems, as is evidenced by the relative drop during full target (high rate) running compared to the other octants. For the final data set, Run 15125 was removed; it is included here for completeness, and to show reasonable consistency with Run 15033.

alternate rate calculation methods using the trigger scintillator, as well as the ability to finely explore octant dependent variations in the scattering profile. Four runs were also taken during this period on the full target $(15033,15108,15115$, and 15125), but incorrect settings with the trigger supervisor prescale eliminated 15108 and 15115 as useful. Run 15125 had large beam current drifts, and the only stable current period averaged $\sim 80 \mathrm{nA}$; it was preferred to keep the current above this due to the large BCM residuals seen in Figure 5.11. Run 15033 had a stable period with average current of $\sim 100 \mathrm{nA}$ and no DAQ prescale problems, so was ideal for the Run 2 dilution analysis. Figure 5.14 provides the Run 2 current-normalized rates for the evacuated and full target running. Table 5.3 gives a summary of the average rates and corrections for the dilution runs.

\begin{tabular}{|l|c|c|c|c|}
\hline \multirow{2}{*}{ Type } & \multicolumn{2}{|c|}{ Run 1 } & \multicolumn{2}{c|}{ Run 2 } \\
\cline { 2 - 5 } & Empty & Full & Empty & Full \\
\hline Current $(\mu A)$ & 0.9982 & 0.1190 & 1.130 & 0.1019 \\
\hline Rates $(\mathrm{kHz})$ & 132 & 568 & 144 & 481 \\
\hline Accidentals $(\%)$ & -0.64 & -1.94 & -0.14 & 3.75 \\
\hline Deadtime $(\%)$ & 4.0 & 15.2 & 2.1 & 6.7 \\
\hline
\end{tabular}

Table 5.3: Summary of average rates and correction sizes for the dilution analysis. Both Run 1 and Run 2 averages are given here. These numbers include all eight octants, even though there are strong reasons why certain octants should be discarded, as discussed in the text.

Figures 5.13 and 5.14 both show changes in the octant dependence from low to high rate running (i.e., the shape of the variation). In Run 1 this was due to hardware 

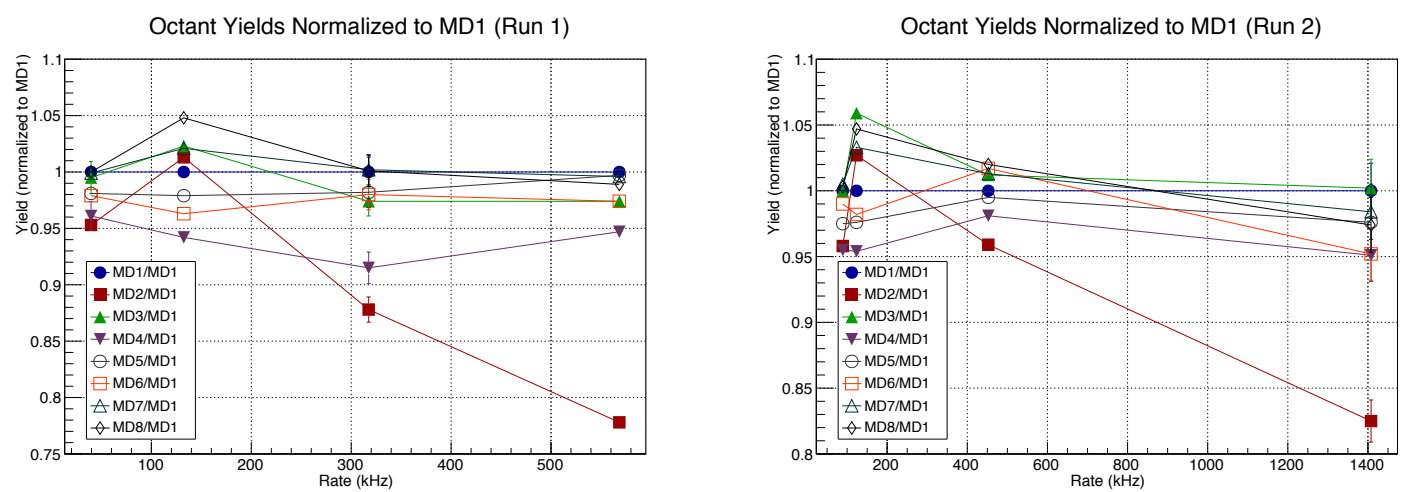

Figure 5.15: Measured rates for each octant normalized to main detector 1 . In both Run 1 (left) and Run 2 (right) main detector 2 shows clear evidence of problems; with increased rate main detector 2's response drops quickly. This is most pronounced in Run 1, whereas this appears to only be a problem at very large rates in Run 2. For these reasons Octant 2 will be discarded from the final Run 1 aluminum target dilution analysis.

problems such as noisy tubes and gain sagging [42]. Figure 5.15 shows the measured rates of each octant normalized to main detector 1, and that octant 2 in particular has serious problems. Octant 2 will be discarded in Run 1 for this reason. However, Table 5.3 shows that the average rate on the Run 2 full target is $\sim 500 \mathrm{kHz}$, and these results appear consistent with the other low rate measurements. Therefore in Run 2 octant 2 data is discarded from the high-rate reference target normalization, but kept for the regular dilution analysis.

There is one final potential systematic shift using an empty target, which is thermal expansion of the thin target walls due to beam heating. Thermal expansion could potentially lead to changes in the target's areal density during evacuated target running, an effect suppressed with addition of liquid hydrogen. Computational fluid dynamics simulations determined that the beam raises the target cell walls $\sim 10 \mathrm{~K} / \mu A$, where the main heat dissipation mechanism come through conduction in the walls (it was difficult to simulate the complicated target geometry of the whole recirculation loop). Although more rigorous calculations are possible, it is simple to show the effect of any areal density changes is negligible. The coefficient of linear expansion for aluminum 7075-T6, $d L / L$, is $25 \times 10^{-6} \mathrm{~m} / \mathrm{K}$ at room temperature, suggesting a relative change in areal density of only $2 \times(d L / L) \sim 0.05 \%$. From the Third Law of Thermodynamics, $d L / L$ decreases as temperature decreases. Since the operating temperature of the empty target is between 30-40 K [120], this is a large upper bound.

The final ratio of empty target to full target current-normalized rates are shown 

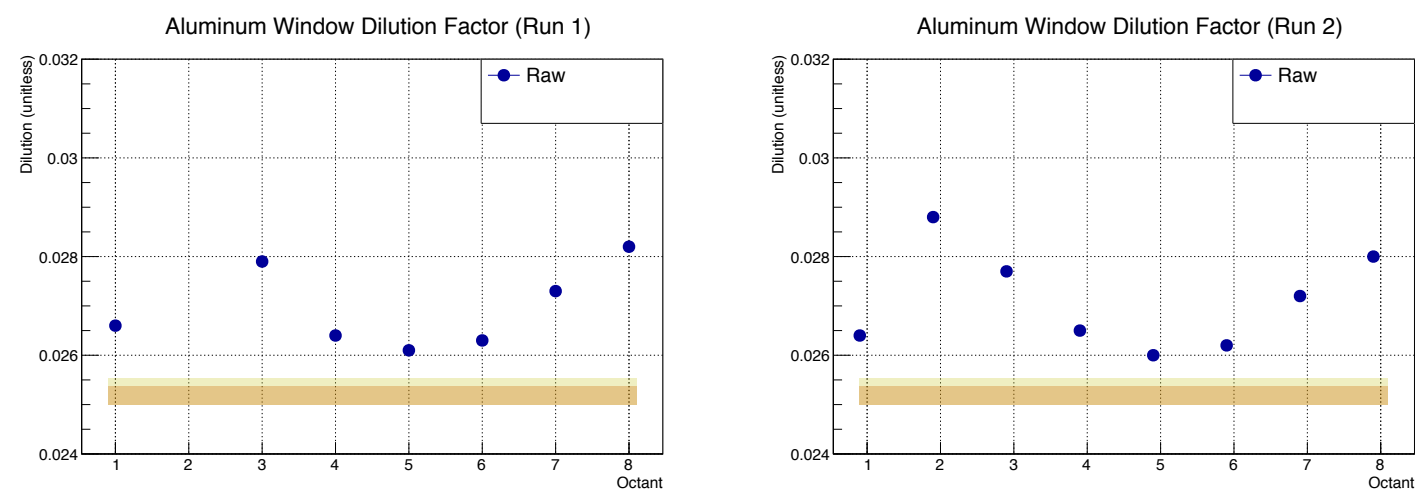

Figure 5.16: Calculated dilution factor, by octant, for both Run 1 and Run 2. Raw denotes corrections for accidentals and deadtime, but not higher-order radiative corrections. Statistical uncertainties are plotted, but are smaller than the points. Octant 2 is discarded in the Run 1 data. The orange band is the deadtime model uncertainty, while the yellow-band is the model uncertainty and $1.5 \% \mathrm{BCM}$ uncertainty quadrature sum.

\begin{tabular}{|c|c|c|c|c|c|}
\hline & $\begin{array}{c}f_{\text {average }} \\
\left(\times 10^{-2}\right)\end{array}$ & $\begin{array}{c}\text { stat+sys } \\
\left(\times 10^{-5}\right)\end{array}$ & $\begin{array}{c}\text { deadtime model } \\
\left(\times 10^{-4}\right)\end{array}$ & $\begin{array}{c}\text { BCM } \\
\left(\times 10^{-4}\right)\end{array}$ & $\begin{array}{c}\text { Total } \\
\left(\times 10^{-4}\right)\end{array}$ \\
\hline Run1 & 2.695 & 4.379 & 4.043 & 4.043 & 5.734 \\
\hline Run2 & 2.679 & 7.532 & 4.018 & 4.018 & 5.732 \\
\hline
\end{tabular}

Table 5.4: The octant-averaged dilution for Runs 1 and 2, with uncertainties. The (stat+sys) column is the contribution due to statistics and accidental corrections. The deadtime model and BCM uncertainty are both assessed at $1.5 \%$.

in Figure 5.16. Octant 2 is discarded due to the aforementioned rate dependence in Run 1. The deadtime model uncertainty (orange band) was empirically determined by noting the change in calculated dilution after shifting the double pulse resolution by its uncertainty ( $\pm 30 \mathrm{~ns}$ ). The yellow band is the quadrature sum of the model-uncertainty and the nominal 1.5\% BCM uncertainty.

The octant-averaged dilutions with uncertainties are given in Table 5.4. The decision to average over the octants was made to simplify the final $Q_{\text {weak }}$ analysis, since no other background corrections were made in an octant-dependent manner. Different methods of averaging (unweighted vs. weighting by the individual octant main detector asymmetry width) were investigated and found to vary less than a percent. Ultimately the simple arithmetic mean was used.

\subsection{Radiative corrections}

The dilution factor requires higher-order corrections. To minimize the upstream window contribution, the scattering profile was designed to fall slightly off the bar. When liquid 
hydrogen is added to the target for the full measurements, increased radiative losses, bremsstrahlung, and multiple scattering shift the upstream window profile onto the bar. The downstream window profile, although sitting square on the bar, does experience a related shift which is purely kinematic (i.e., a change in the average $Q^{2}$ ).

Determining these higher-order corrections requires simulation, as there are no experimental techniques to separate the scattering off of the individual upstream and downstream windows. Some progress has been made using the drift chambers to perform such an analysis, but is of modest statistical power and is not discussed further. Applying higher-order corrections is an intricate process, and it helps by separating components into individual upstream and downstream window scattering rates as $Y_{U S}$ and $Y_{D S}$ respectively. For instance, one can re-cast the raw dilution calculated previously as

$$
f_{\text {raw }}=\frac{Y_{\text {empty }}}{Y_{\text {full }}}=\frac{Y_{U S}+Y_{D S}}{Y_{U S}+Y_{D S}+Y_{L H 2}} .
$$

The individual upstream and downstream window empty target rate fractions can be defined as

$$
f_{U S} \equiv \frac{Y_{U S}}{Y_{U S}+Y_{D S}} \quad f_{D S} \equiv \frac{Y_{D S}}{Y_{U S}+Y_{D S}},
$$

with the important constraint

$$
f_{U S}+f_{D S}=1
$$

A radiatively corrected dilution factor, $f^{r a d}$, can then be defined as

$$
f^{r a d}=\left(C_{U S}^{r a d} f_{U S}+C_{D S}^{r a d} f_{D S}\right) f^{r a w},
$$

where $C_{U S}^{r a d}$ and $C_{D S}^{r a d}$ are the individual upstream and downstream window radiative correction factors. Both the individual window fractions, $f_{U S}$ and $f_{D S}$, and the correction factors, $C_{U S}^{r a d}$ and $C_{D S}^{r a d}$, come from simulation. The correction factors are simply the simulation's predicted ratio of scattering rate for the individual windows of a full target with that of an empty target:

$$
C_{U S / D S}^{r a d}=\frac{Y_{U S / D S}^{s i m}(\text { full })}{Y_{U S / D S}^{\text {sim }}(\text { empty })} .
$$

The average empty target rate fractions and correction factors determined by GEANT4 [121] are given in Table 5.5. For the final analysis, the correction factors were determined for each octant and applied individually before taking the octant average to account for any potential octant-dependent effects. This analysis was done with the Run 2 simulation, but should also provide an acceptable estimate of the Run 1 correction. 


\begin{tabular}{|c|c|c|c|c|}
\hline$f_{U S}(\%)$ & $C_{U S}^{r a d}$ & $f_{D S}(10 \%)$ & $C_{D S}^{r a d}$ & $\left(f_{U S} C_{U S}^{r a d}+f_{D S} C_{D S}^{r a d}\right)$ \\
\hline 45.4 & 1.20 & 54.6 & .957 & 1.065 \\
\hline
\end{tabular}

Table 5.5: Octant-averaged correction factor values.

\subsubsection{Simulation model uncertainties}

Since these additional radiative effects shift the upstream window scattering profile onto the main detector focal plane, one way to assess the simulation's validity would be seeing how the detector rates vary as a function of the spectrometer magnet's (QTOR) magnetic field. Increased magnetic field adds more bending or deflection to the elastically scattered electrons, increasing the percentage of the elastically scattered profile on the main detector focal plane; decreased magnetic field would likewise reduce the deflection, lessening the amount of the scattering profile on the detector focal plane. Comparison of predicted rates with measurements during such QTOR scans, where the magnetic field was changed, should accurately gauge the simulation's accuracy in treating such energy changes.

QTOR scans were conducted in both Run 1 and Run 2, however the data shown here are from Run 1 only. Both upstream 1\% radiation length (US-1\%) and downstream 4\% radiation length (DS-4\%) solid targets were used for these studies. Figure 5.17 shows the absolute rates during QTOR scans with the upstream (left) and downstream (right) solid targets. Measured data are represented by blue circles, while open-red squares show the predicted rates from simulation. The nominal QTOR setting where the elastic rate is maximized is at $8921 \mathrm{~A}$. Note that the downstream target rate is essentially flat at fields larger than this point, presumably because the scattering profile sits squarely on the detector focal plane and is thus relatively insensitive to changes in the magnetic field.

Note also that although the overall scale is off, the downstream variation closely matches the data for currents $i 8800 \mathrm{~A}$. Figure 5.18 shows this by plotting the relative rate change comparison to the rate at the nominal setting for both upstream (left) and downstream (right) targets. Notice that the downstream target shows excellent agreement; the upstream window shows reasonable agreement in the vicinity immediately around the peak, but shows larger disparity beyond a 1\% change in field.

Figure 5.19 shows the percent difference between the measured and simulated rates as a function of QTOR current. As noted previously, the relative rate difference is at the percent level around the elastic peak, but quickly grows. The disagreement is approximately $10 \%$ for a $1 \%$ shift in QTOR, and approximately doubles with an increase 

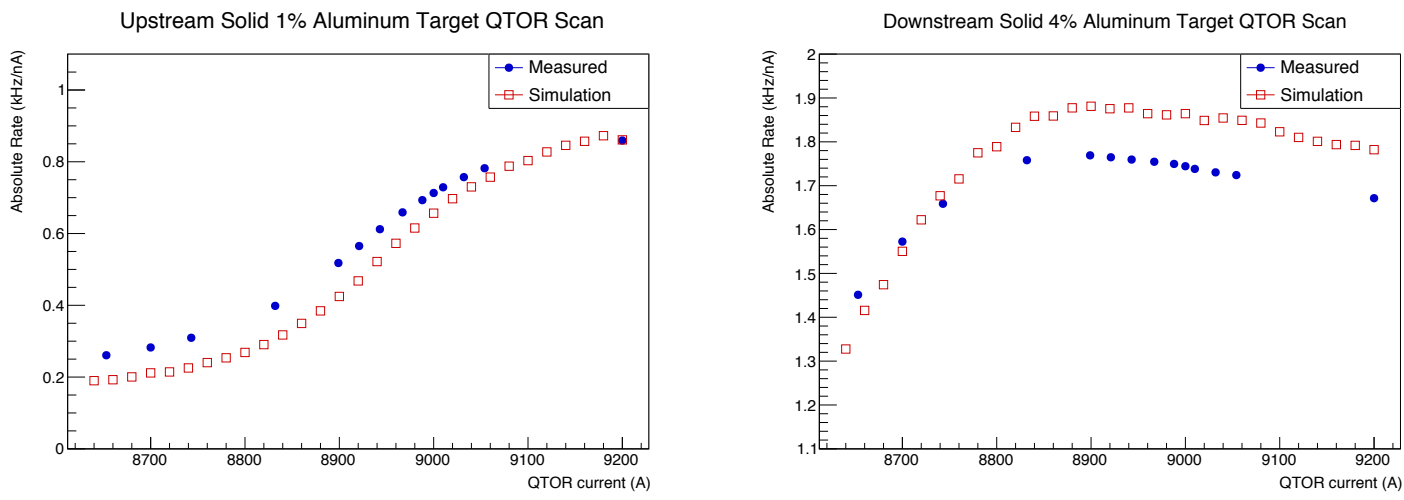

Figure 5.17: Absolute rates during QTOR magnet field scans (measured rates are blue circles, predictions from simulation red squares). The magnetic field is proportional to the magnet current $(\mathrm{A})$.
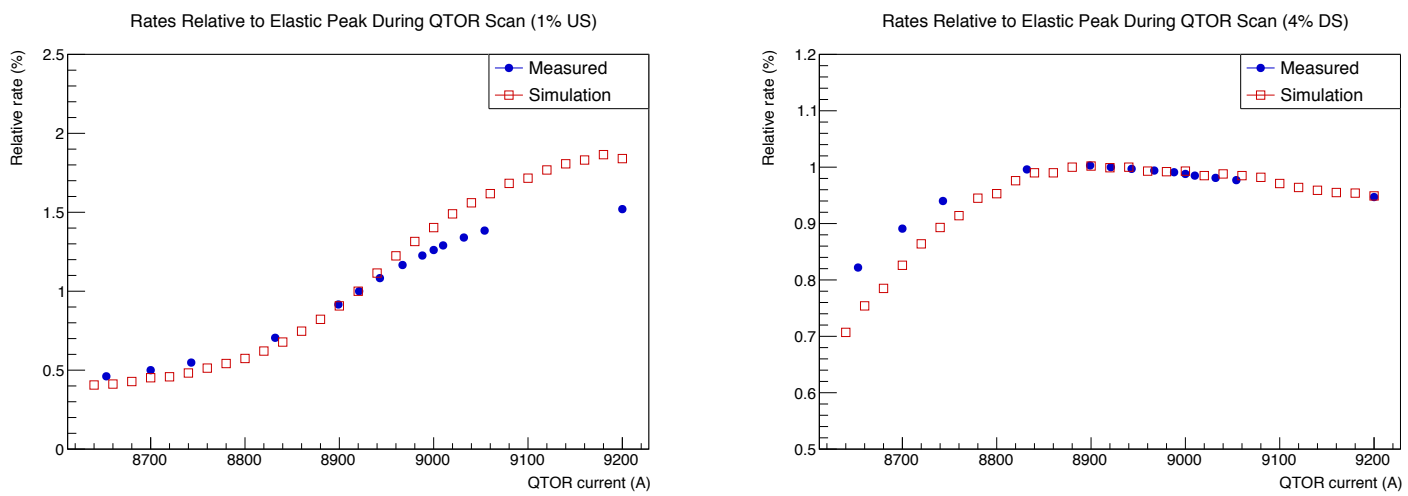

Figure 5.18: Relative rates during QTOR magnet field scans (measured rates are blue circles, predictions from simulation red squares). The rates are normalized to the nominal magnetic setting at $8921 \mathrm{~A}$. 
of QTOR field of 1.5\%. Early simulations suggested that the energy loss from adding $\mathrm{LH}_{2}$ into an empty target is roughly equivalent to an increase of $1 \%$ in the QTOR field. Therefore, to be conservative, a $25 \%$ model uncertainty is assigned for these discrepancies between simulation and data. Since the radiative correction determined in Section 5.4 is approximately $6 \%$, this corresponds to a $1.6 \%$ uncertainty on the final dilution value.

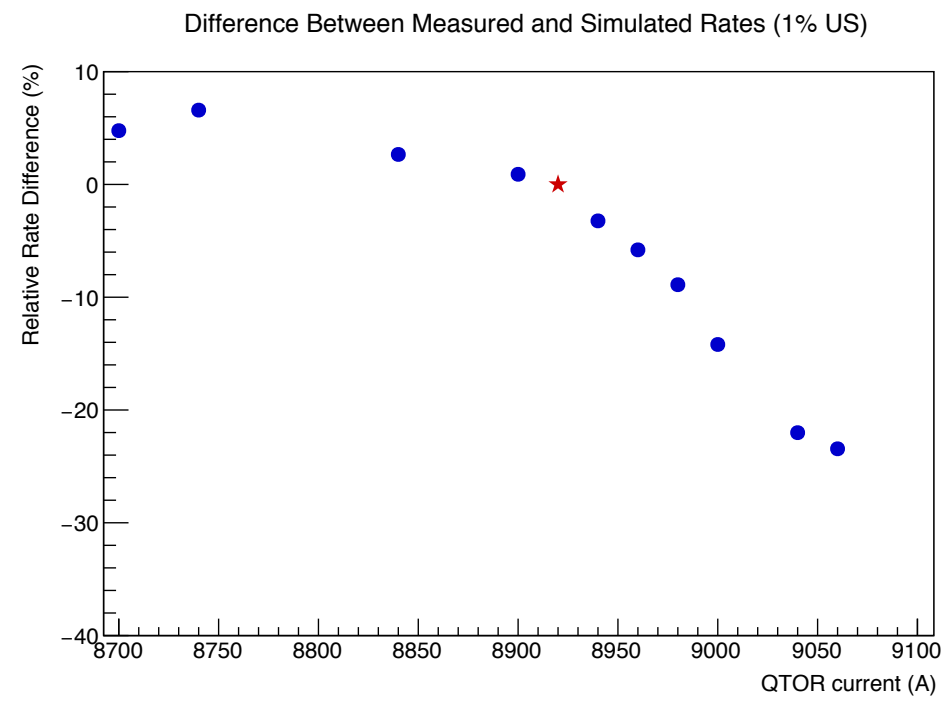

Figure 5.19: Relative upstream 1\% aluminum solid target rate difference between measured rates and predicted rates, in percent, versus magnet current.

\subsection{Dilution final results}

The octant-average of the dilutions, including uncertainties are given in Table 5.6. Figure 5.20 shows the final radiatively corrected dilution factors by octant. The orange box is the deadtime correction model uncertainty, the combined yellow and orange boxes give the total uncertainty from the model correction and BCM uncertainty, while the combined yellow, orange, and green boxes give the total model uncertainty, including the simulation model dependence. The simulation model uncertainty is only applicable for the radiatively corrected points (red squares). The uncertainty of the corrected points is large compared to the raw data from the simulation statistics. The final dilution uncertainty is approximately $2.8 \%$ of itself. 


\begin{tabular}{|l|c|c|c|c|c|c|}
\hline & $\begin{array}{c}f_{\text {average }}\left(\times 10^{-2}\right) \\
\left(\times 10^{-4}\right)\end{array}$ & $\begin{array}{c}\text { stat+sys } \\
\left(\times 10^{-4}\right)\end{array}$ & $\begin{array}{c}\text { BCM } \\
\left(\times 10^{-4}\right)\end{array}$ & $\begin{array}{c}\text { simulation model } \\
\left(\times 10^{-4}\right)\end{array}$ & $\begin{array}{c}\text { total } \\
\left(\times 10^{-4}\right)\end{array}$ \\
\hline Run1 & 2.869 & 2.321 & 4.304 & 4.304 & 4.639 & 7.997 \\
\hline Run2 & 2.851 & 2.310 & 4.286 & 4.285 & 4.616 & 7.959 \\
\hline
\end{tabular}

Table 5.6: The octant-averaged dilution with higher order correction for Runs 1 and 2 , with uncertainties. The (stat+sys) column is the contribution due to statistics and accidental corrections. The deadtime model and BCM uncertainty are both assessed at $1.5 \%$. The simulation model uncertainty remains under active investigation at this time.

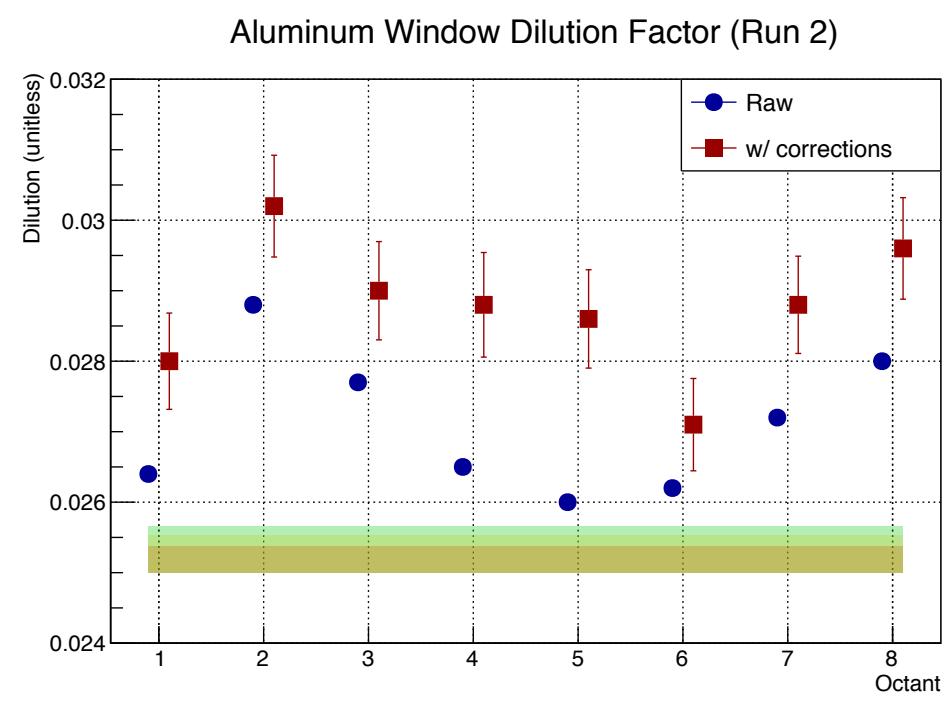

Figure 5.20: Dilution factors including radiative corrections for both Run 2. The uncertainty boxes are for the deadtime model (orange), the quadrature sum of deadtime and BCM uncertainty (yellow), and the total model error, including simulation uncertainties (green). 


\section{Chapter 6}

\section{The Parity-Violating Asymmetry of Aluminum}

HAPTER 3 INTRODUCED the aluminum contribution in $Q_{\text {weak }}$, concentrating on the rate fraction of events from the target walls. Equally important is determining the parity-violating asymmetry from the target's aluminum alloy, which is this chapter's focus. For the $Q_{\text {weak }}$ Run 0 result [48], the parity-violating asymmetry of the aluminum alloy was determined to $15 \%$ of itself; in this chapter the full statistical power of the Run 2 dataset will be utilized, bringing the final uncertainty to below $5 \%$. Aside from being necessary in the analysis of $Q_{\text {weak }}$, it is possible to extract the parity-violating asymmetry of pure aluminum from this dataset as well. A short discussion of the requirements of such an analysis is found in Chapter 7. It is expected this latter measurement will be published within the next year.

\subsection{Aluminum asymmetry overview}

A thick aluminum dummy target, made of the same aluminum alloy as the cryotarget walls, was used periodically throughout Run 2 for data taking. Data were split into several convenient sections, depending on the configuration of the polarized source and injector magnets (Section 3.2 describes these in detail). Run 2 was separated into 5 major periods called Wiens, each labeled by the state of the injector solenoid magnet (as discussed in Section 3.2.2). After several weeks of data taking in one configuration, the solenoid magnet polarity was reversed, thereby reversing the sign of the experimentally produced electrons relative to the laser helicity at the photocathode. Each magnet reversal constituted a new Wien period. There were 11 Wiens in $Q_{\text {weak }}$ : Run 0 was Wien 0, Run 1 consisted of Wiens 1-5, and Run 2 incorporated Wiens 6-10.

Minor divisions, called slugs, were groups of data with a common insertable half- 
wave plate configuation. ${ }^{1}$ Slugs are labeled as half-wave plate in or half-wave plate out; typically they were taken in pairs, with equal amounts of data in each half-wave plate state. Generally slugs contain 4 hours of data each, although some slugs contain 8 hours. The goal was to keep similar amounts of data in each slug, and a similar number of slugs in each Wien, to balance statistical power between slug and Wien pairs.

The smallest division of data was a runlet. Runlets comprised approximately 6 minutes of data. Anywhere from 10-12 runlets combined into roughly hour long runs. Generally beam properties and detector response were investigated at the runlet level for purposes of monitoring data quality. One notable exception was looking at runlets immediately after changing the half-wave plate or Wien states. During these periods, other changes to the polarized source were required to minimize charge asymmetry. These transition runs were often scrutinized at the level of quartets.

In all, Run 2 aluminum data was taken in 4 Wiens and 36 slugs. Each period can be labeled with its number $(6,8,9,10)$, or its state (i.e., "In, flip-left" or "Out, flip-right"). Wien 6 was unique as it was 2-pass beam, causing an extra polarization reversal, identical to a Wien flip. Therefore Wiens 6, 8, and 10 all shared similar injector configurations. No aluminum data were taken during Wien 7.

The aluminum data were divided into three groups based on rigorous data quality criteria. Good runs meet all the data quality requirements, bad runs fail for one or more of these criteria, while suspect runs have one parameter at the limits of the acceptable bounds (e.g., charge asymmetry in transition runs). Generally, suspect runs underwent further analysis with the hope they may be more definitively categorized. For the final analysis only good runs were included. A full description of the data quality requirements are found in Section 6.2.

\subsection{Data quality}

Two primary types of data quality conditions were enforced. The first were automatic cuts based on beam and detector hardware parameters, such as beam current and accelerator cavity stability. For example, if one of the SRF accelerator cavities tripped off, any data collected during its restoration of beam would be cut. These periods of data cuts lasted until the stable beam current was returned. These software cuts are discussed in [122].

Other hardware or device cuts were also implemented outside of software. These

\footnotetext{
${ }^{1}$ This was the insertable half-wave plate positioned before the Pockels cell on the laser table.
} 
primarily include hardware checks; for example there were periods where the QTOR power supply would temporarily fail or shut-off mid-run, and several runs where automated charge feedback systems failed leading to excessively large charge asymmetries (several thousand ppm). Since regression studies require at least 5000 quartets per runlet, runlets with fewer than 5000 quartets were also discarded. The full list of these cut runlets are documented in the $Q_{\text {weak }}$ electronic logbook $[123,124]$, and consisted of approximately 200 runlets. The original raw dataset consisted of approximately 2200 runlets.

After these principal cuts were enacted, a secondary set of standards were developed and implemented, ultimately sorting each runlet into good, suspect, or bad groupings. Initial sorting was based on beam charge asymmetry, or the subtle difference in the amount of beam charge produced between positive and negative helicity states. ${ }^{2}$ Large charge asymmetry affects the data adversely if there is any small non-linear response in the detector chain, and acceptable levels are typically several parts per million.

During data taking, the charge asymmetry was minimized by an active charge feedback mechanism (Section 3.2.1) and careful configuration of the polarized source optical components. After changing the half-wave plate state, the Pockels cell voltage is reconfigured. During these transition periods charge asymmetry is unacceptably large and unstable. Each transition runlet was individually investigated, and labeled as acceptable, unacceptable, or borderline.

Figure 6.1 is an example of an acceptable transition runlet. The charge asymmetry appears statistically distributed around zero with no drifting. Figure 6.2 shows an example of a clearly unacceptable transition run. The charge asymmetry is large, switches sign, and only falls to zero at the end. Figure 6.3 shows the third class, where the average charge asymmetry is relatively small, but shows large drifts. These borderline runs were marked as suspect and removed from this analysis.

Runs with excessively large average charge asymmetry (>30 ppm) were immediately discarded. Figure 6.4 shows the average charge asymmetry of the remaining runlets; the charge asymmetry average is consistent with zero. A Gaussian fit is shown in red. The fit width is $1.1 \mathrm{ppm}$. Runlets within $5 \sigma$ of zero were marked good, while runlets between 5-10 $\sigma$ of zero were marked suspect, to be conservative. All runs outside of the $10 \sigma$ acceptance were individually investigated, but were ultimately discarded due to other data quality problems.

\footnotetext{
${ }^{2}$ This might be from differing amounts of residual linear polarization in the laser light entering the Pockels cell.
} 


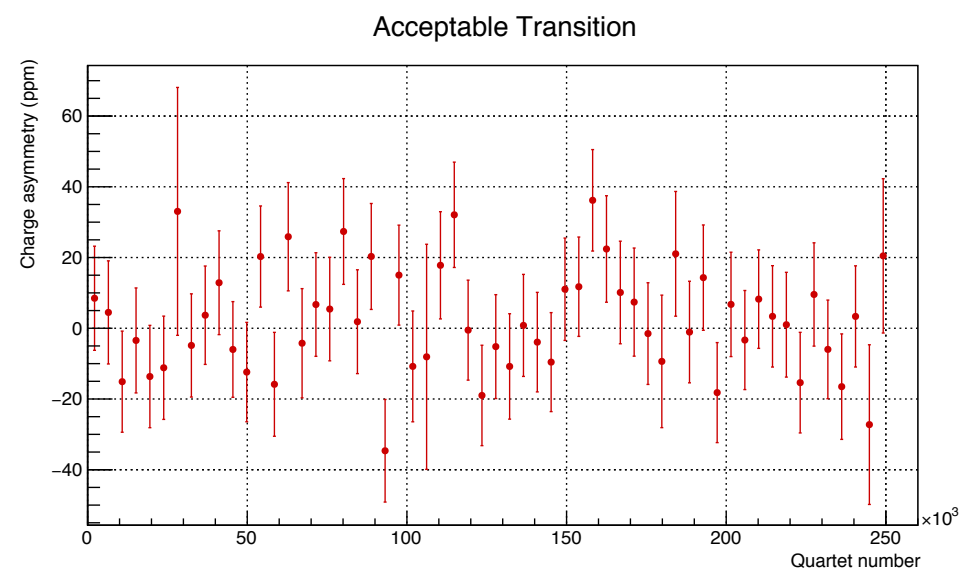

Figure 6.1: Beam charge asymmetry minimization in an acceptable transition run.

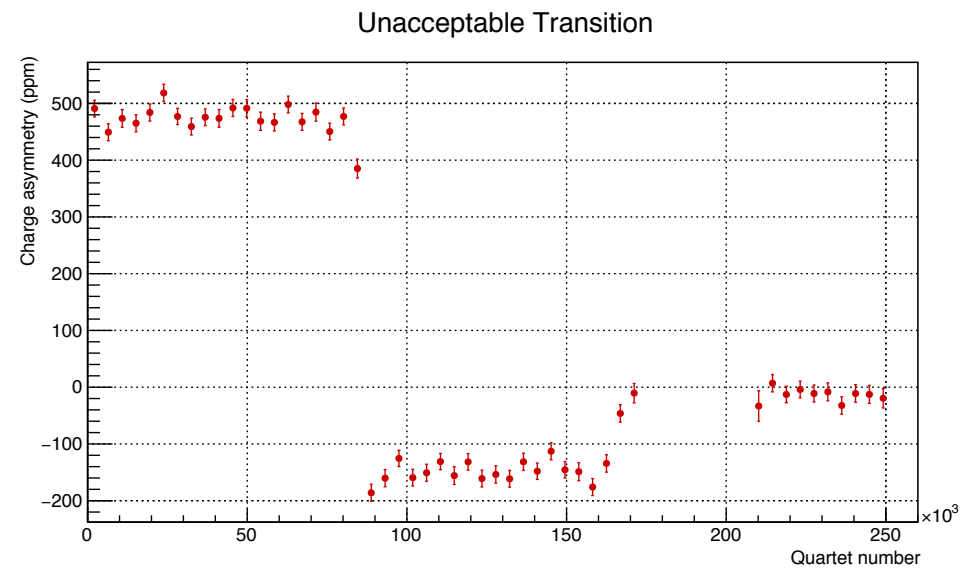

Figure 6.2: Beam charge asymmetry minimization in an unacceptable transition run.

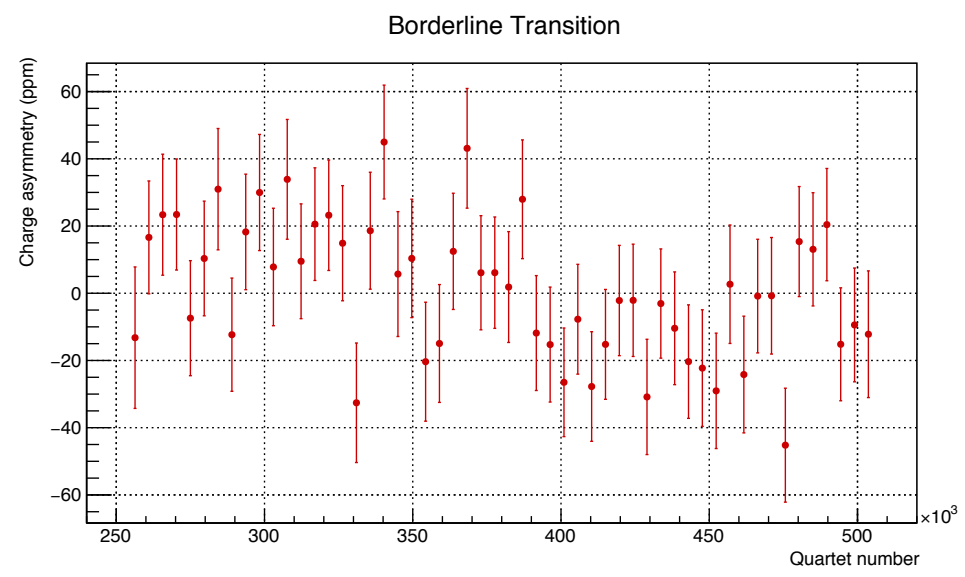

Figure 6.3: Beam charge asymmetry minimization in a borderline transition run. 


\section{Charge Asymmetry for Aluminum Data (ppm)}

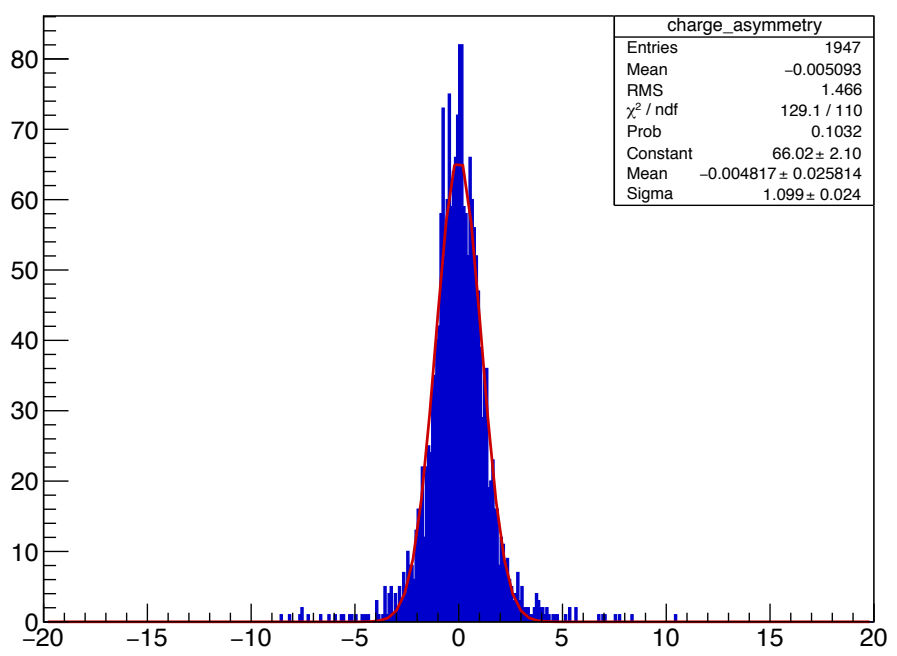

Figure 6.4: Histogram of the charge asymmetry for Run 2 data on the aluminum target for good and suspect runs. The red line is a Gaussian fit which has a reasonable reduced$\chi^{2}$.

After the charge asymmetry cuts, various detector outputs and beam properties were meticulously investigated $[123,125]$. Runlets with significant variations in detector signal, detector output width, or beam properties were flagged for further investigation; runlets were not cut based solely on these deviations.

Two periods early in Wien 8 were particularly noticeable for fluctuations in both beam parameters and detector response. The first was a period of bad transmission in Slug 1038, and early in Slug 1043. Charge asymmetry had been increasing during $\mathrm{LH}_{2}$ production running immediately preceding the aluminum measurements. To compensate, settings of magnets located in the injector were changed, but accidentally resulted in large shifts in the $\mathrm{BCM}$ double difference widths, indicating excessive noise or fluctuations in beam current (see Section 3.3.2). Fluctuations were also seen in the helicity-correlated energy differences and the energy difference widths for this period, although these effects might be uncorrelated.

The second period occurred in Slug 1035 during a series of accelerator tests using the new $100 \mathrm{MeV}$ accelerator SRF cavities. These cavities were developed for the upcoming $12 \mathrm{GeV}$ energy upgrade at JLab, and were periodically used for beam studies throughout Run 2. Figures 6.5 and 6.6 show the BCM double differences and energy difference during Wien 8. The fluctuations are clearly apparent. To be conservative, both of these periods were marked as suspect.

During slugs 1059-1061 (Wien 10) there were read-out problems in BPM 3c12. As 


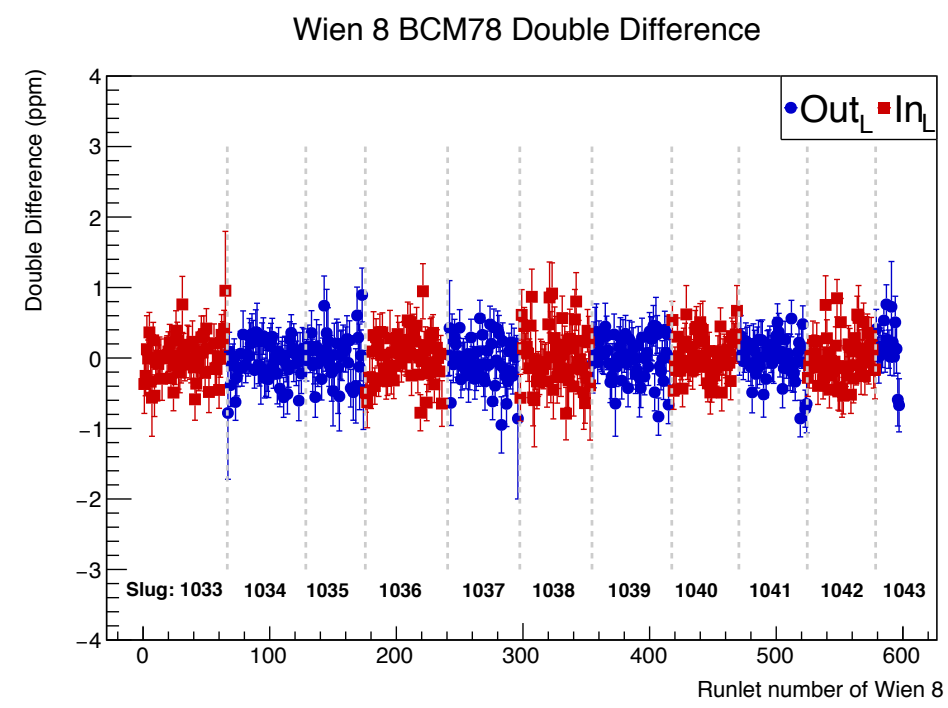

Wien 8 BCM78 Double Difference RMS

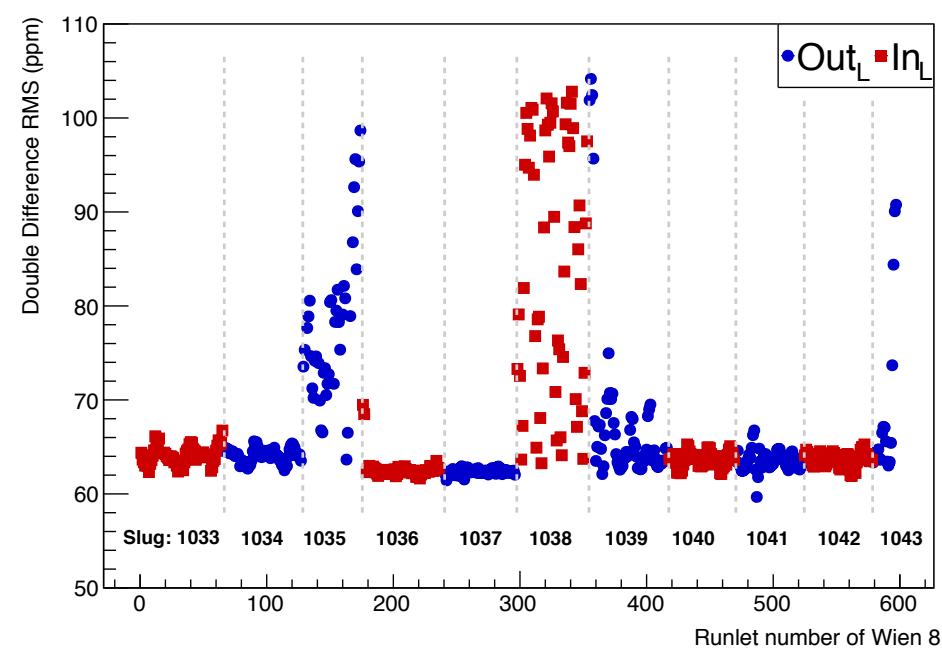

Figure 6.5: BCM78 double differences (top) and their RMS (bottom) by runlet. The slugs are labeled on the plot. All good and suspect data are shown. The variances in Slug 1038 and beginning of Slug 1039 are periods of bad transmission, while those in Slug 1035 are from C100 SRF cavity tests.

discussed in Section 3.3.3, BPM 3c12 functions as an important energy monitor in the arc. Most of the original regression schemes require BCM 3c12, so an additional regression scheme (set13) using BPM 3c11 was developed to facilitate a regression analysis with the full aluminum data set. BPM 3c11 is a reasonable energy monitor, having half the energy sensitivity as BPM 3c12.

The final analysis was conducted using both the good data separately, and a combination of both good and suspect data combined to look for possible systematic effects. The combined good and suspect data set contained 1953 runlets and $1.03 \times 10^{9}$ quartets, while the good-only data set contained 1822 runlets and $9.68 \times 10^{8}$ quartets. 


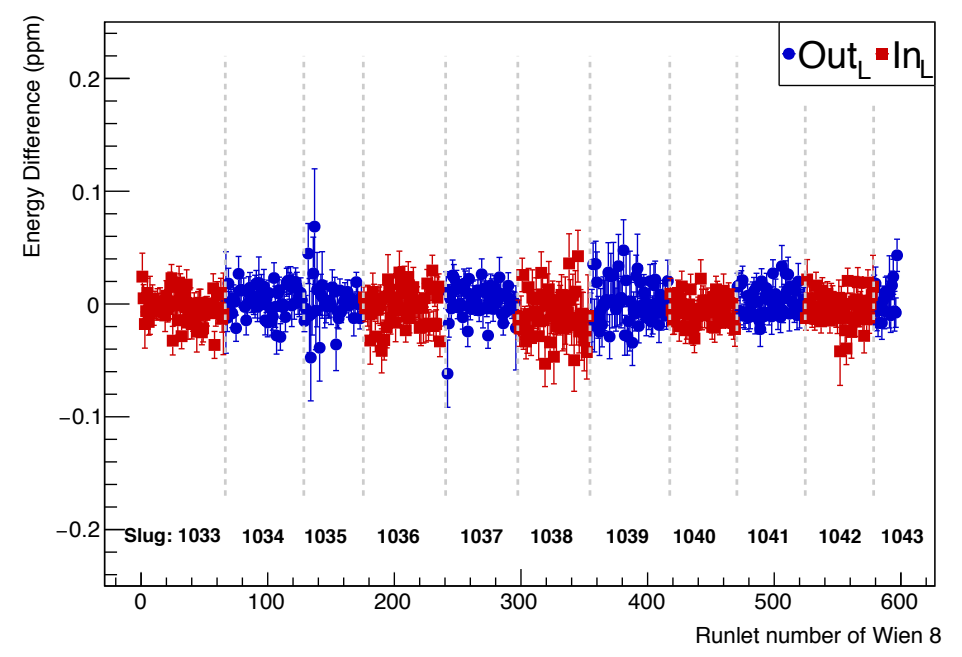

Wien 8 BCM78 Energy Difference RMS

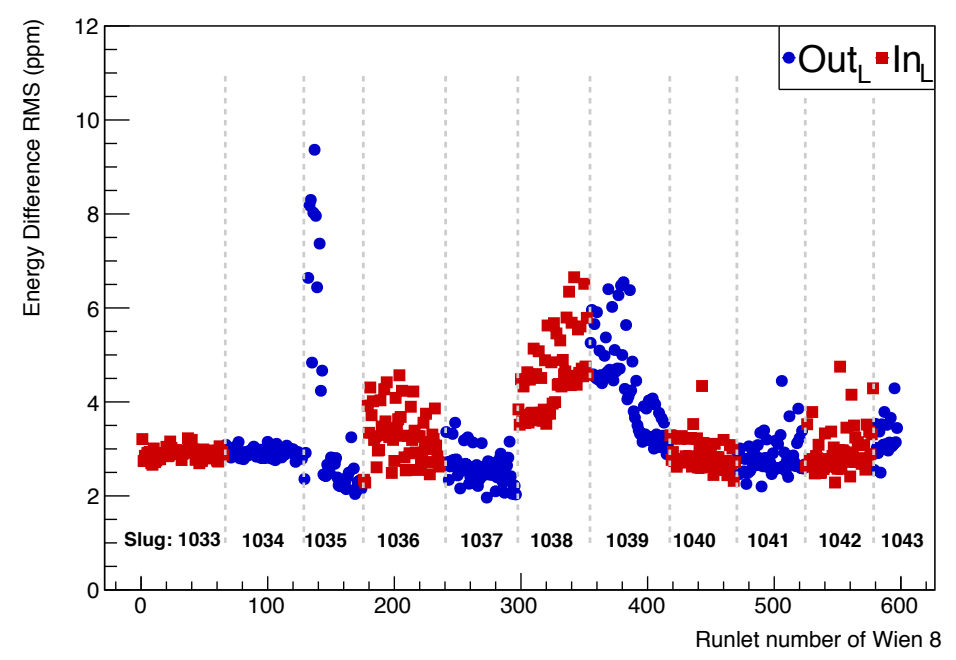

Figure 6.6: Helicity-correlated energy differences (top) and their RMS (bottom) by runlet. The slugs are labeled on the plot. All good and suspect data are shown. The variances in Slug 1038 and beginning of Slug 1039 are periods of bad transmission, while those in Slug 1035 are from C100 SRF cavity tests.

Recently, an interesting effect was discovered. A best fit line to a constant was determined for each individual main detector asymmetry over all of Run 2 (the asymmetries were sign-corrected). The probability of observing a $\chi^{2}$ at least as extreme by chance is shown in Figure 6.7. The probabilities are shown for all eight detectors, including all 4 Wien periods (blue circles) and all but Wien 10 (red squares). Ideally, the fit probability should be around 0.50. Note that for main detectors 6,7 , and 8 the fit probabilities are quite unlikely (the probabilities are $\sim 0.001$ ). The likelihood of getting a $\chi^{2}$ as extreme by chance is approximately 1 in a million.

This recent finding has initiated further investigation, but these have born little 
Fit Probability to Sign-Corrected Asymmetry

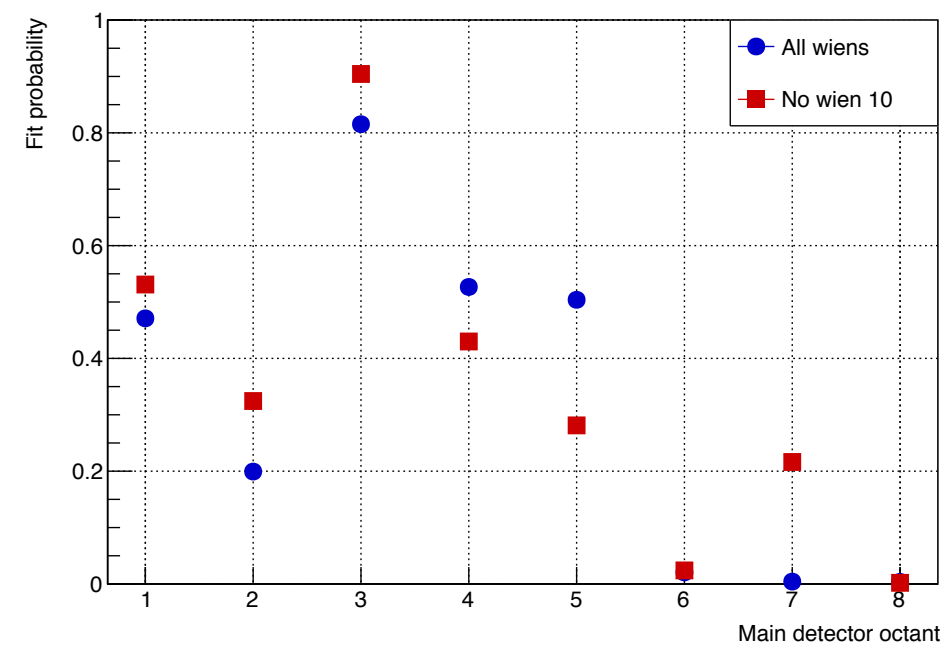

Figure 6.7: The sign-corrected main detector asymmetries were fit over all of Run 2 . Plotted is the probability of observing a $\chi^{2}$ at least as extreme as observed by chance, with (blue circles) and without (red squares) Wien 10.

fruit. The most notable finding was that even after beamline background corrections, large residual correlations to the beam parameters $Y$ and $Y$-angle remain during Wien 10. Since removal of Wien 10 improves the fit in octant 7 and also eliminates the residual correlations, some advocate discarding it ${ }^{3}$. However, one should be cautious about choosing a data set based solely on the measured main detector asymmetry alone. So, with no cogent explanation demanding its dismissal, Wien 10 remains labeled good and is included in the analysis.

\subsection{Asymmetry analysis}

The measured parity-violating asymmetry of the aluminum target alloy is presented in this section. All analysis includes only runs labeled good, as discussed in Section 6.2, unless otherwise specified. Data will routinely be separated into positive and negative averages; these are periods where the expected asymmetry is positive or negative, respectively, due to the half-wave plate and Wien configurations.

One useful metric for examining possible problems due to helicity-correlated systematics involves constructing the NULL asymmetry. The NULL asymmetry is the sum of the average negative and positive asymmetries; any significant non-zero result indicates improper cancellation of false asymmetries which must be investigated and removed. The physics asymmetry is calculated by taking the difference of the weighted-average

\footnotetext{
${ }^{3}$ Discarding Wien 10 would increase the final statistical uncertainty of this analysis by less than 10 ppb.
} 
positive and negative asymmetries. These averages are usually analyzed at different times scales (Wiens, slugs, and runlets) to better understand any time dependencies.

Corrections must then be made for various backgrounds and polarization. The measured asymmetry, $A_{\text {meas }}$, is related to the true asymmetry, $A_{A l}$, by

$$
A_{\text {meas }}=P\left[\left(1-\sum_{i} f_{b k g d}^{i}\right) A_{A l}+\sum_{i} f_{b k g d}^{i} A_{b k g d}^{i}\right],
$$

where $f_{b k g d}^{i}$ and $A_{b k g d}^{i}$ are the background dilutions and asymmetries, respectively. For the aluminum contribution in $Q_{\text {weak }}$, the only relevant background sources are the beamline and QTOR transport backgrounds. Extracting the pure aluminum parity-violating asymmetry will require correcting for other background sources such as contaminants, detector acceptance, and radiative effects.

Unlike other corrections, the exact origins of the neutral beamline backgrounds are not well established, although the favored explanation is helicity-correlated beam components interacting with either the beamline or the tungsten plug. These components could be helicity-correlated beam halo changes or possibly position shifts [126]. The background asymmetry measured by the main detectors is tightly correlated with the upstream luminosity monitor asymmetries. These correlations are extracted over the course of Run 2, and applied at the slug level (the size of the correction is time dependent). This dilution is time independent, and is measured during studies where large tungsten shutters are installed in the collimators, blocking two octants. This analysis is the subject of [127].

The QTOR transport backgrounds are primarily neutral backgrounds passing through the collimators. Processes include neutral particles from scattering events off the collimator slit and shielding wall, and are measured in dedicated runs during low-current tracking mode measurements, including runs using the tungsten shutters. The dilution is measured to be $(0.5 \pm 0.1) \%$ [122], but its asymmetry is simulated. The asymmetry is assumed to be from the parent process (elastic or quasielastic scattering, for example). To be conservative, this asymmetry is assumed the full value of the elastic $e \cdot A l$ asymmetry, with $100 \%$ uncertainty.

It is expected that the elastic and quasielastic asymmetries for pure aluminum can be extracted from the $Q_{\text {weak }}$ data, and this extraction will require more in-depth analysis to account for radiative effects and acceptance corrections, much like the full $Q_{\text {weak }} \mathrm{LH}_{2}$ result. These are briefly discussed in Section 7.3. 

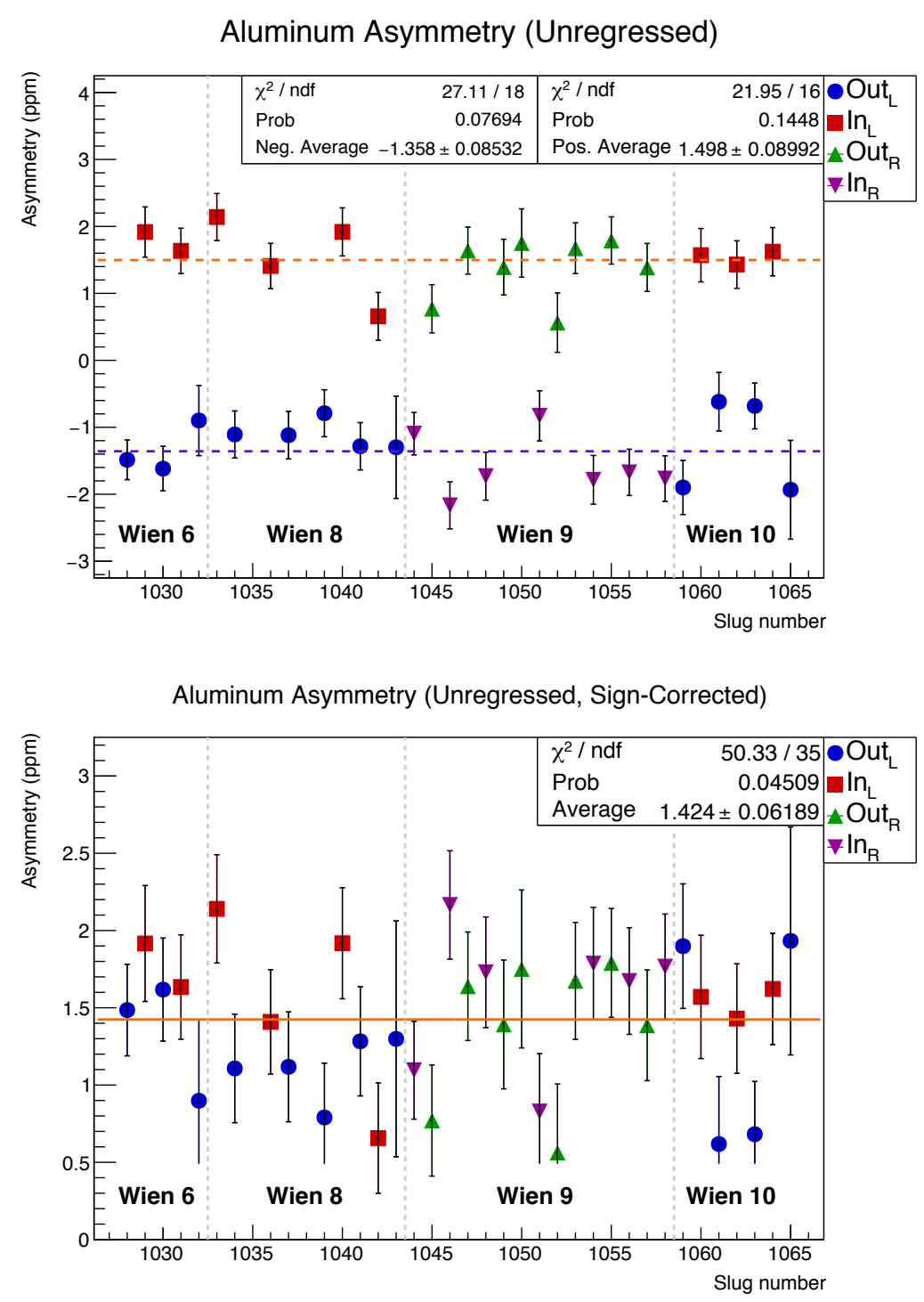

Figure 6.8: (top) Unregressed asymmetry by slug; (bottom) including sign correction.

\subsubsection{Raw asymmetry and background corrections}

The unregressed measured asymmetry is summarized in Figures 6.8 and 6.9, and Table 6.1. These data include neither beamline background nor polarization corrections. The fits are to a constant, and although none of the fits are unrealistic, the negative state fits are relatively poor as judged by their reduced $\chi^{2}$ and fit probabilities. In particular, Wien 8 has a reasonably large NULL asymmetry, $1.8 \sigma$ from zero. The other Wiens have NULLs consistent within $1 \sigma$ of zero. The statistical precision is $61.9 \mathrm{ppb}$ including only the good data. This is approximately a $4.4 \%$ precision measurement. Discarding Wien 10 would increase the statistical uncertainty to approximately $64.6 \mathrm{ppb}$, a 2.7 ppb increase.

For the aluminum analysis, the regression scheme used was set13, which uses BPM3c11 

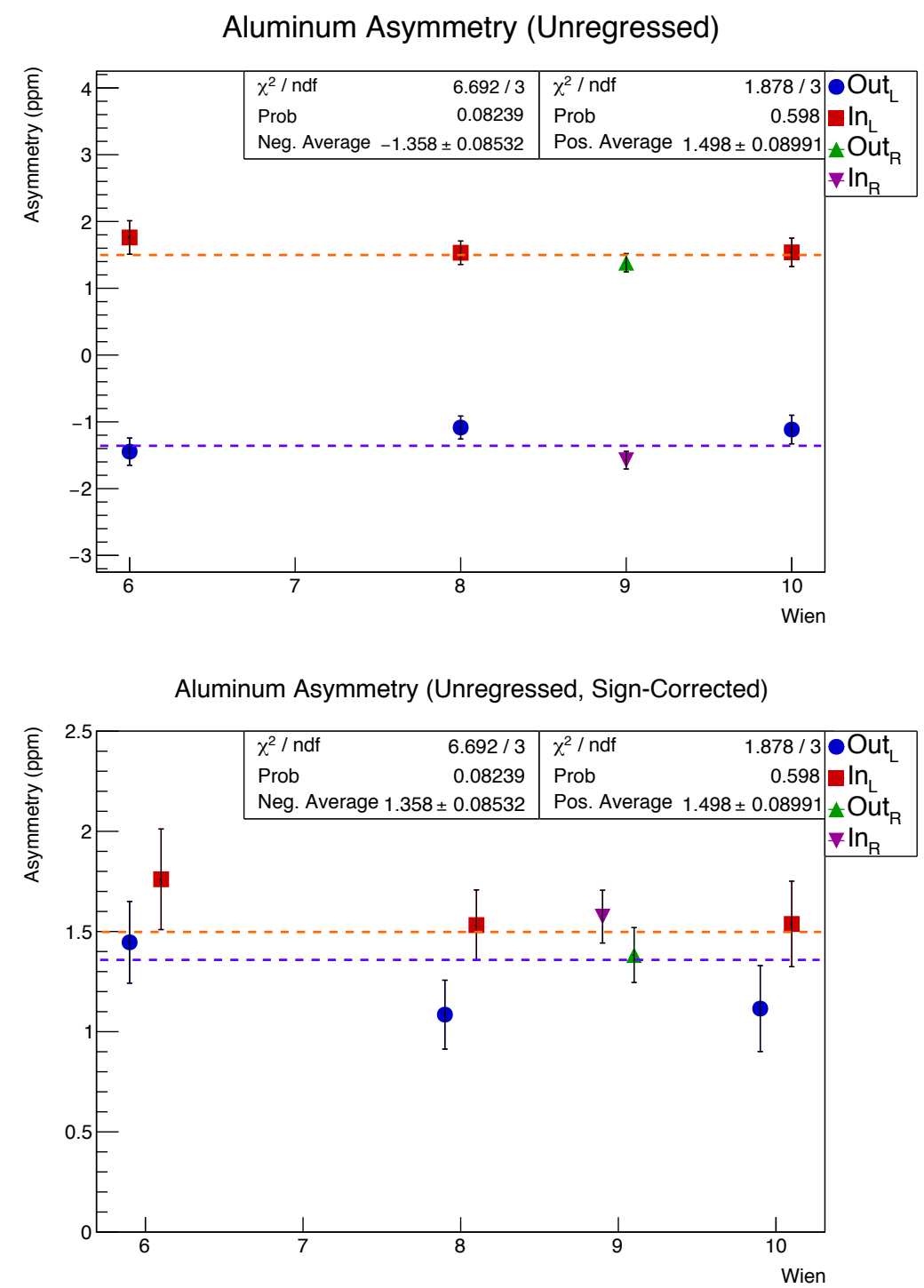

Figure 6.9: (top) Unregressed asymmetry by Wien; (bottom) including sign correction.

\begin{tabular}{|l|c|c|c|c|}
\hline State & Asym $(\mathrm{ppm})$ & Error $(\mathrm{ppm})$ & $\chi^{2} /$ ndf & Prob \\
\hline NEG & -1.3583 & 0.0853 & 1.5 & 0.076 \\
\hline POS & 1.4978 & 0.0899 & 1.37 & 0.145 \\
\hline NULL & 0.0697 & 0.0620 & 0.18 & 0.912 \\
\hline PHYS & 1.4244 & 0.0619 & 1.44 & 0.045 \\
\hline
\end{tabular}

Table 6.1: Summary of the extracted aluminum asymmetry by slug before background and polarization corrections. NEG and POS refer to the averaged negative and positive state asymmetries, while NULL and PHYS give the average difference and weightedaverage sum of NEG and POS, respectively. 
Aluminum Asymmetry (Regressed, set13)

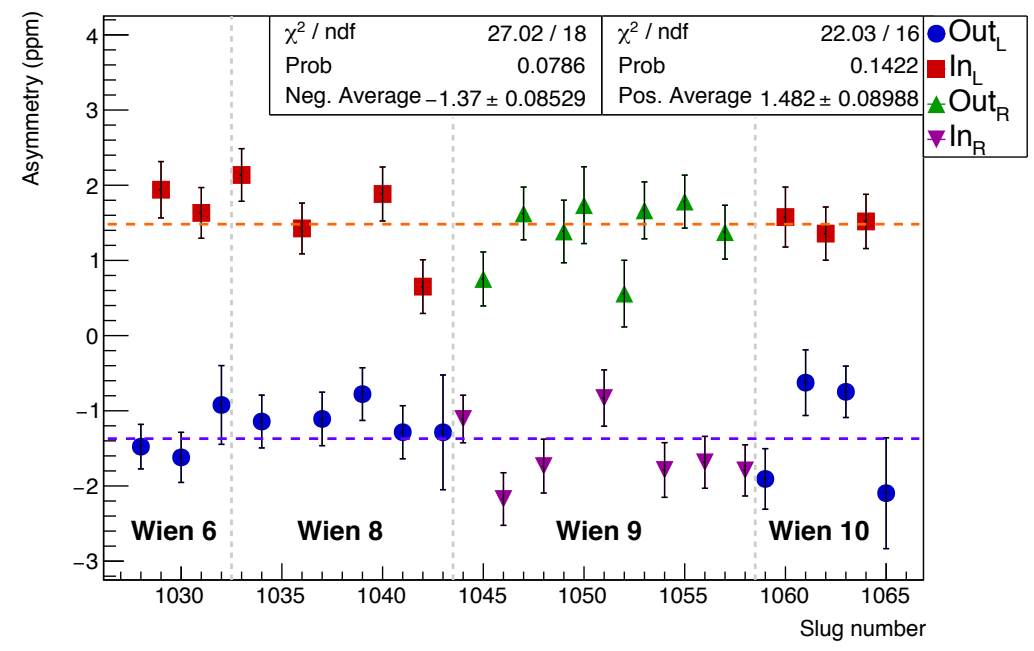

Aluminum Asymmetry (Regressed Set13, Sign-Corrected)

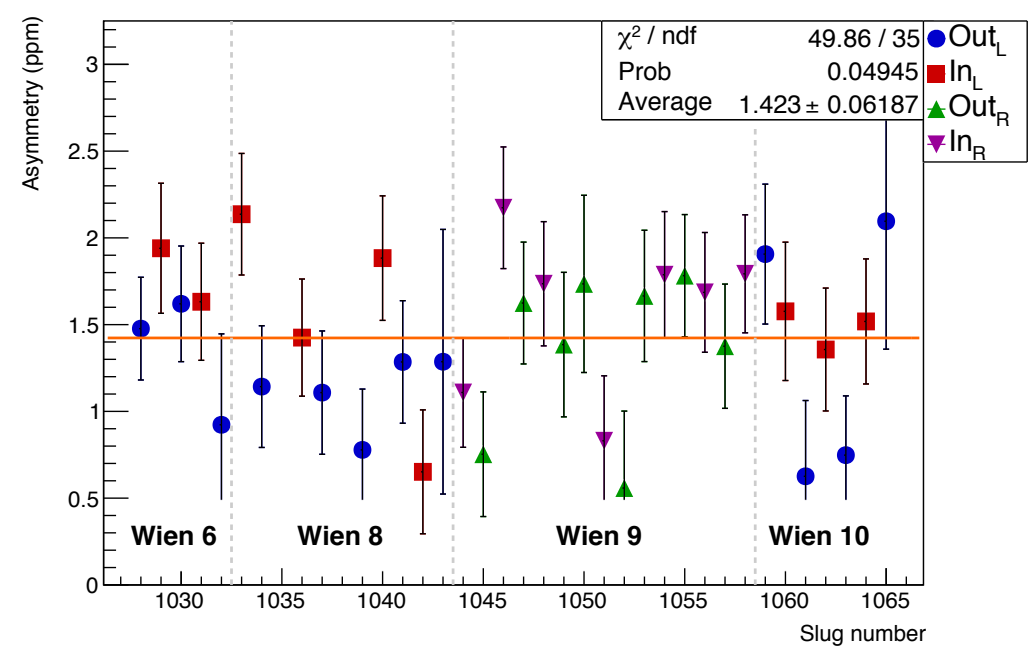

Figure 6.10: (top) Slug asymmetries, regressed using regression scheme set13; (bottom) including sign correction.

as an energy monitor. Figure 6.10 shows the regressed aluminum asymmetry for each slug in Run 2. An analysis of the effects of the other regression schemes was conducted, and is summarized at the end of this section in Table 6.6. This shows that variation in calculated asymmetry after applying regression is on the order of several parts-per-billion. The various regression schemes were discussed in Section 3.3.4.

The effect of beamline background correction is largely negligible, except for a shift of $\sim 50 \mathrm{ppb}$ in Wien 6 IN (see Figure 6.11). This large shift originates in slug 1029 . The main detector weighted-averaged asymmetry for slug 1029 is $1.94 \pm 0.37$ before the correction and $1.82 \pm 0.37 \mathrm{ppm}$ afterwards, a correction of approximately $100 \mathrm{ppb}$. After investigation no cause or explanation has been found, and although large, this is within the slug's statistical uncertainty. 
Beamline Background Correction Sizes

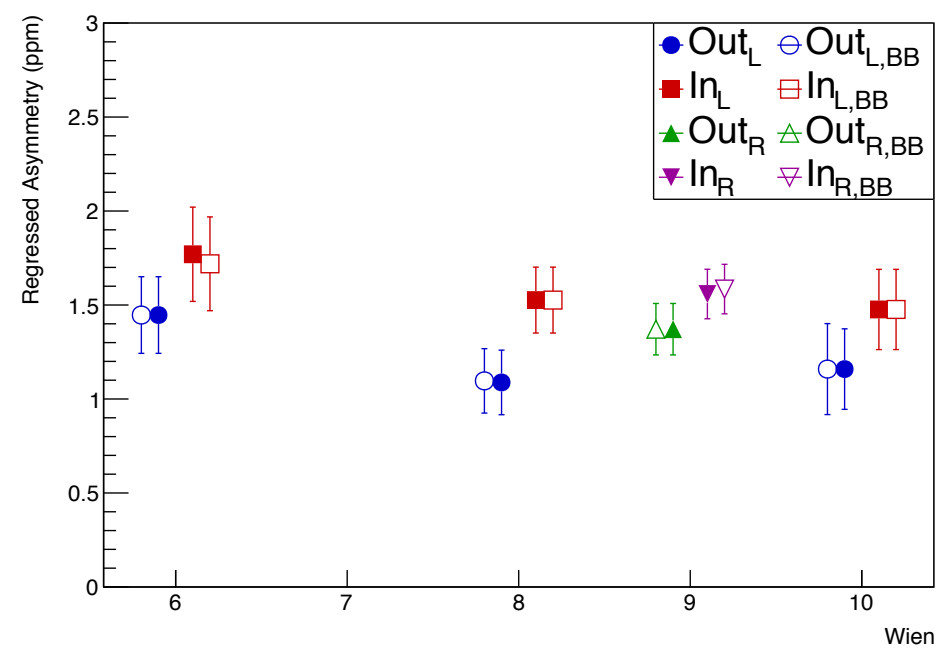

Figure 6.11: Beamline background correction sizes by Wien and half-wave plate state. Corrected data are open markers, while un-corrected data are solid markers. Polarization has not been taken into account.

\begin{tabular}{|l|c|}
\hline Slug Range & Polarization(\%) \\
\hline $1033-1043$ & 88.7 \\
\hline $1044-1047$ & 89.5 \\
\hline $1048-1052$ & 89.7 \\
\hline $1053-1058$ & 87.7 \\
\hline $1059-1065$ & 87.0 \\
\hline \multicolumn{2}{|c|}{ Systematic uncertainty: $0.62 \%$} \\
\hline
\end{tabular}

Table 6.2: Slug averaged polarization values.

Polarization corrections are made on a slug basis. These numbers used combined results from both Møller and Compton polarimeters. The corrections by slug range are given in Table 6.2. The total systematic error for the combined polarization analysis is $0.62 \%$, and this is added in quadrature only to the final calculated value to avoid treating this as a statistical uncertainty.

Figures 6.12 and 6.13 show the slug and Wien-averaged asymmetries after both the beamline background and polarization corrections. Table 6.3 summarizes the overall NULL and extracted physics asymmetries. The fits for the negative states remain rather poor, although still statistically acceptable.

To extract the true aluminum alloy asymmetry, corrections for the neutral QTOR transport channel must now be accounted for. As discussed previously, the associated dilution factor is taken to be $f_{\text {qtor }}=(0.08 \pm 0.08) \%$. Combined with the assumed asymmetry of $1.606 \mathrm{ppm}$, the total correction is $1.28 \pm 1.28 \mathrm{ppb}$. After scaling by the total dilution factor $\left(f_{\text {tot }}=f_{\text {qtor }}+f_{\text {beamline }}=0.77 \%\right)$, the final measured aluminum 

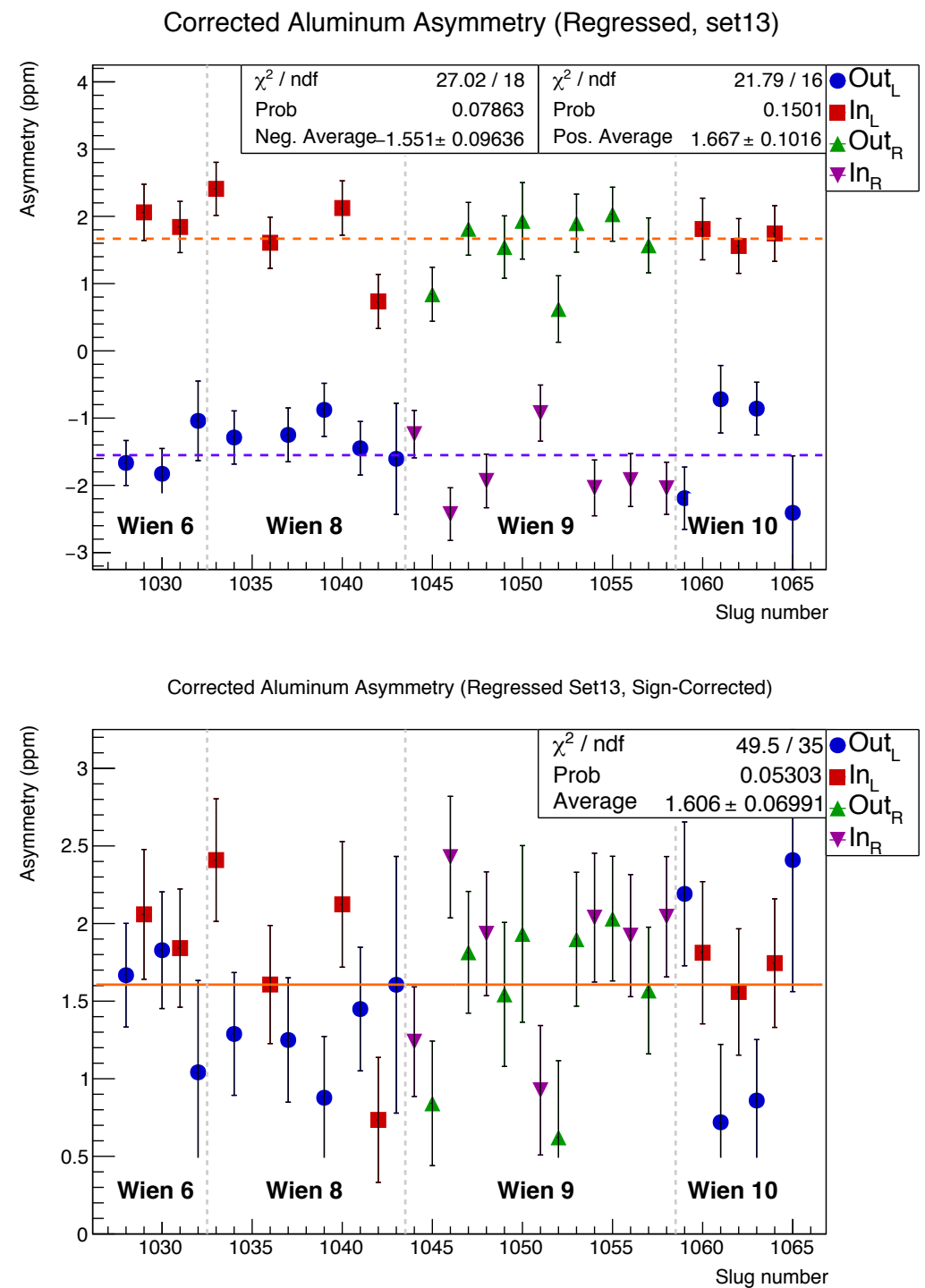

Figure 6.12: (top) Regressed asymmetry by slug after corrections; (bottom) including sign correction.

\begin{tabular}{|l|c|c|c|c|}
\hline State & Asym $(\mathrm{ppm})$ & Error $(\mathrm{ppm})$ & $\chi^{2} / \mathrm{ndf}$ & Prob \\
\hline NEG & -1.551 & 0.0963 & 1.50 & 0.079 \\
\hline POS & 1.667 & 0.1016 & 1.36 & 0.150 \\
\hline NULL & 0.0581 & 0.0700 & 0.51 & 0.676 \\
\hline PHYS & 1.606 & 0.0699 & 1.41 & 0.053 \\
\hline
\end{tabular}

Table 6.3: Summary of the corrected aluminum asymmetries by slug. 
Corrected Aluminum Asymmetry (Regressed, set13)
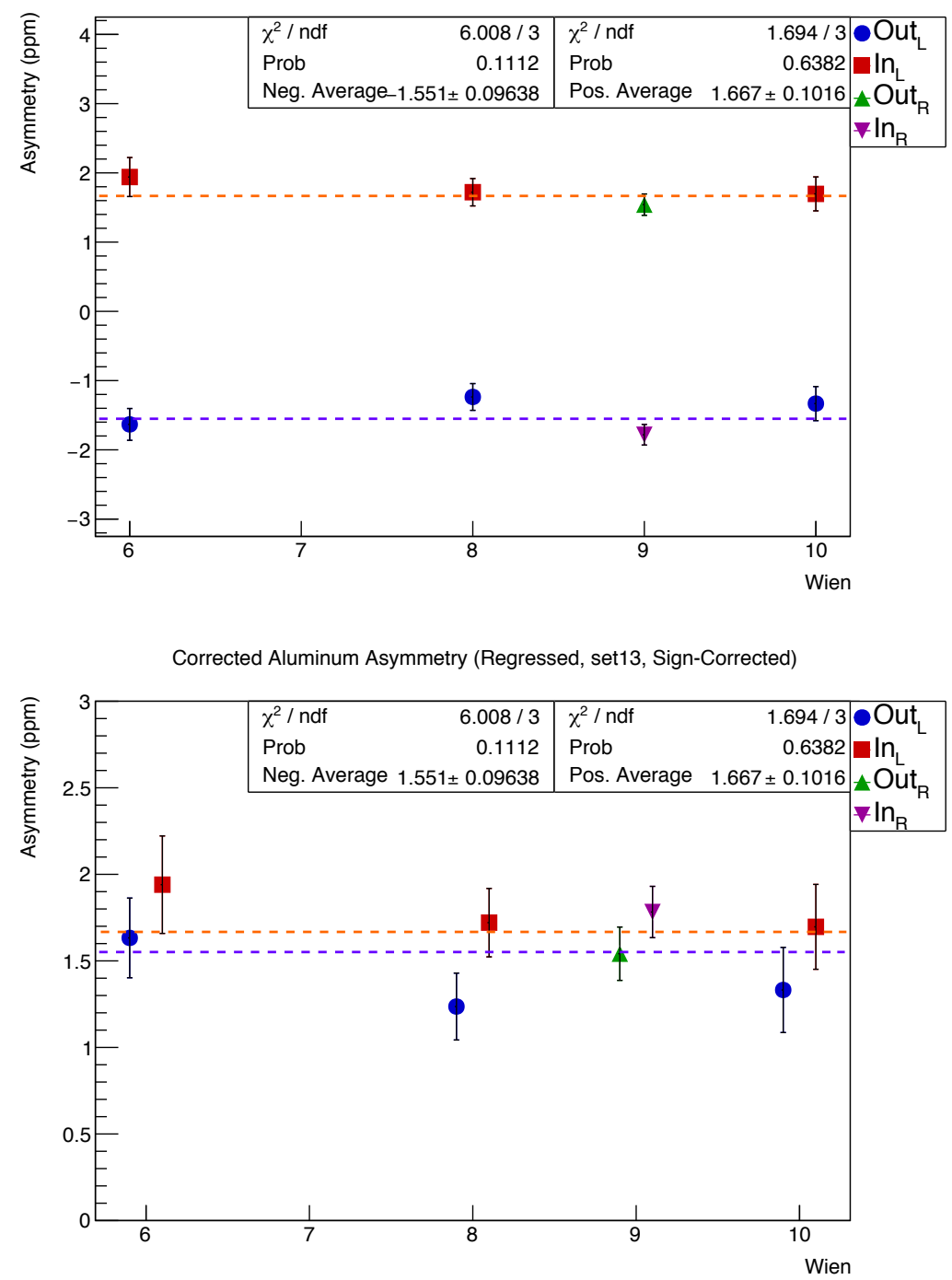

Figure 6.13: (top) Regressed asymmetry by Wien after corrections; (bottom) including sign correction. 


\begin{tabular}{|l|c|}
\hline \multicolumn{2}{|c|}{$\begin{array}{c}\text { vverage Helicity Correlated } \\
\text { Beam Parameters }\end{array}$} \\
\hline X position (nm) & 2.28 \\
\hline Y position (nm) & -5.26 \\
\hline X angle (nrad) & 0.0677 \\
\hline Y angle (nrad) & -0.159 \\
\hline Energy (ppb) & -0.330 \\
\hline
\end{tabular}

Table 6.4: Run 2 average helicitycorrelated beam parameters during aluminum running.

\begin{tabular}{|l|c|}
\hline \multicolumn{2}{|l|}{ Average Main Detector Sensitivities } \\
\hline X position $(\mathrm{ppm} / \mathrm{mm})$ & 27.0 \\
\hline Y position $(\mathrm{ppm} / \mathrm{mm})$ & 25.3 \\
\hline $\mathrm{X}$ angle $(\mathrm{ppm} / \mu \mathrm{rad})$ & -0.393 \\
\hline Y angle $(\mathrm{ppm} / \mu \mathrm{rad})$ & 0.137 \\
\hline Energy $(\mathrm{ppm} / \mathrm{ppm})$ & 0.400 \\
\hline Charge $(\%)$ & 1.10 \\
\hline
\end{tabular}

Table 6.5: Average main detector sensitivities to helicity-correlated position, angle, energy, and charge differences.

asymmetry is $A_{\text {meas }}=1.617 \pm 0.0704$ (statistical).

The systematic uncertainty currently has four main components:

- Polarization

- Detector non-linearity

- Regression scheme dependence

- Effect of including suspect runs

The polarization contribution is trivial: since the polarization uncertainty is $0.62 \%$, its contribution to the aluminum asymmetry is simply $10.0 \mathrm{ppb}$.

The main detectors are known to be non-linear at the $(0.20 \pm 0.20) \%$ level [128]. Being a property of the detector, any measured asymmetry will need to be corrected. The full formalism has not been adequately developed by the collaboration at this time. For this reason, an uncertainty of $3.2 \mathrm{ppb}$ ( $100 \%$ of the effect size) is attributed.

The detector non-linearity can also contribute through the BCMs. In general, the main detector is sensitive to helicity-correlated beam parameters. These sensitivities are used during regression. Helicity-correlated differences in charge, however, might contribute false asymmetries due to the main detector non-linearity as well. Tables 6.4 and 6.5 give the average helicity-correlated parameters and main detector sensitivies during aluminum running, for all parameters. Recall from Figure 6.4 the average charge asymmetry for Run 2 is $\approx 2 \mathrm{ppb}$. The possible effect from detector non-linearity from the $\mathrm{BCM}$ is the product of the average charge asymmetry with the main detector sensitivity to charge: $2 \mathrm{ppb} \times(1.10 \%)$, which is negligible.

The regression correction uncertainty was studied by looking at the effect of using various regression schemes. Table 6.6 summarizes the results of the beamline background corrected asymmetries using various regression schemes. Since the majority of 


\begin{tabular}{|l|c|c|c|c|}
\hline Regression set & Avg $(\mathrm{ppm})$ & Error $(\mathrm{ppm})$ & $\chi^{2}$ & $\operatorname{prob}(\%)$ \\
\hline raw & 1.4226 & 0.0641 & 1.393 & 0.0690 \\
\hline on & 1.4208 & 0.064 & 1.380 & 0.0746 \\
\hline $5+1^{*}$ & 1.4239 & 0.0641 & 1.378 & 0.0755 \\
\hline set3 $^{*}$ & 1.4239 & 0.0641 & 1.378 & 0.0755 \\
\hline set4 & 1.4208 & 0.064 & 1.380 & 0.0744 \\
\hline set7 & 1.4201 & 0.064 & 1.377 & 0.0758 \\
\hline set8 & 1.4250 & 0.0646 & 1.406 & 0.0637 \\
\hline set10* & 1.4222 & 0.0641 & 1.380 & 0.0747 \\
\hline set11 & 1.4208 & 0.064 & 1.380 & 0.0746 \\
\hline set13 & 1.4221 & 0.064 & 1.381 & 0.0743 \\
\hline
\end{tabular}

Table 6.6: Effect of various regression schemes on the average, beamline background corrected aluminum asymmetry. "prob" is the probability of the best-fit reduced- $\chi^{2}$ being at least that extreme by chance. Regression sets with an asterisk $\left(^{*}\right)$ indicate they include regression against charge asymmetry.

the regression schemes require BPM 3c12, the slugs where this device failed were neglected in this study (slugs 1059-1061). This allows for the same dataset to be compared for the difference regression schemes. An additional uncertainty of $3 \mathrm{ppb}$ is included for regression scheme dependence; this is slightly more than the largest mean asymmetry shift (2.8 ppb) between sets from the unregressed value.

When including suspect runs into the analysis, the average asymmetry shifts 0.8 ppb. An additional uncertainty of $1 \mathrm{ppb}$ will be added for this cut dependence uncertainty.

Therefore, the final aluminum asymmetry, including all necessary corrections, is $1.6174 \pm 0.0704$ (stat.) \pm 0.011 (sys.) ppm.

\subsubsection{Extracting the $Q_{\text {weak }}$ correction}

Relating the measured asymmetry of the downstream solid $4 \%$ target to the asymmetry of the actual windows requires slight corrections due to the different window locations and the radiative effect of $\mathrm{LH}_{2}$. These slight kinematic adjustments are similar to those discussed in Section 5.4. Like those higher-order corrections, these kinematic adjustments are also determined through GEANT4 simulations.

The elastic cross section and asymmetry of electron-aluminum scattering were discussed in Section 2.2 and 2.3, but elastic scattering only accounts for approximately $80 \%$ of the total window rate. The other $20 \%$ consists of quasielastic and inelastic scattering, which are possible because of $Q_{\text {weak }}$ 's open acceptance and modest energy resolution (scattered electrons with $100 \mathrm{MeV}$ of energy loss may reach the detector 
focal plane). Reasonable cross-section calculations exist for many of these processes, however wherever possible empirical fits were used. The parity-violating asymmetries of these non-elastic processes are not well known, but various models have been developed to approximate these. Fortunately, many of these processes have small rate fractions ( $\sim 5 \%$ or less), so even if their asymmetry is not well known, their total systematic uncertainty contribution is small. This is because the contribution to the total uncertainty is proportional to each process's fractional rate contribution (for example, see [47]).

The quasielastic (QE) cross section was implemented in GEANT4 using "superscaling" formalism with electron-proton data [129, 130]. As a first-order estimate of the asymmetry, the quasi-static approximation is used, where one assumes that the asymmetry is identical to scattering from a free proton or neutron. The authors of [131] studied this model, but neglected final-state interactions. This naive estimate predicts a cross-section weighted asymmetry of $-0.24 \mathrm{ppm}$. Comparing these results to calculations including final state interactions on deuterium suggest this simple model is approximately one-third of the correct value [132]. The final quasielastic asymmetry is calculated as $-0.9 \mathrm{ppm}$, with $100 \%$ uncertainty to be conservative.

Inelastic processes contribute primarily through three channels. It is immediately emphasized that although clear, precise definitions of these channels exist, inelastic processes are defined differently between high and low energy researchers, often leading to confusion in the literature. Medium and high energy groups typically classify inelastic processes as those exciting individual nucleons (i.e., $\pi$ production), while low energy groups classify whole nucleus excitations as inelastic. Typically the meaning is clear through context; however, the difficulty is that both $\pi$ production and nuclear excited states contribute to the asymmetry, and furthermore, are kinematically indistinguishable due to the large $Q_{\text {weak }}$ acceptance.

The first contributing inelastic process is quasi-static resonance production; that is, $\pi$ production through decay of excited nucleon states (e.g., $N \rightarrow \Delta \rightarrow \pi$ ). These processes are heavily suppressed in $Q_{\text {weak }}$, because electrons must transfer significant energy to the proton for pion production $(\approx 130 \mathrm{MeV})$, making it difficult to be kinematically accepted into the detector. This particular channel is designated simply as inelastic scattering here. This inelastic contribution is, like the quasielastic case, also modeled by $[129,130]$. The asymmetry is assumed to be identical to the elastic asymmetry with a $100 \%$ uncertainty.

Low energy nuclear excitations typically involve much smaller energy transfer (on 
the order of several $\mathrm{MeV}$ ), and therefore are not part of the radiative tail. These interactions excite the whole nucleus, where one or more nucleons are excited into a higher orbital. Additionally, these may involve rotation or vibration of the entire nucleus. Descriptions of these states typically abandon the harmonic oscillator assumption seen in the elastic cross-section (Section 2.2) in favour of shell-model calculations. These are defined here as single particle excited states (SPS) or shell model states.

Lastly, there are additional contributions from collective resonances, where the proton and neutron distributions move synchronously. These require energy transfers in the 10's of MeV, and include the well-known Giant Dipole Resonance, (GDR) which is an isovector excitation, meaning the proton and neutron distributions oscillate out of phase.

Generally, these low energy inelastic states are described by the isospin of their excitation [47, 133], such that:

$$
A \approx+(-) A_{0} Q^{2}
$$

where $A_{0}=\frac{G_{F}}{4 \pi \alpha \sqrt{2}} \sim 1.28 \times 10^{-4}(\mathrm{GeV} / \mathrm{c})^{2}$, and positive (negative) states are for isoscaler (isovector) excitations. This relationship is a simple estimate, and generally good to within a factor of two. It is important to emphasize that this large uncertainty is acceptable in practice so long as the rate fraction for the process is relatively small, as with these resonances.

For discrete, single particle excitation states, the cross-sections are empirical fits from $[134,135]$. Only the lowest-level excited states $(<3.5 \mathrm{MeV})$ contribute, because the form factors for higher level states are suppressed by an order-of-magnitude. No explicit asymmetry correction is made for the single particle states at this time $\left(A_{S P S}=0\right)$, but a large uncertainty of $2.5 \mathrm{ppm}$ is assigned.

The giant dipole resonance is a well studied phenomenon, and the cross-section is estimated by [136]. As an isovector excitation, the asymmetry is negative, and estimated by equation (6.2). To be conservative, an uncertainty of $100 \%$ is attributed to this asymmetry.

Tables 6.7 - 6.9 give the average rate, rate fraction, $Q^{2}$, and asymmetry of each process for the upstream window, downstream window, and solid $4 \%$ downstream target, respectively. These were determined via simulation, and are used to determine the appropriate kinematic correction factors.

The total, kinematically corrected upstream and downstream window asymmetries, 
Upstream Window Summary

\begin{tabular}{|l|c|c|c|c|c|}
\hline Process & Rate $(\mathrm{kHZ} / \mathrm{nA})$ & $\mathrm{f}(\%)$ & $Q^{2}(\mathrm{GeV} / \mathrm{c})^{2}$ & Asymmetry $(\mathrm{ppm})$ & $\mathrm{f}^{*}$ Asym $(\mathrm{ppm})$ \\
\hline Elastic & $4.83 \times 10^{-2}$ & 81 & 0.0200 & 1.786 & 1.441 \\
\hline QE & $6.14 \times 10^{-3}$ & 10 & 0.0235 & -0.900 & 0.092 \\
\hline Inelastic & $3.74 \times 10^{-3}$ & 6.2 & 0.0227 & 2.069 & 0.129 \\
\hline SPS & $1.47 \times 10^{-3}$ & 2.5 & 0.0235 & 0.000 & 0.000 \\
\hline GDR & $2.27 \times 10^{-4}$ & 0.38 & 0.0211 & -2.711 & -0.010 \\
\hline
\end{tabular}

Table 6.7: Average rates, rate fractions, $Q^{2}$, and asymmetries for the upstream target window. The asymmetry determination is discussed in the text.

Downstream Window Summary

\begin{tabular}{|l|c|c|c|c|c|}
\hline Process & Rate $(\mathrm{kHZ} / \mathrm{nA})$ & $\mathrm{f}(\%)$ & $Q^{2}(\mathrm{GeV} / \mathrm{c})^{2}$ & Asymmetry $(\mathrm{ppm})$ & $\mathrm{f}^{*}$ Asym $(\mathrm{ppm})$ \\
\hline Elastic & $3.84 \times 10^{-2}$ & 64 & 0.0237 & 2.113 & 1.357 \\
\hline QE & $7.42 \times 10^{-3}$ & 12 & 0.0285 & -0.900 & -0.112 \\
\hline Inelastic & $3.76 \times 10^{-3}$ & 6.3 & 0.0277 & 2.069 & 0.130 \\
\hline SPS & $1.36 \times 10^{-3}$ & 2.3 & 0.0286 & 0.0000 & 0.000 \\
\hline GDR & $1.94 \times 10^{-4}$ & 0.32 & 0.0247 & -3.180 & -0.010 \\
\hline
\end{tabular}

Table 6.8: Average rates, rate fractions, $Q^{2}$, and asymmetries for the downstream target window. The asymmetry determination is discussed in the text.

$\overline{A^{U S}}$ and $\overline{A^{D S}}$, can be expressed as

$$
\begin{aligned}
& \overline{A^{U S}}=A_{\text {meas }} \times \frac{A_{\text {sim }}^{U S}}{A_{\text {sim }}^{D S-4 \%}}, \text { and } \\
& \overline{A^{D S}}=A_{\text {meas }} \times \frac{A_{\text {sim }}^{D S}}{A_{\text {sim }}^{D S-4 \%}},
\end{aligned}
$$

where the overline reminds us a kinematic correction has been applied to the measured asymmetry, $A_{\text {meas }}$, and $A_{\text {sim }}^{U S}$ and $A_{\text {sim }}^{D S-4 \%}$ are the simulated upstream and $4 \%$ solid target asymmetries, respectively. These include contributions from all scattering processes, each weighted by their relative rate fraction, $f_{i}$. For example, the total weighted solid $4 \%$ target asymmetry is given by

$$
A_{\text {sim }}^{D S-4 \%}=\sum_{i} f_{i}^{D S-4 \%} A_{i}^{D S-4 \%},
$$

where the summation index $i$ runs over each process (elastic, quasielastic, inelastic, single particle excitations, and the giant dipole resonance). $f_{i}^{D S-4 \%}$ and $A_{i}^{D S-4 \%}$ are the process rate fractions and asymmetry contributions from Table 6.9. Similar equations for the upstream and downstream window asymmetries can be written.

The average weighted asymmetry contribution from the windows, $\langle A\rangle_{\text {win }}$, is given 
Downstream 4\% Solid Target Summary

\begin{tabular}{|l|c|c|c|c|c|}
\hline Process & Rate $(\mathrm{kHZ} / \mathrm{nA})$ & $\mathrm{f}(\%)$ & $Q^{2}(\mathrm{GeV} / \mathrm{c})^{2}$ & Asymmetry $(\mathrm{ppm})$ & $\mathrm{f}^{*}$ Asym $(\mathrm{ppm})$ \\
\hline Elastic & $1.49 \times 10^{0}$ & 79 & 0.0232 & 2.0691 & 1.631 \\
\hline QE & $2.14 \times 10^{-1}$ & 11 & 0.0291 & -0.900 & -0.102 \\
\hline Inelastic & $1.09 \times 10^{-1}$ & 5.7 & 0.0277 & 2.0691 & 0.1195 \\
\hline SPS & $6.87 \times 10^{-2}$ & 3.6 & 0.0290 & 0.0000 & 0.0000 \\
\hline GDR & $7.92 \times 10^{-3}$ & 0.42 & 0.0251 & -3.2368 & -0.0136 \\
\hline
\end{tabular}

Table 6.9: Average rates, rate fractions, $Q^{2}$, and asymmetries for the solid $4 \%$ aluminum target. The asymmetry determination is discussed in the text.

as the absolute rate weighted average of each process:

$$
\langle A\rangle_{\text {win }}=\frac{R^{U S} \overline{A^{U S}}+R^{D S} \overline{A^{D S}}}{R_{i}^{U S}+R^{D S}},
$$

where $R^{U S}\left(R^{D S}\right)$ is the total rate of the upstream (downstream) window as determined by simulation. To be conservative, a blanket $5 \%$ uncertainty is given for the knowledge of the absolute elastic scattering rate, and $50 \%$ uncertainties are assigned for the nonelastic rates. The elastic asymmetry uncertainty is assumed $10 \%$, while the other uncertainties are assigned $100 \%$ of their values.

An extra model uncertainty is present for each process, and is determined by varying the rate and asymmetry by its respective uncertainty. The total model uncertainty quoted below is the quadrature sum of these individual process uncertainties. This is likely an overestimate, as it does not take into account correlations between the numerators and denominators of equations (6.3) and (6.4). The final calculated window asymmetry is then given as

$$
\langle A\rangle_{\text {win }}=1.5026 \pm 0.0660 \text { (stat.) } \pm 0.0102 \text { (sys.) } \pm 0.0346 \text { (model) ppm. }
$$

This is a $5 \%$ determination of the aluminum window asymmetry. The leading model uncertainty is driven by the uncertainty of the inelastic contributions, in particular the large inelastic scatters in the radiative tail and the inelastic single particle excited states. 


\section{Chapter 7}

\section{Concluding discussions}

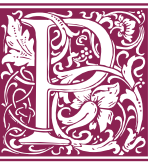

AST Chapters CONCEnTRATed on introducing the Standard Model, $Q_{\text {weak }}$, and several important experiment corrections, namely sub-percent determi-

nation of the electron beam polarization (Chapter 4) and accounting for the large contribution of the target's aluminum walls (Chapters 5 and 6). This final chapter will summarize the findings of those experimental corrections, and then focus on the impact these new results will have in the final $Q_{\text {weak }}$ analysis. It concludes with a summary of suggestions for future work, including a short discussion about extracting the parity-violating asymmetry of pure aluminum from the results obtained in Chapter 6.

\subsection{Summary of findings}

This thesis reports the first sub-percent precision determination of electron beam polarization by the Hall C Møller polarimeter (Chapter 4, Table 4.4). These sub-percent results were limited to $Q_{\text {weak }}$ Run 2. During Run 1, one of the magneto-optical elements (a large quadrupole) developed an intermittent electrical short in one coil. This resulted in a time-dependent magnetic field and asymmetric detector acceptance. To account for this, a magneto-static model was developed and implemented in the Møller Monte Carlo. This model showed good agreement with systematic studies. The final Run 1 polarization was ultimately determined to a precision of $1.10 \%$.

During Run 2, complementary polarization measurements were provided by a new Compton polarimeter, which ran continuously at high current during production running, and also provided sub-percent polarization results. The two polarimeter results agreed on average to within $0.70 \%$, which is within their uncertainties. A crosscalibration between the two was also performed and is the subject of Appendix A. This result is an important ancillary measurement, as it is the first time two indepen- 
dent, sub-percent-precision polarimeters have measured beam polarization in the same experiment hall at Jefferson Lab. Both polarimeter results were in excellent agreement.

The aluminum target window contribution from $Q_{\text {weak }}$ is the largest experimental background, resulting in a $30 \%$ correction to the measured asymmetry. For the published Run 0 results [48], the aluminum dilution was known to only $6 \%$ relative precision, and the aluminum asymmetry was known to $14.4 \%$ of itself. An updated analysis technique was developed for this thesis, with a novel, empirically determined electronic deadtime correction, ultimately leading to determination of the dilution to a relative precision of $2.8 \%$. The total contribution to the measurement of the parityviolating asymmetry uncertainty is then $0.84 \%$; the original Run 0 results contributed roughly $2 \%$.

$Q_{\text {weak }}$ is the first parity-violating experiment to directly measure the parity-violating asymmetry of its target cell; previous experiments relied on simulation to determine the contribution, or placed the target walls outside of the experiment's acceptance. The Run 2 data set resulted in determining the parity-violating asymmetry of aluminum 7075-T651 at $Q^{2}=0.026 \mathrm{GeV}^{2}$ to be $1.6174 \pm 0.0704$ (stat.) \pm 0.011 (sys.) parts-permillion. This is a $4.5 \%$ measurement, and could be used to extract the parity-violating asymmetry of pure aluminum (see Section 7.3). The weighted asymmetry, including kinematic corrections based on the target window locations, is $1.5026 \pm 0.0660$ (stat.) \pm 0.0102 (sys.) \pm 0.0346 (model) ppm. This is a $5 \%$ result.

The work in this thesis thus provides substantial improvement on the previously reported $Q_{\text {weak }}$ results, and has a clear impact on the $Q_{\text {weak }}$ extraction. As shown in Section 7.2 , the updated analysis allows a $5 \%$ analysis of the parity-violating asymmetry from elastic $\bar{e}-p$ scattering, and an approximate $7.5 \%$ determination of $Q_{W}^{p}$.

\subsection{Current Run $2 Q_{\text {weak }}$ Results}

In this section, the current $Q_{\text {weak }}$ results are presented and discussed. It is emphasized that the results presented here include a blinding factor, so true interpretation of these results as a Standard Model test cannot currently be undertaken.

Section 5.1 briefly discussed how $Q_{\text {weak }}$ relates its measured elastic electron-proton asymmetry, $A_{\text {meas }}$, to the true physics asymmetry, $A_{e p}$ :

$$
A_{e p}=R_{t o t} \frac{A_{m s r} / P-\sum_{i} f_{i} A_{i}}{1-\sum_{i} f_{i}}
$$

where $R_{t o t}$ is correction for radiative and acceptance effects, $f_{i}$ and $A_{i}$ are the various background process dilutions and asymmetries respectively, and $P$ is the electron beam 
polarization. Here, $A_{\text {meas }}$ includes several sources of false asymmetries which must be removed:

$$
A_{\text {meas }}=A_{\text {raw }}-A_{\text {beam }}-A_{B B}-A_{T}-A_{L}-A_{\text {blind }},
$$

$A_{\text {raw }}$ is the raw measured asymmetry with no corrections applied, $A_{\text {beam }}$ is the false asymmetry of any helicity-correlated beam properties, $A_{B B}$ is the beamline background asymmetry, $A_{T}$ is the false asymmetry arising from residual transverse polarization, $A_{L}$ is the false asymmetry produced by any main detector non-linearity, and $A_{\text {blind }}$ is the blinding factor. Similar to the aluminum background extraction (Section 6.2), the beamline background is the only background that is explicitly subtracted from the raw asymmetry at the slug level, and thus is not included in the $\sum_{i} f_{i} A_{i}$ factor in the numerator of equation (7.1). It is however included in the denominator dilution summation.

The multiplicative correction encompasses several higher-order processes and kinematic effects:

$$
R_{t o t}=R_{R C} R_{D e t} R_{B i n} R_{Q^{2}}
$$

$R_{R C}$ is a Mo-Tsai type radiative correction [21] deduced from simulations, including bremsstrahlung, from $[137,138]$. $R_{\text {Det }}$ accounts for the measured light variation and nonuniform $Q^{2}$ distribution across the main detector bars. $R_{B i n}$ is an effective kinematics acceptance correction which shifts the asymmetry from $\left\langle A\left(Q^{2}\right)\right\rangle$ to $A\left(\left\langle Q^{2}\right\rangle\right)$. $R_{Q^{2}}$ is the precision in calibrating the central $Q^{2}$ at the scattering vertex. Table 7.1 summarizes the current values and uncertainties of these corrections.

\begin{tabular}{|l|c|c|}
\hline Correction & Value & Uncertainty \\
\hline$R_{R C}$ & 1.0101 & .0007 \\
$R_{\text {Det }}$ & 0.9921 & .0044 \\
$R_{\text {Bin }}$ & 0.9800 & 0.005 \\
$R_{Q^{2}}$ & 1.0000 & .000314 \\
\hline$R_{\text {total }}$ & 0.9820 & 0.0170 \\
\hline
\end{tabular}

Table 7.1: Summary of current Run 2 radiative corrections.

The current estimate of $A_{B B}$ was the subject of [127]. Similar to the aluminum analysis, correlations between the main detector and the background detectors (in particular the upstream luminosity monitors) were used to determine the correction factor. The dilution was measured during systematic studies using tungsten shutters, and these results combined (the correction and dilution) allow extraction of the effective beamline background asymmetry. 
A thorough treatment of the effects of residual transverse beam polarization is provided by [75]; the current results come from an updated, internal analysis. Since transversely polarized electron-proton scattering is a parity-conserving process, any slight residual transverse polarization could have a large effect. This asymmetry was directly measured during dedicated periods with the beam polarization fully transverse. The transverse asymmetry exhibits an azimuthal dependence, which is observed in the main detector rates, and any broken symmetry in the detector chain is observed in the fit. This determines the leakage.

As in the aluminum measurement (Section 6.3.1), the linearity correction, $A_{L}$, accounts for any non-linear detector response. Since the main detector signal is different between running during $\mathrm{LH}_{2}$ and aluminum, the non-linearity is slightly zero. Current estimates give a zero net effect, but to be conservative a large $2 \mathrm{ppb}$ uncertainty is included.

Each $Q_{\text {weak }}$ Run period has a blinding factor. All data within the Run period include this offset, to avoid any sort of systematic bias. The blinding term is randomly generated with uniform probability to be within $\pm 60 \mathrm{ppb}$.

Polarization values came from a combined Møller-Compton analysis, and were applied at the slug level. Six periods of stable beam polarization were determined throughout Run 2, and the average of each was mapped to the slugs for that period. One exception was just after the photocathode heat and re-activation (as seen in Figure 4.19). During this period a best-fit approximation was applied, correlating the measured polarization with slug number. The average polarization was $\langle P\rangle=(88.9 \pm 0.62) \%$, where the uncertainty is the combined Møller-Compton systematic.

The determined asymmetries and dilutions for all the backgrounds are provided in Table 7.2. The most recent value of the measured (blinded) asymmetry is taken from [127]. This does not include the full $Q_{\text {weak }}$ data set; it focuses only on data where beam modulation was functional, and disregards Wien 10. This dataset has a statistical uncertainty of $8.3 \%$, which is a modest increase over the full data set.

There are four main background sources that contribute to the main detector signal and must be removed: the aluminum window contribution, beamline background, the QTOR transport background, and inelastic scattering, primarily through the $N \rightarrow \Delta$ chain. The aluminum window contribution was the subject of this thesis, and in particular Chapters 5 and 6 . The beamline background component was discussed earlier, and also subtracted.

The QTOR transport channel was discussed in regards to the aluminum target 


\begin{tabular}{|l|c|c|}
\hline Contribution & Asymmetry (ppb) & Uncertainty (ppb) \\
\hline$A_{\text {raw }}$ & -159.48 & 8.27 \\
$A_{\text {beam }}$ & -2.0 & 0.82 \\
$A_{B B}$ & 3.84 & 1.59 \\
$A_{T}$ & 0 & 0.5 \\
\hline$A_{L}$ & 0 & 2.0 \\
\hline \hline$A_{\text {meas }}$ & -161.27 & 8.71 \\
\hline
\end{tabular}

Table 7.2: Summary of current Run 2 false asymmetries.

window asymmetry in Section 6.3. Recall that it is generated with scattering off of the collimator slits or shielding wall. The dilution is measured during blocked octant studies, and is consistent with zero. It's asymmetry is simulated, with the assumption that it is identical to its parent process.

The fourth, and final, background contribution comes from inelastic pion production through the $N \rightarrow \Delta$ resonance. As discussed in Section 6.3.2, this process leaks in through the radiative tail due to the large energy acceptance of the QTOR spectrometer. The dilution is determined through simulations, while the asymmetry is measured at the inelastic peak (the magnet setting where the largest rate fraction comes from this inelastic channel). Determinations of the dilution and asymmetry of the $N \rightarrow \Delta$ transition were the subject of [94].

Tables 7.3 and 7.4 summarize the current background contributions in $Q_{\text {weak }}$. Note that the beamline background asymmetry is not provided, as it was removed previously, during the calculation of $A_{\text {meas }}$. Note the total background dilution, $f_{\text {total }}=\sum_{i} f_{i}=$ $3.212 \%$.

\begin{tabular}{|l|c|c|}
\hline Background & Asymmetry $(\mathrm{ppm})$ & Uncertainty $(\mathrm{ppm})$ \\
\hline Target windows & 1.5026 & 0.0752 \\
\hline Beamline & - & - \\
\hline QTOR transport & -0.283 & 0.057 \\
\hline$N \rightarrow \Delta$ resonance & -3.02 & 0.97 \\
\hline
\end{tabular}

Table 7.3: Background asymmetries in $Q_{\text {weak }}$ Run 2.

\begin{tabular}{|l|c|c|}
\hline Background & Dilution & Uncertainty \\
\hline Target windows & 0.02872 & 0.00057 \\
\hline Beamline & 0.00193 & 0.00064 \\
\hline QTOR transport & 0.00127 & 0.00138 \\
\hline$N \rightarrow \Delta$ resonance & 0.0002 & 0.0002 \\
\hline
\end{tabular}

Table 7.4: Background dilutions in $Q_{\text {weak }}$ Run 2. 
Combining the information from Tables 7.1 - 7.3, and substituting into equation (7.1), we find the final (blinded) extracted parity-violating elastic asymmetry to be

$$
A_{P V}=-226.94 \pm 9.44 \text { (stat.) } \pm 6.64 \text { (sys.), }
$$

a $5 \%$ measurement.

Extracting the proton's weak charge is now possible, as discussed in Chapter 2. The fitting procedure, and the world's data, are described, there, and remains essentially the same as [48], except the form-factor parameterization has been updated to use the Arrington-Sick form factors [32] instead of [139]. Figure 7.1 shows the fit and $Q^{2}=0$ extrapolation, and the final weak charge is

$$
Q_{W}^{p}(P V E S)=0.0688 \pm 0.0048(\text { BLINDED }) .
$$

This is in agreement with the Standard Model value of $Q_{W}^{p}=0.0710 \pm 0.0007$, and shows the improvement from the Run 0 analysis discussed in [67] and shown in Figure 2.4. The inset shows the extrapolation to zero- $Q^{2}$. The Standard Model prediction is given by the black-arrow, and the green dotted line is the curve without the $Q_{\text {weak }}$ datum. The yellow-band is the $1 \sigma$ uncertainty band of the fit.

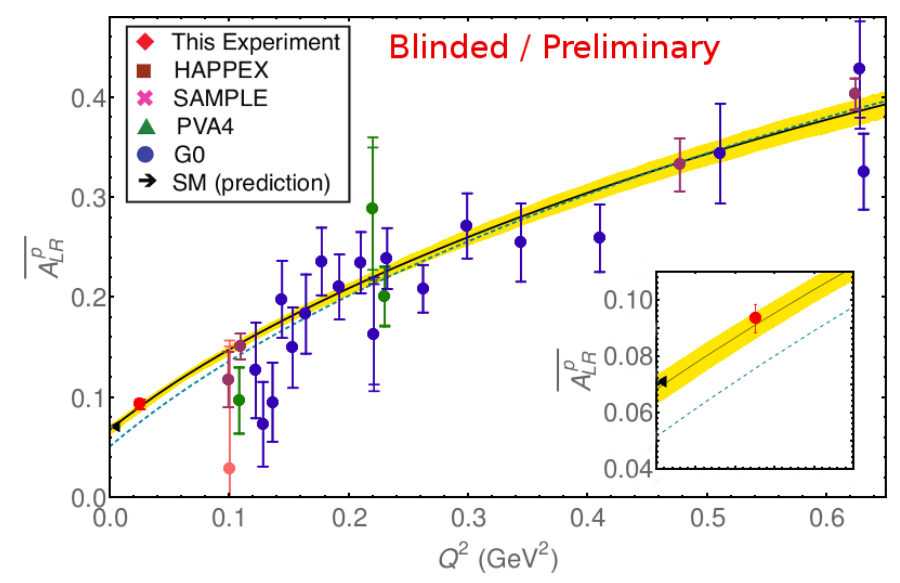

Figure 7.1: Fit to current parity-violating world data set, including the updated blinded $Q_{\text {weak }}$ analysis (red point). The blinding factor is not shown.

Combining these results with the Cesium atomic parity violating experiments enables extraction of the individual quark charges, and determination of the neutron's weak charge as well. Figure 7.2 shows the isoscaler versus isovector linear combinations of quark-vector couplings, $C_{1 u}$ and $C_{1 d}$. The limits on the quark vector charges for the up- and down- quarks are

$$
\begin{aligned}
& C_{1 u}=-0.1860 \text { (BLINDED) } \\
& C_{1 d}=0.3376 \text { (BLINDED) } .
\end{aligned}
$$


From Figure 7.2, these are in agreement with the Standard Model (black dot). The determination of the nucleon weak charges, including the parity-violating results, is

$$
\begin{aligned}
& Q_{W}^{p}=0.06871 \pm 0.0048(\mathrm{BLINDED}) \text { and } \\
& Q_{W}^{n}=-0.9786 \pm 0.0064(\mathrm{BLINDED})
\end{aligned}
$$

The central value could be different due to the blinding factor.

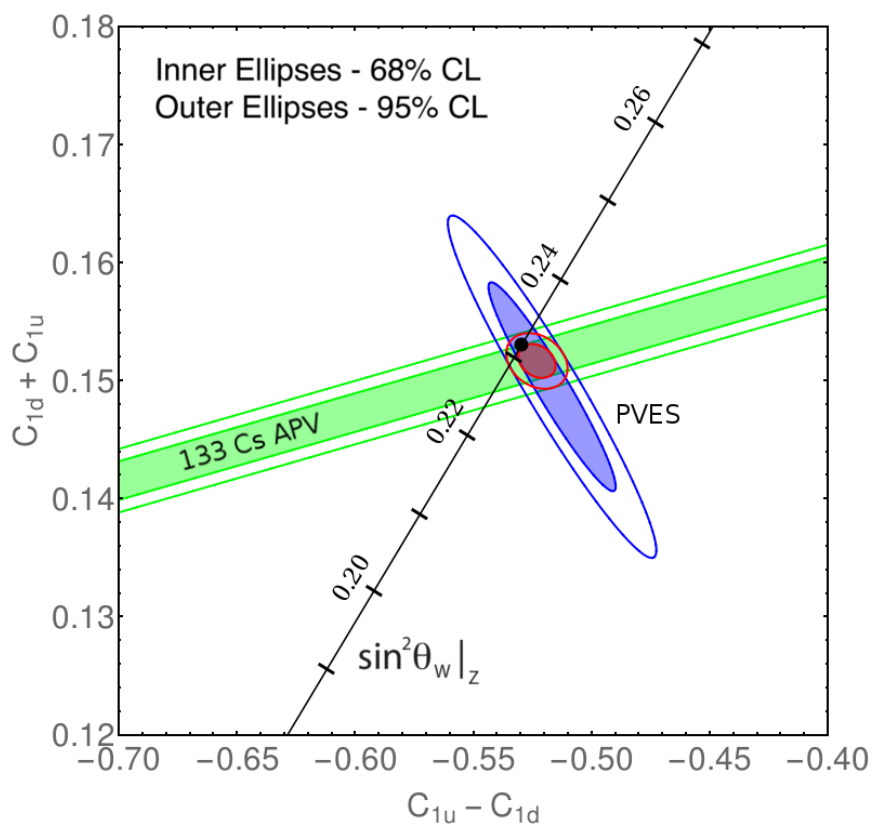

Figure 7.2: Constraints on quark weak-vector couplings, including the improved results from this work. Compare to Figure 2.5.

A model-independent limit on potential new physics can be set from these results using equation (2.35). This estimate shows the particle-mass coupling ratio to be

$$
\frac{\Lambda}{g} \sim 3.56 \mathrm{TeV}(\mathrm{BLINDED}) .
$$

\subsection{Future work}

This thesis resulted in significant improvement in the $Q_{\text {weak }}$ background corrections, in preparation for the upcoming $Q_{\text {weak }}$ data unblinding. Although most future work related to $Q_{\text {weak }}$ should concentrate on other, less-developed analysis tasks, several possible improvements related to this work exist.

The current aluminum asymmetry results only provide an estimate for the quasielastic scattering contribution. A more rigorous model for quasielastic and inelastic asymmetries should be developed, to better bound the uncertainty. Further analysis of the inelastic contribution, which is approximately $5 \%$ of the scattering rate, should also be included. 


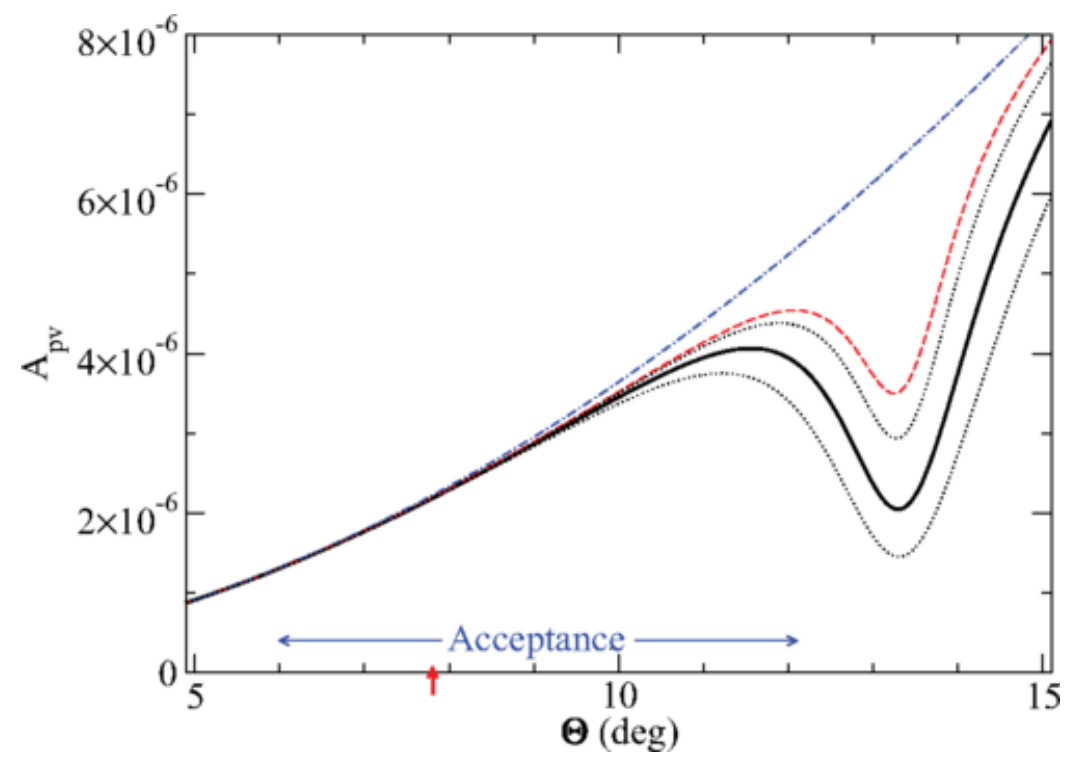

Figure 7.3: The parity-violating asymmetry of elastic scattering between pure aluminum and $1160 \mathrm{MeV}$ electrons vs scattering angle, $\theta$. Reproduced from [47].

The measured asymmetry of aluminum 7075 , reported in Chapter 6 , can be used to extract the parity-violating asymmetry of pure aluminum, which has never been measured. Extracting the asymmetry for pure aluminum would require that nuclear uncertainties to be small, and that the inelastic nuclear excitations and impurities can be effectively removed [47]. For instance, recall that the current estimate for the elastic aluminum parity-violating asymmetry, equation (2.32), assumes dominance of the charged monopole term, and neglects higher-order Coulomb distortion effects. Figure 7.3 shows a recent, improved calculation from [47], using a relativistic mean-field FSU gold- model [140] which includes Coulomb distortions. The blue dot-dashed curve (top line) shows the lowest-order plane wave Born approximation, assuming both the neutron and proton distributions are spherically symmetric. The red dashed line includes the full distorted wave calculations. The solid black line includes higher-order distortions from higher angular-momentum states $(\mathrm{L}=1$ states contribute near the diffraction minima, occurring around $\left.\theta \sim 13^{\circ}\right)$. The $Q_{\text {weak }}$ acceptance is set by the arrows, with the red arrow signaling the average experimental $Q^{2}$. Agreement between all the models is good until $\sim 9^{\circ}$. The disagreement grows, and it is $\sim 6 \%$ at $\theta=11^{\circ}$. However, the cross section drops rapidly at these larger scattering angles, suggesting that once cross-section weighted, the variation in predicted model asymmetry will be manageable.

If extracted, calculations suggest a $4 \%$ measurement of the pure aluminum asymmetry would enable a $2 \%$ determination of the neutron radius in aluminum $\left(R_{n}\right.$ of $\left.{ }^{27} \mathrm{Al}\right)$ [47]. This is because of the large coupling between neutrons and the weak-neutral cur- 
rent. These calculations assume that nuclear structure uncertainties, and the contribution of nuclear inelastic excited states, are well understood. This may prove challenging, but empirically determined neutron radii are currently desired to improve calculations of neutron star properties, and there has been much recent experimental interest in such programs. For perspective, a 2-3\% determination of aluminum's neutron radius would be competitive with determinations from other nuclei. The original PREX experiment measured $R_{n}$ of ${ }^{208} \mathrm{~Pb}$ to $3 \%$ [141]. Proposed follow-up measurements intend to improve this to $1 \%$ [142], while another Jefferson Lab experiment (CREX) aims to measure the $R_{n}$ of ${ }^{48} \mathrm{Ca}$ to $1 \%$ [143].

The aluminum uncertainty has four primary components: the simulation statistics $(0.8 \%)$, the simulation model $(1.6 \%)$, the BCM $(1.5 \%)$, and the electronic deadtime model $(1.5 \%)$. The electronic deadtime model is fixed at $1.5 \%$, but the other three sources may improve somewhat. For example, the simulation statistics can easily be reduced by running further simulations. The BCM contribution could conceivably be reduced somewhat; the current estimate is based on measured differences between the Unser monitor and Faraday Cup at high current $(150 \mu A)$. This 1.5\% discrepancy is suspected to arise from beam transport loss in the injector, and is likely small between $100 \mathrm{nA}$ and $1 \mu \mathrm{A}$. If systematic studies at low-current could be identified, the dominant BCM systematic would then be beam position on the Faraday Cup, which is approximately $1 \%$. This leaves the simulation model uncertainty, which encompasses all differences between the simulated and measured rates. The origin of these discrepancies is presently unknown, but improvements in the inelastic rate calculations could reduce this uncertainty.

The Møller analysis for $Q_{\text {weak }}$ is finalized, however it is strongly suggested that dedicated time be granted in the future for a more precise Møller-Compton cross calibration. The first cross-calibration discussed in Appendix A was quite successful, and demonstrated agreement at approximately the $0.60 \%$ level under identical running conditions. However, it did fall short of setting a definitive $0.50 \%$ limit on any currentdependence of the electron beam polarization. Although no compelling mechanism for such a current-dependence exists, the Hall C Møller analysis is continually burdened with a large high-current extrapolation uncertainty (typically $0.50 \%$ ). The author personally believes this overly conservative, and a few days of dedicated systematic study could easily demonstrate the existence of such effect, or bound it more precisely. 


\title{
Appendix A
}

\section{Møller-Compton Cross Calibration}

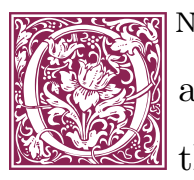

NE OF THE most important Hall C results during the $Q_{\text {weak }}$ experiment was a cross-calibration between the Møller and Compton polarimeters. This was the first direct comparison at the same beam current under indentical beam conditions. This enables a direct investigation and comparison of the systematic differences in the two devices at sub-percent precision. In addition, a precise cross-calibration at low- and high-currents would enable direct observation of any beam current dependence of the electron polarization.

The Møller-Compton cross calibration occurred during Run 2, in May 2012. A full description of the Møller systematics was given in Chapter 4, with a summary table of uncertainties (Table 4.4). Although an introduction to the Compton was given in Section 3.4.2, a full discussion of systematic uncertainties was absent. They will be discussed briefly in Section A.1.
\end{abstract}

\section{A.1 Systematics and method}

One challenge of comparing the Møller and Compton polarimeters is finding a suitable beam current where the Møller could operate without large corrections from target heating and the Compton polarimeter could simultaneously gather adequate statistics in a reasonable time frame. During this study, the Møller measurements were taken at currents over twice the nominal $2 \mu \mathrm{A}$ current, slightly increasing its overall uncertainty.

A beam current scan was performed to determine the highest current at which the Møller could reasonably operate. The results are shown in Figure A.1. The raw polarization (top, blue circles) does not include corrections for target de-polarization due to beam heating. The corrected points (maroon triangles) include a correction based on [109], which was discussed in Section 4.2.2. The dashed line is the best fit 
of the corrected data, and is $86.54 \pm 0.14 \%$ (stat). The fit's reduced $\chi^{2}$ is 0.7 , and the probability of observing a larger value of $\chi^{2}$ by chance is 0.67 , suggesting the fit is appropriate.

Figure A.1, bottom, gives the correction size for both accidents (black triangles) and beam heating (red squares). Although small compared to the beam heating corrections, the accidental corrections are non-negligible even at these modest currents. It was decided that corrections should be no larger than $1 \%$ to reduce additional uncertainty, and the uncertainty of this correction was taken as the conservative value of $30 \%$ of the overall correction size. It was decided to limit the beam current to $4.5 \mu \mathrm{A}$.
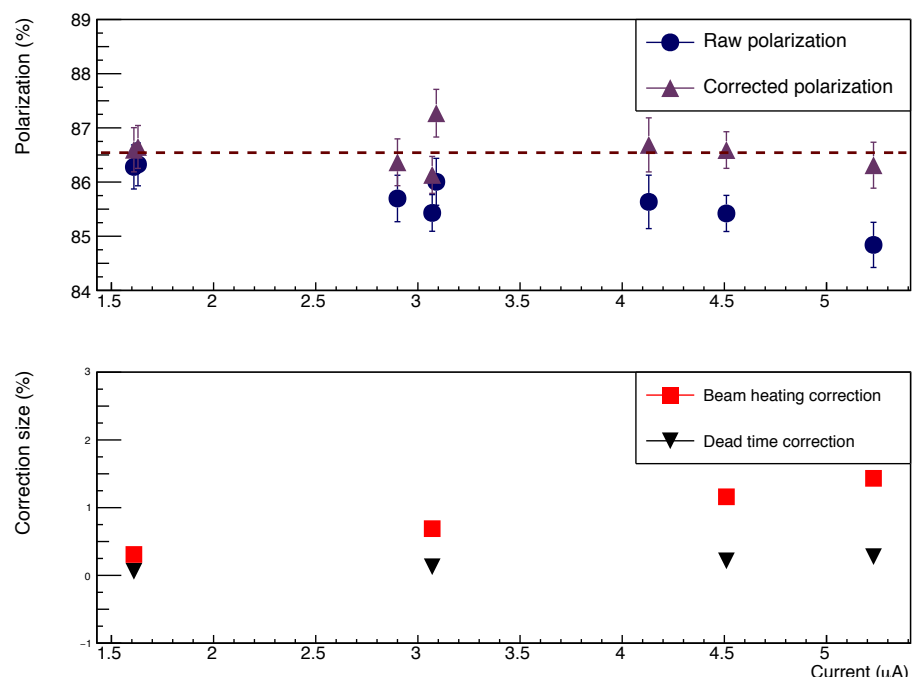

Figure A.1: (Top) Measured polarization from the Møller polarimeter as a function of beam current. (Bottom) Size of corrections for dead time and target de-polarization due to beam heating.

The subsequent Møller uncertainty list for this study is given in Table A.1. This includes the larger target temperature uncertainty and discards the uncertainty due to extrapolation to $180 \mu A$ found in Table 4.4. The total Møller systematic uncertainty is then $0.71 \%$ (at $4.5 \mu A$ ).

For this study, only the Compton electron detector was used. Compton measurements last approximately an hour, during which the measured yield asymmetry is compared to the theoretical asymmetry for each detector strip. The results are fit with two free parameters: the electron beam polarization and the detector strip number corresponding to the maximum electron displacement (also known as the Compton edge). A typical detector asymmetry is shown in Figure A.2. The upper (lower) panel shows the asymmetry and fit for high (low) current; the data are in blue, while the best-fit curve is in red. 


\begin{tabular}{|l|c|c|}
\hline Source & Uncertainty & $\Delta \mathrm{P} / \mathrm{P}(\%)$ \\
\hline Beam position X & $0.2 \mathrm{~mm}$ & 0.14 \\
\hline Beam position Y & $0.2 \mathrm{~mm}$ & 0.28 \\
\hline Beam angle X & $0.5 \mathrm{mrad}$ & 0.10 \\
\hline Beam angle Y & $0.5 \mathrm{mrad}$ & 0.10 \\
\hline Q1 current & $2 \%$ & 0.07 \\
\hline Q3 current & $2 \%$ & 0.05 \\
\hline Q3 position & $1 \mathrm{~mm}$ & 0.10 \\
\hline Multiple scattering & $10 \%$ & 0.01 \\
\hline Levchuk effect & $10 \%$ & 0.33 \\
\hline Fixed collimator positions & $0.5 \mathrm{~mm}$ & 0.03 \\
\hline Target temperature & $30 \%$ & 0.24 \\
\hline B-field direction & 2 degrees & 0.14 \\
\hline B-field strength & $5 \%$ & 0.03 \\
\hline Spin polarization in Fe & - & 0.25 \\
\hline Electronic D.T. & $100 \%$ & 0.045 \\
\hline Solenoid focusing & $100 \%$ & 0.21 \\
\hline Solenoid position $(\mathrm{x}, \mathrm{y})$ & $0.5 \mathrm{~mm}$ & 0.23 \\
\hline Monte Carlo statistics & - & 0.14 \\
\hline Total & & $\mathbf{0 . 7 1}$ \\
\hline
\end{tabular}

Table A.1: Systematic Uncertainties of the Møller Polarimeter during the crosscalibration.

Inefficiencies associated with the Compton DAQ, such as deadtime and triggering inefficiencies, were studied using simulation. Deadtime corrections were negligible at low-current, and $<1 \%$ during high-current running. A full Monte Carlo using GEANT3 [144] was used to study a variety of systematic uncertainties. The full Compton uncertainty budget is shown in Table A.2.

The cross-calibration had three parts: first an 8 hour segment of Compton measurements at high current $(180 \mu A)$, a middle period of measurements with both polarimeters at $4.5 \mu A$ (a Compton measurement sandwiched between two Møller measurements), and a final high-current Compton measurement. In order to keep the electron beam optics identical, the beam was transported through the Compton chicane during all measurements. Even though the beam transport were identical during this comparison, some minor differences did exist between the Møller and Compton measurements. For instance, the Compton laser-table orbit lock could not be used during the Møller measurements. 


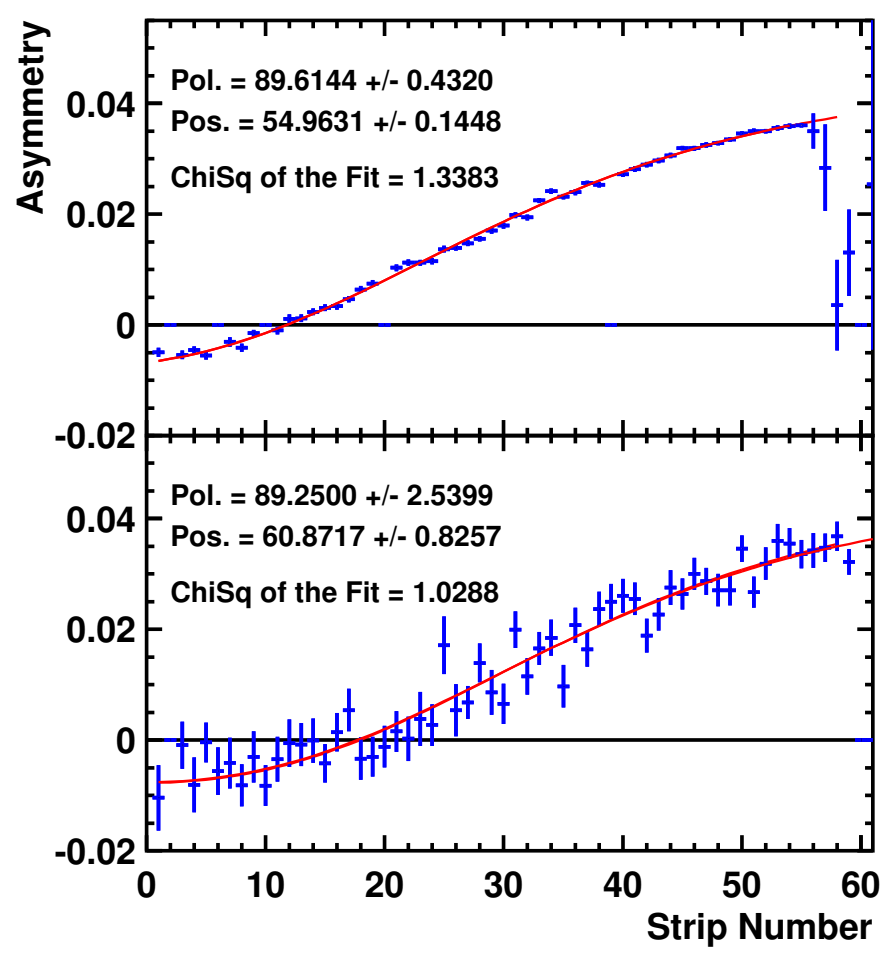

Figure A.2: Measured asymmetry by Compton electron detector strip at high (top) and low (bottom) current. Data are in blue; best-fit curves are in red.

\section{A.2 Data, analysis, and results}

Figure A.3 gives the results of the polarimeter cross-calibration. The measured polarization is given versus Compton run number; each Compton point (high current points are red circles, low-current points are blue) represents an hour long measurement. Each Møller point (black squares) represents the average of all measurements taken at a single beam current. Inner uncertainty bars are statistical, while the outer bars present the statistical and systematic uncertainties combined in quadrature. The bottom plots gives the average results for each measurement. Table A.3 summarizes these results.

Two main results come from these data. First, the average Compton result, 86.99 \pm 0.604 (stat+sys), is in agreement with the Møller result of $85.6 \pm 0.77$ (stat+sys). The difference is $0.45 \%$, well within the uncertainty of both polarimeters and consistent with the Møller-Compton Run 2 results discussed in Section 4.4.

The second result of significant importance is determining if the extracted polarization shows any current dependence. There is no clear, accepted proposed mechanism for this, and all previous studies at JLab have presented no evidence of such an effect up to $60 \mu A[110,113,114]$. However, when applying the Møller measurements to 


\begin{tabular}{|l|c|c|}
\hline Source & Uncertainty & $\Delta \mathrm{P} / \mathrm{P}(\%)$ \\
\hline Laser Polarization & 0.18 & 0.18 \\
Plane to Plane & secondaries & 0.00 \\
magnetic field & $0.0011 \mathrm{~T}$ & 0.13 \\
beam energy & $1 \mathrm{MeV}$ & 0.08 \\
detector z position & $1 \mathrm{~mm}$ & 0.03 \\
inter plane trigger & $1-3 \mathrm{plane}$ & 0.19 \\
trigger clustering & $1-8$ strips & 0.01 \\
detector tilt(w.r.t x) & 1 degree & 0.03 \\
detector tilt(w.r.t y) & 1 degree & 0.02 \\
detector tilt(w.r.t z) & 1 degree & 0.04 \\
detector efficiency & $0.0-1.0$ & 0.1 \\
detector noise & up to $0.2 \%$ of rate & 0.1 \\
fringe field & $100 \%$ & 0.05 \\
radiative corrections & $20 \%$ & 0.05 \\
DAQ inefficiency correction & $40 \%$ & 0.3 \\
DAQ inefficiency pt.-to-pt. & & 0.3 \\
Beam vert. pos. variation & $0.5 \mathrm{mrad}$ & 0.2 \\
helicity correl. beam pos. & $5 \mathrm{~nm}$ & $<0.05$ \\
helicity correl. beam angle & $3 \mathrm{nrad}$ & $<0.05$ \\
spin precession in chicane & $20 \mathrm{mrad}$ & $<0.03$ \\
\hline Total & & $\mathbf{0 . 5 9}$ \\
\hline
\end{tabular}

Table A.2: Systematic Uncertainties of the Compton Polarimeter

\begin{tabular}{|l|c|c|c|c|}
\hline Type & $\begin{array}{c}\text { Current } \\
\mu A\end{array}$ & $\begin{array}{c}\text { Mean } \\
\text { pol. }(\%)\end{array}$ & $\begin{array}{c}\text { Stat. } \\
\text { uncert. }\end{array}$ & $\begin{array}{c}\text { Total } \\
\text { uncert. }\end{array}$ \\
\hline Compton & 180 & 86.92 & 0.15 & 0.61 \\
Møller & 4.5 & 86.54 & 0.14 & 0.72 \\
Compton & 4.5 & 87.44 & 0.71 & 0.92 \\
Compton & 180 & 87.16 & 0.29 & 0.66 \\
\hline
\end{tabular}

Table A.3: Mean polarization measurements during cross-calibration

$Q_{\text {weak }}$ production data, an additional $0.50 \%$ uncertainty was applied to be conservative. This cross-calibration could potentially limit this uncertainty, if the polarization results agree at high and low currents.

To estimate any current dependence, the best-fit line of the measured polarization versus current was calculated. The Compton points included only statistical and point-to-point uncertainties; the common scale/normalization Compton systematic was included as an additional uncertainty on the (independent) Møller point. In this way, the fit included this uncertainty only once, and all data were treated as equivalent, aside from statistics. The result, shown in Figure A.4, shows the best-fit slope is $(-3.068 \pm 330.4) \times 10^{-5} \% / \mu A$. The polarization uncertainty from this extrapolation, 


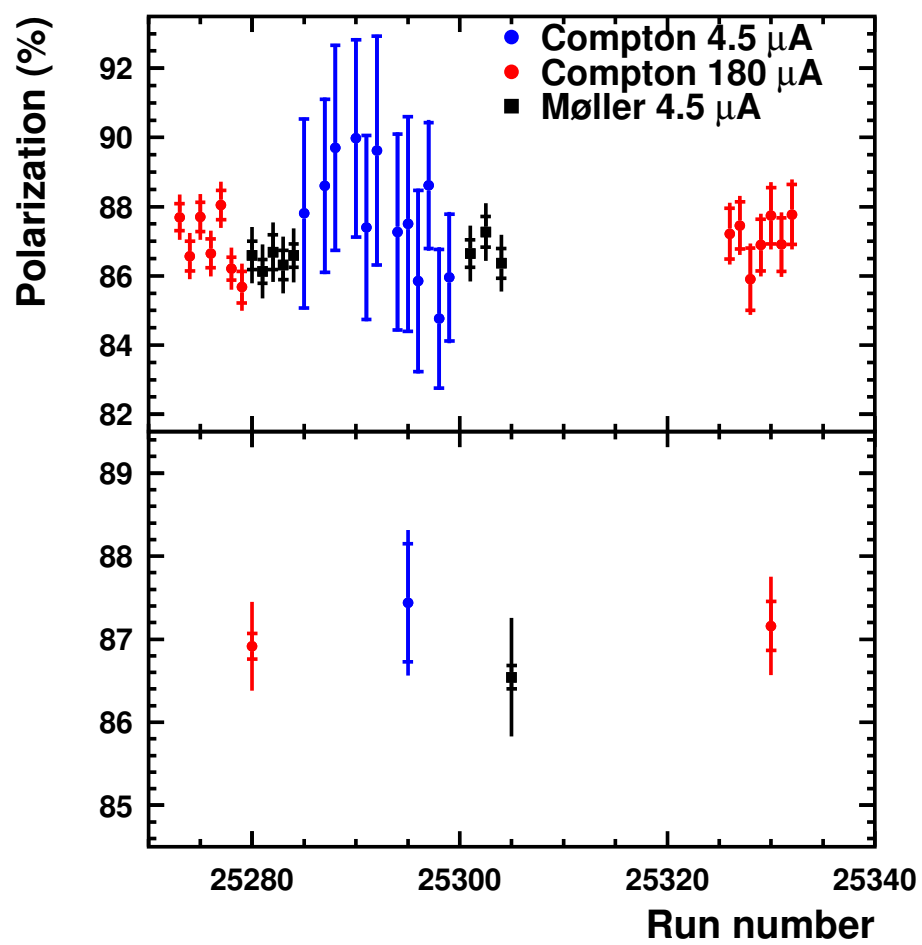

Figure A.3: (Top) Measured polarization versus Compton run number. (Bottom) Average polarization for each measurement period in the study.

$(\delta P)_{I}$, is therefore

$$
\begin{aligned}
(\delta I)_{P} & =\Delta I \cdot\left(\frac{\partial P}{\partial I}\right)=(175.5 \mu \mathrm{A}) \cdot(0.0033 \% / \mu A) \\
& =0.57 \%
\end{aligned}
$$

This estimate is slightly larger than the $0.50 \%$ currently being included in the full $Q_{\text {weak }}$ analysis. In the future it would be of great interest to complete a similar study, but with an even longer low-current Compton data period to further reduce statistics. 


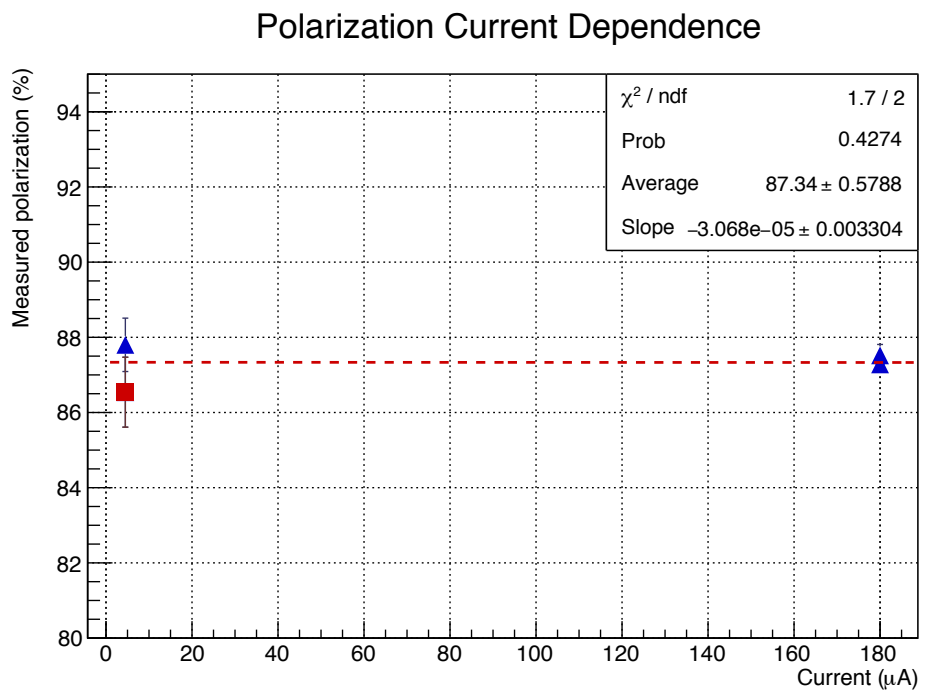

Figure A.4: Measured polarization at high and low current. Compton data are blue triangles, while the red square is the Møller datum. The red dotted line is the best-fit of all data. 


\section{Appendix B}

\section{Alternate Rate Determinations}

EASURING MODERATE SCATTERING rates and cross sections is typically considered trivial, as the technology and methods are well established. In Chap-

ter 5 the difficulties of making absolute scattering rate measurements in parity experiments became clear: often the detectors and their electronics are designed with different needs in mind than counting experiments. In Section 5.2 it was mentioned that three distinct analysis methods were devised to extract absolute rates in $Q_{\text {weak }}$ :

- A Scaler-based analysis

- An Event-mode analysis

- A combined scaler/event-mode hybrid analysis

Chapter 5 focused on the hybrid analysis, which combined rate extraction from the scaler banks but used elements of the event-mode analysis to determine the main detector deadtime. In this appendix the original scaler based analysis and event-mode analysis will be discussed.

\section{B.1 Scaler based main detector deadtime determination}

Extracting rates from scalers, including subtraction of accidental background, was discussed fully in Section 5.2.1 and 5.2.1. The problem with a purely scaler analysis is effectively determining the main detector electronic deadtime, which is known to be variable due to long analog pulse length coupled with a short 40 ns discriminator output width. Instead, we must parameterize the electronic deadtime constant, $\tau$ from rates scans on solid targets. From equation (5.8)

$$
L T_{\text {elec }}=e^{R \tau} \approx 1-R_{t o t} \cdot \tau,
$$


where $R_{t o t}$ is the total rate measured by that bar. The goal will be to extract $\tau$ from the solid target running, using the information that an increase in beam current should result in a proportional increase in scattering events. Sadly, a proper rate scan wasn't performed on the solid carbon target; only two different currents were measured. A three-point rate scan was conducted on the main $Q_{\text {weak }}$ target, evacuated of $\mathrm{LH}_{2}$, which is used as an example.

Figure B.1 shows the three measured rates on the evacuated $Q_{\text {weak }} \mathrm{LH}_{2}$ target. The left plot shows the measured scattering rates as a function of beam current. These rates include small corrections for accidentals. The right plot shows the current-normalized yield, which should be constant. These data are fit to a first-order polynomial, which is extrapolated to zero current to determine the true yield, where no deadtime would be present. In this example, the true yield would be $127.6 \mathrm{kHz} / \mu A$. The relative rate between the measured and true rates is calculated for each point, and this is fit to a decaying exponential, as suggested by equation (5.8) (Figure B.2). The second fit parameter (the slope-like term in the expansion) gives the decay constant, $\tau$. In this example, $\tau \sim 205$ ns. Finally, correcting the measured rates by this $\tau$ shows the yield is now current independent as expected (Figure B.2, right).

This $\tau$ extraction was performed for all eight octants on three separate targets: evacuated hydrogen target, the solid carbon dummy target, and a thin aluminum optics target (Optics \#3). The results are shown in Table B.1. Note the Run 2 Carbon and Optics targets show agreement within 20-30 ns, while the evacuated $\mathrm{LH}_{2}$ target shows large discrepancies. This is likely because the rates are much larger on the solid targets (see Tables 5.2 and 5.3, for example), providing a more accurate determination of $\tau$ from a longer "level arm." It is also possible, perhaps even likely, that the $\tau \mathrm{s}$ do depend somewhat on the target, because each target provides a different scattering profile on the main detector face, leading to different light distribution along the bar. Recall from Section 5.2.1 that the main detector dead time is a complicated function of several main detector parameters, namely of pulse size and threshold. The extracted $\tau \mathrm{s}$ are closely related, and will likewise exhibit similar octant-dependent behavior.

The $\tau$ s extracted from the Carbon target match fairly well the measured double pulse resolutions from Section 5.2.1 for both $Q_{\text {weak }}$ Runs. The Run $2 \tau$ s from Carbon also agree relatively well with the Optics-3 target. As briefly mentioned earlier, the evacuated target $\tau \mathrm{s}$ are over a rather limited rate range and therefore of limited applicability, and disagree with $\tau \mathrm{s}$ from the higher rate targets. The source of the disagreement is undetermined. 

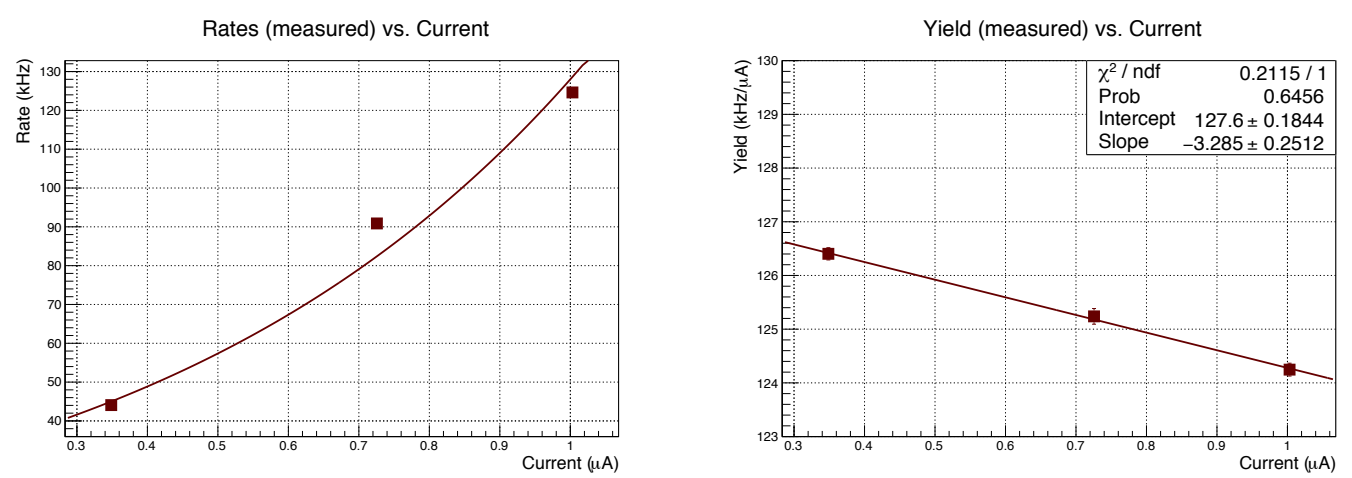

Figure B.1: (Left) Measured rates as a function of current from an empty target cell. An exponential fit guides the eye. (Right) Current-normalized rates show an aberrant current dependence. The fit intercept approximates the true rate, where there would be no dead time.
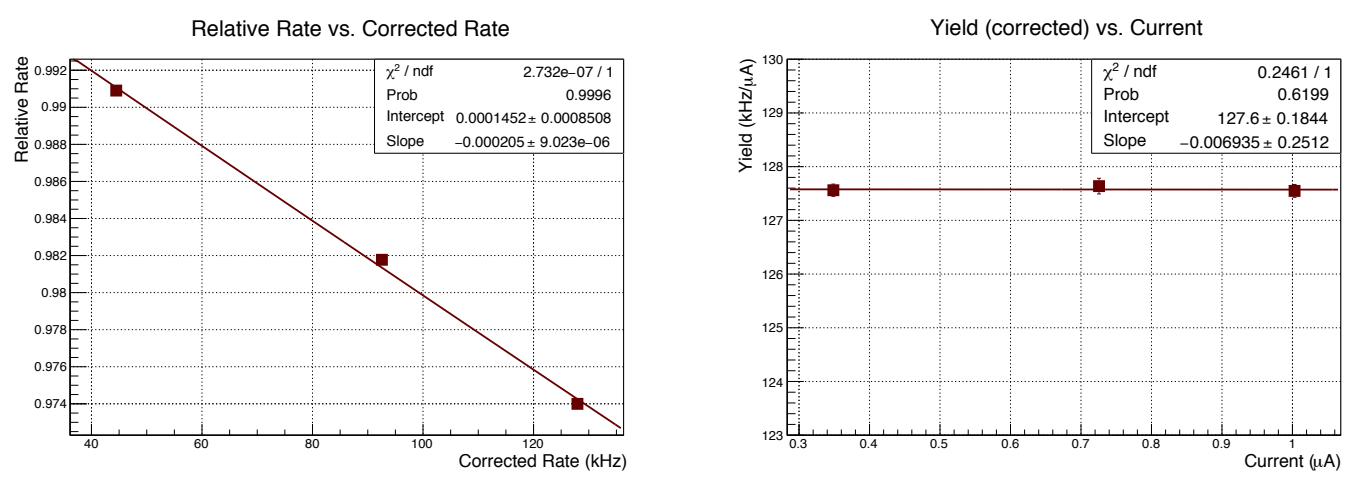

Figure B.2: (Left) Measured rate over true rate extracted from Figure B.1. The fit is a decaying exponential; the slope term is $\tau$, in $\mathrm{kHz}^{-1}$, or ms. (Right) The measured yield is current-independent after correcting by $\tau$.

\begin{tabular}{|l|c|c|c|c|}
\hline \multirow{2}{*}{ Octant } & $\begin{array}{c}\text { Run 1 } \\
\text { Carbon }\end{array}$ & \multicolumn{3}{|c|}{ Run 2 } \\
Evacuated target & Carbon & Optics-3 \\
\hline 1 & 343 & 205 & 152 & 155 \\
\hline 2 & 629 & 230 & 252 & 209 \\
\hline 3 & 414 & 256 & 179 & 175 \\
\hline 4 & 623 & 246 & 166 & 151 \\
\hline 5 & 367 & 246 & 180 & 156 \\
\hline 6 & 375 & 250 & 189 & 158 \\
\hline 7 & 341 & 225 & 185 & 165 \\
\hline 8 & 364 & 180 & 165 & 153 \\
\hline
\end{tabular}

Table B.1: Deadtime constants $(\tau \mathrm{s})$ extracted from the main detector scaler responses in both $Q_{\text {weak }}$ Runs. The $\tau$ s are in ns. 
The deadtime constants were extracted over half a dozen data sets in Run 2, once in January 2012 and with several runs in May 2012. They were also extracted using data from a hydrogen gas target. All of these are roughly consistent with the data shown in Table B.1, suggesting the $\tau$ s are stable.

Scripts to determine these scaler $\tau \mathrm{s}$ were developed and are currently part of the $Q_{\text {weak }}$ analysis package, QwAnalysis.

\section{B.2 Event-mode technique}

The event-mode analysis was developed to further suppress any accidental background included in the scalers by requiring a coincidence with the trigger scintillators. Additionally, the main detectors have a small sensitivity to neutral particles, via second order processes due to the photoelectric effect (photons eject an atomic electron in the silicon lattice) or via pair-production in the silicon nuclear field. These electrons (and positrons, in the case of pair production) can also produce Cherenkov light. These backgrounds, including other neutral processes, are present in a scaler only analysis but are removed when requiring a coincidence between the main detectors and trigger scintillators. Although conceptually clean and powerful for this reason, the event-mode analysis was plagued with many subtle problems. It really highlights how different parity experiments, and their associated data acquisition systems, are from traditional cross-section measurements.

Due to the different DAQ setup and use of the trigger scintillator as a triggering device, calculating the current-normalized rate, $Y$, in event-mode requires additional knowledge. This rate can be calculated as

$$
Y=\frac{P \cdot(R-A)}{(L T)_{\mathrm{TS}} \cdot(L T)_{\mathrm{MD}} \cdot(L T)_{\mathrm{DAQ}} \cdot(L T)_{\mathrm{trig}}} \frac{1}{t \cdot\langle I\rangle},
$$

where $R$ is the raw measured scattering rate, $A$ is the accidental contribution to be removed, and $(L T)_{\mathrm{TS}},(L T)_{\mathrm{MD}}$, and $(L T)_{\mathrm{DAQ}},(L T)_{\text {trig }}$ are the livetimes of the trigger scintillator, main detector, DAQ, and trigger respectively. The event-mode DAQ is prescaled by a factor $P$, meaning only $1 / P$ events is read-out by the DAQ. ${ }^{1} t$ is the time of the run segment analysis. The Sections B.2.1 and B.2.2 cover the differences in how rates, accidentals, and deadtime, and $t$ are computed in event mode. The average current, $\langle I\rangle$, and its uncertainty, is determined by the low-current BCM calibration as discussed in Section 5.2.1.

\footnotetext{
${ }^{1}$ Every event is in principle counted, although only a fraction of events have all of the electronics signals recorded.
} 


\section{B.2.1 Rates in event-mode}

All event-mode analysis used the trigger scintillator as the event trigger, instead of the $10 \mathrm{~Hz}$ MPS clock. The main component of the event-mode analysis were the F1TDCs, which enabled observing multi-hit structures (see Section 5.2.1). The F1TDCs had an open ring buffer, and recorded up to 7 hits for each individual PMT. When a trigger occurred, the gate was opened $300 \mathrm{~ns}$ early, for a total $2000 \mathrm{~ns}$ window. With such a large opening gate, properly forming coincidences between the detectors was problematic since real hits in one detector might improperly be matched with early hits (accidentals) in the other detector. When matching two detector signal, we are attempting to marry them.

The trigger scintillator used a hardware meantime unit, which married the trigger scintillator PMT signals automatically, greatly simplifying analysis. The main detector lacked such a unit, and the PMT signals had to be married in software. Once a proper main detector mean time was constructed, the marriage procedure was then repeated to connect the real main detector events with trigger scintillator ones.

The marriage algorithm used was a modified Gale and Shapley solution to the Stable Marriage Problem [145], where the goal is to optimally match two sets (a bride set and a groom set) according to a list of preferences. The algorithm finds a stable solution, defined as no possible pairs existing where both members would be happier than they currently are. The original problem required both groom and bride sets containing the same number of elements; in the case of the two detectors, the number of hits seen by the trigger scintillator was often larger than the number of main detector hits. This was from the larger areal size and faster electronics chain for the trigger scintillator compared to the main detectors. To account for this, the typically smaller main detector set was increased in size with a bunch of unphysical hit times, and any potential events without a potential partner within 30 ns were married to these unphysical entries.

A toy example is given in Figure B.3, where signals from the main detector and trigger scintillator are married. This example has three recorded hits in the trigger scintillator; events 1 and 3 are unambiguous, true elastic events, while event 2 is outside of the $Q_{\text {weak }}$ acceptance (perhaps a double scatter or some neutral background). The right side of Figure B.3 is the detector's digitized signal. Without proper marriage between the detectors, the spurious TS hit combines with the MD signal from event 3, leading to an improperly determined mean-time and ultimately, incorrect counting. When events are time-ordered properly this problem becomes clear: the second trigger 
scintillator hit should be discarded, leaving only two real hits for this event.

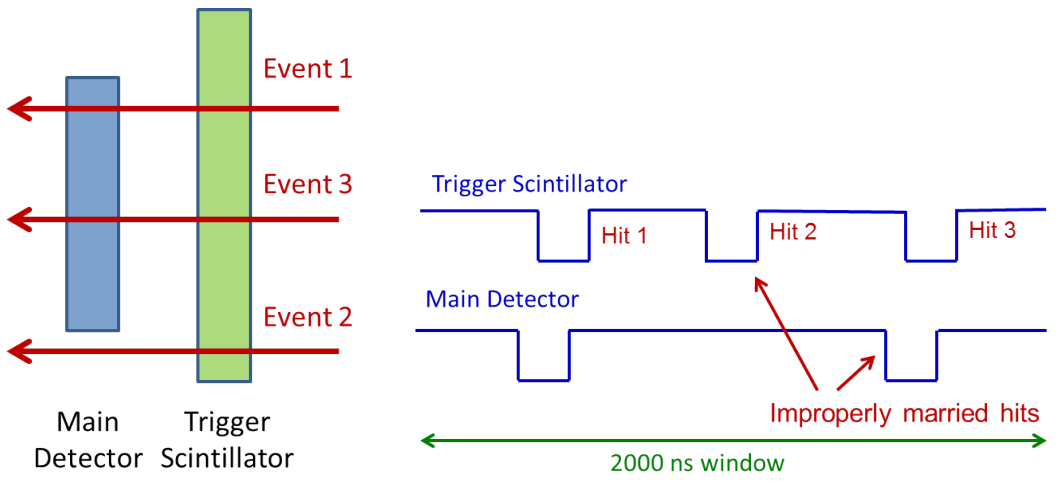

Figure B.3: Example marriage of trigger scintillator and main detector signals for 2 real events and 1 accidental. (Left) The physical process. (Right) The electronic signal.

The meantime of each detector's photomultiplier tubes was calculated using this marriage algorithm. Example meantime spectra for the trigger scintillator and main detector are shown in Figure B.4. The trigger is easily identifiable, being several orders of magnitude above background. This is suggestive that any accidental corrections will be on the order of a percent. The trigger scintillator has a wide $150 \mathrm{~ns}$ gap before the trigger, corresponding to the F1TDC discriminator width. The main detector shows different behavior, presumably due to the pulse-height dependent deadtime (see Section 5.2.1). This also explains why the main detector peak displays a larger width: it takes a non-trivial amount of time for photon collection to finish.
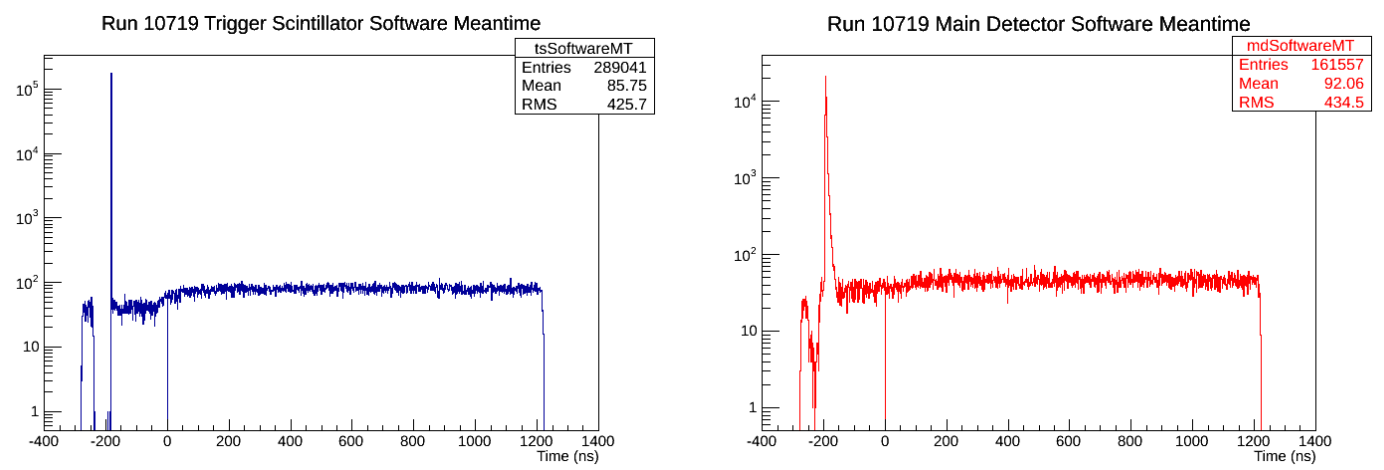

Figure B.4: Trigger scintillator (left) and main detector software meantimes (right).

Once the event meantimes were determined, a coincidence was formed between the main detector and trigger scintillator. Only triggering events were considered, i.e., only those in the trigger scintillator peak. The resultant spectra is shown in Figure B.5. The raw, unmarried main detector spectra is shown in red, while the true events, those that are seen both by the main detector and trigger scintillator, are shown in blue. The 
background signal is further reduced an extra half-order of magnitude in this example, and is roughly $0.1 \%$. Depending on the rate and target, this additional background suppression may be larger.

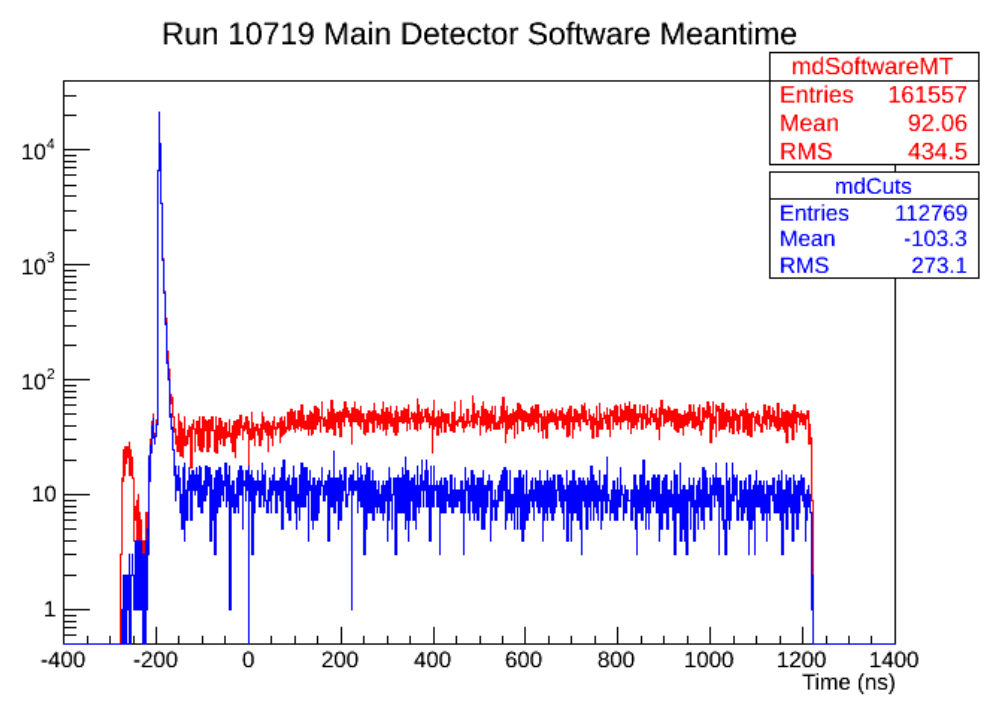

Figure B.5: Overlay of the raw main detector spectra (red), with the spectra of events seen also by the trigger scintillator (blue).

Determining the current-normalized scattering rate is fairly straightforward at this point. The number of events in the combined TS.MD peak are counted, where the peak window is about 60 ns wide. The average number of background events in this 60 ns window width are calculated from the rest of the distribution. Fortunately, it is Poissonian (i.e., flat), and easy to determine.

\section{B.2.2 Detector deadtimes}

The main detector electronic deadtime is discussed in Section 5.2.1. Ideally, since the main detector deadtime is larger than the trigger scintillator deadtime, this would be the limiting effect. However, there are further complications caused by the main detector's variable deadtimes.

Unlike the main detector, the trigger scintillator has narrow pulses and a large discriminator width $(\Delta T=140 \mathrm{~ns}$ [146]), so for any trigger scintillator related electronic deadtime, equation (5.8) applies. The average main detector double pulse resolution is $\sim 150 \mathrm{~ns}$, incredibly close to the trigger scintillator discriminator width. Therefore, for small amplitude events which allow the main detector to re-fire below $<140 \mathrm{~ns}$, the trigger scintillator is the limiting electronic deadtime.

To make the correction, we need to determine the fraction of events that meet this small pulse criteria. Instead of using the main detector triggered double pulse 
resolutions determined from Figures 5.9 and 5.10, the double pulse resolutions are computed for each individual data segment. The fraction of events with small pulse sizes are found (i.e., smaller double pulse resolution than $140 \mathrm{~ns}$ ), and applied as the $(T S)_{\mathrm{TS}}$. In practice, the fraction of events meeting this criteria is $2 \%$ at most. Although individualized for each run, this information is only available for the two triggering octants.

\section{B.2.3 Prescaling, DAQ deadtime, and time determination}

One unique aspect of the event-mode analysis is the use of prescaling. Due to the very small scattering angles and therefore large cross sections, the scattering rates can be quite high even at low-currents. In particular, the thick solid carbon targets can cause rates of $>1 \mathrm{MHz} /$ detector. Since the maximum DAQ rate was $\approx 6 \mathrm{kHz}$, only a subset of events were read-out and recorded by the DAQto minimize computer deadtime. Essentially, the DAQ uses a scaler to count the number of events, but the electronics are read-out only every $P^{\text {th }}$ event. This scaler, part of the trigger supervisor, had a maximum event rate of $3 \mathrm{MHz}$ [147].

Appropriate prescaling was a crucial part of the systematic design; prescales were typically set to keep the trigger read-out rate at approximately $1 \mathrm{kHz}$. Generally, the prescale was assumed exact, and any effects of improper prescaling were accounted for in the DAQ deadtime correction, $(L T)_{\mathrm{DAQ}}$. The DAQ deadtime was determined by on-board scaler clocks, which recorded the number of triggers received and the number of triggers read-out. The DAQ deadtime was simply the ratio of the two. For properly prescaled runs this correction was below $0.5 \% .^{2}$

There were some periods in Run 2 where the prescale was accidentally set to 0 (every event was an attempted read-out), inducing high DAQ deadtime. A study was conducted late in Run 2 to investigate effects of improper prescaling, which enabled a double check of the nominal DAQ deadtime correction. The results of this study are shown in Figure B.6, and show minimal effects. The calculated rate increases about $4 \%$ at low-prescale, but this corresponds perfectly with a corresponding beam current increase of the same size, so is a real shift.

The complete lack of agreement between the scaler analysis and event-mode analysis in Figure B.6 is disturbing. Although the scalers may have small neutral contributions, these are bounded by direct measurements and simulation to be sub-percent level. The disagreement is at the $10 \%$ level, and this is a common feature among all the data

\footnotetext{
${ }^{2} \mathrm{~A}$ similar $200 \mathrm{kHz}$ clock also enabled time determination of the run segment being analyzed.
} 
Event-mode Prescale Study (Run 2)

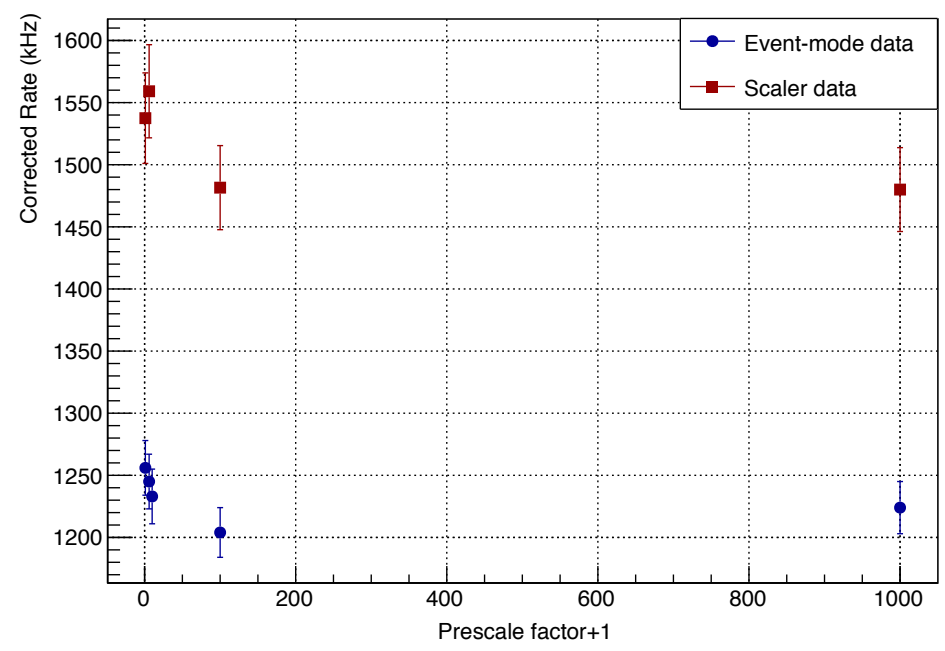

Figure B.6: Determined scattering rate as a function of prescale factor. The abscissa is $P+1$ for ease of plotting.

sets (evacuated target, full target, solid targets). The cause of this discrepancy is still unknown, but discussed further in Section B.3.

There is one additional DAQ-based correction to be made. The problem comes in how the trigger scintillator electronics were configured (see Figure 5.3). Although the limiting detector deadtime is the $\sim 150 \mathrm{~ns}$ discriminator deadtime, the meantime unit has an output width of $60 \mathrm{~ns}$, and essentially goes straight through to the fan-in-fan-out (FIFO) and trigger supervisor. The FIFO output stays true so long as the input stays true, so one trigger scintillator package can essentially veto the other trigger scintillator package, if the second package sends a trigger within $60 \mathrm{~ns}$ of the first.

This is a subtle correction. Whereas the $(L T)_{\mathrm{TS}}$ correction affects the rate in that detector independently of the other, this trigger deadtime, $(L T)_{\text {trig }}$, can affect the overall number of recorded triggers. Assuming both detector packages record identical rates, this would correspond to an additional deadtime of $30 \mathrm{~ns}$ to the overall rate. This is applied to both the trigger scintillator and main detector. This correction has been included in the prescale study in Figure B.6, and does not account for the scaler to event-mode discrepancy.

\section{B.3 Data analysis}

The true test of any low-current tracking analysis is comparing the calculated currentnormalized rates of a solid target at different beam currents. The ratio of high- and low-current measurements should be unity, within some uncertainty. In Section 5.2.2 
Carbon Reference Target Normalization (Run 2)

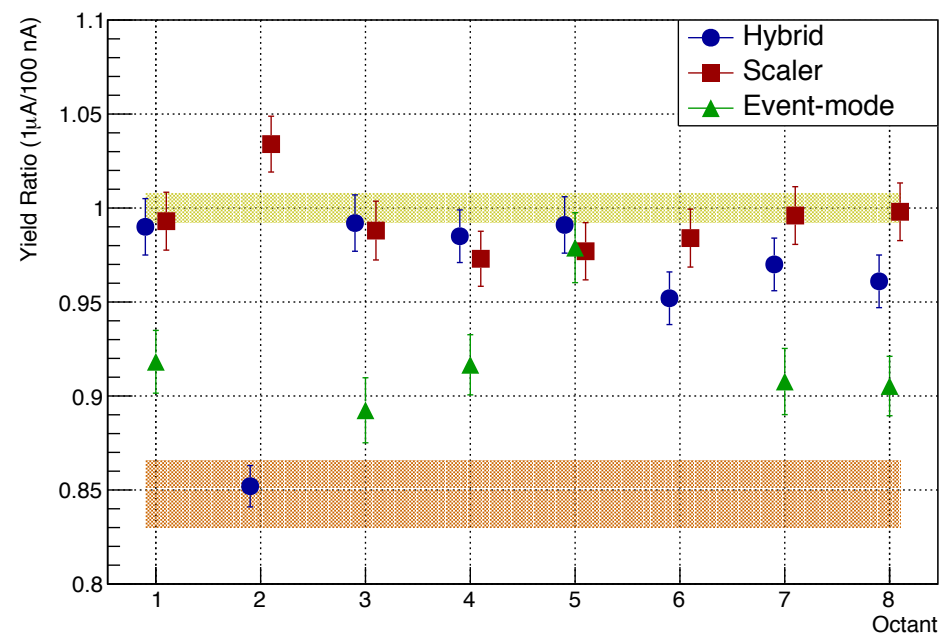

Figure B.7: Ratio of scattering rate on a Carbon target at high beam current to low beam current.

it was clear that this ratio was indeed unity for the Carbon target, albeit with a large model uncertainty from the main detector deadtime correction.

Figure B.7 shows the ratio of the scattering rate off a 1.6\% radiation-length Carbon target of high current $(1 \mu A)$ to low current $(0.1 \mu A)$. The blue circles are from the hybrid analysis (scaler results with the proper main detector double pulse resolution as determined in Section 5.2.1), red squares are scaler results using the rate-scan $\tau$ 's as discussed earlier in Section B.1, and green triangles are the event-mode data. The event-mode data lack octants 2 and 6 due to DAQ problems during data taking. The yellow band is a $1.5 \% \mathrm{BCM}$ uncertainty; the main detector deadtime model uncertainty of $4.3 \%$ is given by the orange box.

Although the scaler and hybrid results are both close to the expected value of 1.00, the event-mode data appears to have an additional rate-dependence of unknown origin. These data include all the relevant corrections, although it should be noted that many of the corrections are small (trigger scintillator deadtime, DAQ deadtime, prescale effects, uncertainty in the time determination), and even if they were increased an order of magnitude could not account for this discrepancy. After an exhaustive search, no cause or explanation could be found. Instead, it was realized that combining the scaler analysis with the event-mode determination of double pulse resolutions would lead to an effective analysis. 


\section{Bibliography}

Some cited documents refer to internal $Q_{\text {weak }}$ technical notes. For assistance accessing them, please contact me directly at magee@jlab.org.

[1] E. Rutherford, Philosophical Magazine 21, 669 (1911).

[2] E. Rutherford, Nuclear constitution of atoms, in Proceedings of the Royal Society, Series A (Containing Papers of a Mathematical and Physical Character (19051934)), volume 97, pages 374-400, 1920.

[3] S. L. Glashow, J. Iliopoulos, and L. Maiani, Phys. Rev. D 2, 1285 (1970).

[4] S. Weinberg, Phys. Rev. Lett. 19, 1264 (1967).

[5] A. Salam, Weak and electromagnetic interactions, in Elementary particle theory, edited by N. Svartholm, pages 367-377, Almquist \& Wiksell, 1968.

[6] G. Aad et al., Phys. Rev. Lett. 114, 191803 (2015).

[7] R. Aaij et al., Phys. Rev. Lett. 115, 072001 (2015).

[8] E. Noether, Nachrichten von der Königl. Gesellschaft der Wissenschaften und der Georg-Augusts-Universitt zu Göttingen, Mathematisch-Physikalische , 235 (1918).

[9] C. Burgess and G. Moore, The Standard Model: A Primer, Cambridge books online, Cambridge University Press, 2007.

[10] T. D. Lee and C. N. Yang, Phys. Rev. 104, 254 (1956).

[11] C. S. Wu et al., Phys. Rev. 105, 1413 (1957).

[12] F. Englert and R. Brout, Phys. Rev. Lett. 13, 321 (1964).

[13] P. Higgs, Physics Letters 12, 132 (1964). 
[14] P. W. Higgs, Phys. Rev. Lett. 13, 508 (1964).

[15] F. Halzen and A. Martin, Quarks and Leptons: an Introductory Course in Modern Particle Physics, Wiley, 1984.

[16] D. Griffiths, Introduction to Elementary Particles, Physics textbook, Wiley, 2008.

[17] M. Peskin and D. Schroeder, An Introduction To Quantum Field Theory, Frontiers in physics, Westview Press, 1995.

[18] H. Georgi, Weak interactions and modern particle theory, Dover books on physics, Dover, New York, NY, 2009.

[19] T. P. Cheng and L. F. Li, Gauge Theory of Elementary Particle Physics, Oxford University Press, 1984.

[20] K. A. Olive et al., Chin. Phys. C38, 090001 (2014).

[21] L. W. MO and Y. S. TSAI, Rev. Mod. Phys. 41, 205 (1969).

[22] J. Erler and M. J. Ramsey-Musolf, Phys. Rev. D 72, 073003 (2005).

[23] A. Czarnecki and W. J. Marciano, International Journal of Modern Physics A 15, 2365 (2000).

[24] B. Rislow, Low Energy Tests of the Standard Model, PhD thesis, The College of William and Mary, 2013.

[25] J. Erler, A. Kurylov, and M. Ramsey-Musolf, Physical Review D 68, 016006 (2003).

[26] P. G. Blunden, W. Melnitchouk, and A. W. Thomas, Phys. Rev. Lett. 107, 081801 (2011).

[27] N. Hall et al., Physics Letters B 753, 221 (2016).

[28] K. Chetyrkin, J. Khn, and M. Steinhauser, Physics Letters B 351, 331 (1995).

[29] S. Galster et al., Nuclear Physics B 32, 221 (1971).

[30] M. Kohl, Nuclear Physics A 805, 361c (2008).

[31] X. Zhan et al., Physics Letters B 705, 59 (2011).

[32] J. Arrington and I. Sick, Phys. Rev. C 76, 035201 (2007). 
[33] J. C. Bernauer et al., Phys. Rev. Lett. 105, 242001 (2010).

[34] J. Arrington, Phys. Rev. Lett. 107, 119101 (2011).

[35] M. Musolf et al., Physics Reports 239, 1 (1994).

[36] Z. Ahmed et al., Phys. Rev. Lett. 108, 102001 (2012).

[37] D. Armstrong and R. McKeown, Annual Review of Nuclear and Particle Science 62, 337 (2012).

[38] H. Budd, A. Bodek, and J. Arrington, Modeling quasi-elastic form factors for electron and neutrino scattering, 2003.

[39] S.-L. Zhu et al., Physical Review D 62, 033008 (2000).

[40] R. D. Young et al., Phys. Rev. Lett. 97, 102002 (2006).

[41] T. Stovall, D. Vinciguerra, and M. Bernheim, Nuclear Physics A 91, 513 (1967).

[42] K. Myers, The First Determination of the Proton's Weak Charge Through ParityViolating Asymmetry Measurements in Elastic $e+p$ and $e+A l$ Scattering, $\mathrm{PhD}$ thesis, The George Washington University, 2012.

[43] R. Lombard and G. Bishop, Nuclear Physics A 101, 601 (1967).

[44] G. Li, M. Yearian, and I. Sick, Physical Review C 9, 1861 (1974).

[45] R. Young et al., Physical Review Letters 99, 122003 (2007).

[46] T. Donnelly, Progress in Particle and Nuclear Physics 24, 179 (1990).

[47] C. J. Horowitz, Phys. Rev. C 89, 045503 (2014).

[48] D. Androic et al., Phys. Rev. Lett. 111, 141803 (2013).

[49] A. Acha et al., Phys. Rev. Lett. 98, 032301 (2007).

[50] T. M. Ito et al., Phys. Rev. Lett. 92, 102003 (2004).

[51] F. E. Maas et al., Phys. Rev. Lett. 94, 152001 (2005).

[52] D. S. Armstrong et al., Phys. Rev. Lett. 95, 092001 (2005).

[53] D. Androić et al., Phys. Rev. Lett. 104, 012001 (2010).

[54] N. L. Hall et al., Phys. Rev. D 88, 013011 (2013). 
[55] M. Gorchtein, C. J. Horowitz, and M. J. Ramsey-Musolf, Phys. Rev. C 84, 015502 (2011).

[56] C. S. Wood et al., Science 275, 1759 (1997).

[57] S. C. Bennett and C. E. Wieman, Phys. Rev. Lett. 82, 2484 (1999).

[58] V. A. Dzuba et al., Phys. Rev. Lett. 109, 203003 (2012).

[59] P. L. Anthony et al., Phys. Rev. Lett. 95, 081601 (2005).

[60] G. P. Zeller et al., Phys. Rev. Lett. 88, 091802 (2002).

[61] K. Kumar et al., Annual Review of Nuclear and Particle Science 63, 237 (2013).

[62] I. Cloët, W. Bentz, and A. Thomas, Physical Review Letters 102, 252301 (2009).

[63] K. Cheung, Physics Letters B 517, 167 (2001).

[64] P. Langacker, Rev. Mod. Phys. 81, 1199 (2009).

[65] H. Davoudiasl, H.-S. Lee, and W. J. Marciano, Phys. Rev. D 92, 055005 (2015).

[66] M. Ramsey-Musolf and S. Su, Physics Reports 456, 1 (2008).

[67] D. Androic et al., Nucl. Instr. and Methods A 781, 105 (2015).

[68] M. Poelker et al., AIP Conference Proceedings 980, 73 (2008).

[69] C. K. Sinclair et al., Physical Review Special Topics - Accelerators and Beams 10, $1(2007)$.

[70] C. Leemann, D. Douglas, and G. Krafft, Annual Review of Nuclear and Particle Science 51, 413 (2001).

[71] D. Androic et al., Nucl. Instr. and Methods A 646, 59 (2011).

[72] J. Grames et al., Two Wien Filter Spin Flipper, in Proceedings of 2011 Particle Accelerator Conference, pages 862-864, 2011.

[73] C. Sinclair, Accelerator Setups Which Provide Longitudinal Beam Polarization To More Than One Experimental Hall Simulataneously, Technical report, Thomas Jefferson National Accelerator Facility, 1996.

[74] A. Hofler et al., Proceedings of International Conference on Particle Accelerators , 3 (1993). 
[75] B. P. Waidyawansa, A 3\% Measurement of the Beam Normal Single Spin Asymmetry in Forward Angle Elastic Electron-Proton Scattering using the Qweak Setup, PhD thesis, Ohio University, 2013.

[76] C. Yan et al., Nucl. Instr. Methods A 365, 261 (1995).

[77] D. Mack, private communication, 2015.

[78] A. Subedi, Determination of the Weak Charge of the Proton through Parity Violating Asymmetry Measurement in the Elastic e+p Scattering, PhD thesis, Mississippi State University, 2014.

[79] G. Niculescu, Resonant Cavities used as Beam Current Monitors, Technical report, Thomas Jefferson National Accelerator Facility, 2015.

[80] J. Bergoz, Nuclear Physics A 525, 595 (1991).

[81] C. Yan, R. Carlini, and D. Neuffer, Proceedings of International Conference on Particle Accelerators, 2136 (1993).

[82] J. R. Hoskins, Determination of the Proton's Weak Charge via Parity Violating Electron Scattering, PhD thesis, The College of William and Mary, 2015.

[83] D. Jones, Measuring the Weak Charge of the Proton via Elastic Electron-Proton Scattering, PhD thesis, University of Virginia, 2015.

[84] M. Hauger et al., Nuclear Instruments and Methods in Physics Rpesearch Section A: Accelerators, Spectrometers, Detectors and Associated Equipment 462, 382 (2001).

[85] L. de Bever et al., Nuclear Instruments and Methods in Physics Research Section A: Accelerators, Spectrometers, Detectors and Associated Equipment 400, 379 (1997).

[86] Polarized Sources, Targets, and Polarimetry, Sub-percent Møller Polarimetery in Jefferson Lab Hall C, Proceedings of Science, 2013.

[87] A. Narayan, Determination of Electron Beam Polarization using Electron Detector in Compton Polarimeter with Less than 1\% Statistical and Systematic Uncertainty, $\mathrm{PhD}$ thesis, Mississippi State University, 2015. 
[88] J. Cornejo, Compton Scattering Polarimetry for the Determination of the Proton's Weak Charge Through Measurements of the Parity-Violating Asymmetry of ${ }^{1} H\left(e, e^{\prime}\right) p$, PhD thesis, The College of William and Mary, 2015.

[89] A. I. H. Committee, ASM Handbook, Volume 02 - Properties and Selection: Nonferrous Alloys and Special-Purpose Materials, 1990.

[90] G. Smith, The Qweak Target Performance, in Il Nuovo Cimento, pages 159-163, 2012.

[91] G. Smith, Solid Target Thickness Measurements, Qweak Electronic Logbook, 2013, https://qweak.jlab.org/elog/Target/21.

[92] Alpha scientific electronics, http://www.alphascientific.com/.

[93] P. Wang, A Measurement Of The Protons Weak Charge Using An Integration Cerenkov Detector System, PhD thesis, University of Manitoba, 2011.

[94] J. Leacock, Measuring the Weak Charge of the Proton and the Hadronic Parity Violation of the $N \rightarrow \Delta$ Transition, PhD thesis, Virginia Tech, 2012.

[95] J. Leckey, The First Direct Measurement of the Weak Charge of the Proton, PhD thesis, College of William and Mary, 2012.

[96] R. Zielinski, Testing and Analysis of $Q_{\text {weak }}$ 's Multiplexing Electronics System, Honors thesis, College of William and Mary, 2010.

[97] D. Ramsey, Current Mode Electronics for the Q-weak Experiment (qweak doc\#552-v1), Technical report, University of Manitoba, 2006.

[98] K. L. Brown, Adv.Part.Phys. 1, 1 (1968 (revised 1982)).

[99] D. C. Carey, K. Brown, and F. Rothacker, (1998).

[100] H. K. and R. Holsinger, SUPERFISH - A Computer Program for Evaluation of RF Cavities with Cylindrical Symmetry, in Particle Accelerators 7, pages 213-222, 1976.

[101] J. A. Magee, The Q-weak Run 2 Møller Polarimetry Analysis, Technical report, The College of William and Mary, 2014.

[102] L. G. Levchuk, Nuclear Instruments and Methods in Physics Research Section A: Accelerators, Spectrometers, Detectors and Associated Equipment 345, 496 (1994). 
[103] M. Loppacher, Møller Polarimetry for CEBAF Hall C, Master's thesis, University of Basel, 1996.

[104] R. Reck and D. Fry, Physical Review 184, 492 (1969).

[105] R. Pauthenet, Journal of Applied Physics 53, 2029 (1982).

[106] D. Gaskell et al., Møller measurements and beam polarization for $Q_{\text {weak }}^{p}$ wien-0, Technical report, Jefferson Lab, 2012.

[107] J. C. D. Graham, Journal of Applied Physics 53, 2032 (1982).

[108] H. Danan, A. Herr, and A. J. P. Meyer, Journal of Applied Physics 39, 669 (1968).

[109] J. Crangle and G. Goodman, Proceedings of the Royal Society of London A , 477 (1971).

[110] D. Gaskell, Calculations of target heating for high current Møller polarimetry, Technical report, Jefferson Lab Hall C Document Database \#771, 2004.

[111] R. Powell, C. Ho, and P. Liley, Thermal Conductivity of Selected Materials, Number v. 1 in NSRDS-NBS, U.S. Dept. of Commerce, National Bureau of Standards; for sale by the Superintendent of Documents, U.S. Govt. Print. Off., 1966.

[112] R. Beminiwattha, Moller Solenoid Scan using $1 \mu \mathrm{m}$ Iron Foil Target, Qweak Electronic Logbook, 2010, https://qweak.jlab.org/elog/Moller/5.

[113] D. Gaskell, D. Meekins, and C. Yan, European Physical Journal A 32, 561 (2007).

[114] M. Poelker et al., Phys. Rev. ST Accel. Beams 10, 053502 (2007).

[115] J. Magee, Effect of IHWP 1 and 2 in Run 2, Qweak Electronic Logbook, 2013, https://qweak.jlab.org/elog/Moller/116.

[116] G. Braun et al., F1: An Eight channel time to digital converter chip for high rate experiments, in Fifth Workshop on electronics for LHC experiments, Snowmass, CO, USA, 20-24 Sep 1999: Proceedings, 1999.

[117] J. H. Lee, How multihits of f1tdc change our naive software meantime histograms, Qweak Electronic Logbook, 2012, https://qweak.jlab.org/elog/Detector/54.

[118] J. H. Lee, F1TDC and its Multihit Histograms, Qweak Document 1604-v2, 2012, https://qweak.jlab.org/doc-private/ShowDocument?docid=1604. 
[119] W. Leo, Techniques for Nuclear and Particle Physics Experiments: A How-To Approach, Springer, 1994.

[120] S. Covrig, private communication, 2015.

[121] S. Agostinelli et al., Nuclear Instruments and Methods in Physics Research Section A: Accelerators, Spectrometers, Detectors and Associated Equipment 506, 250 (2003).

[122] R. S. Beminiwattha, A Measurement of the Weak Charge of the Proton through Parity Violating Electron Scattering using the Qweak Apparatus: A 21\% Result, Phd thesis, Ohio State University, 2013.

[123] J. A. Magee, Run 2 4\%-ds aluminum data quality + cuts, Qweak Electronic Logbook, 2013.

[124] K. E. Myers, Run2 pass5b aluminum: good for gui changes 1/29/2015, Qweak Electronic Logbook, 2015.

[125] K. E. Myers, Run2 dataquality/aluminum summary, Qweak Electronic Logbook, 2015.

[126] M. Pitt, private communication, 2015.

[127] E. Kargiantoulakis, A Precision Test of the Standard Model vis Parity-Violating Electron Scattering in the $Q_{\text {weak }}$ Experiment, $\mathrm{PhD}$ thesis, University of Virginia, 2015.

[128] W. Duvall, November 2015 nonlinearity results, Qweak Electronic Logbook, 2015.

[129] M. E. Christy and P. E. Bosted, Phys. Rev. C 81, 055213 (2010).

[130] P. E. Bosted and V. Mamyan, Empirical fit to electron-nucleus scattering, 2012.

[131] T. Donnelly et al., Nuclear Physics A 541, 525 (1992).

[132] E. Hadjimichael, G. I. Poulis, and T. W. Donnelly, Phys. Rev. C 45, 2666 (1992).

[133] R. Silwal and R. Michaels, Asymmetry Estimates for Inelastic States and Isotope Contamination, Technical report, Thomas Jefferson National Accelerator Facility, 2013.

[134] R. S. Hicks et al., Phys. Rev. C 21, 2177 (1980). 
[135] P. J. Ryan et al., Phys. Rev. C 27, 2515 (1983).

[136] J. Goldemberg et al., Nuclear Physics 43, 242 (1963).

[137] K. A. Aniol et al., Phys. Rev. Lett. 82, 1096 (1999).

[138] K. A. Aniol et al., Phys. Rev. C 69, 065501 (2004).

[139] J. J. Kelly, Phys. Rev. C 70, 068202 (2004).

[140] B. G. Todd-Rutel and J. Piekarewicz, Phys. Rev. Lett. 95, 122501 (2005).

[141] S. Abrahamyan et al., Phys. Rev. Lett. 108, 112502 (2012).

[142] R. Michaels, Electroweak measurements of neutron densities in prex and crex at jlab, usa, 2015.

[143] J. Mammei et al., CREX: Parity-Violating Measurement of the Weak Charge Distribution of ${ }^{48} \mathrm{Ca}$ to $0.02 \mathrm{fm}$ Accuracy, 2013.

[144] R. Brun et al., Simulation program for particle physics experiments, GEANT: user guide and reference manual, CERN, Geneva, 1978.

[145] D. Gale and L. S. Shapley, The American Mathematical Monthly 69, 9 (1962).

[146] J. Magee and K. Myers, Ts discriminator deadtime measurement, Qweak Electronic Logbook, 2011, https://qweak.jlab.org/elog/Tracking/64.

[147] E. Jastrzembski, Trigger supervisor v.2, Technical report, Thomas Jefferson National Accelerator Facility, 2009. 